\title{
ANALYSIS AND MITIGATION OF THE CUBESAT DYNAMIC ENVIRONMENT
}

\author{
A Thesis \\ presented to \\ the Faculty of California Polytechnic State University, \\ San Luis Obispo
}

\author{
In Partial Fulfillment \\ of the Requirements for the Degree \\ Master of Science
}

by

Steve Furger

May 2013 
(C) 2013

Steve Furger

ALL RIGHTS RESERVED

Page ii 
COMMITTEE MEMBERSHIP

TITLE:

AUTHOR:

DATE SUBMITTED:

COMMITTEE CHAIR:

COMMITTEE MEMBER:

COMMITTEE MEMBER:

COMMITTEE MEMBER:
Analysis and Mitigation of the CubeSat Dynamic Environment

Steve Furger

May 2013

Dr. Jordi Puig-Suari, Professor Aerospace Engineering Department

Dr. Faysal Kolkailah, Professor Aerospace Engineering Department

Dr. Eltahry Elghandour, Professor

Civil/Aerospace Engineering Department

Justin Carnahan

CubeSat Program 


\begin{abstract}
Analysis and Mitigation of the CubeSat Dynamic Environment Steve Furger
\end{abstract}

A vibration model was developed for CubeSats inside the Poly-Picosatellite Orbital Deployer (P-POD). CubeSats are fixed in the $\mathrm{Z}$ axis of deployers, and therefore resonate with deployer peaks. CubeSats generally start fixed in the $\mathrm{X}$ and $\mathrm{Y}$ axes, and then settle into an isolated position. CubeSats do not resonate with deployers after settling into an isolated position. Experimental data shows that the P-POD amplifies vibration loads when CubeSats are fixed in the deployer, and vibration loads are reduced when the CubeSats settle into an isolated position. A concept for a future deployer was proposed that isolates CubeSats from the deployer at the rail interface using viscoelastic foam sandwiched in the deployer rails. By creating an isolator frequency far below the deployer resonant frequency, CubeSats loads are not amplified at the deployer's resonant peak. Feasibility tests show that CubeSat vibration loads can be reduced to $50 \%$ of the vibration input in certain cases. Testing also shows that it is much easier to define vibration loads for isolated CubeSats than CubeSats in the current P-POD.

Keywords: CubeSat, P-POD, Vibration, Dynamics, Isolation 


\section{ACKNOWLEDGMENTS}

I would like to thank my mother and father, who have instilled in me the need to pursue higher education from day one. They have always been there, motivating me to be my best.

I owe a large debt of gratitude to Dr. P, Roland, and the personnel of the CubeSat program past and present. Because of them, I was able to build space grade jack-in-theboxes to help fund my education instead of working at Albertson's or something of that sort.

I would like to thank everyone in the CubeSat lab for providing me a fun place between classes and during all-nighters. A special thanks to PolySat for dealing with CubeSat.

I thank Trigger for being the best dog in the world and lowering my stress throughout college.

Finally, I would like to thank America. 


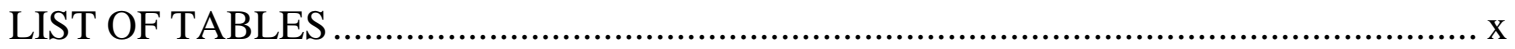

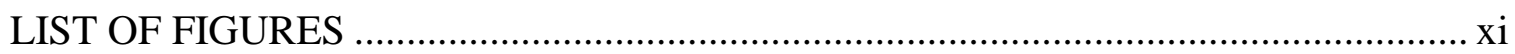

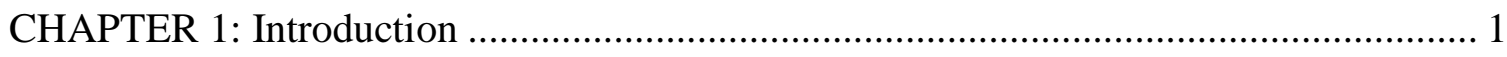

The CubeSat Program ……………………………….................................. 1

Thesis Motivation and Goal......................................................................... 4

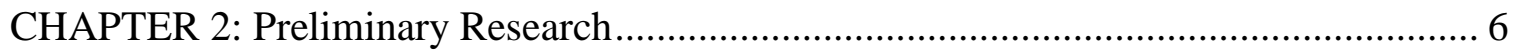

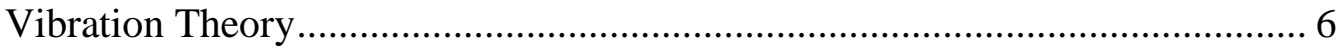

Single Degree of Freedom Spring Mass Damper Model................................. 6

Transmissibility and Steady State Frequency Response to Base Excitation .. 8

Frequency Response Function ................................................................. 8

Regions of Amplification and Isolation................................................. 10

Multiple Degrees of Freedom .................................................................... 11

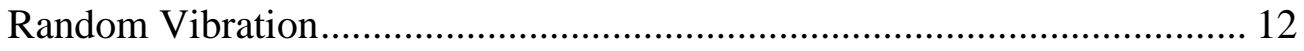

Vibration Mitigation Techniques ...................................................................... 14

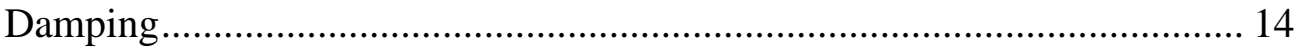

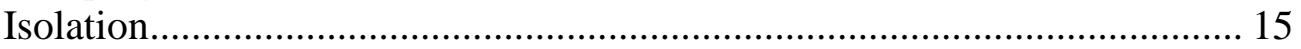

Comparison of Isolation and Damping ........................................................ 16

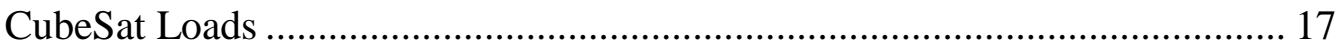

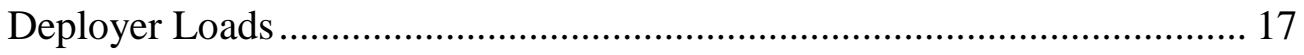

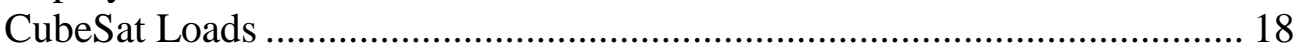

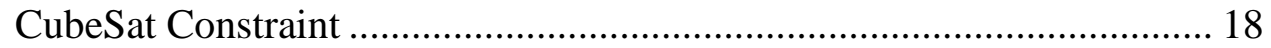

Deployer's Effect on CubeSat Vibration Loads ……………………........ 20

CHAPTER 3: Vibration Testing ............................................................................... 22

Vibration Equipment and Test Setup............................................................ 22

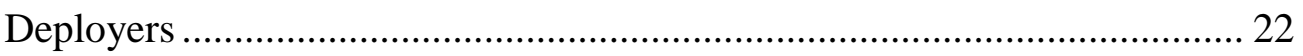

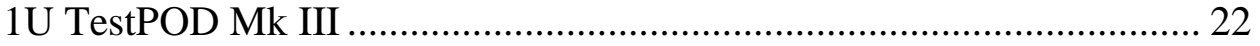

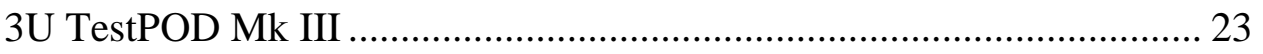

P-POD Mk. III Rev C ................................................................... 24

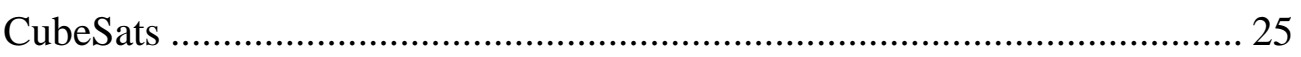


CubeSat Mass Models......................................................................... 25

Test CubeSat ................................................................................ 27

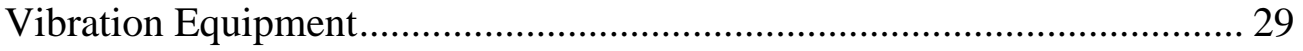

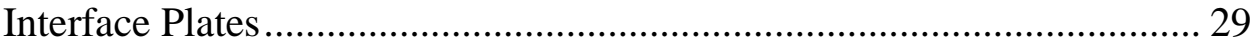

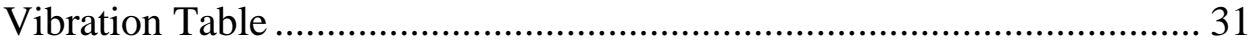

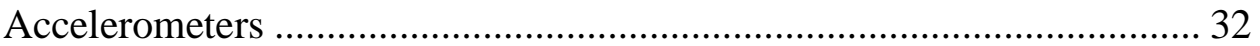

Data Acquisition System.................................................................... 34

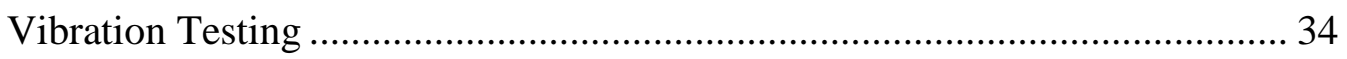

Vibration Test Profiles ............................................................................ 34

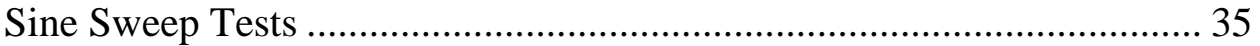

Random Vibration Tests ........................................................................ 36

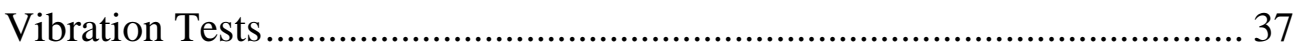

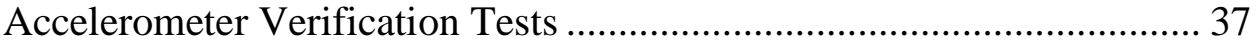

CubeSat and Deployer Testing ...................................................... 41

CHAPTER 4: Modeling the CubeSat-Deployer System ............................................ 43

The CubeSat Constraint in the Deployer ...................................................... 43

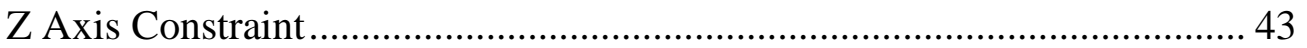

$\mathrm{X}$ and $\mathrm{Y}$ Axes Constraints................................................................. 47

Effect of Canister Constrained CubeSat .................................................. 47

Effect of Friction from Spring Plungers on X and Y Axes Constraint..... 49

CubeSat Slip Load in X and Y Axes ............................................... 51

$\mathrm{X}$ and $\mathrm{Y}$ Axes Constraint with Standard Integration ............................. 53

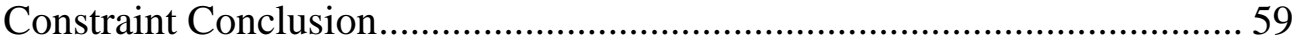

Developing a Vibration Model for the CubeSat-Deployer System .................. 60

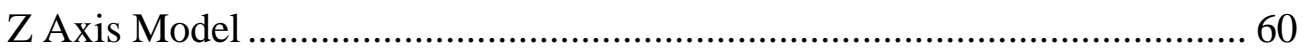

Load Path Model............................................................................ 60

Transfer Function (FRF) Model...................................................... 61

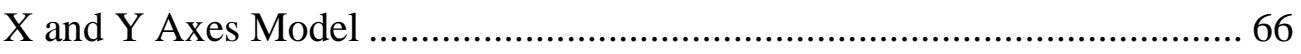

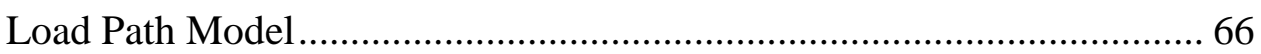

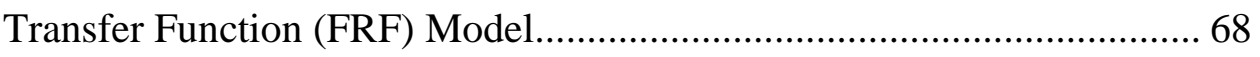

Modeling CubeSat Response in X and Y Axes .................................. 73

Vibration Model Conclusion................................................................... 77

Effect of Deployer Design on CubeSat Loads ............................................ 80

Deployer Design's Effect in the Z Axis................................................. 80 
Deployer Design's Effect in X and Y Axes........................................ 82

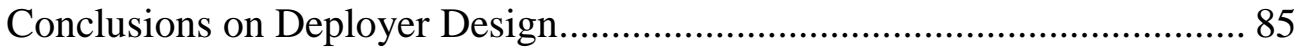

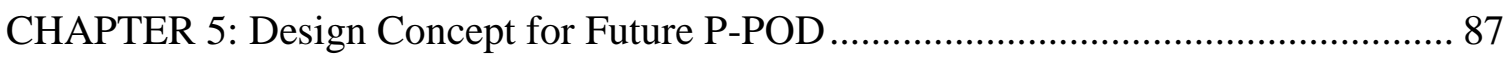

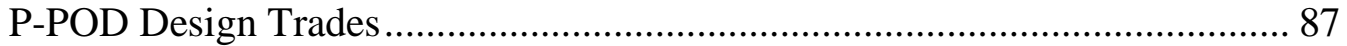

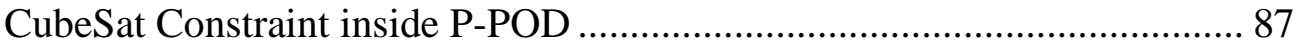

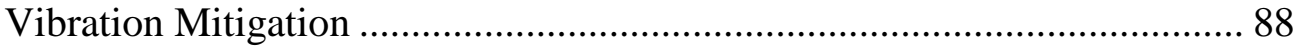

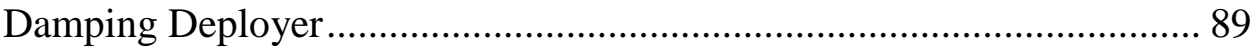

Isolating Deployer from Launch Vehicle ............................................ 90

Isolating CubeSat from Deployer ................................................... 92

P-POD Design Concept ..................................................................... 94

Selected Constraint and Mitigation Trade …........................................... 94

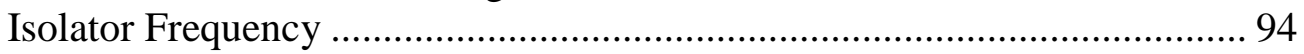

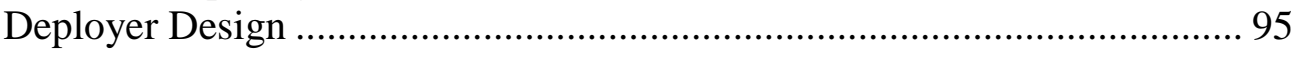

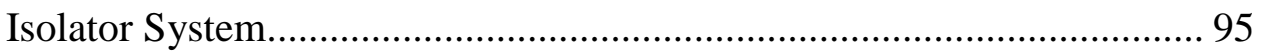

Deployer Resonant Frequencies .................................................... 96

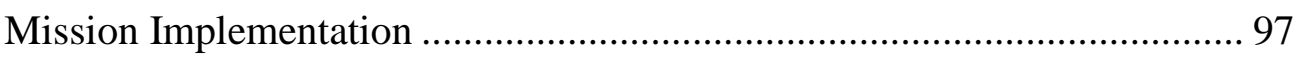

Characterization of Isolator Systems .............................................. 97

Choose Mission Specific Isolator System............................................ 98

CHAPTER 6: Proof of Concept for Isolation at Deployer Rail Interface ....................... 99

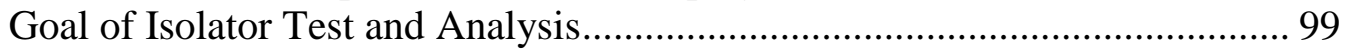

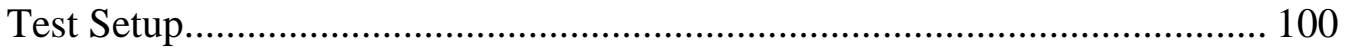

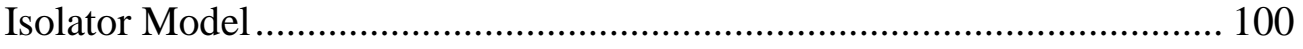

Vibration Equipment................................................................. 101

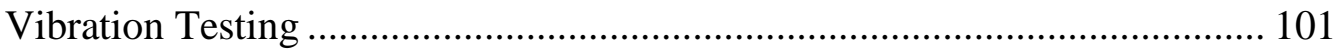

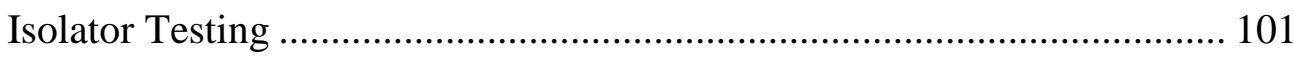

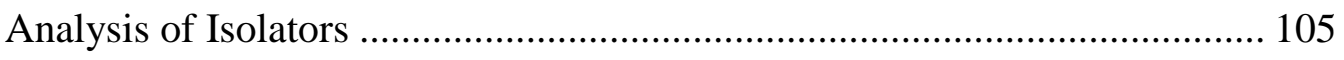

Characterization of Isolator Frequency and Damping Ratio ..................... 105

Comparison to SDOF Model .................................................................. 106

SDOF Model from Sine Sweep Data............................................... 106

Revised SDOF Isolator Model........................................................ 109

Isolator Effectiveness ....................................................................... 112

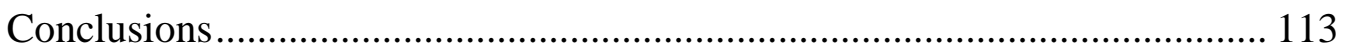

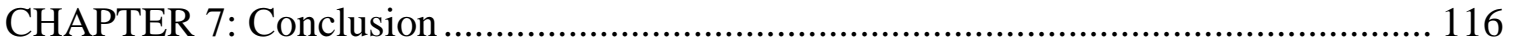


CubeSat-Deployer Model ........................................................................ 116

Proposed Design Concept for Future Deployer .......................................... 118

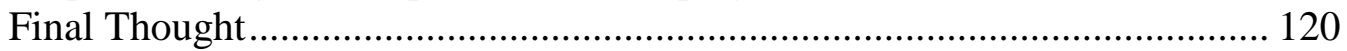

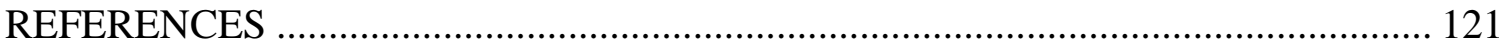

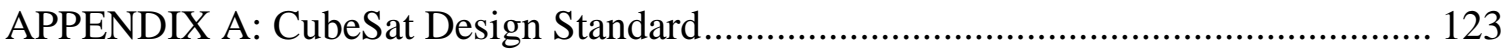

APPENDIX B: Sample Integrated P-PODs........................................................... 126

APPENDIX C: FEA from CubeSat Mass Model and Test CubeSat ............................ 129

APPENDIX D: Vibration Test Setup and Data from Chapter 3 ................................. 135

APPENDIX E: Vibration Test Setup and Data from Chapter 6 .................................. 194 


\section{LIST OF TABLES}

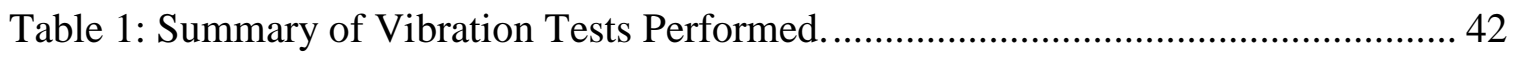

Table 2: CubeSat response to GEVS acceptance profile in different deployers.............. 81

Table 3: CubeSat response to GEVS random vibration profile for fixed

(beginning of test) and isolated (end of test) CubeSats in different deployers................ 84

Table 4: Natural frequency and damping ratios from isolator sine sweep tests. ........... 105

Table 5: Summary of actual versus theoretical isolated CubeSat response from

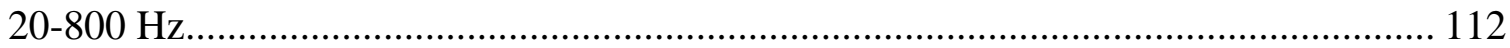




\section{LIST OF FIGURES}

Figure 1: CP-6 is a CubeSat designed by PolySat at Cal Poly. ...................................... 2

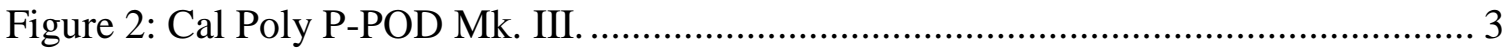

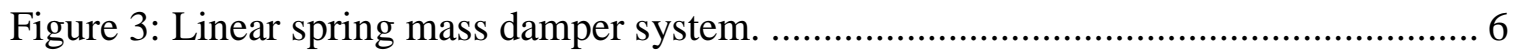

Figure 4: Block diagram of an acceleration FRF....................................................... 8

Figure 5: Diagram of input acceleration to output acceleration load path........................ 9

Figure 6: Amplification and Isolation regions in the frequency domain....................... 10

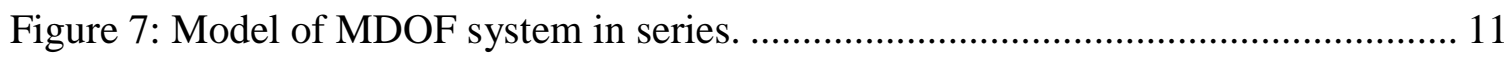

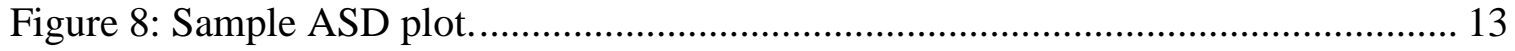

Figure 9: Schematic of isolated structure or payload (credit: Marekich 2012)............... 15

Figure 10: FRF comparison of damped and isolated structure................................... 16

Figure 11: Selected random vibration profiles for CubeSat deployers.......................... 18

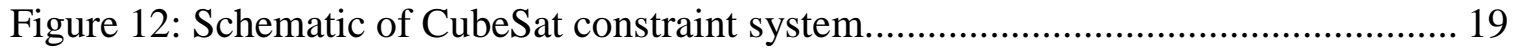

Figure 13: CubeSat response in P-POD and TestPOD........................................... 21

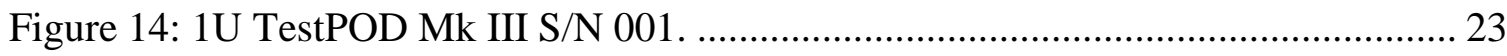

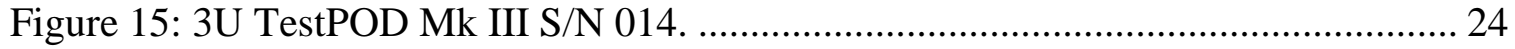

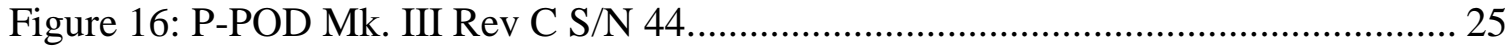

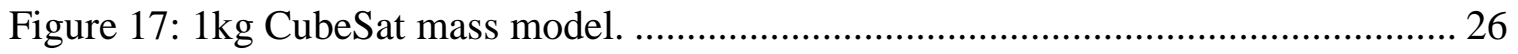

Figure 18: First mode shape of CubeSat mass model with free-free constraint at

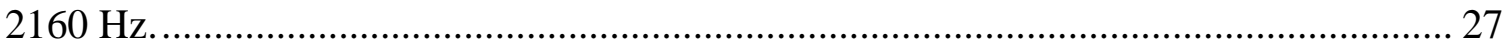

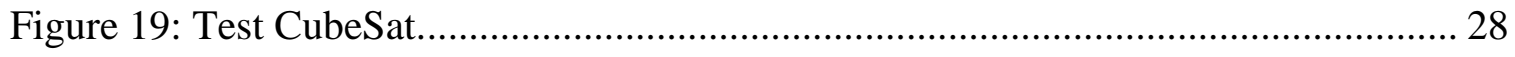

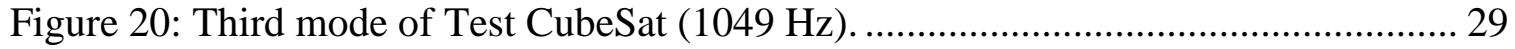

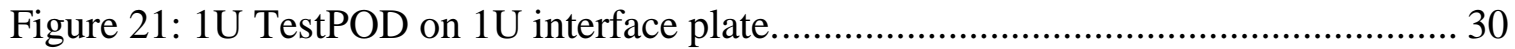

Figure 22: 3U TestPOD attached to table with two interface plates............................ 31

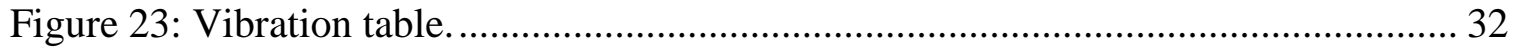

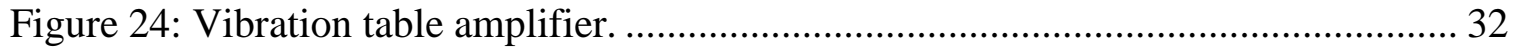

Figure 25: Single axis control accelerometer (PCB M/N 353B04) ............................. 33

Figure 26: Three axis response accelerometer (PCB M/N 354C10). ............................ 33 
Figure 27: Example of accelerometer attachment to test article.................................. 34

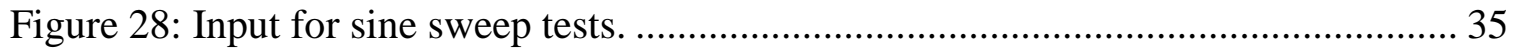

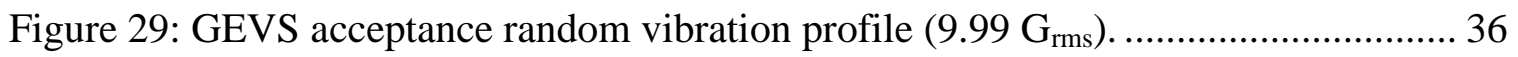

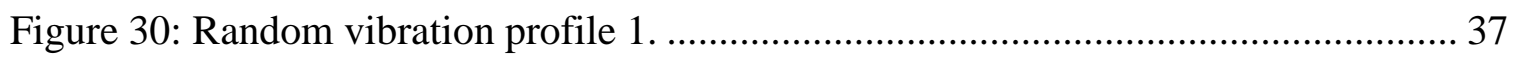

Figure 31: Accelerometer placement on $1 \mathrm{U}$ interface plate for $\mathrm{X}$ axis verification......... 38

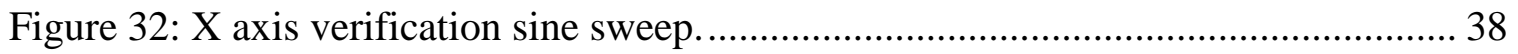

Figure 33: Accelerometer placement on $1 \mathrm{U}$ interface plate for $\mathrm{Y}$ axis verification......... 39

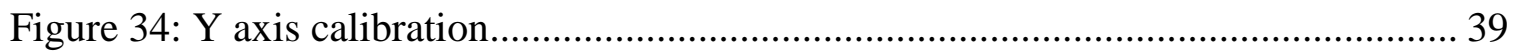

Figure 35: Accelerometer placement on $3 \mathrm{U}$ interface plate for $\mathrm{Z}$ axis verification. ........ 40

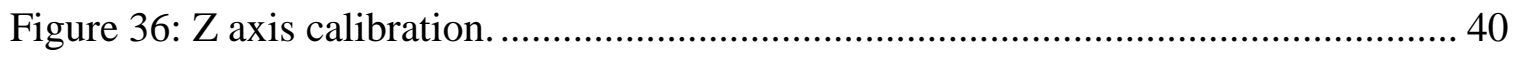

Figure 37: $\mathrm{Z}$ axis vibration data of a $1 \mathrm{~kg}$ CubeSat mass model in a $1 \mathrm{U}$ TestPOD (test numbers 2 and 3 )

Figure 38: $\mathrm{Z}$ axis vibration data from three $1 \mathrm{~kg}$ mass models integrated into a $3 \mathrm{U}$

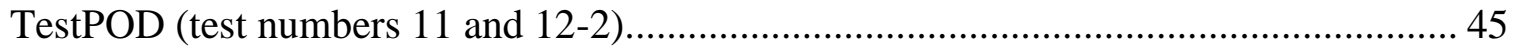

Figure 39: $\mathrm{Z}$ axis vibration data from three $1 \mathrm{~kg}$ mass models integrated into a PPOD (test numbers 17 and 18-2).

Figure 40: $\mathrm{Z}$ axis transmissibility versus frequency for CubeSats in P-POD

subject to Random Vibration Profile 1 and GEVS Random Vibration Profile (test numbers 18-2 and 19).

Figure 41: Fixed and unfixed CubeSat response in $\mathrm{Z}$ axis of $1 \mathrm{U}$ TestPOD (test numbers 3 and 4). 48

Figure 42: Schematic of how spring plungers affect the CubeSat constraint. 50

Figure 43: $\mathrm{X}$ axis vibration data for a $1 \mathrm{~kg}$ CubeSat mass model integrated into a $1 \mathrm{U}$ TestPOD with spring plungers both dialed in tight and backed out (test numbers 6, 8-1 and 9-1).

Figure 44: $\mathrm{X}$ axis sine sweep data for $1 \mathrm{~kg}$ CubeSat mass model integrated into a $1 \mathrm{U}$ TestPOD with spring plungers both dialed in tight and backed out (test numbers 5, 8-2 and 9-2).

Figure 45: $\mathrm{X}$ axis vibration data for a $1 \mathrm{~kg}$ CubeSat mass model integrated into a $1 \mathrm{U}$ TestPOD with standard spring plunger integration (test number 7). 
Figure 46: $\mathrm{X}$ axis vibration data for a $1 \mathrm{~kg}$ CubeSat mass model integrated into a

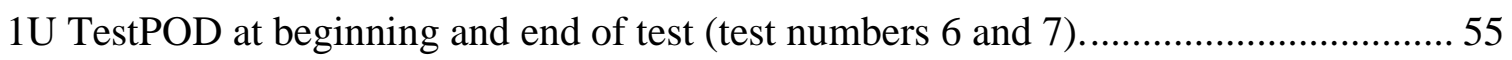
Figure 47: $\mathrm{X}$ axis vibration data for three $1 \mathrm{~kg}$ CubeSat mass models integrated into a 3U TestPOD with standard spring plunger integration (test number 15-2)........... 56 Figure 48: $\mathrm{X}$ axis vibration data for three $1 \mathrm{~kg}$ CubeSat mass models integrated into a $3 \mathrm{U}$ TestPOD at beginning and end of test (test numbers 14 and 15-2). 57

Figure 49: $\mathrm{X}$ axis vibration data for three $1 \mathrm{~kg}$ CubeSat mass models integrated into a P-POD with standard spring plunger integration (test number 22-2)..... 58 Figure 50: $\mathrm{X}$ axis vibration data for three $1 \mathrm{~kg}$ CubeSat mass models integrated into a P-POD at beginning and end of test (test numbers 21 and 22-2)........................ 59 Figure 51: $\mathrm{Z}$ axis load path from vibration input to CubeSat load. ................................ 61 Figure 52: CubeSat response compared to SDOF model $(\boldsymbol{\omega n}=\mathbf{5 4 0} \zeta=.06)$ in a $1 \mathrm{U}$ TestPOD (test number 3 ).

Figure 53: CubeSat response compared to SDOF model $(\boldsymbol{\omega n}=\mathbf{3 2 0} \zeta=.12)$ in a $3 \mathrm{U}$ TestPOD (test number 12-2). 64

Figure 54: CubeSat response compared to SDOF model $(\boldsymbol{\omega n}=\mathbf{4 0 0} \zeta=\mathbf{0 . 1})$ in a P-POD (test number 18-2). 65

Figure 55: Block diagram for $\mathrm{X}$ and $\mathrm{Y}$ axes load path.

Figure 56: Fixed CubeSat response compared to SDOF model $(\boldsymbol{\omega n}=\mathbf{6 0 0} \zeta=$

0.12 ) in $\mathrm{X}$ axis of a $1 \mathrm{U}$ TestPOD (test number 8-1) 68

Figure 57: Unconstrained CubeSat response compared to SDOF isolator model $(\boldsymbol{\omega} n=120 \zeta=.8)$ in $X$ axis of a $1 U$ TestPOD (test number 9-1). 70

Figure 58: Two SDOF FRFs used to approximate the CubeSat response in the $\mathrm{X}$ axis of the $1 \mathrm{U}$ TestPOD.

Figure 59: CubeSat response at beginning of test in 3U TestPOD compared to SDOF deployer model $(\boldsymbol{\omega} n=\mathbf{2 2 0} \zeta=.3)$ (test number 15-2). 74

Figure 60: CubeSat response at beginning of test in P-POD compared to SDOF deployer model $(\boldsymbol{\omega} n=130 \zeta=.14)($ test number $22-2)$. 75

Figure 61: CubeSat response at end of test in $\mathrm{X}$ axis of $3 \mathrm{U}$ TestPOD compared to SDOF isolator model $(\boldsymbol{\omega n}=\mathbf{1 2 0} \zeta=\mathbf{1})($ test number $15-2)$. 76 
Figure 62: CubeSat response at end of test in $\mathrm{X}$ axis of P-POD compared to

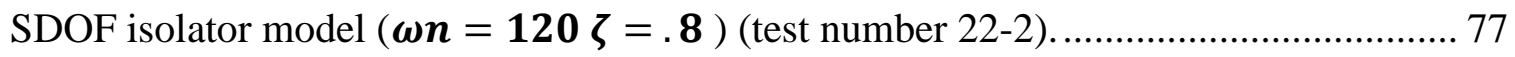

Figure 63: $\mathrm{Z}$ axis CubeSat response in $1 \mathrm{U}$ TestPOD, 3U TestPOD and P-POD

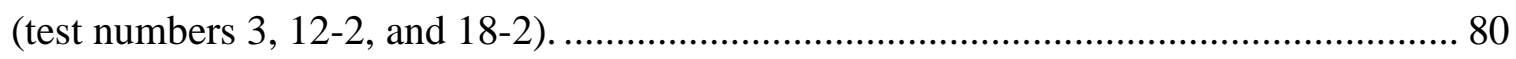

Figure 64: $\mathrm{X}$ axis CubeSat response in 1U TestPOD, 3U TestPOD and P-POD

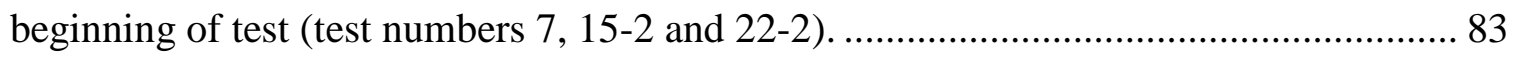

Figure 65: $\mathrm{X}$ axis CubeSat response in 1U TestPOD, 3U TestPOD and P-POD

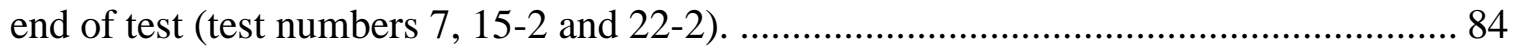

Figure 66: Example of how damping mitigates deployer amplification for

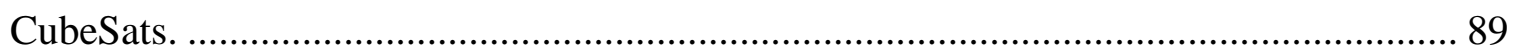

Figure 67: Example of how isolation could mitigate vibration loads........................... 91

Figure 68: Comparison of fixed, unconstrained and isolated CubeSat response.............. 93

Figure 69: Schematic of concept for constraining CubeSat in future deployer............... 94

Figure 70: Location where removable rails and isolator plates would be in future

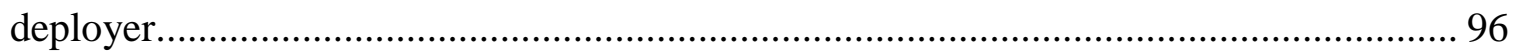

Figure 71: Example of isolation system characterization chart. .................................. 97

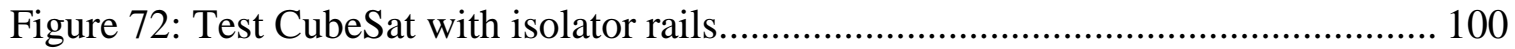

Figure 73: Test setup for three $\mathrm{X}$ axis isolation tests.............................................. 102

Figure 74: Isolated CubeSat response to random vibration profile. ............................ 103

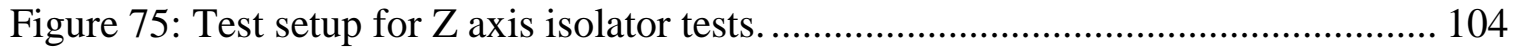

Figure 76: Isolated CubeSat response for comparison case in $\mathrm{Z}$ axis of $1 \mathrm{U}$

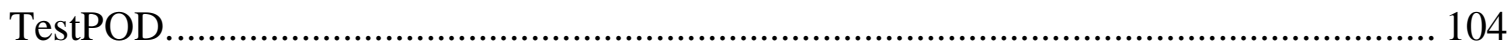

Figure 77: Isolator frequency versus CubeSat mass............................................. 106

Figure 78: SDOF model of .42 kg Test CubeSat using data from Sine Sweep test........ 107

Figure 79: SDOF model of .97 kg Test CubeSat using data from Sine Sweep test........ 107

Figure 80: SDOF model of $1.30 \mathrm{~kg}$ Test CubeSat using data from Sine Sweep test..... 108

Figure 81: SDOF model of $1.30 \mathrm{~kg}$ Test CubeSat in $\mathrm{Z}$ axis using data from Sine

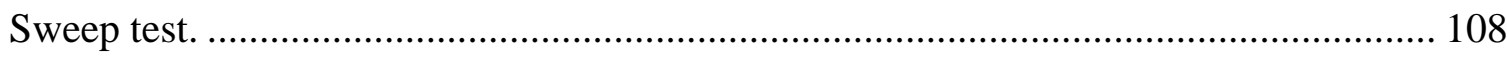

Figure 82: SDOF model of .42 kg Test CubeSat in X axis using revised parameters. 
Figure 83: SDOF model of $.97 \mathrm{~kg}$ Test CubeSat in $\mathrm{X}$ axis using revised parameters.

Figure 84: SDOF model of $1.3 \mathrm{~kg}$ Test CubeSat in $\mathrm{X}$ axis using revised

parameters.

Figure 85: SDOF model of $1.30 \mathrm{~kg}$ Test CubeSat in $\mathrm{Z}$ axis using revised parameters.

Figure 86: Isolated CubeSat response with fixed CubeSat response. 113

Figure 87: 1U CubeSat Design Standard. 124

Figure 88: 3U CubeSat Design Standard. 125

Figure 89: 3 P-PODs integrated on Vega Launch Vehicle. 126

Figure 90: 8 P-PODs on NPS CuL. 127

Figure 91: 3 P-PODs integrated on Delta II. 128

Figure 92: First mode of CubeSat mass model $(2160 \mathrm{~Hz})$. 129

Figure 93: Convergence study for CubeSat mass model. 130

Figure 94: Mode 3 at 1,049 Hz. 131

Figure 95: Mode 5 at 1,443 $\mathrm{Hz}$. 132

Figure 96: Mode 7 at 1,771 Hz. 133

Figure 97: Convergence study for FEM of Test CubeSat. 134

Figure 98: Setup for test numbers 1 and 2. 137

Figure 99: Raw data from test number 1 138

Figure 100: Raw data from test number 2. 139

Figure 101: Setup for test number 3. 140

Figure 102: Raw data from test 3. 141

Figure 103: Setup for test 4. 142

Figure 104: Raw data from test 4. 143

Figure 105: Setup for tests 5 and 6. 144

Figure 106: Raw data from test number 5. 145

Figure 107: Raw data from test 6. 146

Figure 108: Setup for tests 7, 8-1, 8-2, 9-1, and 9-2 (spring plungers were dialed in differently for these tests, but cannot be distinguished through photos). 147 


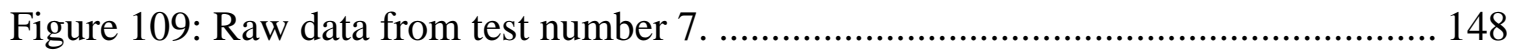

Figure 110: Time varying data from test number 7 ................................................ 149

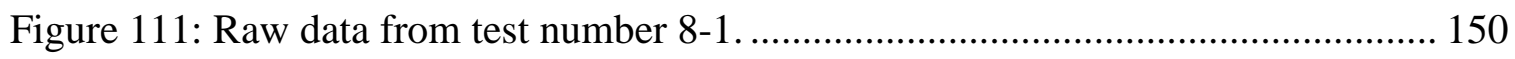

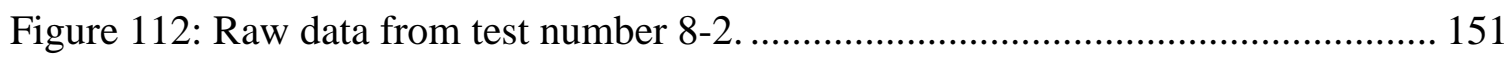

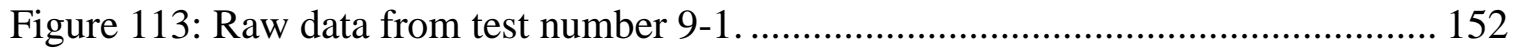

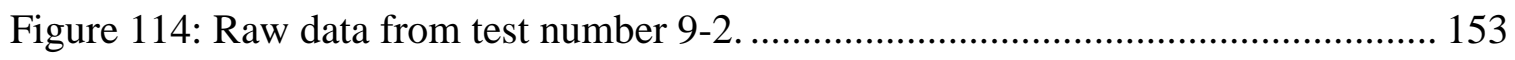

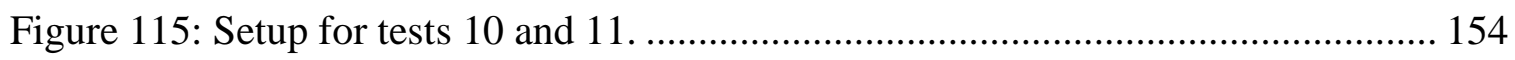

Figure 116: Raw data from test 10....................................................................... 155

Figure 117: Raw data from test 11..................................................................... 156

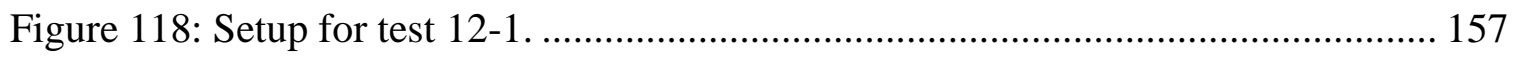

Figure 119: Raw data from test 12-1 ................................................................... 158

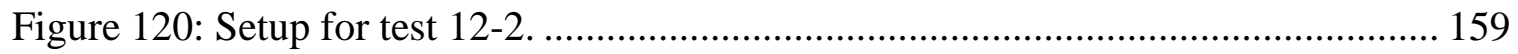

Figure 121: Raw data from test 12-2.................................................................. 160

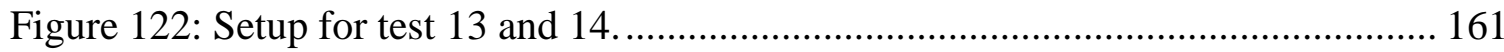

Figure 123: Raw data from test 13 ........................................................................ 162

Figure 124: Raw data from test 14........................................................................ 163

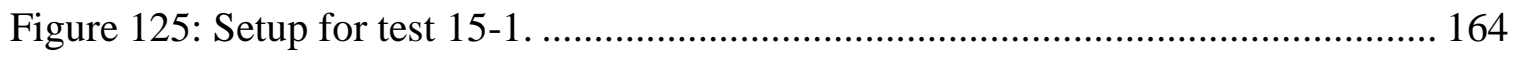

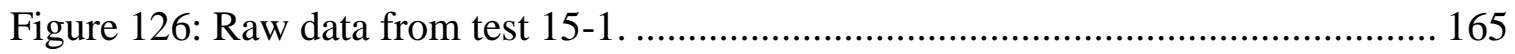

Figure 127: Setup for test 15-2. .......................................................................... 166

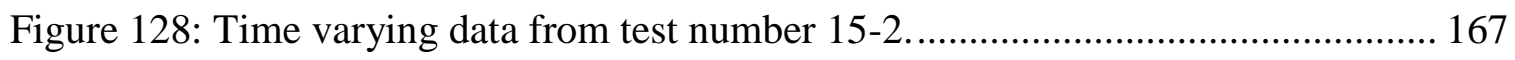

Figure 129: Raw data from test 15-2. …………………………………………... 168

Figure 130: Setup for test numbers 16 and 17 ...................................................... 169

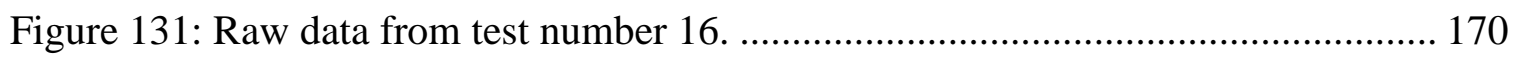

Figure 132: Raw data from test number 17. .............................................................. 171

Figure 133: Setup for test number 18-1............................................................. 172

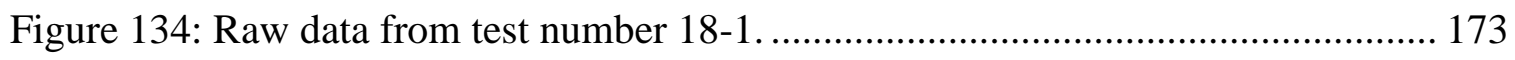

Figure 135: Setup for test numbers 18-2 and 19...................................................... 174

Figure 136: Raw data from test 18-2. …………………..................................... 175

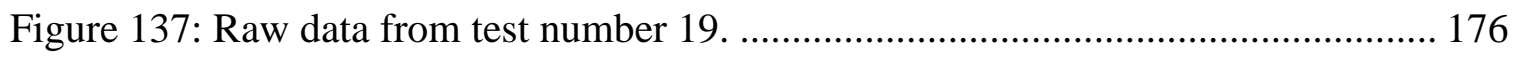

Figure 138: Setup for test number 20 and 21......................................................... 177 


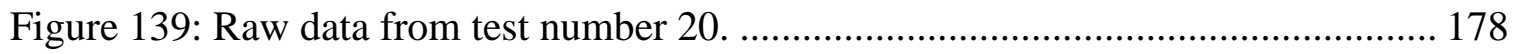

Figure 140: Raw data from test number 21........................................................... 179

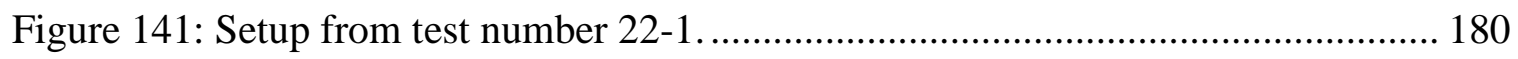

Figure 142: Raw data from test number $22-1$.......................................................... 181

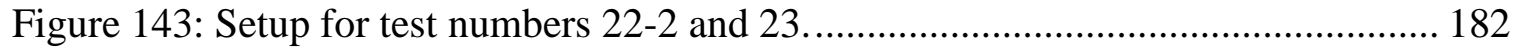

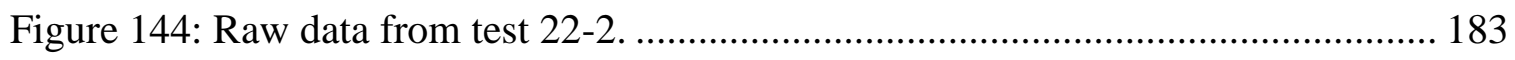

Figure 145: Time varying data from test number 22-2 .......................................... 184

Figure 146: Raw data from test number 23. ......................................................... 185

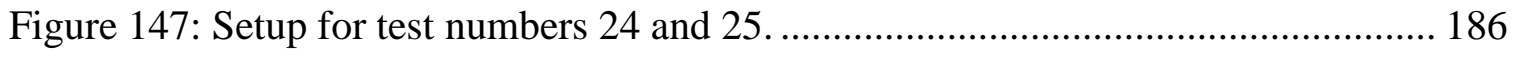

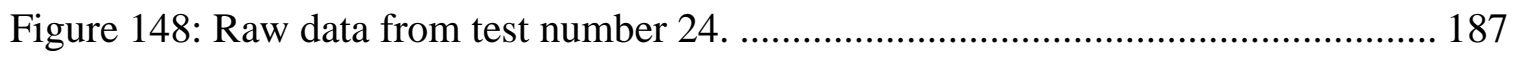

Figure 149: Raw data from test number 25....................................................... 188

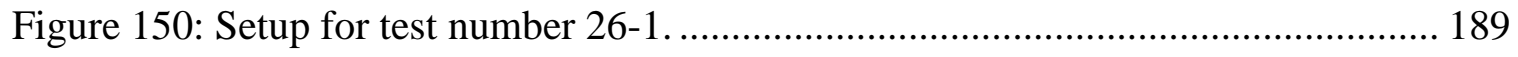

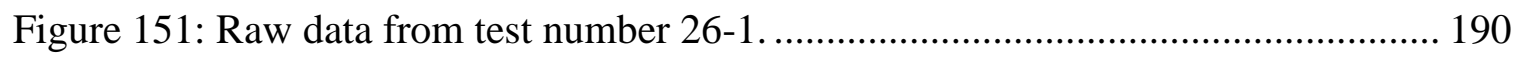

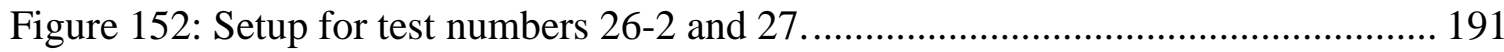

Figure 153: Raw data from test number 26. ......................................................... 192

Figure 154: Raw data from test number 27...................................................... 193

Figure 155: Raw data from $.42 \mathrm{~kg}$ isolated CubeSat $\mathrm{X}$ axis sine sweep. ...................... 195

Figure 156: Raw data from $.97 \mathrm{~kg}$ isolated CubeSat X axis sine sweep. ...................... 196

Figure 157: Raw data from 1.33 kg isolated CubeSat X axis sine sweep. .................... 197

Figure 158: $\mathrm{Z}$ axis sine sweep test of isolated $1.33 \mathrm{~kg}$ Test CubeSat.......................... 198 


\section{CHAPTER 1: Introduction}

\section{The CubeSat Program}

CubeSats are a class of Nanosatellite that conform to a specific standard set by California Polytechnic State University, San Luis Obispo (Cal Poly). This standard was established over a decade ago with the goal of providing universities with the opportunity to launch educational satellites as secondary payloads. The purpose of the CubeSat standard is to ensure CubeSats have a set interface to launch vehicles that minimizes risk to the primary payload mission. This platform provides launch vehicle providers and primary satellite customers with the confidence necessary to allow students consistent access to space.

The CubeSat standard states that one CubeSat Unit (1U) is a 10 centimeter cube with a maximum mass of 1.33 kilograms. The $1 \mathrm{U}$ CubeSat can be expanded to $1.5 \mathrm{U}, 2 \mathrm{U}$ or 3U CubeSats. The CubeSat structure is standardized for integration into the P-POD, or similar orbital deployers, through a rail interface. Standards also regulate CubeSat power, propulsion, communication and other subsystems to mitigate risks to the primary payload. The CubeSat standard is shown in Appendix A. An example of a CubeSat designed at Cal Poly is shown in Figure 1. 


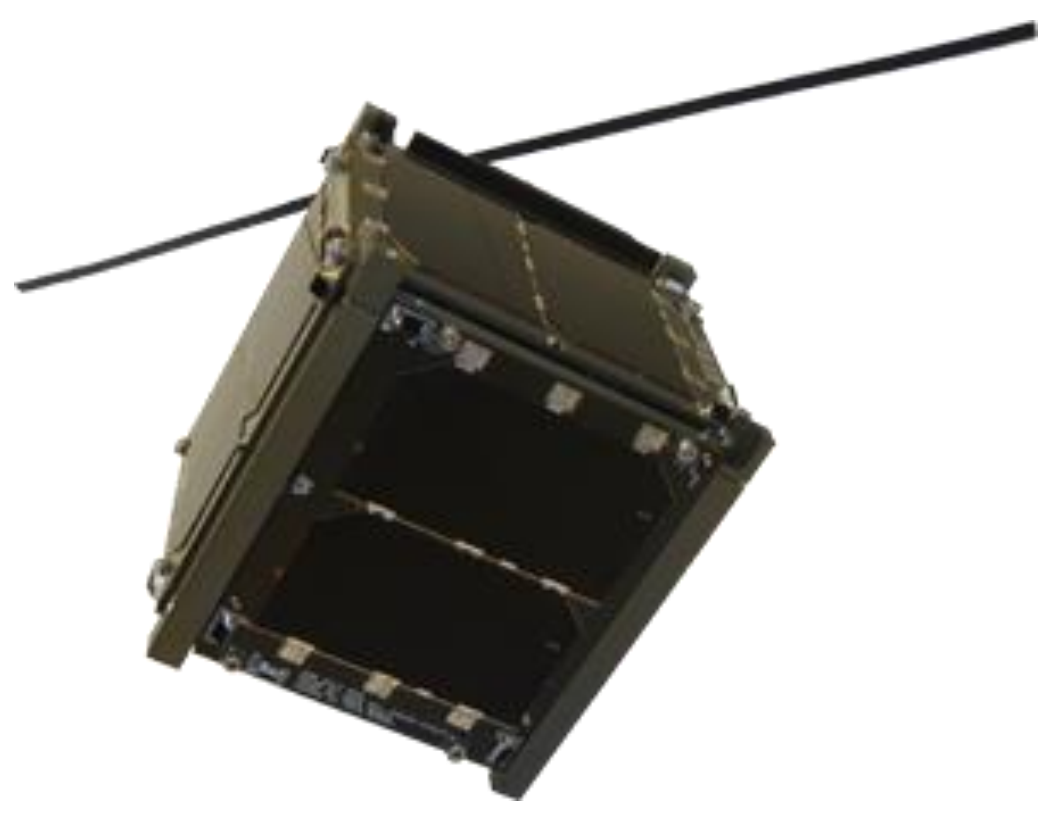

Figure 1: CP-6 is a CubeSat designed by PolySat at Cal Poly.

CubeSat deployers are responsible for providing an interface for CubeSats to the launch vehicle, protecting the primary payload and launch vehicle from CubeSats during launch, and deploying the CubeSat on orbit. Currently, there are several CubeSat deployers available. The Cal Poly P-POD is the original deployer, developed by the same team that created the CubeSat standard. P-PODs have obtained flight heritage with launches including Delta II, Atlas V, Taurus XL, Vega and Falcon 9. The P-POD Mk. III is shown in Figure 2. Appendix B shows P-PODs integrated to launch vehicles for selected missions. 


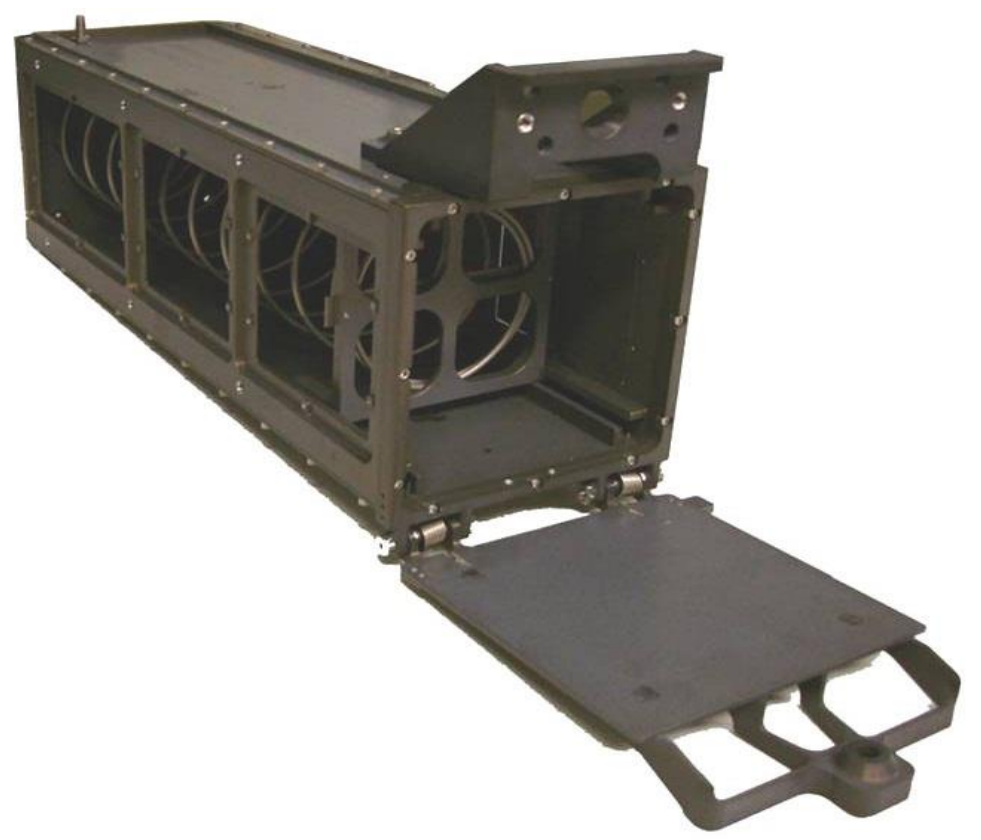

Figure 2: Cal Poly P-POD Mk. III.

While CubeSats were initially intended for educational purposes, commercial and government developers have become increasingly active. With the recent decline in federal spending, CubeSats have become an attractive option for government agencies such as NASA and the Department of Defense. Commercial companies are also developing their own CubeSats, utilizing cost effective access to space to accomplish their mission requirements. Missions have included biology experiments, Earth photography and space weather monitoring. CubeSats also offer a low cost way to test new spacecraft components or architectures.

CubeSats have become increasingly sophisticated over the past decade.

Developers are increasing the capabilities of CubeSats to fulfill more advanced mission objectives. Advances have been made in CubeSat attitude determination and control, avionics, communications, and propulsion. Formation flight, rendezvous and orbit 
transfers are now possible, creating a wide range of opportunities including CubeSat constellations or even interplanetary missions.

\section{Thesis Motivation and Goal}

CubeSats and their deployers are subject to a dynamic launch environment consisting of random vibration and shock. Loads can be very high, in some missions over $30 \mathrm{G}_{\mathrm{rms}}$. These types of loads can cause CubeSat failures during vibration testing or launch, and limit the possibilities for CubeSat payloads and components. In addition, vibration analysis of CubeSats inside deployers is very difficult due to the canisterized constraint system. This requires developers to add margin to their failure analysis or avoid complex subsystems altogether. High, unpredictable vibration loads discourage development of advanced CubeSat systems. If more sophisticated CubeSat subsystems and payloads are to be designed in the future, vibration loads to CubeSats must be better defined, and if possible, reduced.

Previous research has investigated the vibration loads CubeSats see in the Cal Poly P-POD and TestPOD. In "Characterization of the Internal and External Environments of the CubeSat P-POD and Test POD," Veronica Bashbush concluded that different deployer and CubeSat designs affect CubeSat loads (Bashbush 2004). Marissa Brummitt's research came to similar conclusions, as demonstrated in her thesis "Development of CubeSat Vibration Testing Capabilities for the Naval Postgraduate School and Cal Poly San Luis Obispo" which determined that CubeSat vibration loads inside a TestPOD are not the same as loads inside the P-POD (Brummitt 2010). This 
research indicates that deployer and CubeSat designs influence vibration loads for CubeSats, and that vibration analysis of these systems is very difficult.

Continuing on the previous research done at Cal Poly, this thesis attempts to:

- Develop a vibration model for the CubeSat-deployer system

- Understand how deployer designs affect the vibration model and CubeSat vibration loads

- Propose a concept for future deployers

○ Concept must mitigate vibration environment

○ Concept must constrain CubeSats in a manner vibration loads can be easily defined

The goal of the vibration model is to understand how vibration loads are driven into the CubeSat through the canisterized constraint from the P-POD. Development of a CubeSat-deployer system model will lead to a better understanding of how deployer design affects CubeSat loads. This understanding is necessary when designing a future deployer.

The design concept for the future deployer must mitigate the vibration environment while constraining the CubeSat in a manner that is predictable and analyzable. Acceptable vibration mitigation will be defined as a CubeSat response that is less than the vibration input from a random vibration profile. CubeSat response will be considered analyzable if the vibration model is predictable and consistent, and can be characterized through experimentation or linear vibration analysis. 


\section{CHAPTER 2: Preliminary Research}

\section{Vibration Theory}

This section presents the vibration theory, equations and methods used for the dynamic analysis of CubeSat vibration loads in this thesis.

\section{Single Degree of Freedom Spring Mass Damper Model}

The linear single degree of freedom (SDOF) spring mass damper system provides the analyst with a simple and well established model for dynamic analysis.

Figure 3 shows the linear spring mass damper model where $Y$ is the input to the system, $X$ is the response, $k$ is the stiffness, $m$ is the mass and $c$ is the damping.

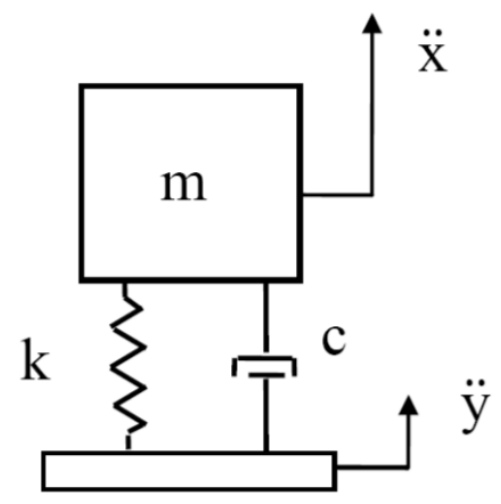

Figure 3: Linear spring mass damper system.

This system can be described by the second-order ordinary differential equation shown in Equation 1. 


$$
p(t)=m \ddot{x}+c \dot{x}+k x \quad \text { Equation } 1
$$

Given an initial velocity and displacement, this differential equation can be solved for the time response of free vibration. Carrying out this calculation shows that the free vibration response of this system is simple harmonic motion that decays at an exponential rate.

The natural frequency of a system, $\omega_{n}$, is the frequency at which it oscillates during free vibration. By neglecting the first-order damping term, the natural frequency can be obtained from Equation 1, and is shown in Equation 2.

$$
\omega_{n}=\sqrt{\frac{k}{m}} \quad \text { Equation 2 }
$$

Damping in the system creates exponential decay during free vibration, with higher values of $c$ lowering the amplitude of oscillation at a much faster rate. The damping ratio is defined as the damping in a system divided by the damping value that would critically damp the system $\left(c_{c r}\right)$. The damping ratio is shown in Equation 3 and the calculation for determining critical damping is shown in Equation 4.

$$
\begin{array}{cc}
\zeta=\frac{c}{c_{c r}} & \text { Equation 3 } \\
c_{c r}=2 m \omega_{n}=2 \sqrt{k m} & \text { Equation 4 }
\end{array}
$$

The natural frequency changes when damping is added to the system. Using the damping ratio, the damped natural frequency, $\omega_{d}$, can be calculated using Equation 5. 


$$
\omega_{d}=\omega_{n} \sqrt{1-\zeta^{2}}
$$

Equation 5

As the damping ratio increases, the natural frequency decreases. This change is usually small, and is often neglected in natural frequency calculations for structures.

\section{Transmissibility and Steady State Frequency Response to Base Excitation}

The majority of the analysis in this thesis pertains to obtaining the response of a structure given a steady state base input. The following provides an overview of the methods used to obtain this response.

\section{Frequency Response Function}

Transmissibility is the ratio of a system's response to an input, and is found by using frequency response functions (FRFs). The FRF is the system's transfer function, and gives the magnitude and phase response as a function of input frequency, $\omega$. FRFs can be used in terms of acceleration, velocity, force, displacement, and other vibration parameters that are functions of frequency. Figure 4 shows a block diagram illustrating an acceleration FRF. Figure 5 shows the load path of the input to the output.

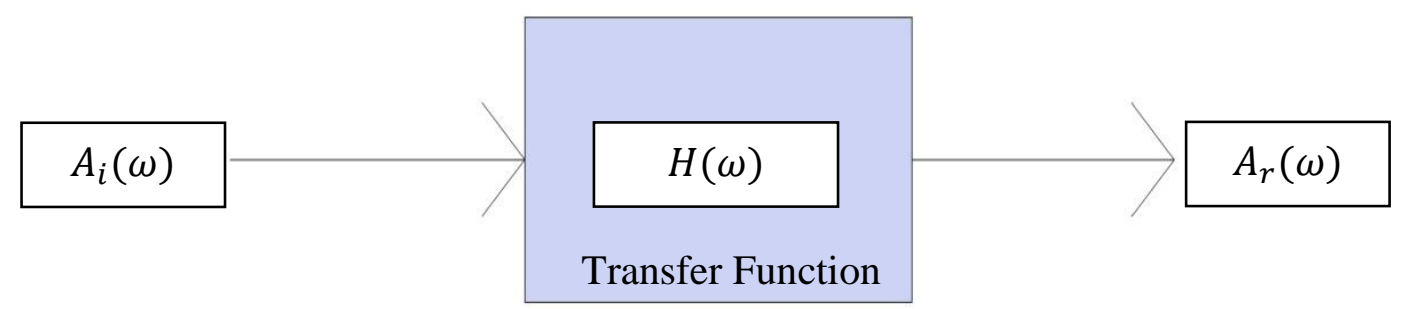

Figure 4: Block diagram of an acceleration FRF. 


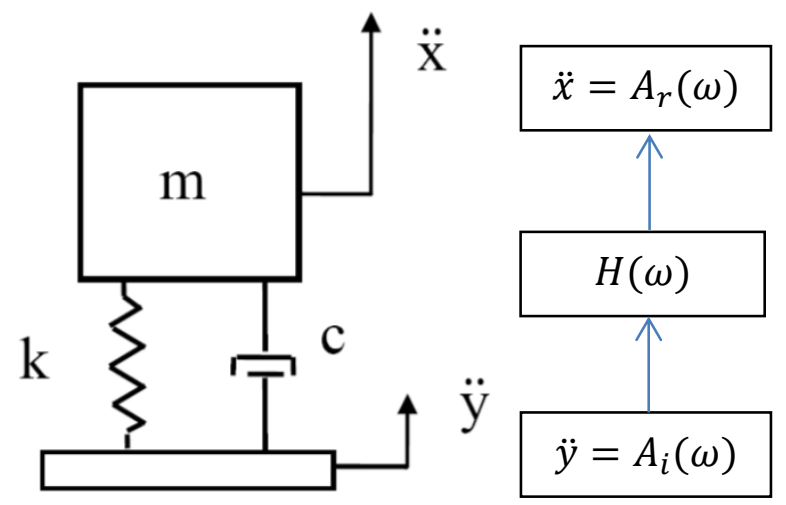

Figure 5: Diagram of input acceleration to output acceleration load path.

The FRF is $H(\omega)$, and is shown in the transfer function block. The input is an acceleration that is a function of frequency, $A_{i}(\omega)$. The response is acceleration as a function of frequency, $A_{r}(\omega)$. The transmissibility is the output over the input, or $\frac{A_{r}(\omega)}{A_{i}(\omega)}$.

The natural frequency and damping ratio are needed to create FRFs for linear spring mass damper systems. Equation 6 shows the acceleration magnitude FRF.

$$
\frac{A_{r}(\omega)}{A_{i}(\omega)}=\sqrt{\frac{1+(2 \zeta r)^{2}}{\left(1-r^{2}\right)^{2}+(2 \zeta r)^{2}}} \quad \text { Equation } 6
$$

In Equation 6,r is the ratio of driving frequency to the system's natural frequency $\left(\frac{\omega}{\omega_{d}}\right)$. Equation 6 is the transfer function shown in Figure 4 and describes the response of the mass from Figure 5. This is the primary equation used to model CubeSat response in this thesis. 


\section{Regions of Amplification and Isolation}

The SDOF acceleration FRF can be divided into two regions: a region of magnification and a region of isolation. Amplification occurs at input frequencies below $\sqrt{2}$ times the system's natural frequency, or $\omega<\sqrt{2} \omega_{n}$. Isolation occurs at frequencies higher than this, or $\omega>\sqrt{2} \omega_{n}$. If the input frequency is much lower than the natural frequency (an octave below is a general rule of thumb), the response is estimated to be the same as the input. Figure 6 illustrates these two regions in the frequency domain.

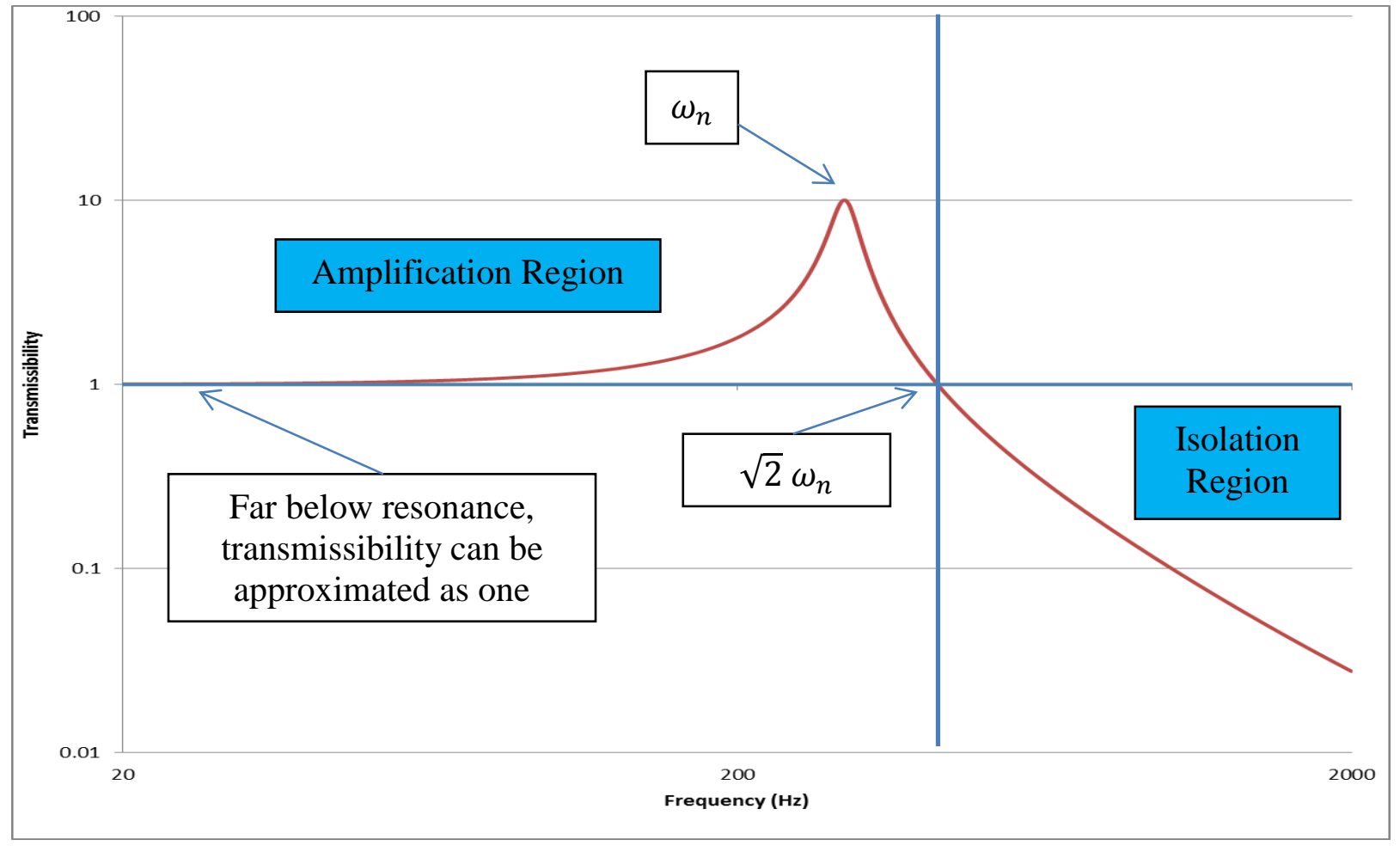

Figure 6: Amplification and Isolation regions in the frequency domain.

The largest amplification, or peak transmissibility, occurs at the system's natural frequency. The peak transmissibility, $Q_{\text {peak }}$, is a function of the damping ratio, and is shown in Equation 7. 


$$
Q_{\text {peak }}=\frac{1}{2 \zeta}
$$

Equation 7

\section{Multiple Degrees of Freedom}

Multiple Degree of Freedom (MDOF) systems are more complicated to model than the SDOF systems discussed earlier. MDOF analysis is simplified in this thesis by assuming there is no coupling between degrees of freedom. This assumption assumes the natural frequencies of the degrees of freedom are separated by at least one octave. For MDOF systems in series, FRFs are simply multiplied by each other. Figure 7 shows the model for an MDOF system in series, where $H_{1}(\omega)$ is multiplied by $H_{2}(\omega)$ to obtain the total system transfer function. Parallel degrees of freedom are assumed to not affect each other's FRFs. If coupling does occur, MDOF analysis is not performed.

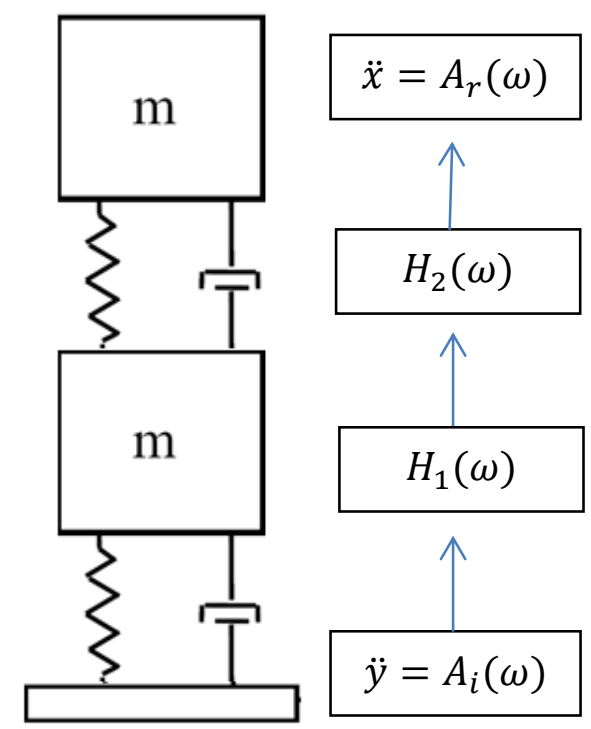

Figure 7: Model of MDOF system in series. 


\section{Random Vibration}

Random vibration consists of a spectrum of frequencies, with the amplitude of these frequencies varying randomly. On launch vehicles, random vibration sources include engine vibration and acoustics. Random vibrations are extremely important to deployers because they usually represent the highest loads.

Statistical methods are used to describe random vibration loads because there is no inherent mathematical way to describe a random vibration time history. $G_{\mathrm{rms}}$ is the root mean square of the accelerations in a random vibration history, and is the most common method of describing the magnitude of random vibration acceleration. The $\mathrm{G}_{\mathrm{rms}}$ is equivalent to the $1 \sigma$ value of acceleration if the mean vibration is zero. This means that, statistically, about $68 \%$ of the acceleration time history will be below the $\mathrm{G}_{\mathrm{rms}}$. The peak response is generally assumed to be $3 \sigma$, but can vary due to the nature of random vibration.

The magnitude of random vibration, or $\mathrm{G}_{\mathrm{rms}}$, generally varies for different frequency bandwidths. For this reason, it is beneficial to divide up the overall $\mathrm{G}_{\mathrm{rms}}$ into smaller frequency bandwidths. This leads analyst to use the unit $\mathrm{Grms}^{2} / \mathrm{Hz}$, or more

commonly $\mathrm{G}^{2} / \mathrm{Hz}_{\mathrm{z}}$ (the "rms" subscript is implied) to describe random vibration amplitude. To calculate this, the overall frequency range is divided into chosen frequency bandwidths. For each of these bandwidths, the $\mathrm{G}_{\mathrm{rms}}$ is calculated, squared, and 
divided by the bandwidth to get the $\mathrm{G}^{2} / \mathrm{Hz}_{\mathrm{z}}$ value. For example, if the $\mathrm{G}_{\mathrm{rms}}$ between 10

$\mathrm{Hz}$ and $20 \mathrm{~Hz}$ is $1.2 \mathrm{~g}$ 's, then the $\mathrm{G}^{2} / \mathrm{Hz}$ value for that $10 \mathrm{~Hz}$ bandwidth is:

$$
\frac{\left(1.2 g^{\prime} s\right)^{2}}{10 \mathrm{~Hz}}=.144^{\mathrm{G}^{2}} / \mathrm{Hz}
$$

The magnitude of $\mathrm{G}^{2} / \mathrm{Hz}$ is assigned to the frequency at the center of its bandwidth. This is plotted against frequency to produce a Power Spectral Density (PSD) or Acceleration Spectral Density (ASD) plot. Figure 8 shows a sample ASD plot.

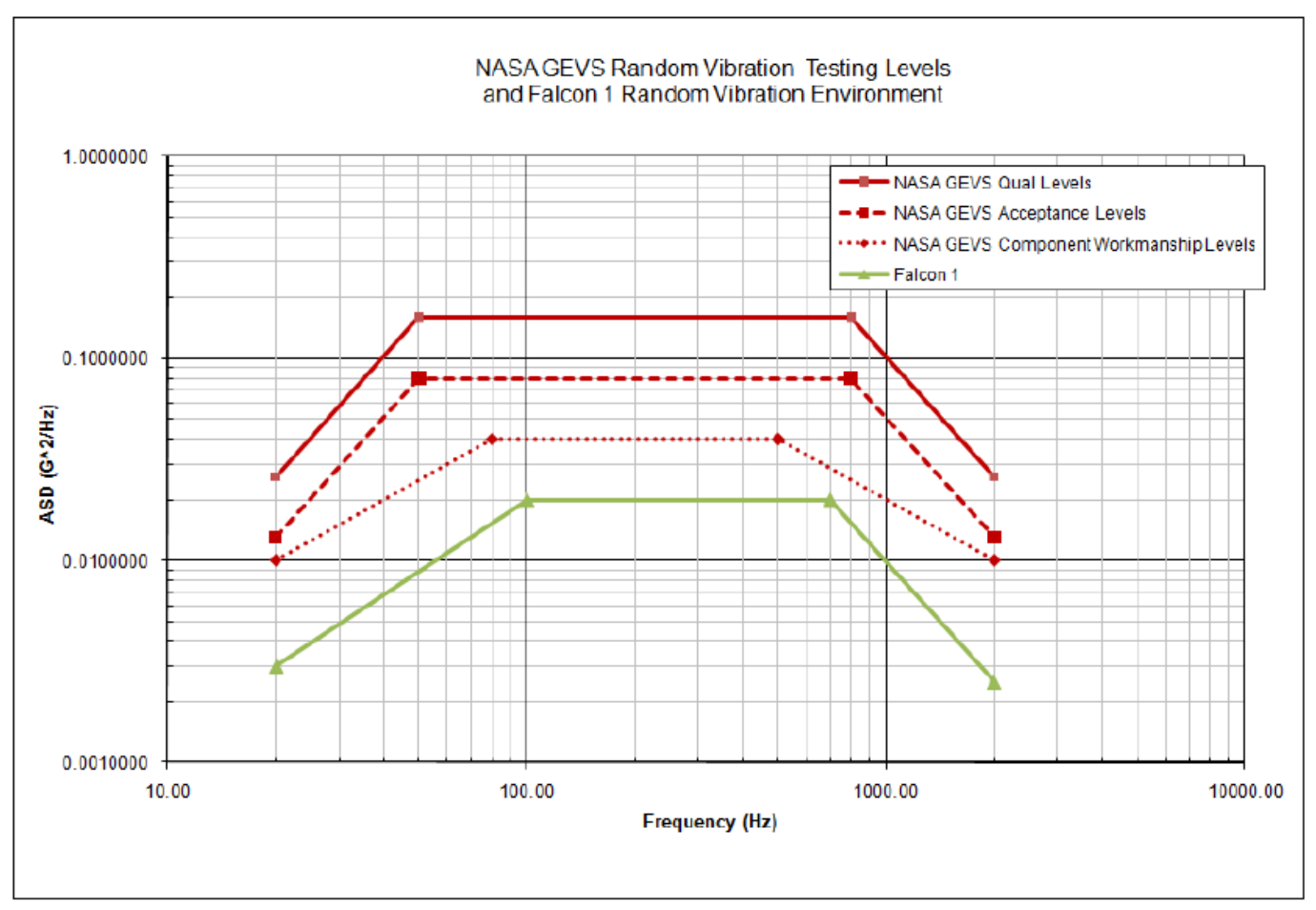

Figure 8: Sample ASD plot.

Page 13 
As shown in the previous sections, understanding the frequency content of vibration is extremely important for obtaining the response of a structure. These units and plots allow the analyst to look at the random vibration $\mathrm{G}_{\mathrm{rms}}$ at different frequencies.

Because ASD units are in $\mathrm{Grms}^{2} /_{\mathrm{Hz}}$, integration of an ASD plot over a

frequency range will give the total $\mathrm{Grms}^{2}$ for that frequency range. To get the total $\mathrm{G}_{\mathrm{rms}}$ from an ASD plot, simply integrate over the entire frequency range and take the square root.

\section{Vibration Mitigation Techniques}

This section outlines the basic vibration mitigation techniques researched for mitigation of the CubeSat dynamic environment. Damping and isolation of structures are discussed, with a comparison between methods provided at the end of the section.

\section{Damping}

Damping takes kinetic energy from vibration and turns it into heat, sound, or some other form of mechanical loss. Dampers or damping material are added to a structure to increase its damping ratio. As shown in Equation 7, the peak transmissibility of a structure is a function of damping ratio. Higher damping ratios lower the peak response at a system's natural frequency. Damping also lowers transmissibility in the entire amplification region. However, damping increases the transmissibility in the isolation region. Therefore, damping is most effective when most of the vibration input is occurring at frequencies in the amplification region. 


\section{Isolation}

Vibration isolation is used to mechanically isolate a structure from a vibration input. Isolators are modeled by adding a spring and damper between the input and the structure, and modeling the structure as the mass for that spring and damper. Figure 9 shows a schematic of how a structure or payload would be modeled when isolators are added.

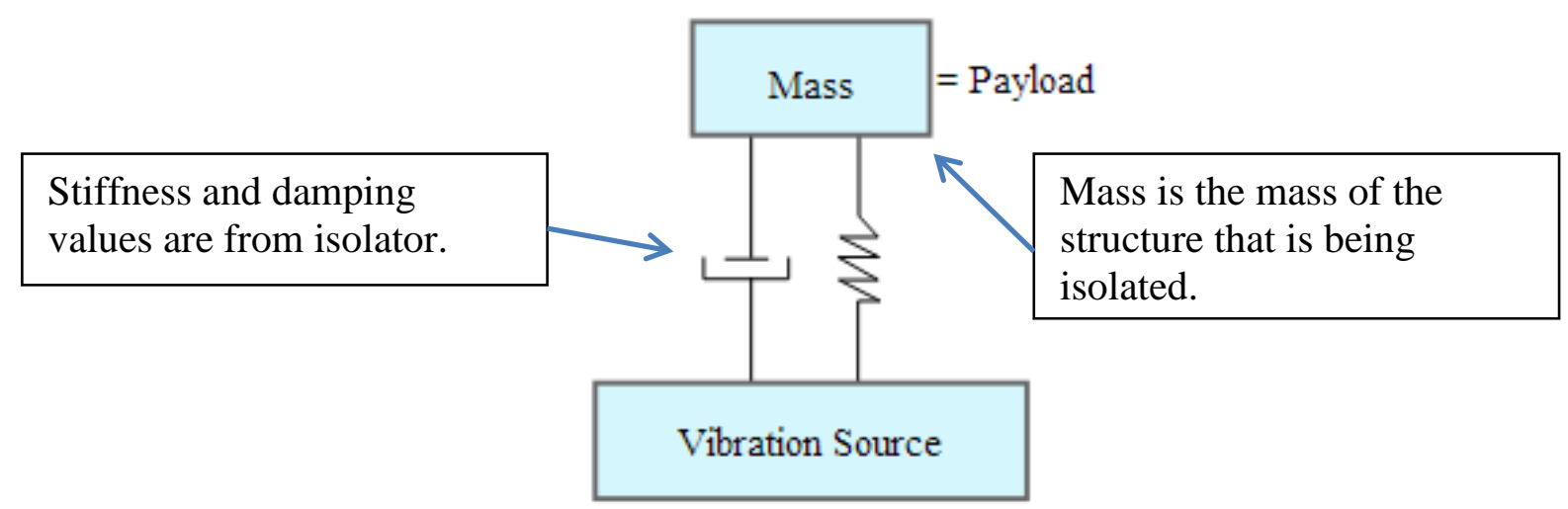

Figure 9: Schematic of isolated structure or payload (credit: Marekich 2012).

The isolator frequency must be far enough below the structure's natural frequency to avoid coupling. The isolated structure's response is assumed to be the response of the mass from the isolator spring mass damper system in Figure 9.

When isolators are used, a structure's region of isolation starts at a much lower frequency than if the isolators where not in place, reducing the transmissibility at the structure's resonant frequency. It is important to note that the isolation region starts at $\sqrt{2}$ times the isolator frequency, and isolators are only effective in the isolation region. Frequencies below $\sqrt{2}$ times the isolator frequency will actually have a higher 
transmissibility than without the isolators. For an isolator to be effective, it must be able to displace more than the input. Without displacement, the isolator will bottom out and become ineffective.

\section{Comparison of Isolation and Damping}

While often used interchangeably, vibration isolation and damping are two different methods of vibration mitigation. Damping attempts to lower the amplification at the resonant peak of a structure by taking away energy from the system, while isolation attempts to mechanically isolate a system from vibration input. Figure 10 illustrates the difference between the FRFs of a damped structure and an isolated structure.

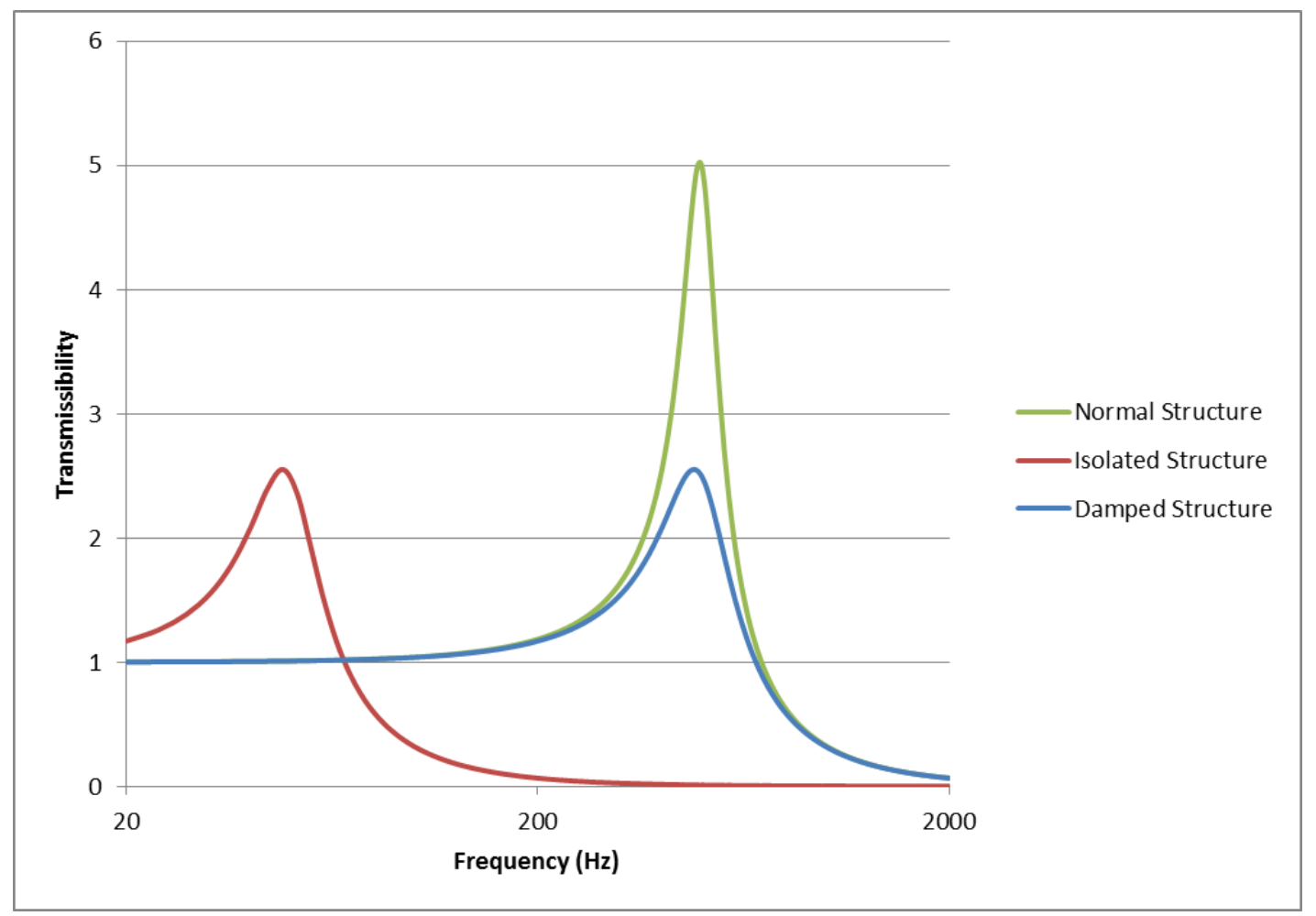

Figure 10: FRF comparison of damped and isolated structure. 
The damped structure has just a slightly lower natural frequency and a smaller peak than the normal structure. The isolated structure has a much lower natural frequency than the normal structure, and has a much larger region of isolation.

\section{CubeSat Loads}

The following section defines deployer loads and outlines the previous research regarding CubeSat loads.

Deployer Loads

Deployer loads are the input to the CubeSat deployer, and are derived by launch vehicle providers. Loads include the random vibration, shock and quasi-static loads. Environmental testing standards usually require CubeSat deployers to be testing to the random vibration profile. If shock loads are high enough, deployers must be tested to the shock profile as well. Quasi-static loads are generally encompassed by the random vibration profile. Figure 11 shows selected random vibration profiles for CubeSat deployers. 


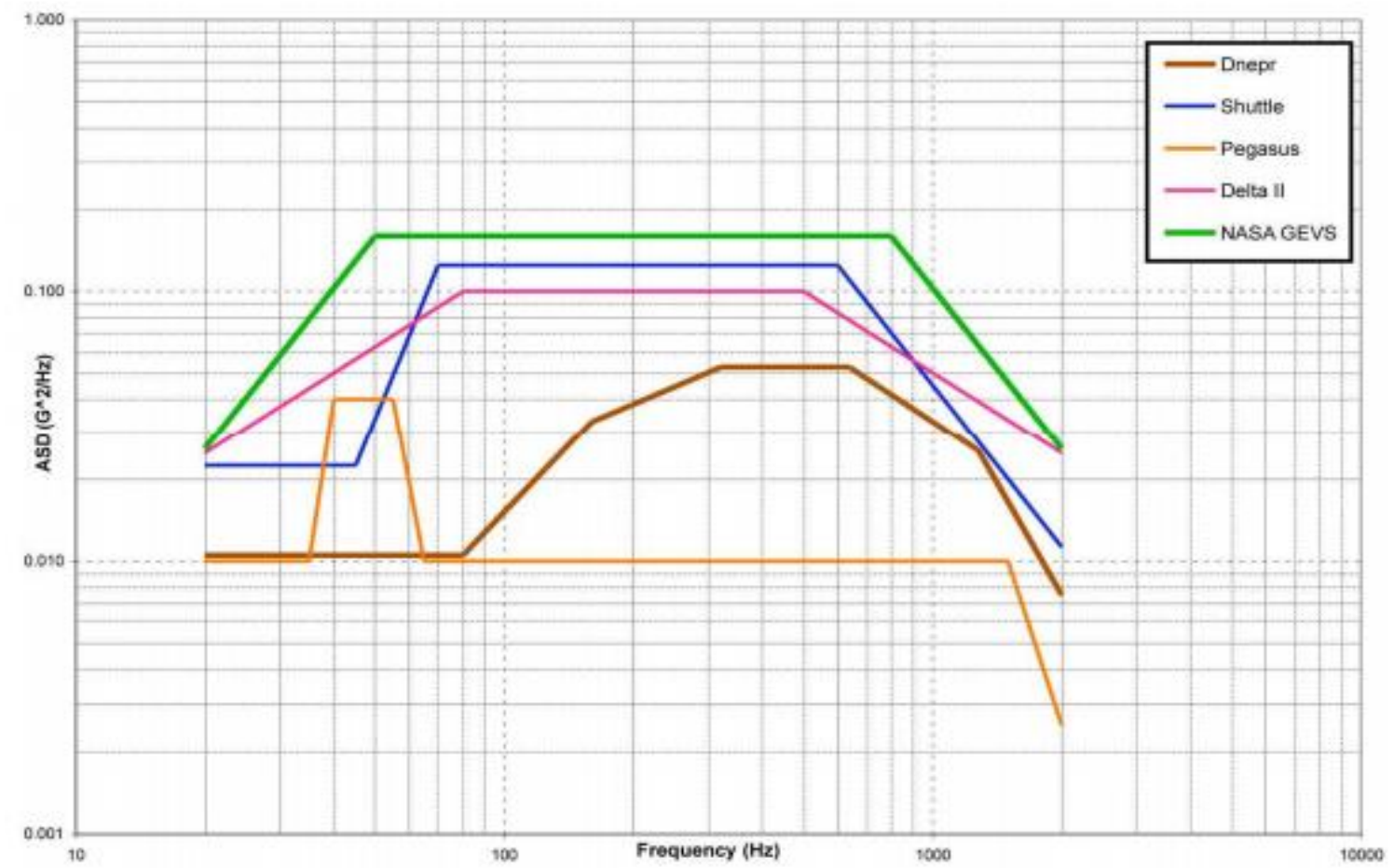

Figure 11: Selected random vibration profiles for CubeSat deployers.

\section{CubeSat Loads}

Unlike deployer loads, CubeSat loads are generally not accurately defined.

Previous research has shown that CubeSat loads are affected by the deployer dynamics and CubeSat design, but the canisterized constraint in the $\mathrm{X}$ and $\mathrm{Y}$ axes makes analysis difficult.

\section{CubeSat Constraint}

CubeSats are constrained by a rail system that has come to be known as a canister constraint system. There are rails on both the deployer and the CubeSats in the $\mathrm{X}$ and $\mathrm{Y}$ axes, allowing the CubeSats to slide freely inside and out of the deployer in the $\mathrm{Z}$ axis during integration and deployment. When the deployer door is closed, spring plungers 
(or a bolt like part) are dialed in to completely constrain the CubeSats in the $\mathrm{Z}$ axis.

Figure 12 shows a cross-section schematic of how CubeSats are constrained.

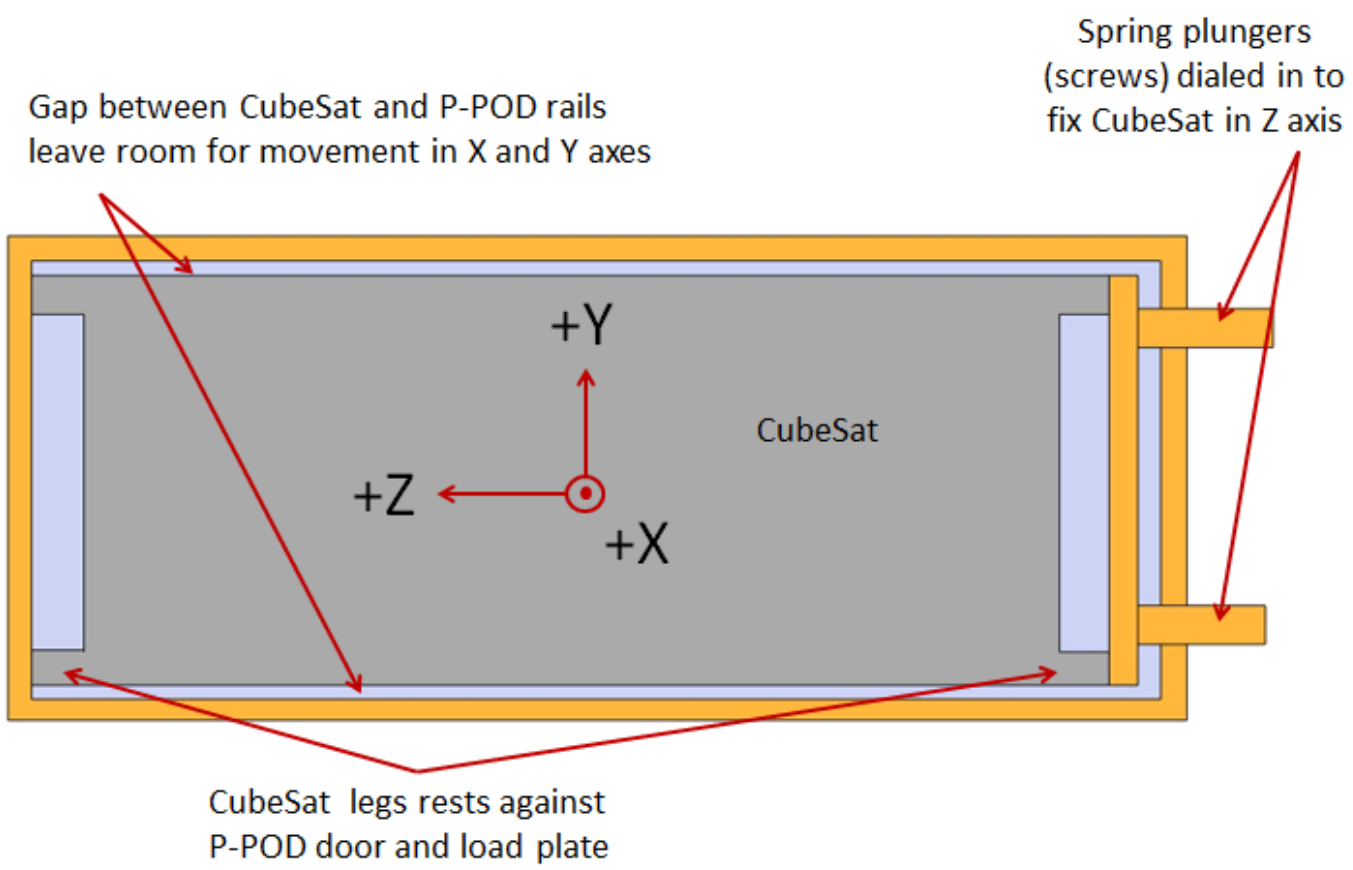

Figure 12: Schematic of CubeSat constraint system.

The canister constraint is useful for many reasons. The gap in the $\mathrm{X}$ and $\mathrm{Y}$ axes and the spring plungers in the $\mathrm{Z}$ provide a way to absorb a wide tolerance needed to make the CubeSat standard possible. This provides a robust interface between CubeSats and the deployer, giving confidence to developers that their CubeSat will be able to fit in the deployer. The canister constraint system allows for the deployment of 3 $1 \mathrm{U}$ CubeSats, $13 \mathrm{U}$ CubeSat, or any other combination of up to $3 \mathrm{U}$ inside the deployer. The rail system deploys CubeSats with a low tumble rate in a predictable direction. The canister constraint allows for a deployer design that completely surrounds the CubeSats, and protects the launch vehicle from any CubeSat failures. Overall, this constraint 
system allows for a simple, reliable design for a CubeSat interface and on-orbit deployment system.

\section{Deployer's Effect on CubeSat Vibration Loads}

While the canister constraint system is very useful for many aspects of the CubeSat mission, it makes vibration analysis difficult. Loads in the $\mathrm{Z}$ axis are easier to predict because the CubeSats are fixed in that axis, but loads in the $\mathrm{X}$ and $\mathrm{Y}$ axes are difficult to determine. Previous research shows that CubeSats in the $\mathrm{Z}$ axis resonate with the deployer. Research has also shown that the deployer resonance has an effect on CubeSats in the $\mathrm{X}$ and $\mathrm{Y}$ axes, but the effect is not as well defined as in the $\mathrm{Z}$ axis.

Because CubeSats respond to their deployer's resonance, CubeSat loads vary when integrated into deployers with different resonant frequencies. Figure 13 shows data from Marissa Brummitt's research illustrating how CubeSat frequency response can differ in different deployers (Brummitt 2010). 


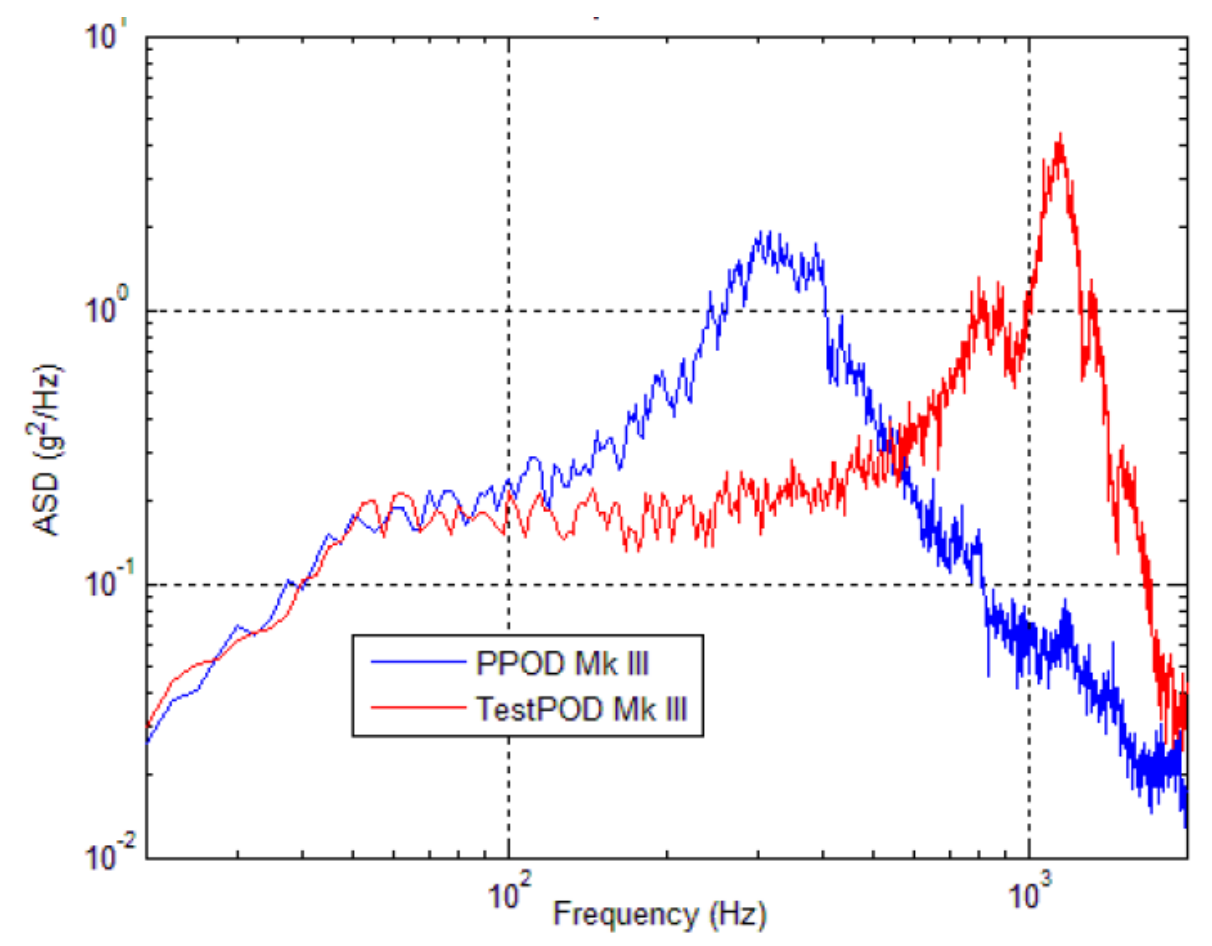

Figure 13: CubeSat response in P-POD and TestPOD.

The CubeSats' peak response is at a lower frequency in the P-POD than in the TestPOD because the TestPOD has a higher resonant frequency than the P-POD.

Previous research has also shown that CubeSat design, including mass and structure stiffness, can affect the resonant frequency of the deployer, and thus the loads the CubeSats see. Stiffer CubeSats raise the natural frequency of the deployer, especially in the $\mathrm{Z}$ axis. CubeSats that are more massive decrease the deployer's natural frequency. The peak response of CubeSats is much lower for CubeSats with more damping. 
CHAPTER 3: Vibration Testing

Continuing the work of previous research done at Cal Poly, vibration tests were run collecting experimental data on CubeSat loads. Because of the difficulty in analyzing the CubeSat-deployer system, vibration testing was done before analysis. This chapter covers the vibration equipment used, the test setups, and the raw data collected from the vibration tests. Analysis and discussion of the vibration data is shown in the following chapter.

Vibration Equipment and Test Setup

\section{Deployers}

A brief overview of the deployers used during testing is provided below. The PPOD is an actual flight deployer, and the $1 \mathrm{U}$ TestPOD and $3 \mathrm{U}$ TestPOD are only used for ground vibration tests. While TestPODs are not flight deployers, they still provide valuable information about how different deployer designs and stiffnesses can affect vibration loads. More information can be found in the user guides for the $1 \mathrm{U}$ TestPOD and 3U TestPOD, which are online at CubeSat.org. Additional information about the PPOD can also be found at CubeSat.org.

$1 U$ TestPOD Mk III

The 1U TestPOD Mk III is the current test deployer used for $1 \mathrm{U}$ CubeSats. The $1 \mathrm{U}$ TestPOD allows for multiple tests with different CubeSats to be run quickly due to 
the ease of integration (they do not require the spring plungers to be staked). Figure 14 shows the 1U TestPOD Mk III S/N 001 used for vibration testing.

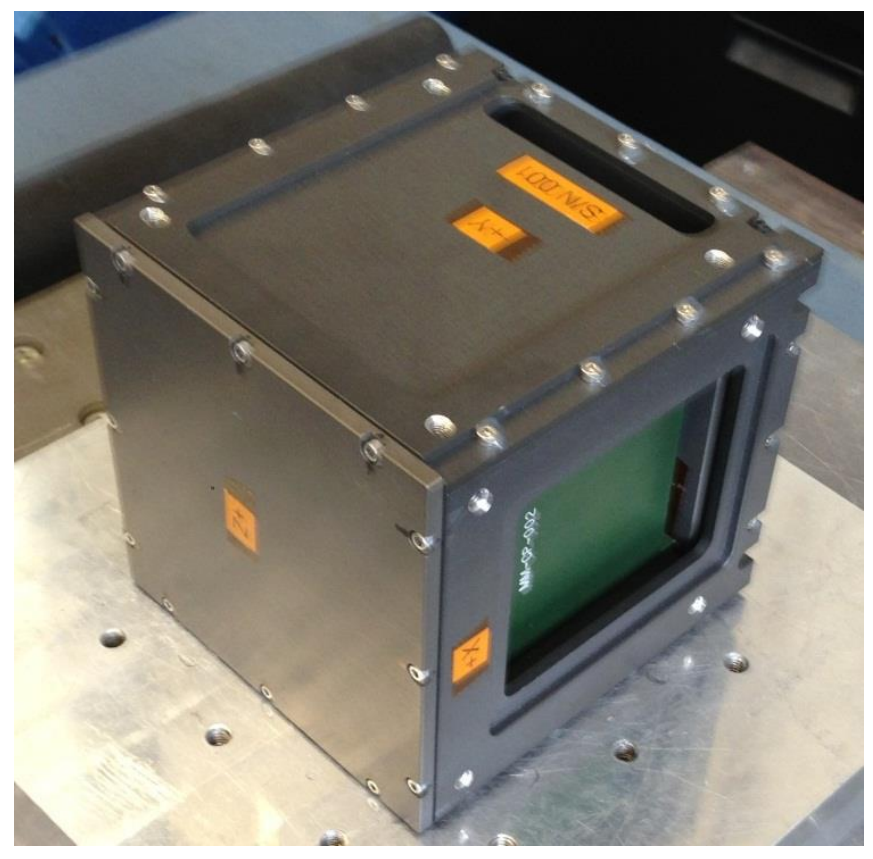

Figure 14: 1U TestPOD Mk III S/N 001.

\section{$3 U$ TestPOD Mk III}

The 3U TestPOD Mk III is the main test deployer used for CubeSats larger than 1U. It is slightly smaller than the P-POD, but with a stiffer and more robust design needed for repeated tests. Unlike the $1 \mathrm{U}$ TestPOD, the spring plungers are required to be staked after CubeSat integration. Figure 15 shows the 3U TestPOD Mk III S/N 014 used for vibration testing. 


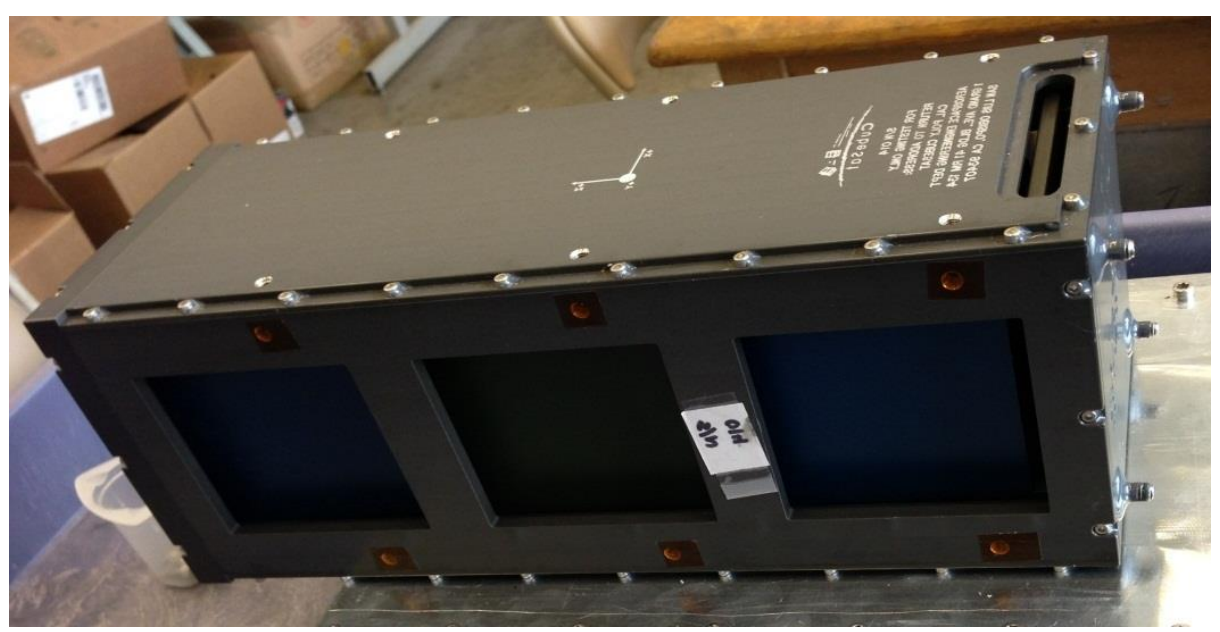

Figure 15: 3U TestPOD Mk III S/N 014.

\section{P-POD Mk. III Rev C}

The P-POD used in this thesis was the P-POD Mk. III Rev C. Unlike the TestPODs, P-PODs are flight hardware that deploy CubeSats on orbit. Over ten Rev C P-PODs have flown at the time of the writing of this thesis. P-PODs are design to be as light as possible, so strength margins are lower than the TestPOD's. This means the amount of testing is limited for the P-POD due to fatigue. Figure 16 shows the P-POD Mk. III Rev C S/N 44. 


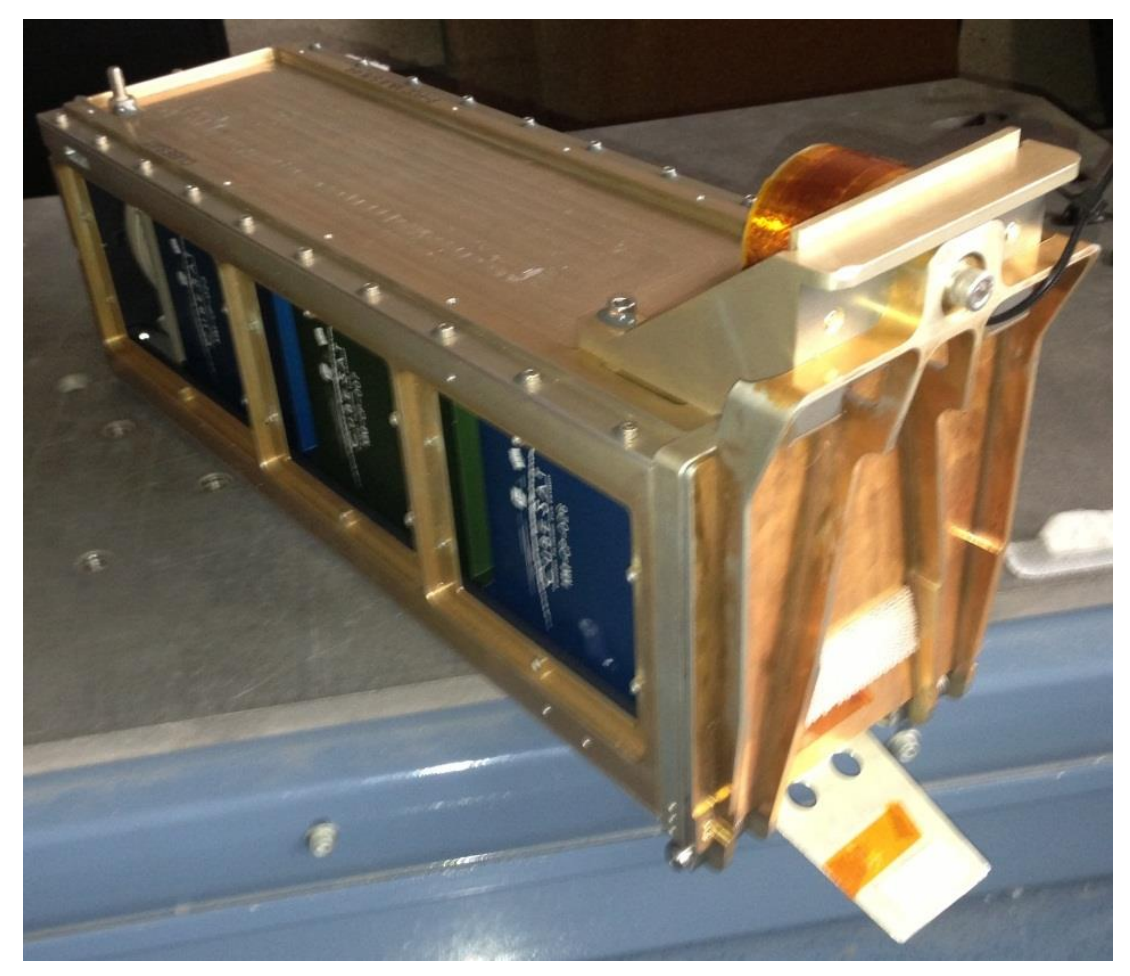

Figure 16: P-POD Mk. III Rev C S/N 44.

CubeSats

\section{CubeSat Mass Models}

CubeSat mass models were used to collect CubeSat response data inside the three deployers. Mass models are blocks of aluminum with dimensions that meet the CubeSat standard. Figure 17 shows a $1 \mathrm{~kg}$ CubeSat mass model. 


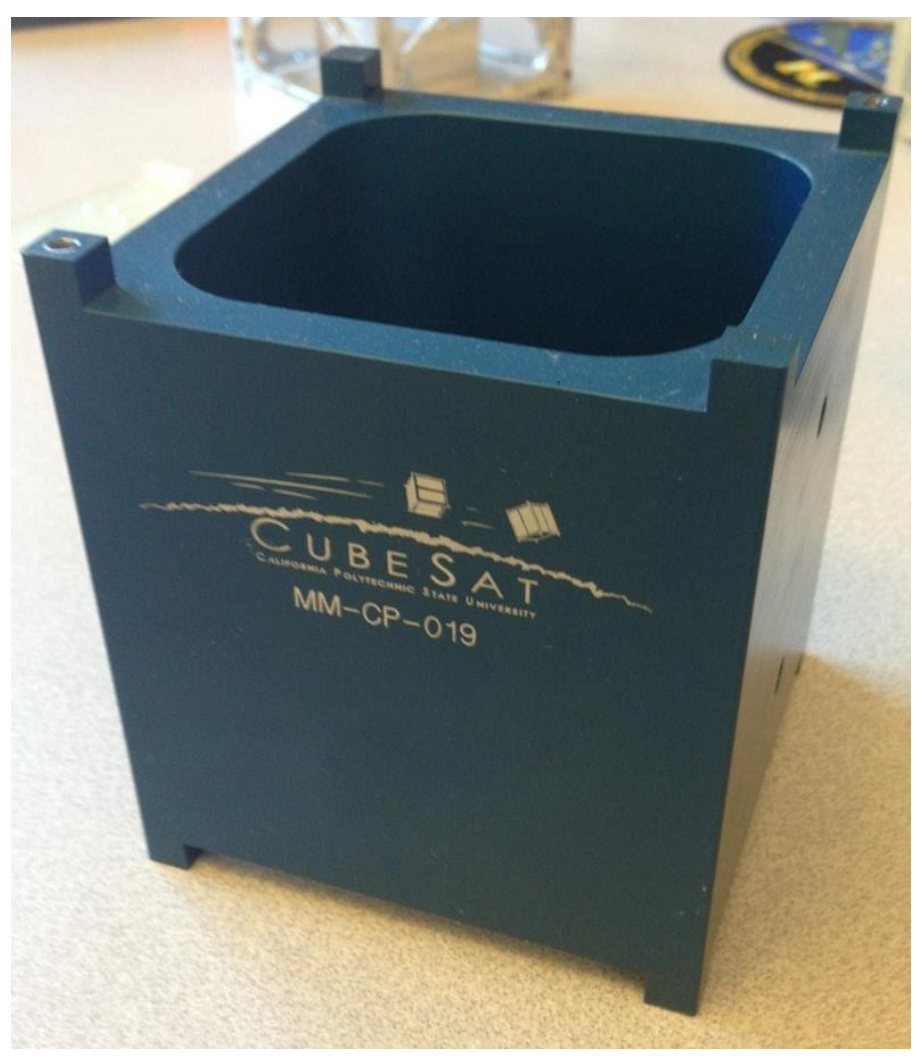

Figure 17: 1kg CubeSat mass model.

CubeSat mass models were used for testing because of their stiffness. FEA shows the first mode of free-free CubeSat mass models at $2160 \mathrm{~Hz}$. The first mode shape is shown in Figure 18. Further information about the FEA can be found in Appendix C. 


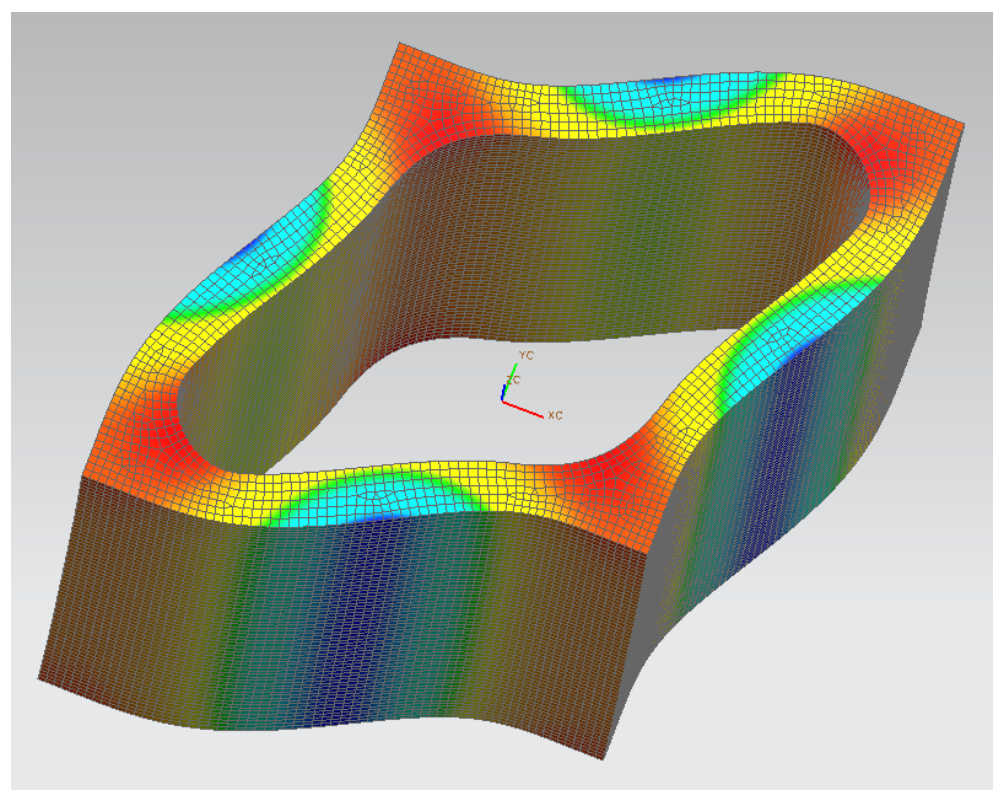

Figure 18: First mode shape of CubeSat mass model with free-free constraint at $2160 \mathrm{~Hz}$.

The CubeSat mass model can be modeled as a rigid body from $20-2000 \mathrm{~Hz}$ (the range of frequencies that will be tested). It is important to use a stiff CubeSat to ensure response data is from the input vibration and deployer, not from the CubeSat's dynamics. The use of CubeSat mass models provides an absolute baseline for CubeSat loads.

\section{Test CubeSat}

The Test CubeSat is a piece of extruded aluminum machined down to meet the CubeSat standard. Holes were drilled in the sides to attach aluminum plates with nuts and bolts to vary the mass. The walls of the Test CubeSat are much thinner than a mass model's walls, making the Test CubeSat a better representation of real CubeSat structures. The $\mathrm{X}$ axis of the Test CubeSat was machined down $1 / 8^{\text {th }}$ of an inch on each side so that rails on that axis could be removable. The Test CubeSat was primarily used 
in tests for Chapter 6 with soft rails, but some tests in chapter 3 were performed with standard rigid rails. Figure 19 shows the Test CubeSat.

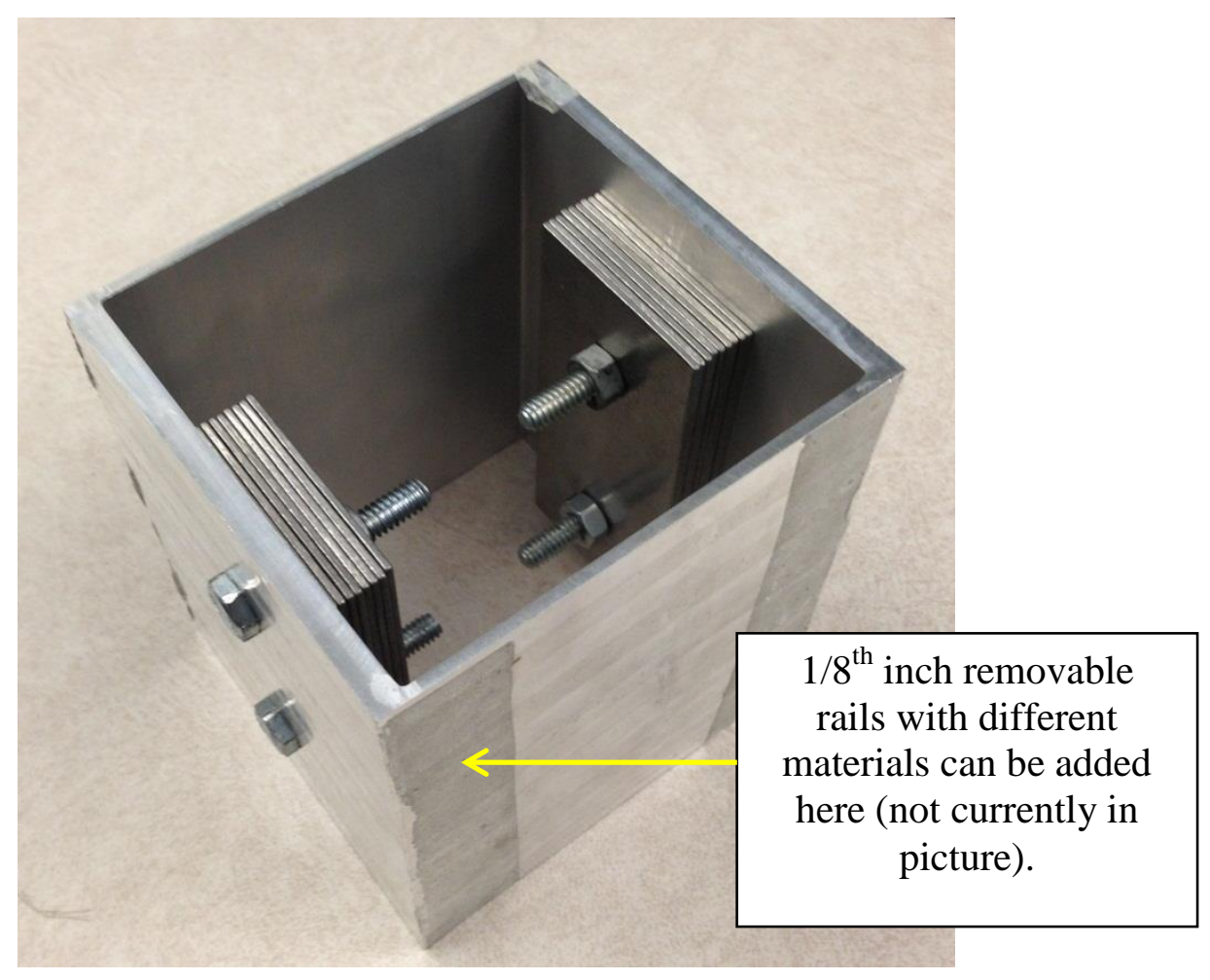

Figure 19: Test CubeSat.

As mentioned earlier, the Test CubeSat has thinner walls than the CubeSat mass model. FEA shows that the Test CubeSat has many resonant peaks less than 2,000 Hz. This means that some peaks from accelerometer response data on the Test CubeSat will be from the CubeSat's dynamics and not from the random vibration profile or deployer. Figure 20 shows the third mode of the Test CubeSat, a mode with a large amount of mass participation where the accelerometer is mounted. Further information about the FEA is shown in Appendix C. 


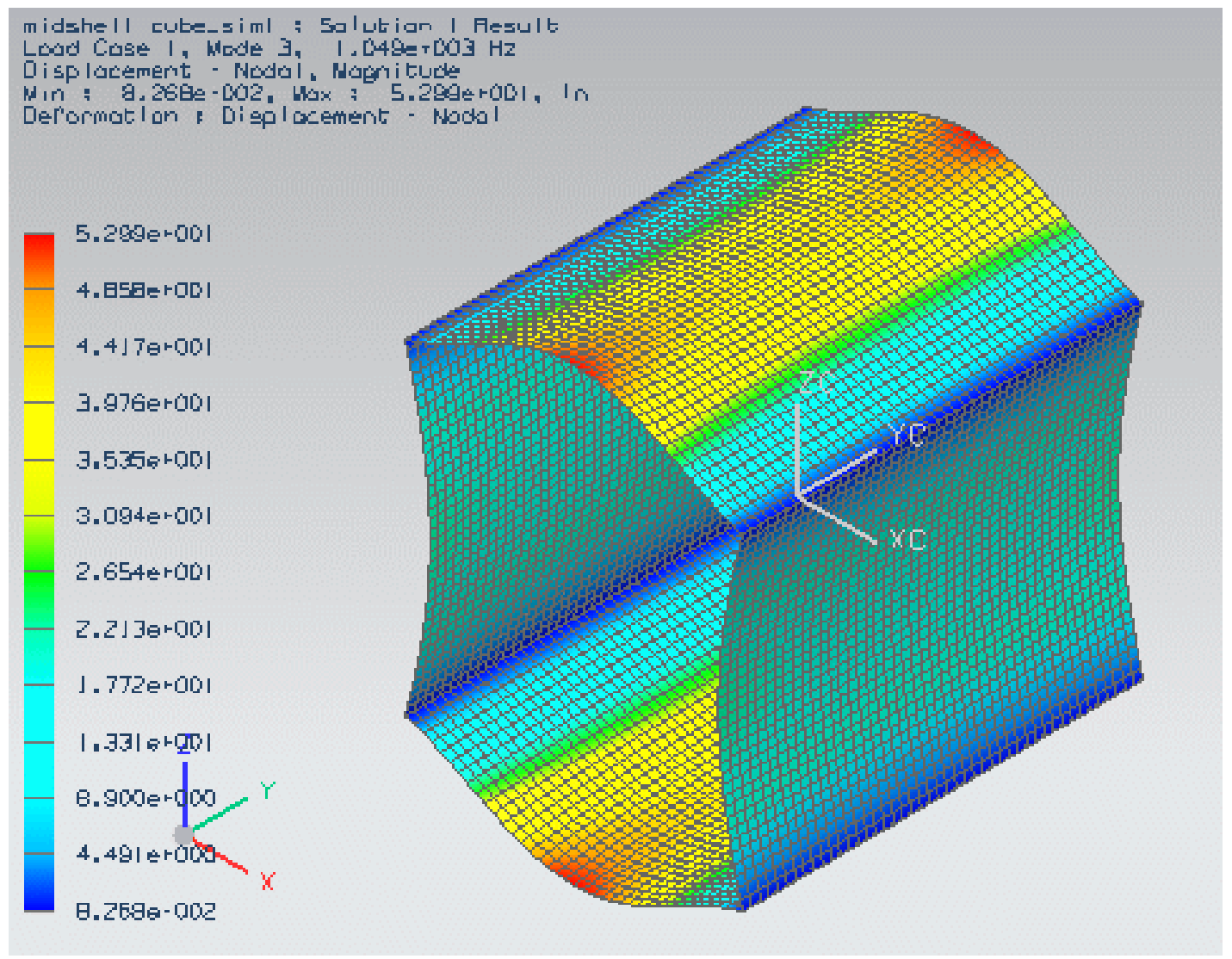

Figure 20: Third mode of Test CubeSat $(1049 \mathrm{~Hz})$.

\section{Vibration Equipment}

\section{Interface Plates}

Two sets of interface plates are used to attach deployers to the vibration table.

Characterization of these interface plates was not done. It is assumed in this thesis the interface plates are an extension of the deployer's structure. This assumption is made because it is not the goal of this thesis to characterize what causes the peaks in the deployers, but how those peaks effect CubeSat loads. 
There is a single interface plate used to attach the $1 \mathrm{U}$ TestPOD to the vibration table. Figure 21 shows the $1 \mathrm{U}$ TestPOD on the $1 \mathrm{U}$ interface plate.

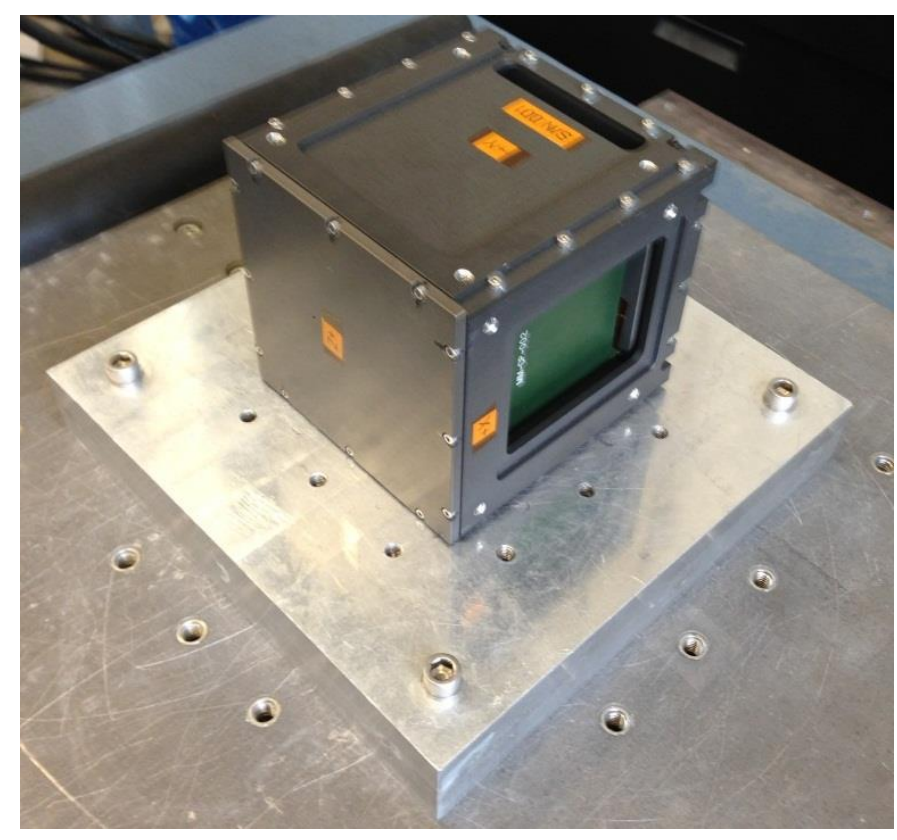

Figure 21: 1U TestPOD on 1U interface plate.

The $3 \mathrm{U}$ TestPOD and P-POD use two interface plates. A thick plate is bolted to the table, and a thinner plate is attached to the deployer. Figure 22 shows the $3 \mathrm{U}$ TestPOD attached to the vibration table using the two $3 \mathrm{U}$ interface plates. The P-POD utilizes the same setup. 


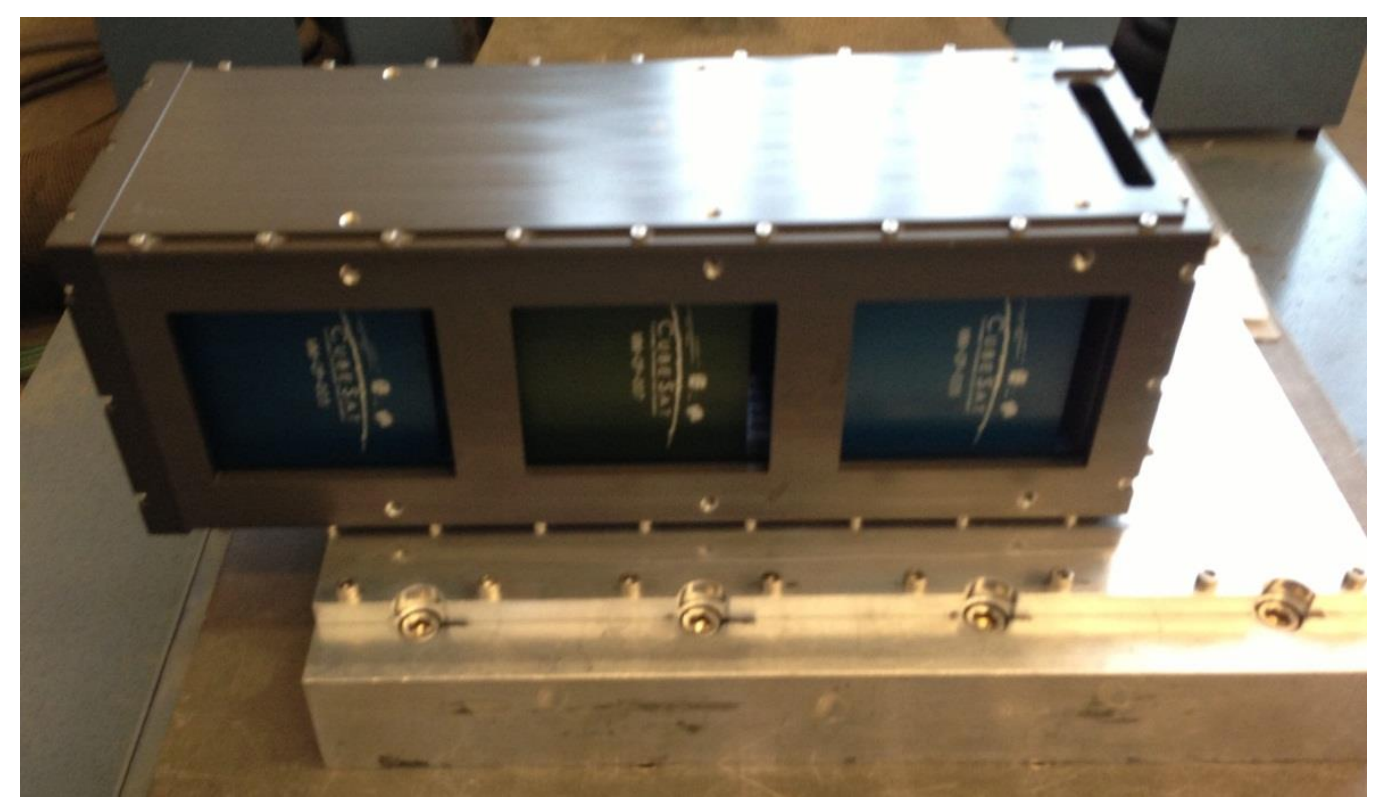

Figure 22: 3U TestPOD attached to table with two interface plates.

\section{Vibration Table}

The Unholtz Dickie vibration table in the Cal Poly Aerospace Composites and Structures Lab was used for vibration testing. The table can rotate from a slip position to a vertical position so all three axes of testing could be completed using the two interface plates shown before. Figure 23 shows the vibration table in the slip position and Figure 24 shows the vibration table amplifier. 


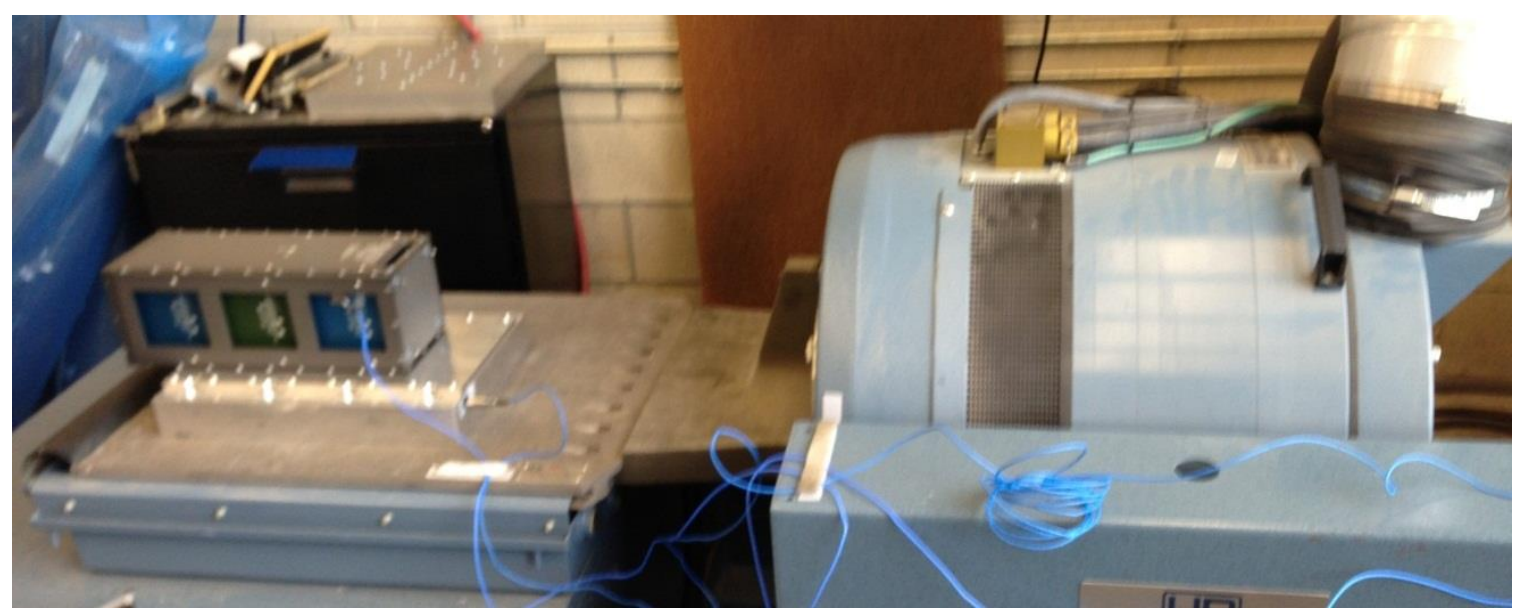

Figure 23: Vibration table.

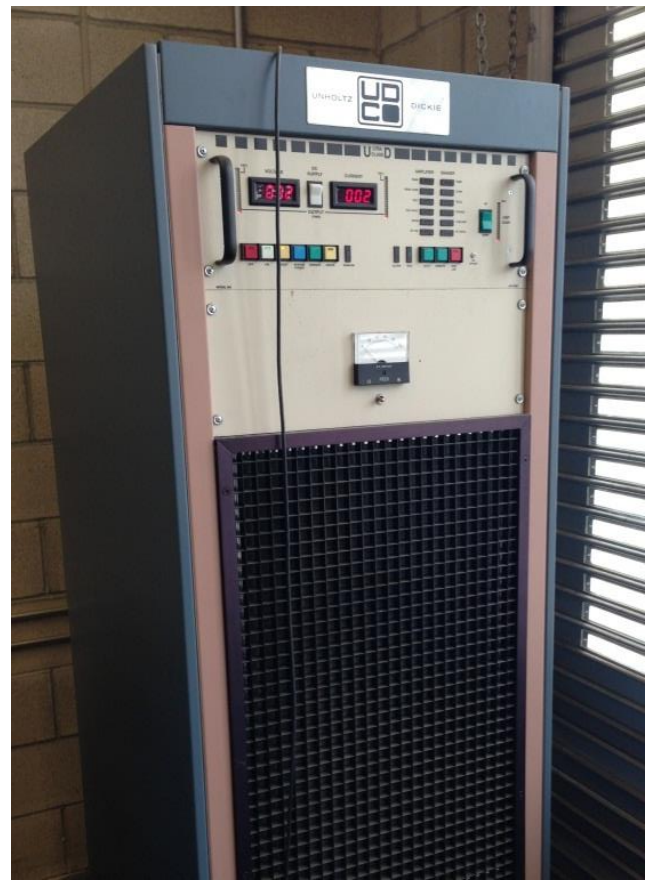

Figure 24: Vibration table amplifier.

\section{Accelerometers}

Two accelerometers were used during vibration testing: one single axis

accelerometer used for control and one three axis accelerometer used for response. The 
single axis control accelerometer was from PCB, model number 353B04. Its last calibration date was $1 / 25 / 06$. The three axis response accelerometer was also from PCB, model number $354 \mathrm{C} 10$. It was last calibrated on $7 / 6 / 11$. Figure 25 shows the single axis accelerometer and Figure 26 shows the three axis accelerometer.

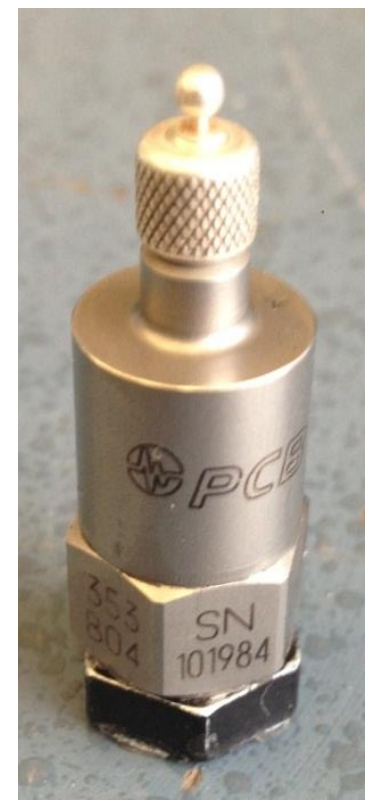

Figure 25: Single axis control accelerometer (PCB M/N 353B04).

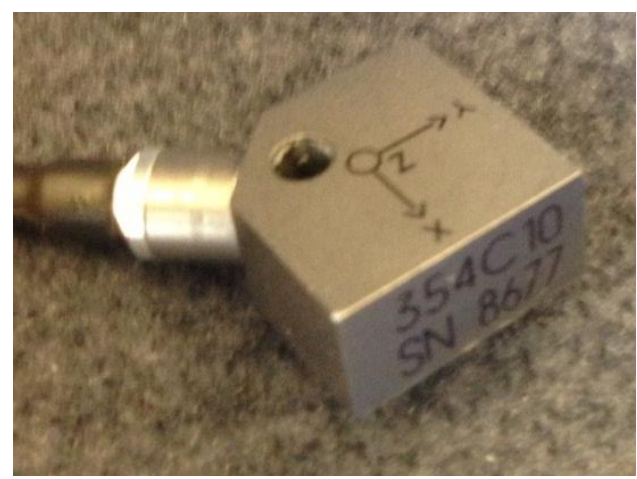

Figure 26: Three axis response accelerometer (PCB M/N 354C10). 
The accelerometers were attached to test articles using scotch tape and super glue. Scotch tape was placed on the test article, and the accelerometer was then super glued on top of the scotch tape. This method was used instead of hard mounting the accelerometer with a screw for ease of varying accelerometer location. Figure 27 shows an example of the response accelerometer mounted to a test article using the method described above.

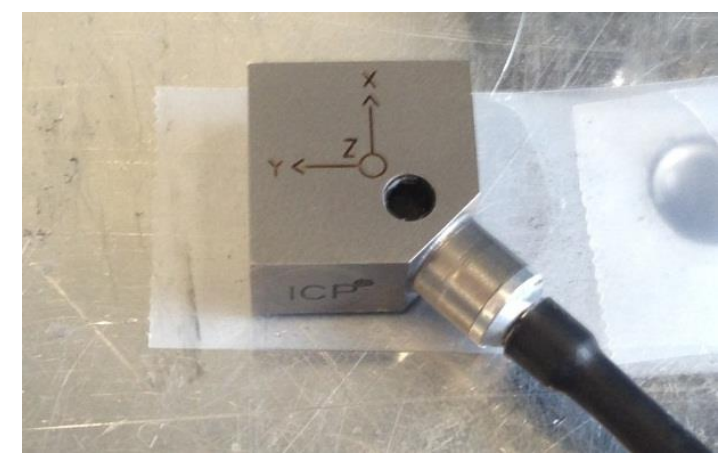

Figure 27: Example of accelerometer attachment to test article.

\section{Data Acquisition System}

The four channels from the accelerometers (one from the single axis control accelerometer, three from the three axis response accelerometer) are routed through an analog to digital converter to Vwin II software on a PC. Vwin II software is used to operate the vibration table, plot the data, and store the raw data as .csv files.

\section{Vibration Testing}

\section{Vibration Test Profiles}

Sine sweep and random vibration tests were the two types of tests run during this thesis. Sine sweep tests were primarily used to obtain information about the deployer's resonant peaks. Random vibration tests where primarily used for analysis of CubeSat 
loads and the effect the deployer has on them. Because CubeSats response is not proportional to vibration input, random vibration tests were mostly used for analysis, as their levels are closer to the loads CubeSats would see during launch.

\section{Sine Sweep Tests}

Sine sweep tests were mostly performed on the deployer to obtain information about the deployer's resonant peaks and frequency response. Sine sweep tests with the response accelerometer on the CubeSats were also performed, but to a much lesser extent. This is because the CubeSat response changes as loads increase, so the low level sine sweep data for CubeSats is not as relevant as high level random vibration tests.

Sine sweeps were run from 20 to $2,000 \mathrm{~Hz}$ at $0.55 \mathrm{~g}$ at a sweep rate of three octaves per minute. Figure 28 shows the input for sine sweep tests.

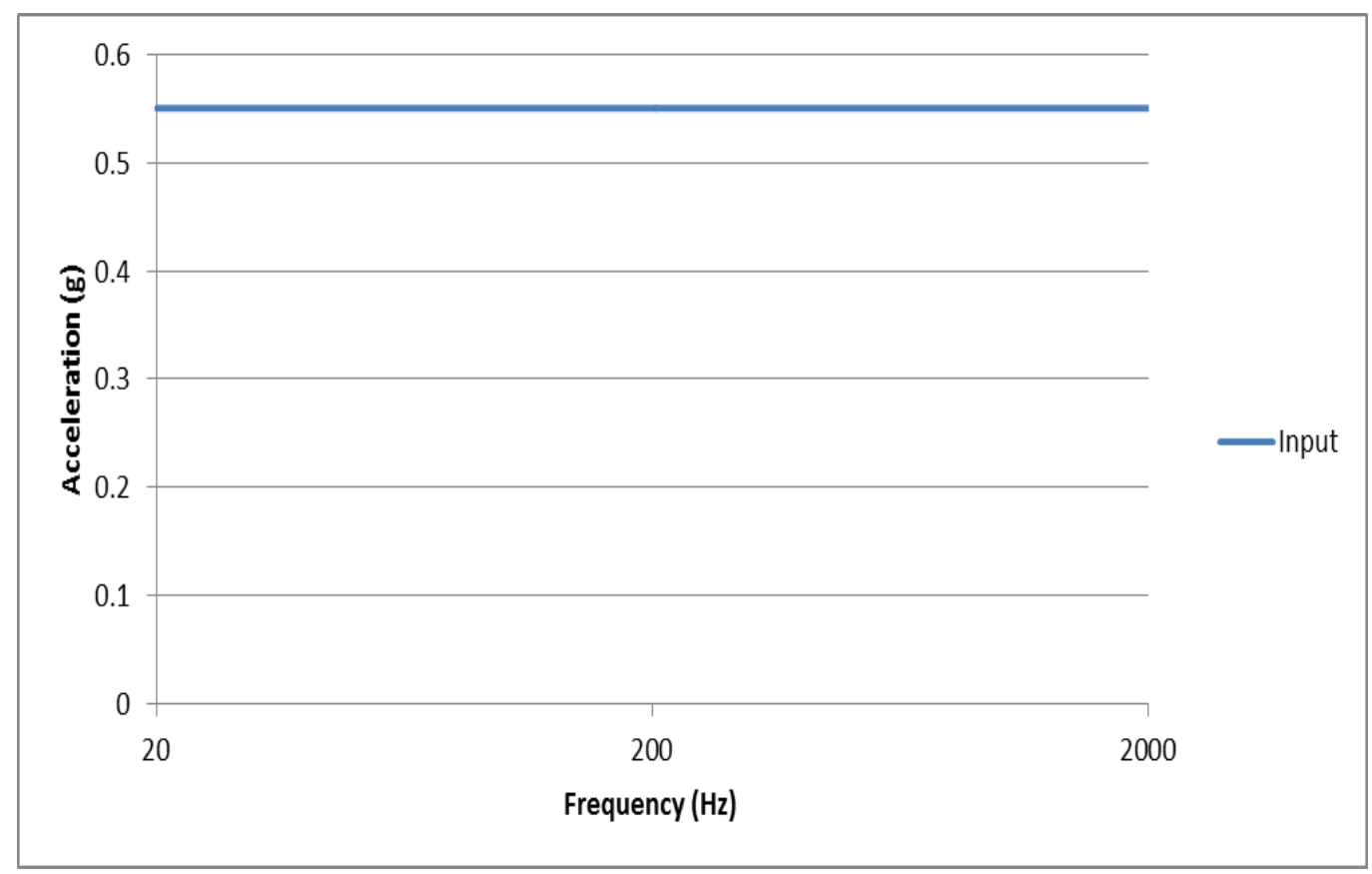

Figure 28: Input for sine sweep tests. 


\section{Random Vibration Tests}

For vibration data to be a good representation of how CubeSats respond in deployers, the input magnitude and frequency band must be similar to what is seen during testing and launch (this is especially true with non-linear systems like the CubeSatdeployer system). For this reason, random vibration tests were the main tests run for analysis of the CubeSat-deployer system.

The GEVS acceptance profile was the primary random vibration profile used during testing. GEVS is useful because it is a public random vibration profile that is familiar to many people in the CubeSat community. Acceptance levels were chosen to obtain flight level loads $\left(9.99 \mathrm{G}_{\mathrm{rms}}\right)$ without over testing the deployers by using qualifying levels ( $14 \mathrm{G}_{\mathrm{rms}}$ or higher). Figure 29 shows the GEVS random vibration profile.

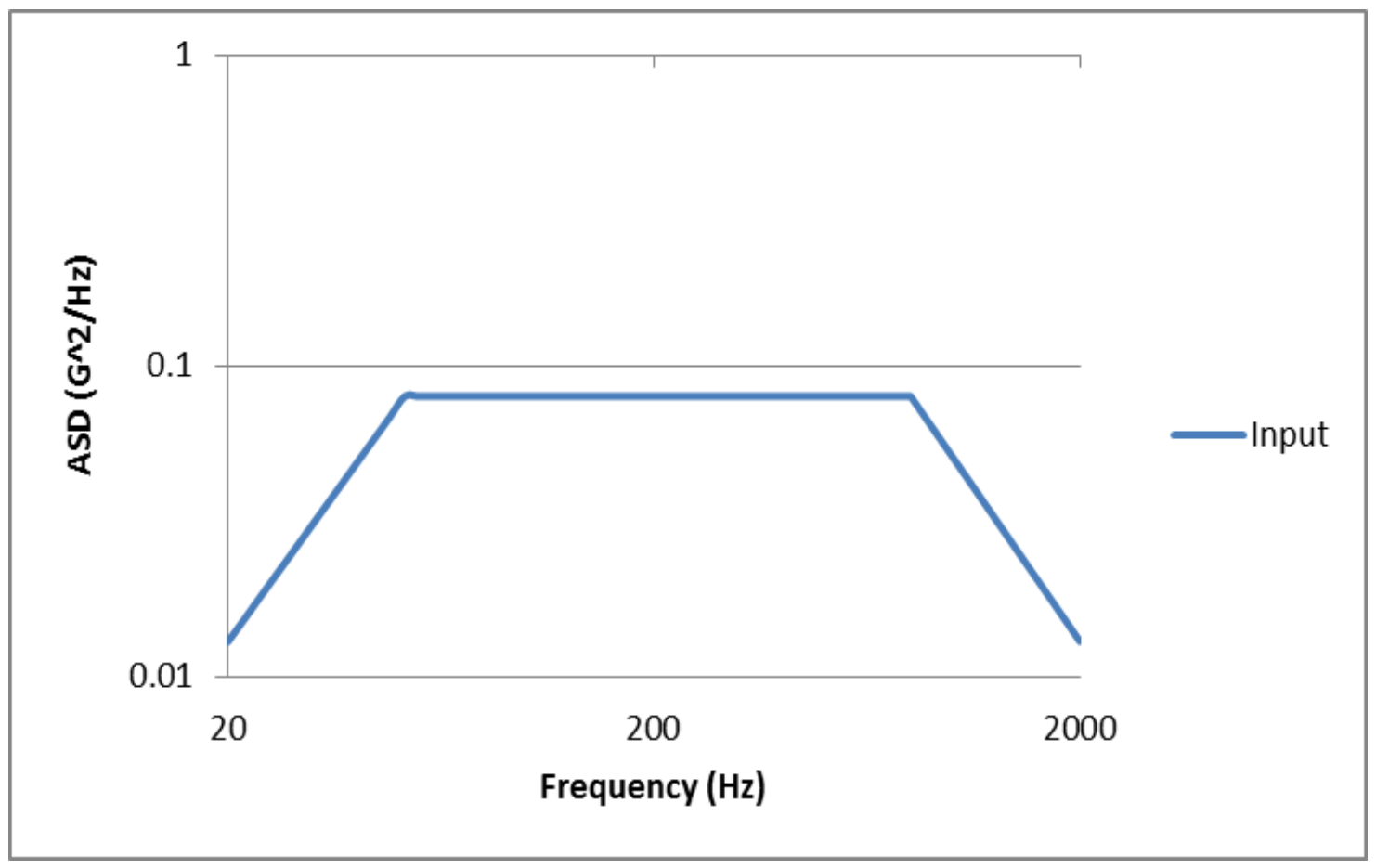

Figure 29: GEVS acceptance random vibration profile $\left(9.99 \mathrm{G}_{\mathrm{rms}}\right)$. 
Another random vibration profile was used for comparison purposes. This profile was created for use in this thesis only, but is based off previous random vibration profiles. Figure 30 shows random vibration profile number 1.

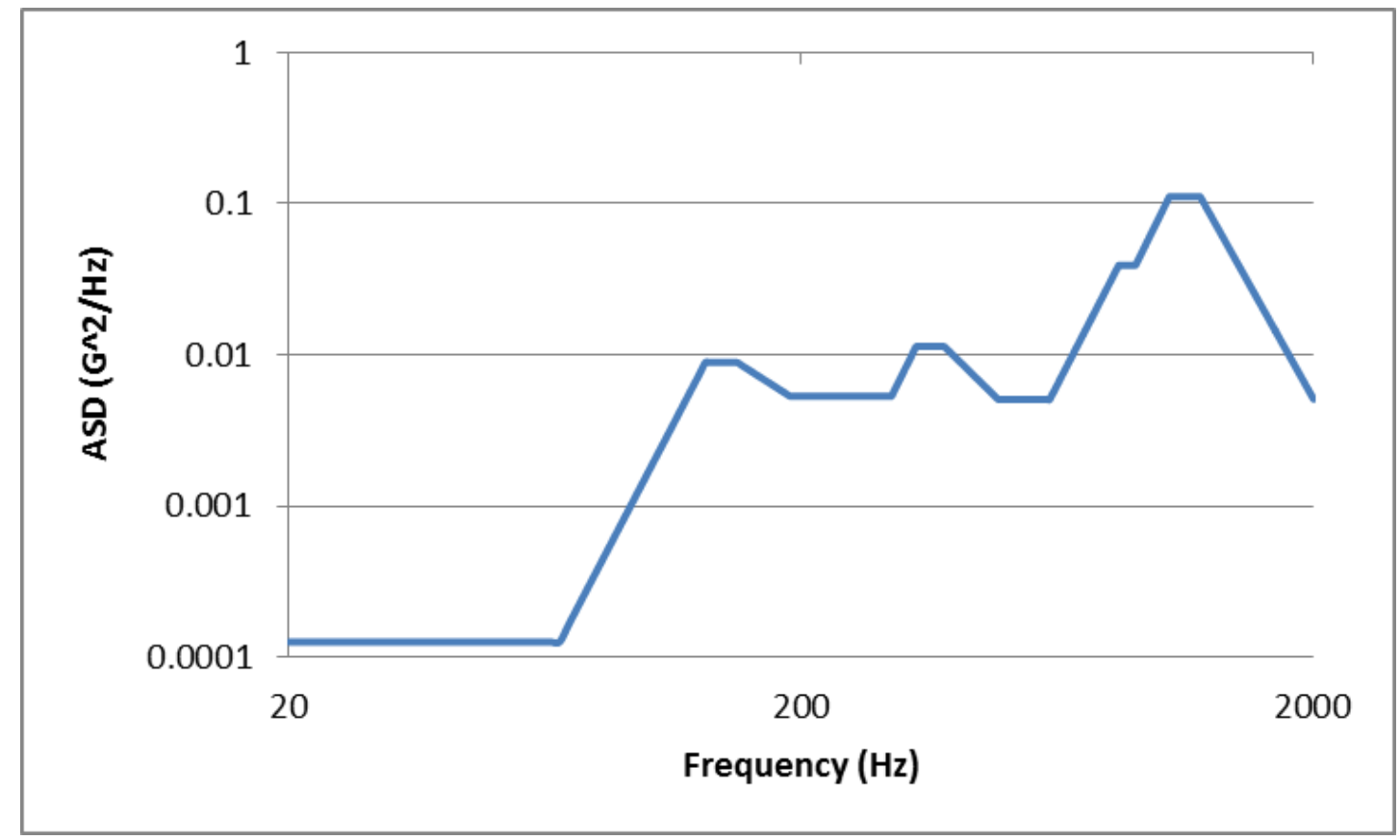

Figure 30: Random vibration profile 1.

Vibration Tests

\section{Accelerometer Verification Tests}

The control accelerometer was calibrated on $1 / 25 / 06$, and the three axis accelerometer was calibrated on $7 / 6 / 11$. Sine sweeps were performed with both accelerometers mounted on near-rigid test articles to ensure they both have the same response. The $1 \mathrm{U}$ and $3 \mathrm{U}$ interface plates were used as the near-rigid test articles. These tests also verify the accelerometer attachment method does not add additional dynamics to the response data. Figure 31, Figure 33, and Figure 35 show the accelerometer 
placement for the $\mathrm{X}, \mathrm{Y}$ and $\mathrm{Z}$ axis verification tests. Figure 32, Figure 34, Figure 36 show the raw data.

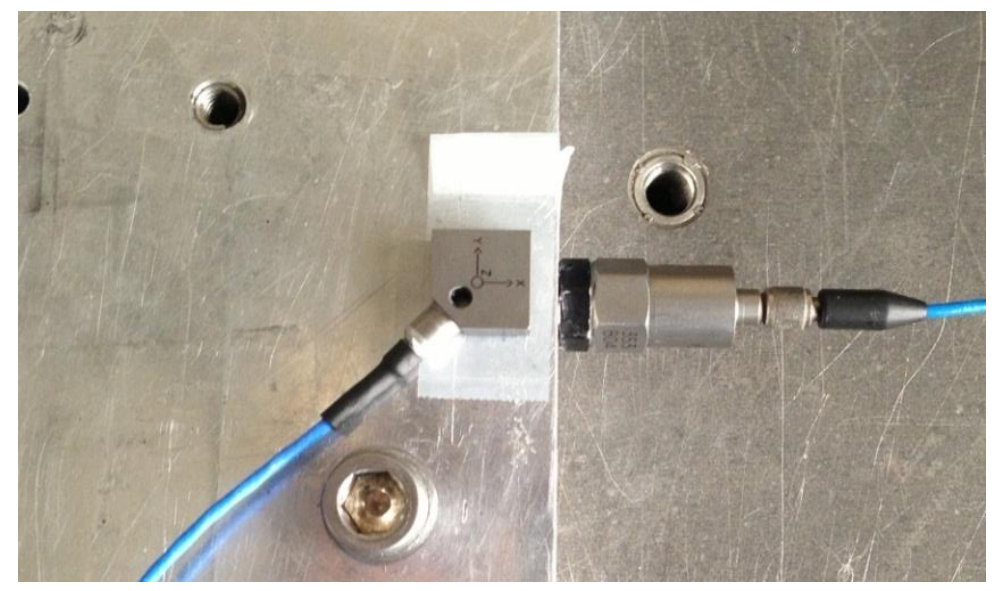

Figure 31: Accelerometer placement on $1 \mathrm{U}$ interface plate for $\mathrm{X}$ axis verification.
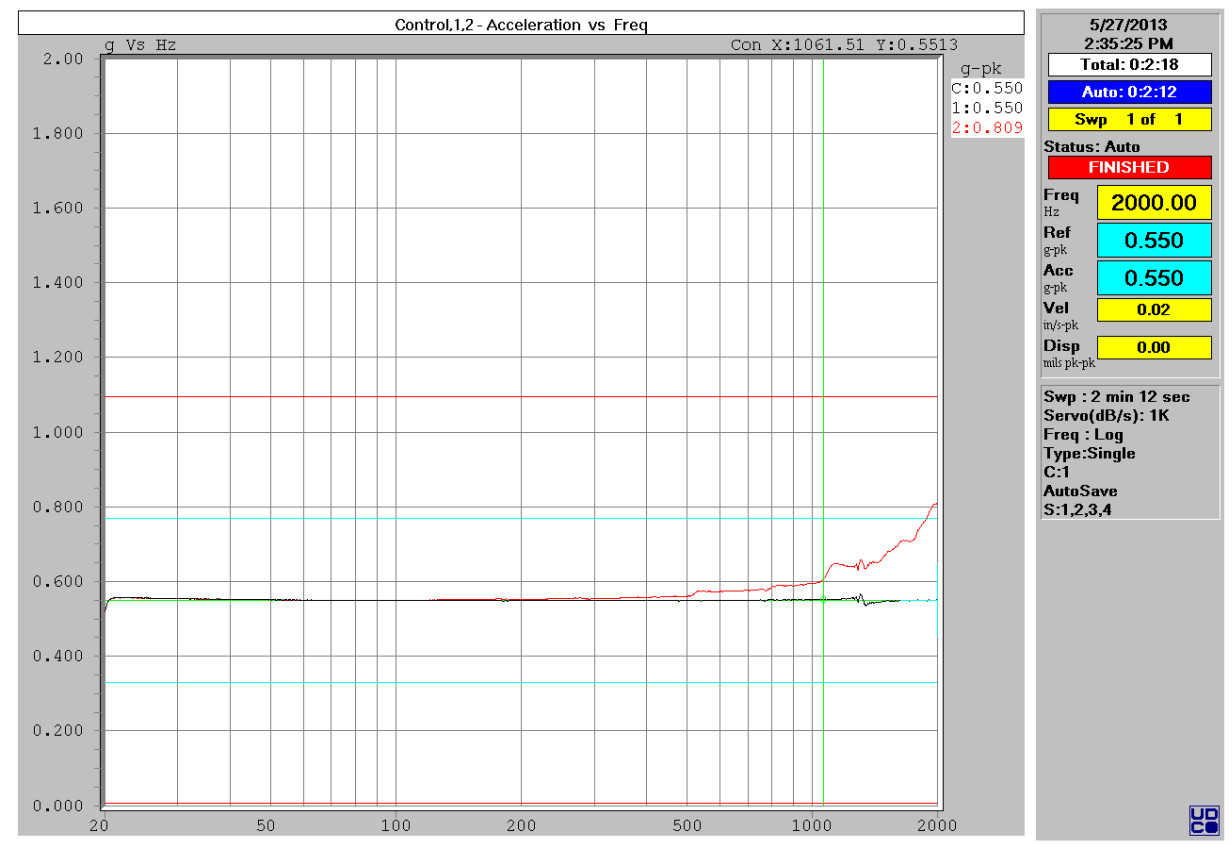

Figure 32: $\mathrm{X}$ axis verification sine sweep. 


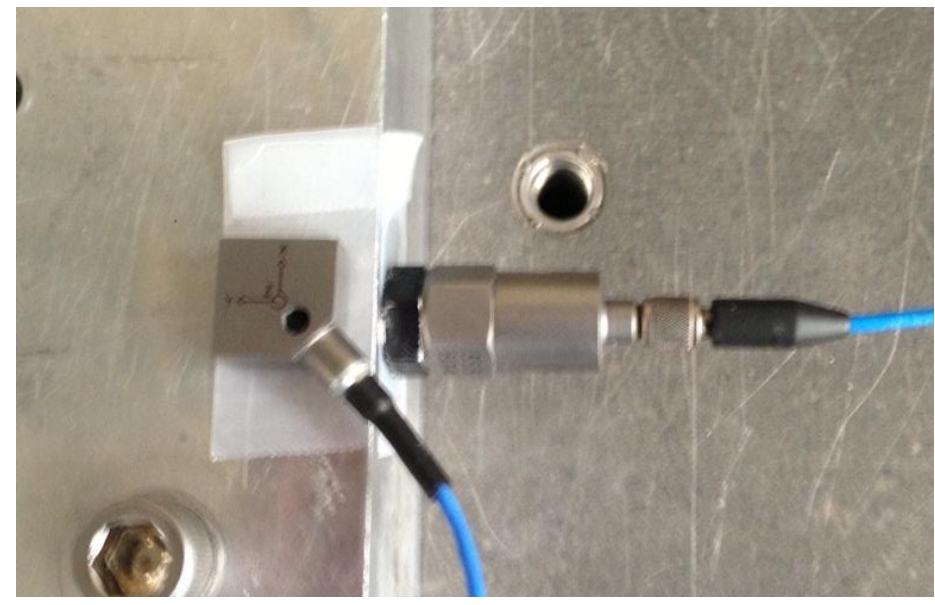

Figure 33: Accelerometer placement on $1 \mathrm{U}$ interface plate for $\mathrm{Y}$ axis verification.
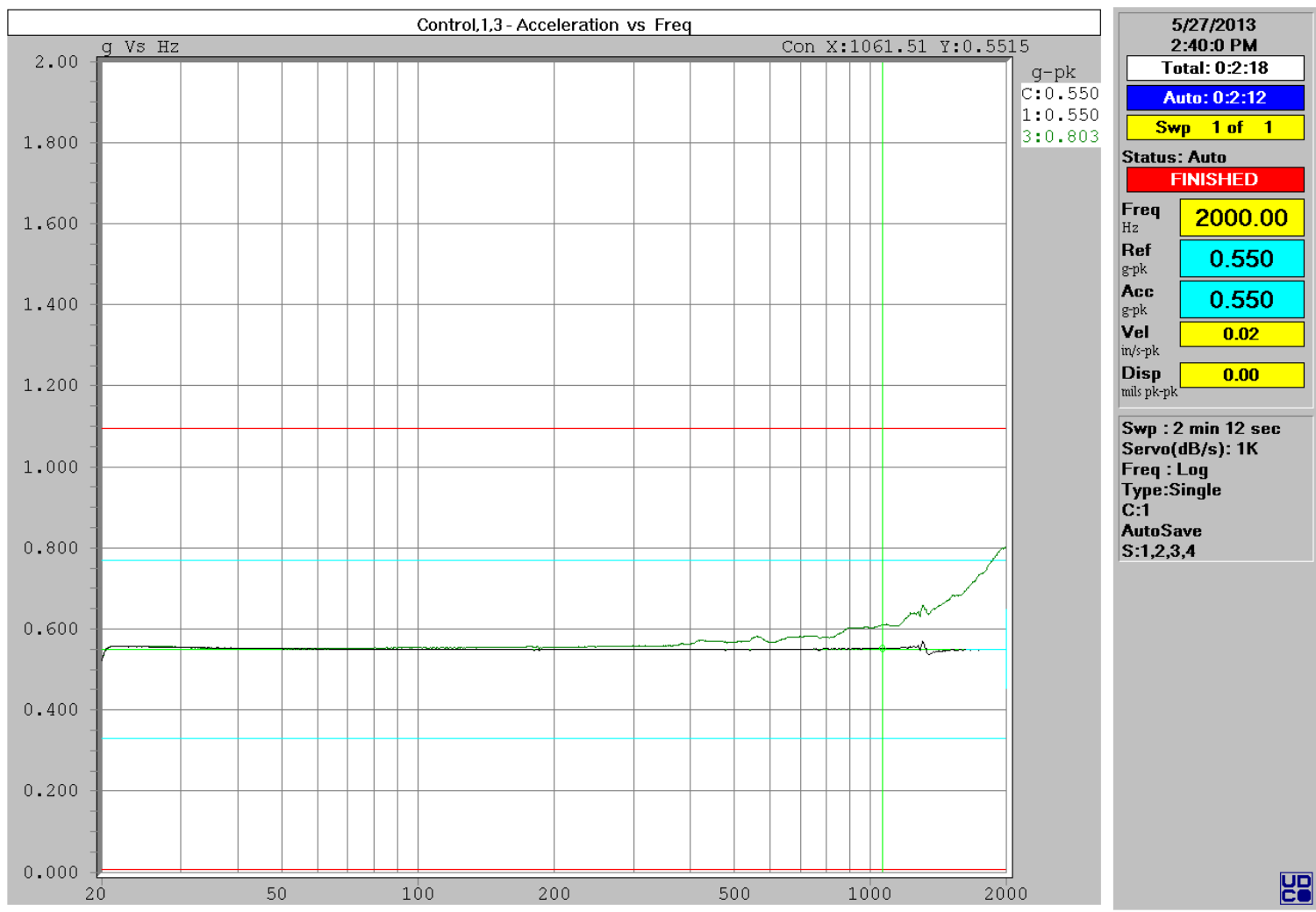

Figure 34: $Y$ axis calibration

Page 39 


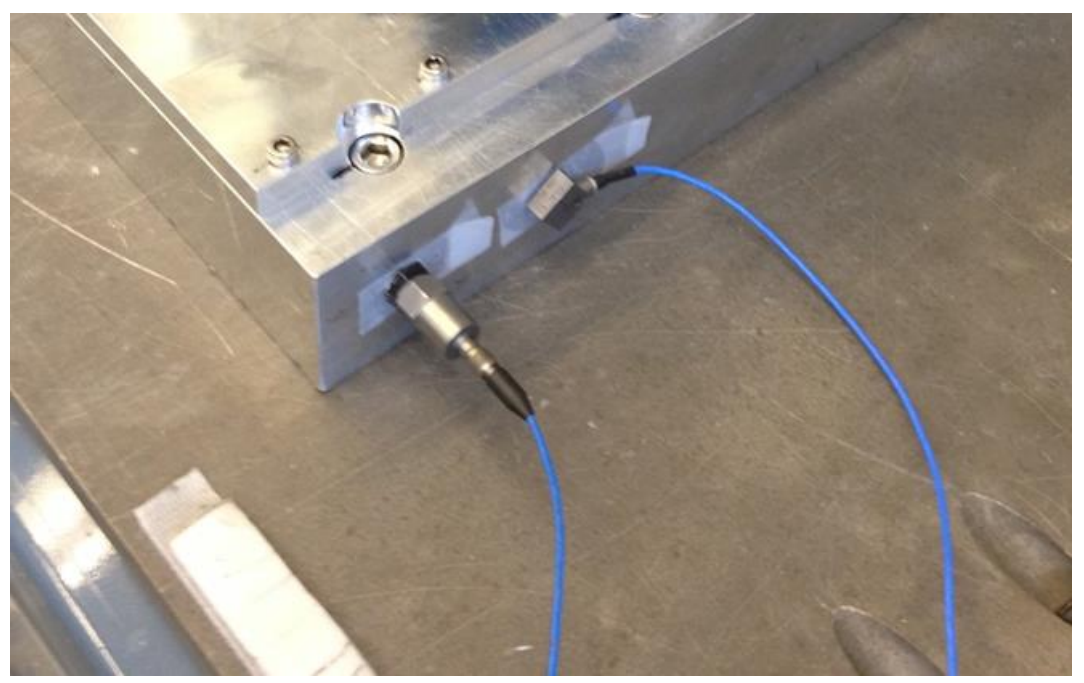

Figure 35: Accelerometer placement on $3 \mathrm{U}$ interface plate for $\mathrm{Z}$ axis verification.
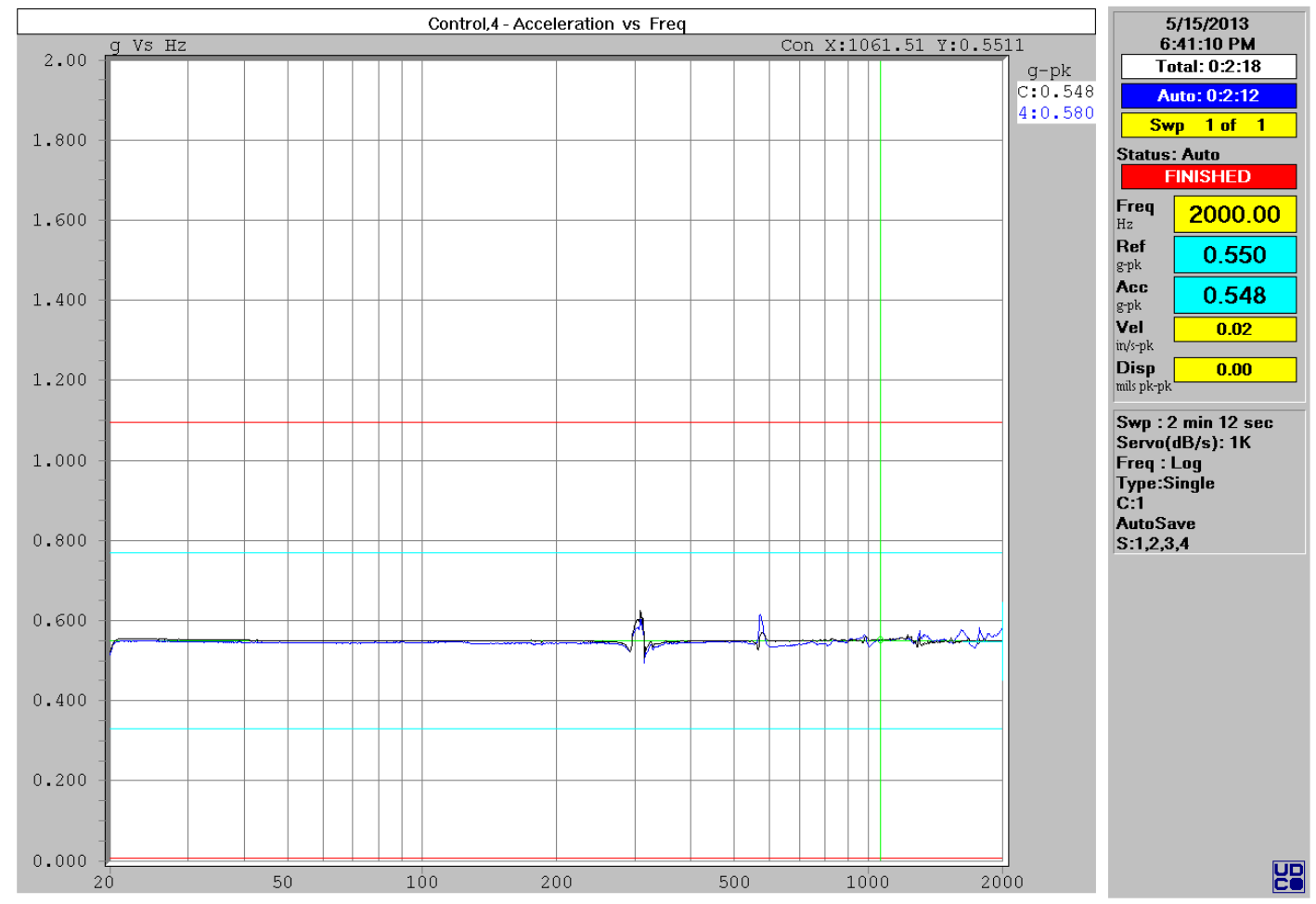

Figure 36: $\mathrm{Z}$ axis calibration. 
The $\mathrm{Z}$ axis of the three axis accelerometer responded almost exactly with the single axis accelerometer. The $\mathrm{X}$ and $\mathrm{Y}$ axes of the three axis accelerometer responded with the single axis accelerometer in the lower frequencies, but then started to diverge in the higher frequencies.

\section{CubeSat and Deployer Testing}

The goal of vibration testing was to obtain data to create a model for the CubeSatdeployer system. Tests were performed in the $\mathrm{X}$ and $\mathrm{Z}$ axes of the $1 \mathrm{U}$ TestPOD and $3 \mathrm{U}$ TestPOD, and in all three axes for the P-POD. Sine sweeps were run with the response accelerometer on the deployer to obtain the deployer's resonance peaks. Random vibration tests were performed with response accelerometer on the CubeSat and deployer. Some random vibration tests on CubeSats were repeated with the accelerometer mounted in different locations to understand how loads change by location in the deployer. Table 1 provides a summary of every test that was performed. The pictures and raw data are shown in Appendix D. Discussion of the data is shown in Chapter 4. 
Table 1: Summary of Vibration Tests Performed.

\begin{tabular}{|c|c|c|c|c|c|}
\hline $\begin{array}{c}\text { Test } \\
\#\end{array}$ & Deployer & Axis & $\begin{array}{c}\text { Response } \\
\text { Accel. } \\
\text { Location }\end{array}$ & Profile & Spring Plungers \\
\hline 1 & 1U TestPOD & $\mathrm{Z}$ & Deployer & Sine & Standard Integration \\
\hline 2 & 1U TestPOD & $\mathrm{Z}$ & Deployer & Random & Standard Integration \\
\hline 3 & $1 \mathrm{U}$ TestPOD & $\mathrm{Z}$ & CubeSat & Random & Standard Integration \\
\hline 4 & 1U TestPOD & $\mathrm{Z}$ & CubeSat & Random & Bolts: Backed Out 1.5 Turns \\
\hline 5 & 1U TestPOD & $X$ & Deployer & Sine & Standard Integration \\
\hline 6 & 1U TestPOD & $X$ & Deployer & Random & Standard Integration \\
\hline 7 & 1U TestPOD & $\mathrm{X}$ & CubeSat & Random & Standard Integration \\
\hline $8-1$ & 1U TestPOD & $\mathrm{X}$ & CubeSat & Random & Dialed in Tight \\
\hline $8-2$ & 1U TestPOD & $X$ & CubeSat & Sine & Dialed in Tight \\
\hline $9-1$ & $1 \mathrm{U}$ TestPOD & $\mathrm{X}$ & CubeSat & Random & Backed Out One Turn \\
\hline $9-2$ & 1U TestPOD & $\mathrm{X}$ & CubeSat & Sine & Backed Out One Turn \\
\hline 10 & 3U TestPOD & $\mathrm{Z}$ & Deployer & Sine & Standard Integration \\
\hline 11 & 3U TestPOD & $\mathrm{Z}$ & Deployer & Random & Standard Integration \\
\hline $12-1$ & 3U TestPOD & $\mathrm{Z}$ & CubeSat & Random & Standard Integration \\
\hline $12-2$ & 3U TestPOD & $\mathrm{Z}$ & CubeSat & Random & Standard Integration \\
\hline 13 & 3U TestPOD & $X$ & Deployer & Sine & Standard Integration \\
\hline 14 & 3U TestPOD & $\mathrm{X}$ & Deployer & Random & Standard Integration \\
\hline $15-1$ & 3U TestPOD & $\mathrm{X}$ & CubeSat & Random & Standard Integration \\
\hline $15-2$ & 3U TestPOD & $X$ & CubeSat & Random & Standard Integration \\
\hline 16 & P-POD & $\mathrm{Z}$ & Deployer & Sine & Standard Integration \\
\hline 17 & P-POD & $\mathrm{Z}$ & Deployer & Random & Standard Integration \\
\hline $18-1$ & P-POD & $\mathrm{Z}$ & CubeSat & Random & Standard Integration \\
\hline $18-2$ & P-POD & $\mathrm{Z}$ & CubeSat & Random & Standard Integration \\
\hline 19 & P-POD & $\mathrm{Z}$ & CubeSat & Random 1 & Standard Integration \\
\hline 20 & P-POD & $\mathrm{X}$ & Deployer & Sine & Standard Integration \\
\hline 21 & P-POD & $X$ & Deployer & Random & Standard Integration \\
\hline $22-1$ & P-POD & $\mathrm{X}$ & CubeSat & Random & Standard Integration \\
\hline $22-2$ & P-POD & $X$ & CubeSat & Random & Standard Integration \\
\hline 23 & P-POD & $X$ & CubeSat & Random 1 & Standard Integration \\
\hline 24 & P-POD & $\mathrm{Y}$ & Deployer & Sine & Standard Integration \\
\hline 25 & P-POD & $\mathrm{Y}$ & Deployer & Random & Standard Integration \\
\hline $26-1$ & P-POD & $Y$ & CubeSat & Random & Standard Integration \\
\hline $26-2$ & P-POD & $\mathrm{Y}$ & CubeSat & Random & Standard Integration \\
\hline 27 & P-POD & Y & CubeSat & Random 1 & Standard Integration \\
\hline
\end{tabular}




\section{CHAPTER 4: Modeling the CubeSat-Deployer System}

In the previous Chapter, over 30 vibration tests were run to obtain data to model the CubeSat-deployer system. In this chapter, that data will be used to analyze the CubeSat constraint, develop a vibration model, and understand how the deployer's design affects CubeSat vibration loads.

\section{The CubeSat Constraint in the Deployer}

\section{Z Axis Constraint}

The deployer's spring plungers completely constrain the CubeSat in the Z axis. Because of this, CubeSats can be modeled as fixed inside the deployer in the $\mathrm{Z}$ axis. This is illustrated in Figure 37, where the vibration data shows the CubeSat responding with the $1 \mathrm{U}$ TestPOD's resonance. 


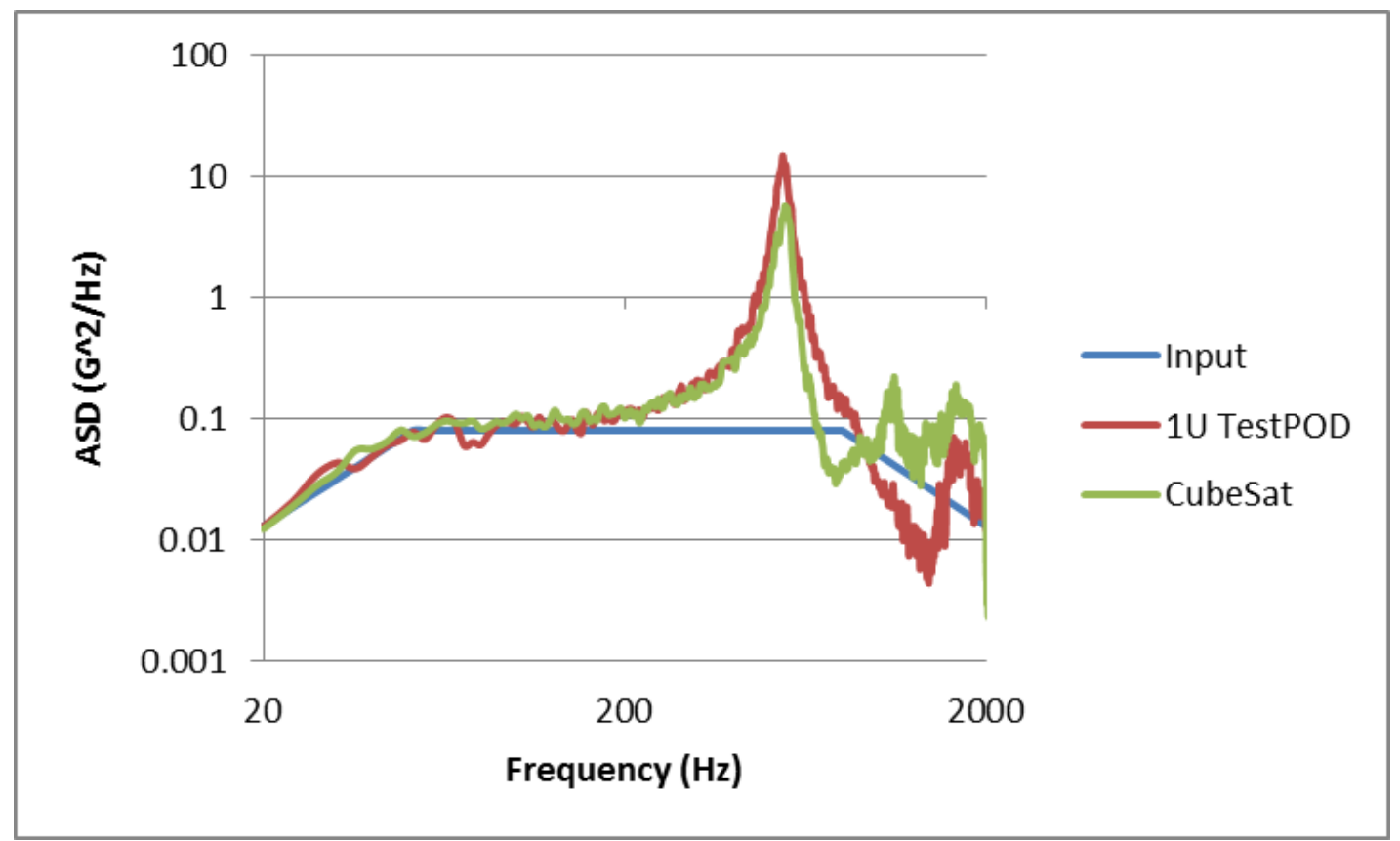

Figure 37: $\mathrm{Z}$ axis vibration data of a $1 \mathrm{~kg}$ CubeSat mass model in a $1 \mathrm{U}$ TestPOD (test numbers 2 and

3).

The similarity of the frequency responses of both the CubeSat and 1U TestPOD show that the CubeSat is fixed inside the deployer.

The same result is seen in the $\mathrm{Z}$ axis of the $3 \mathrm{U}$ TestPOD and P-POD. Figure 38 and Figure 39 show $\mathrm{Z}$ axis vibration data for three $1 \mathrm{~kg}$ CubeSat mass models integrated into a $3 \mathrm{U}$ TestPOD and a P-POD. 


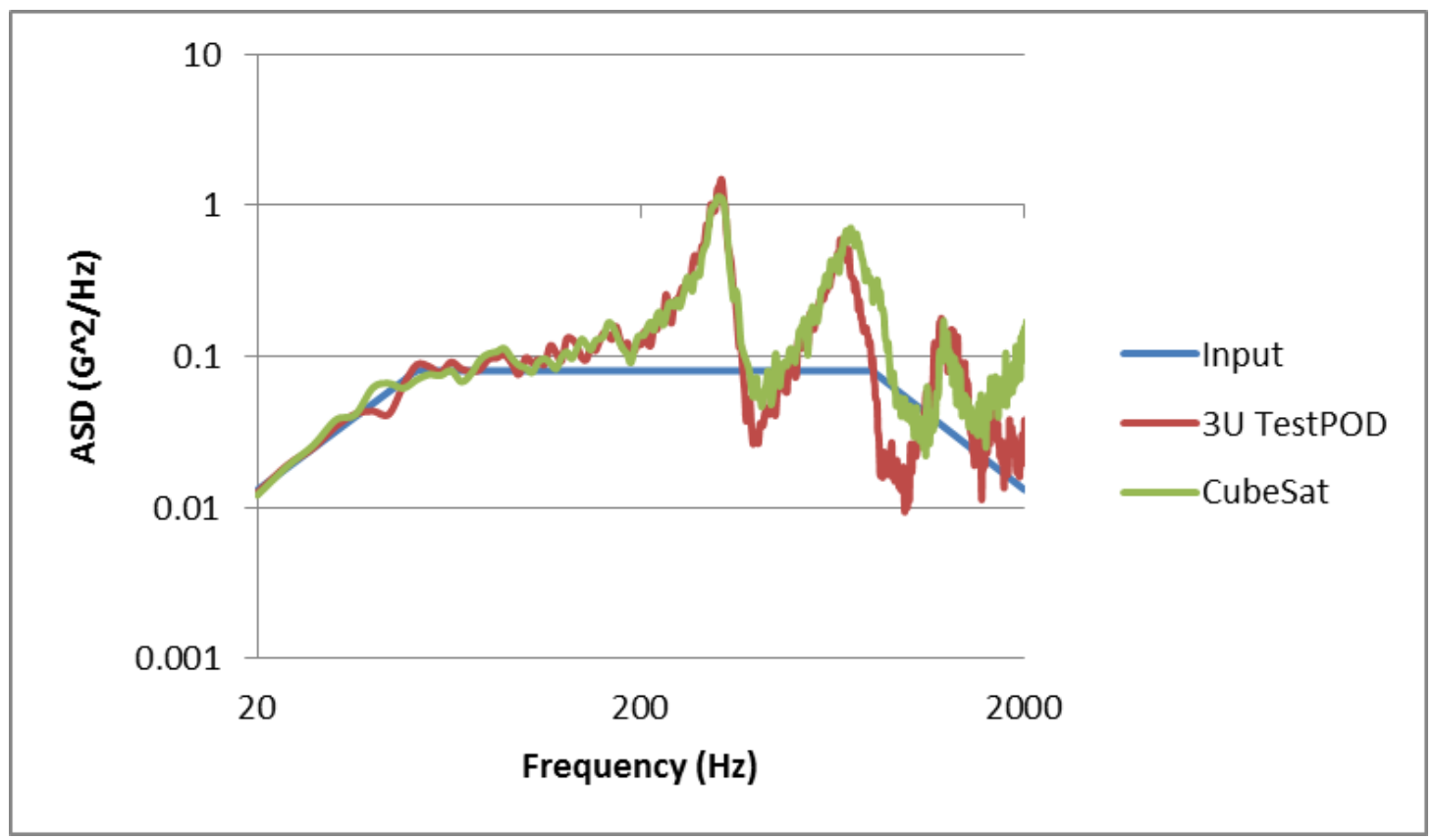

Figure 38: $\mathrm{Z}$ axis vibration data from three $1 \mathrm{~kg}$ mass models integrated into a 3U TestPOD (test numbers 11 and 12-2). 


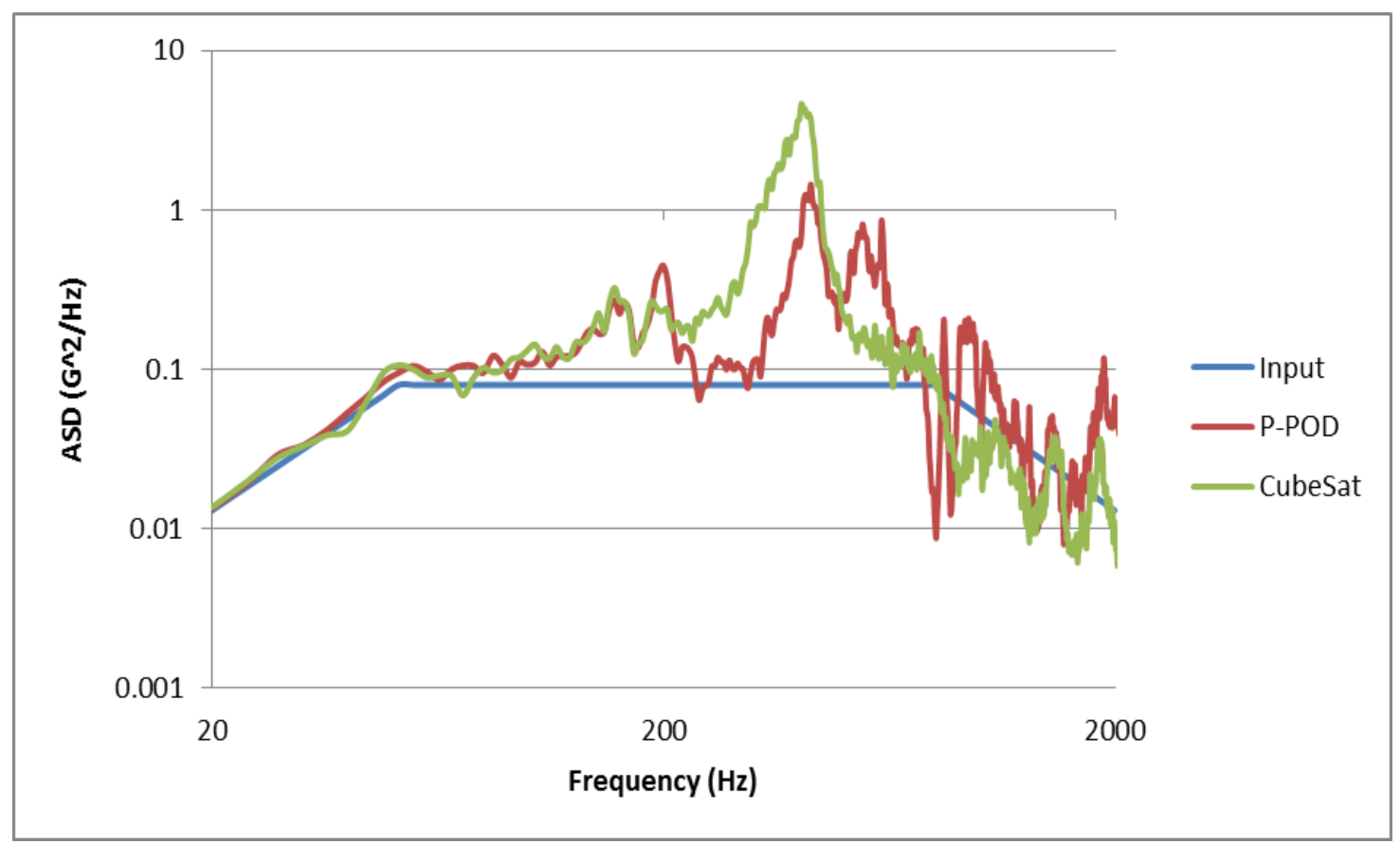

Figure 39: $\mathrm{Z}$ axis vibration data from three $1 \mathrm{~kg}$ mass models integrated into a P-POD (test numbers 17 and 18-2).

The CubeSat response follows all of the resonant peaks of the 3U TestPOD and most of the P-POD's resonant peaks, further illustrating that the CubeSats are fixed inside deployers in the $\mathrm{Z}$ axis. The P-POD has more dynamics that are not driven into the CubeSats (from plate modes or other modes with little mass participation) due to its thin outer structure. This explains why there is less coherence in P-POD and CubeSat response compared to the $1 \mathrm{U}$ and $3 \mathrm{U}$ TestPODs.

This constraint does not change with loads, which means the transmissibility in the $\mathrm{Z}$ axis is the same for any CubeSat-deployer system regardless of random vibration profile. Figure 40 shows the transmissibility of CubeSats integrated into a P-POD 
subjected to two very different random vibration profiles (GEVS Acceptance and Random Profile 1).

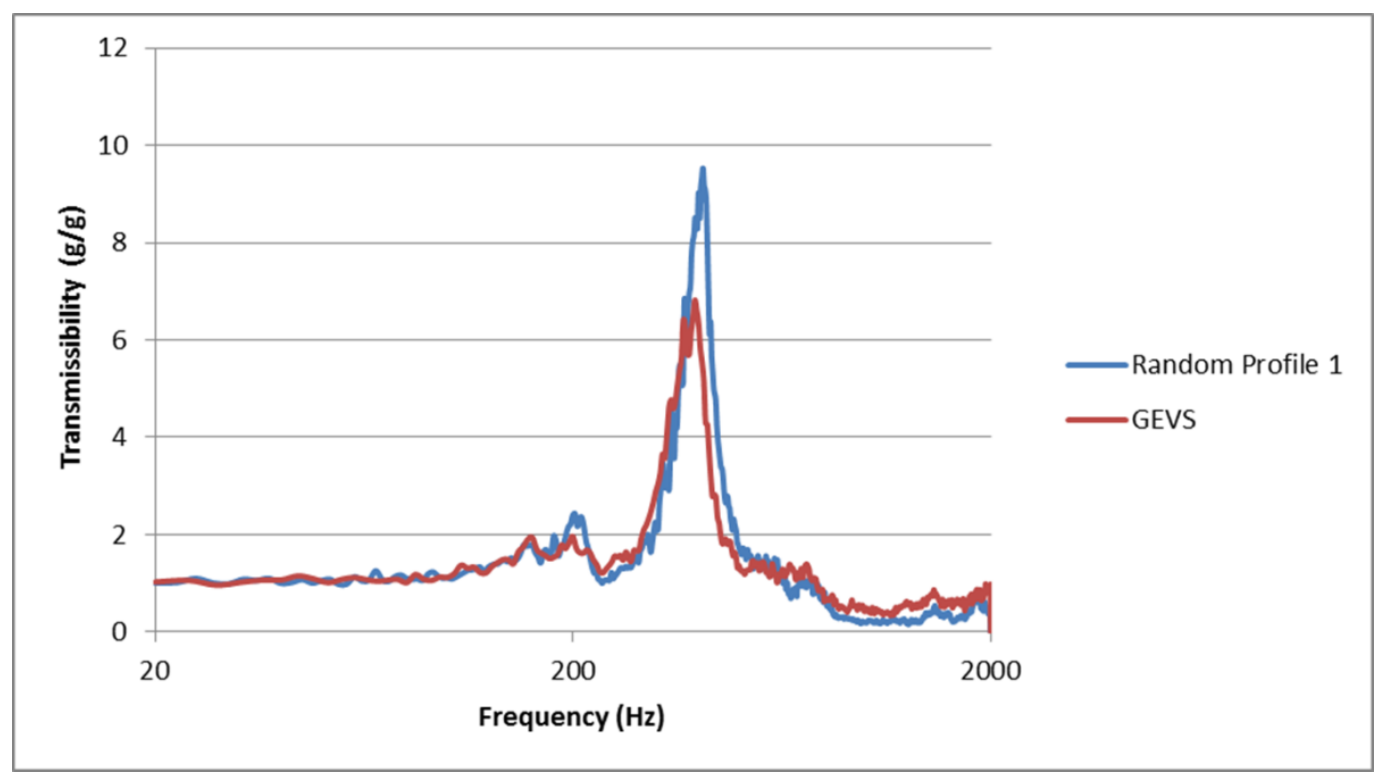

Figure 40: $\mathrm{Z}$ axis transmissibility versus frequency for CubeSats in P-POD subject to Random Vibration Profile 1 and GEVS Random Vibration Profile (test numbers 18-2 and 19).

Even though the random vibration inputs were very different, the transmissibility is very similar. This is important because it shows the constraint is independent of random vibration profile.

\section{$X$ and $Y$ Axes Constraints}

\section{Effect of Canister Constrained CubeSat}

It was shown with the $\mathrm{Z}$ axis constraints that fixed CubeSats resonate with the deployer because the deployer-CubeSat system acts as one continuous structure. However, when the CubeSat is not fixed, and is allowed to rattle around in the deployer, this is not the case. Testing was performed to understand how CubeSats respond when 
they are not fixed. Figure 41 shows the response from two $\mathrm{Z}$ axis tests in the $1 \mathrm{U}$ TestPOD: one with the CubeSat fixed by the spring plungers (standard integration), and one with the spring plungers replaced by backed out bolts. The bolts were backed out from standard integration depth by 1.5 turns to allow the CubeSats to translate in the $\mathrm{Z}$ axis. Bolts were used instead of spring plungers to mimic the solid CubeSat to deployer impact seen in the $\mathrm{X}$ and $\mathrm{Y}$ axes. This test provides a direct comparison of a fixed CubeSat with a CubeSat that can translate in the same axis of the same deployer.

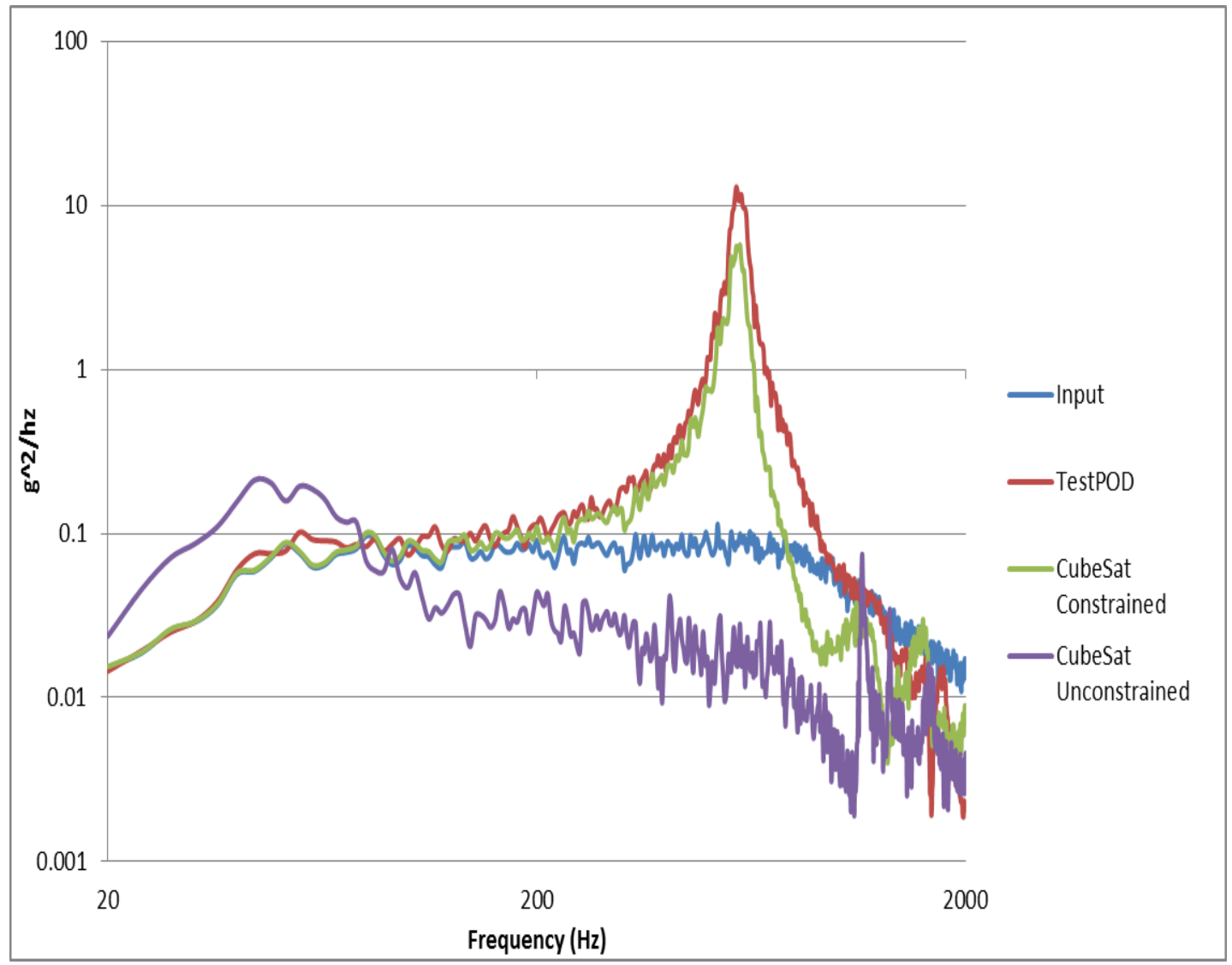

Figure 41: Fixed and unfixed CubeSat response in $Z$ axis of $1 U$ TestPOD (test numbers 3 and 4). 
The fixed CubeSat resonates with the deployer, but the unfixed CubeSat does not. When the CubeSat is allowed to displace inside the deployer, it becomes isolated from the deployer's dynamics. The unfixed response is very similar to placing an isolator between the CubeSat and deployer.

The unfixed CubeSat from Figure 41 is allowed to translate in the $\mathrm{Z}$ axis with negligible friction force. However, there is a friction force from the spring plungers that restricts CubeSat translation in the $\mathrm{X}$ and $\mathrm{Y}$ axis. This friction force can change the isolation effect of the unfixed CubeSat. The fixed and frictionless unfixed responses from Figure 41 serve as baselines for fixed and unfixed CubeSats when $\mathrm{X}$ and $\mathrm{Y}$ axes tests are run.

\section{Effect of Friction from Spring Plungers on $X$ and $Y$ Axes Constraint}

If the spring plungers are dialed in tight enough, the normal force in the $\mathrm{Z}$ axis creates a friction force in the $\mathrm{X}$ and $\mathrm{Y}$ axes so great that the CubeSat is effectively fixed inside the deployer. When the spring plungers are not dialed in as tight, the friction force is low enough that the CubeSats is unconstrained and can translate freely in the $\mathrm{X}$ and $\mathrm{Y}$ axes. Figure 42 shows how the spring plungers create restrictive friction forces in the $X$ and Y axes for CubeSats in deployers. 


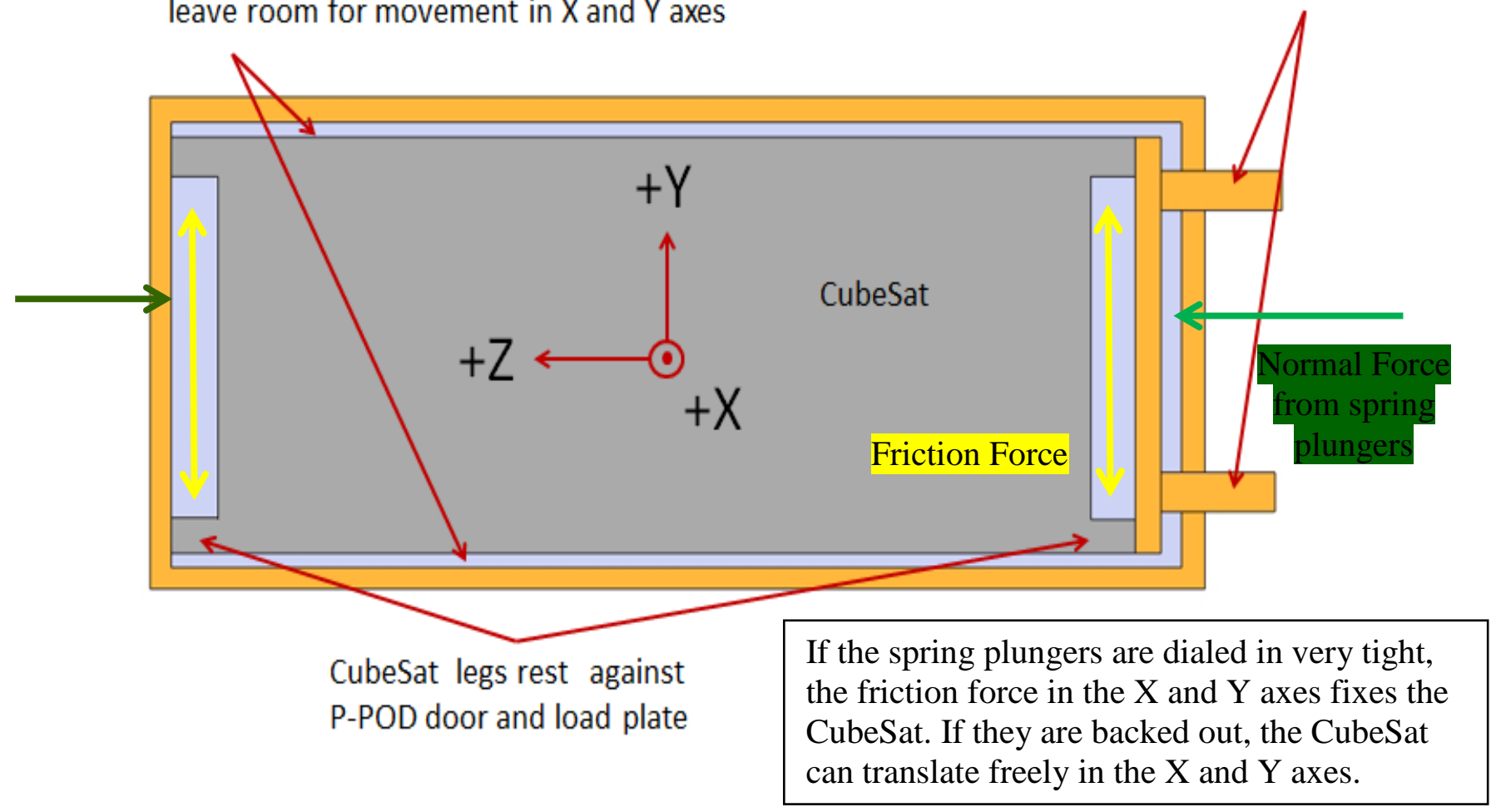

Figure 42: Schematic of how spring plungers affect the CubeSat constraint.

Several tests were run with a CubeSat mass model inside a 1U TestPOD in the $\mathrm{X}$ axis. The first test was run with the spring plungers dialed in very tight (to a torque value higher than standard CubeSat integration techniques). The second test was run with the spring plungers backed out slightly (spring plungers dialed in less than standard CubeSat integration techniques). These two tests were done to compare how friction forces in the $\mathrm{X}$ and $\mathrm{Y}$ axes affect the CubeSat constraint. The two different CubeSat resonant peaks in Figure 43 illustrate how enough friction can constrain the CubeSat and not enough friction will let the CubeSat translate inside the deployer. 


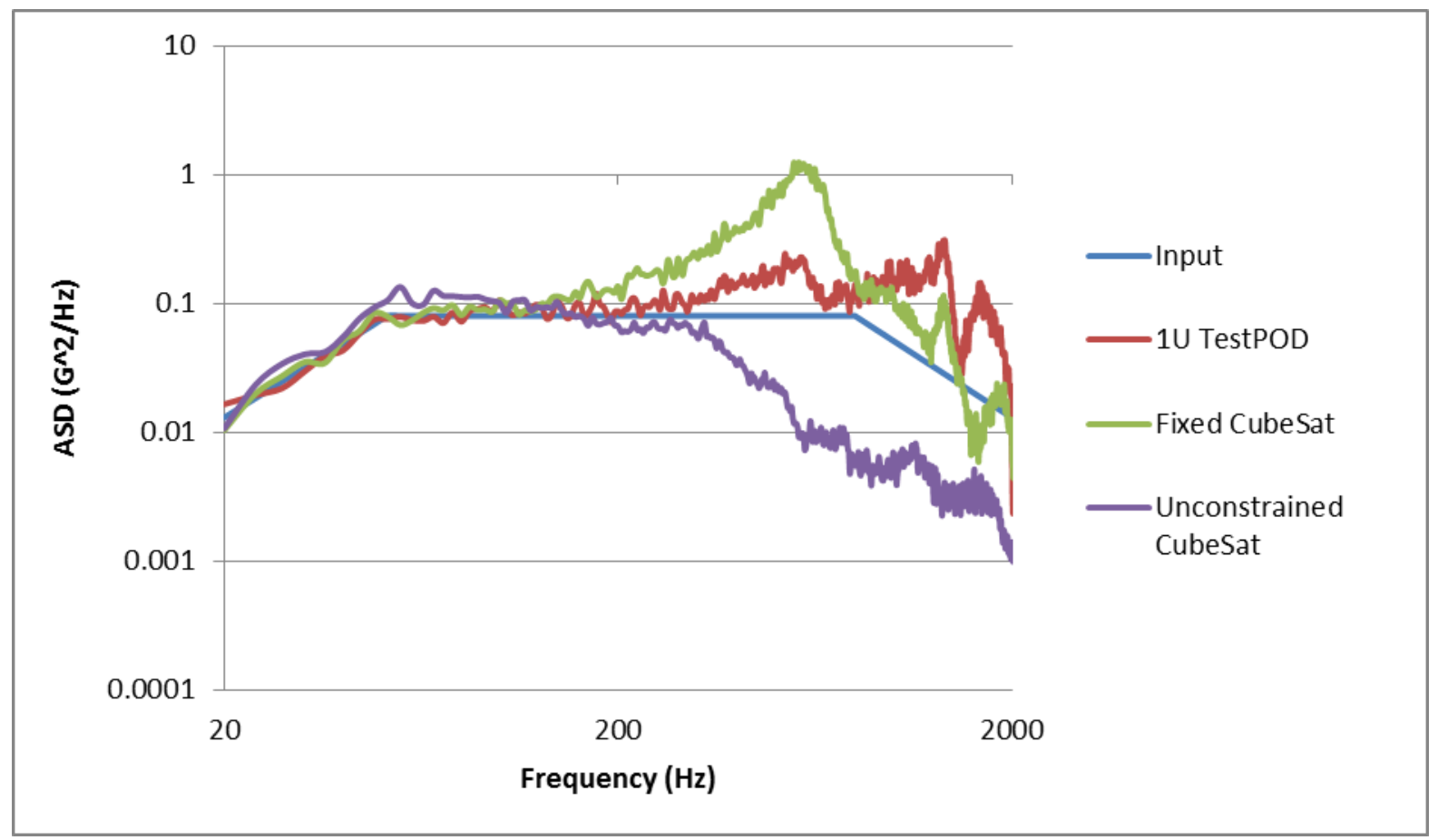

Figure 43: $\mathrm{X}$ axis vibration data for a $1 \mathrm{~kg}$ CubeSat mass model integrated into a $1 \mathrm{U}$ TestPOD with spring plungers both dialed in tight and backed out (test numbers 6, 8-1 and 9-1).

When the spring plungers are dialed in tight, the CubeSat is fixed inside the deployer, just like in the $\mathrm{Z}$ axis tests. The transfer function between the deployer and CubeSat changes significantly when the spring plungers are backed out and the CubeSat is allowed to move in the $\mathrm{X}$ axis. Because of the canister constraint, the CubeSat is allowed to translate inside the deployer, creating the isolation effect seen from the unfixed CubeSat in Figure 41. This creates a slightly greater transmissibility in the lower frequencies, but isolates the CubeSat from the deployer's dynamics in the higher frequencies.

\section{CubeSat Slip Load in $X$ and $Y$ Axes}

Vibration loads can affect whether a set friction force from spring plungers is large enough to constrain the CubeSat. Sine sweep tests at $0.55 \mathrm{~g}$ were run with the same 
exact spring plunger configurations from the tests in Figure 43. Figure 44 shows the CubeSat is fixed in the deployer for both configurations.

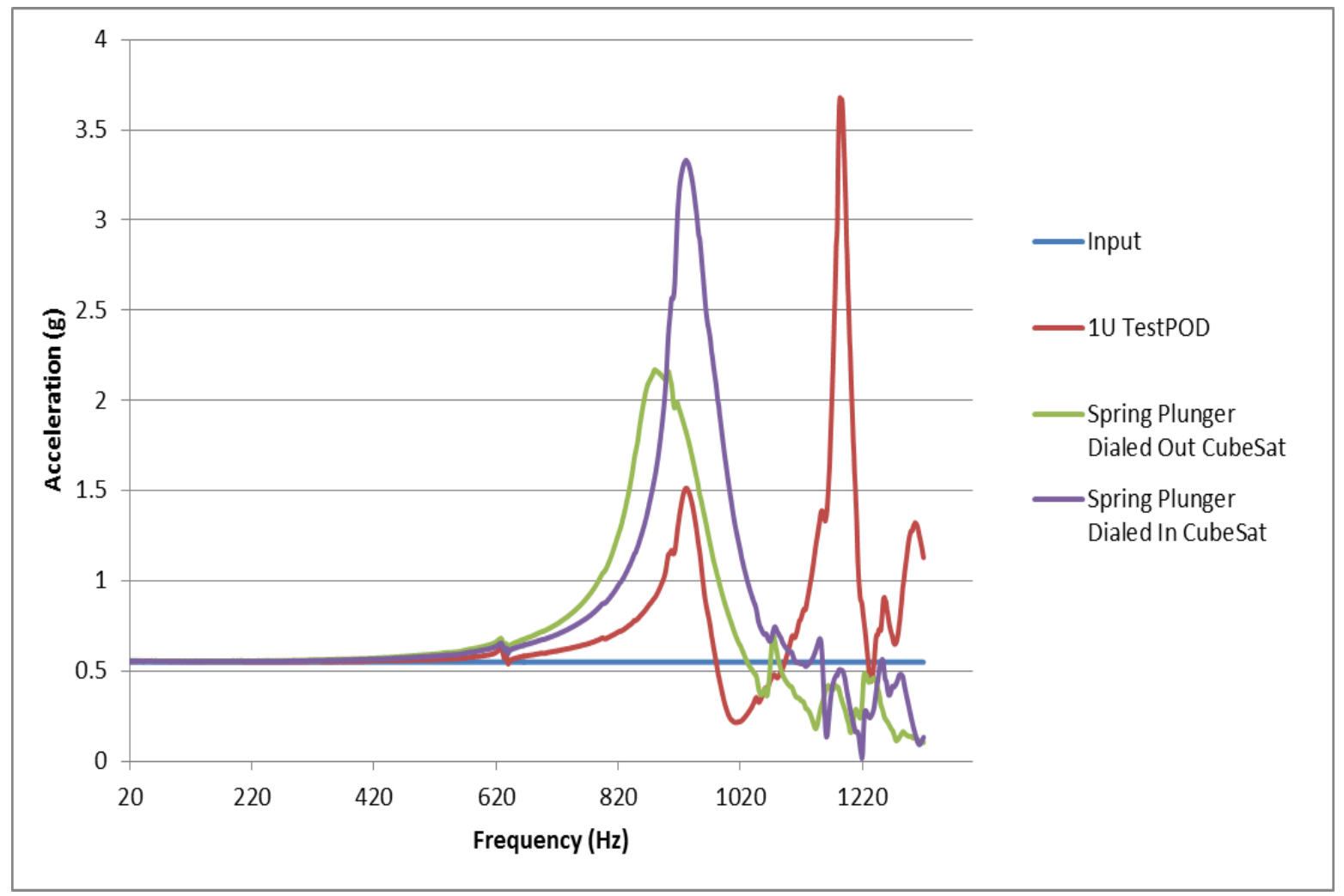

Figure 44: $\mathrm{X}$ axis sine sweep data for $1 \mathrm{~kg}$ CubeSat mass model integrated into a $1 \mathrm{U}$ TestPOD with spring plungers both dialed in tight and backed out (test numbers 5, 8-2 and 9-2).

Whether the spring plungers were dialed in or backed out, the CubeSat resonated with the deployer's first mode during the low level sine sweep, showing that the CubeSat was fixed inside the deployer and not allowed to translate. Figure 43 shows the CubeSat is isolated when the spring plungers are backed out during random vibration testing, but Figure 44 shows the CubeSat with the same spring plunger configuration is fixed inside the deployer during sine sweep testing. The friction force from the backed out spring plungers was the same for both tests, but the random vibration test from Figure 43 was at 
about $10 \mathrm{G}_{\mathrm{rms}}$ and the sine test from Figure 44 was at $0.55 \mathrm{~g}$. The friction force from the backed out spring plungers was large enough to fix the CubeSat during the sine sweep, but not large enough to fix it during the random vibration test.

When the vibration load is high enough that the friction from the spring plungers can no longer constrain the CubeSat in the $\mathrm{X}$ and $\mathrm{Y}$ axes, the CubeSat has reached its "slip load." If the vibration load is lower than the slip load, the CubeSat is fixed inside the deployer. If loads are higher than the slip load, the CubeSat can translate in the $\mathrm{X}$ and $\mathrm{Y}$ axes and the isolation effect is seen. The slip load can be raised by dialing in the spring plungers more, or lowered by backing out the spring plungers. High coefficients of friction on the CubeSat and deployer's Z axis would also raise the slip load. CubeSats with more mass lower the slip load due to the added inertia of the CubeSat.

Consider again the tests from Figure 43 and Figure 44. When the spring plungers are backed out, the slip load is somewhere between $0.55 \mathrm{~g}$ and ten $\mathrm{G}_{\mathrm{rms}}$. This is because the CubeSat is fixed at $0.55 \mathrm{~g}$ but not fixed at about ten $\mathrm{G}_{\mathrm{rms}}$. When the spring plungers are dialed in tight, the slip load is higher than ten $\mathrm{G}_{\mathrm{rms}}$ because the CubeSat is still fixed at this load. Note that this thesis did not make any attempts to accurately characterize levels for the slip load.

\section{$X$ and $Y$ Axes Constraint with Standard Integration}

Tests were run with CubeSats integrated into the three deployers using the standard CubeSat integration technique (dialing in spring plungers until they are completely bottomed out, but no further). These tests characterize the $\mathrm{X}$ and $\mathrm{Y}$ axes constraints under nominal conditions. 
Figure 45 shows CubeSat response data for a $1 \mathrm{~kg}$ CubeSat mass model integrated into a $1 \mathrm{U}$ TestPOD. This figure shows how the response varies over time.

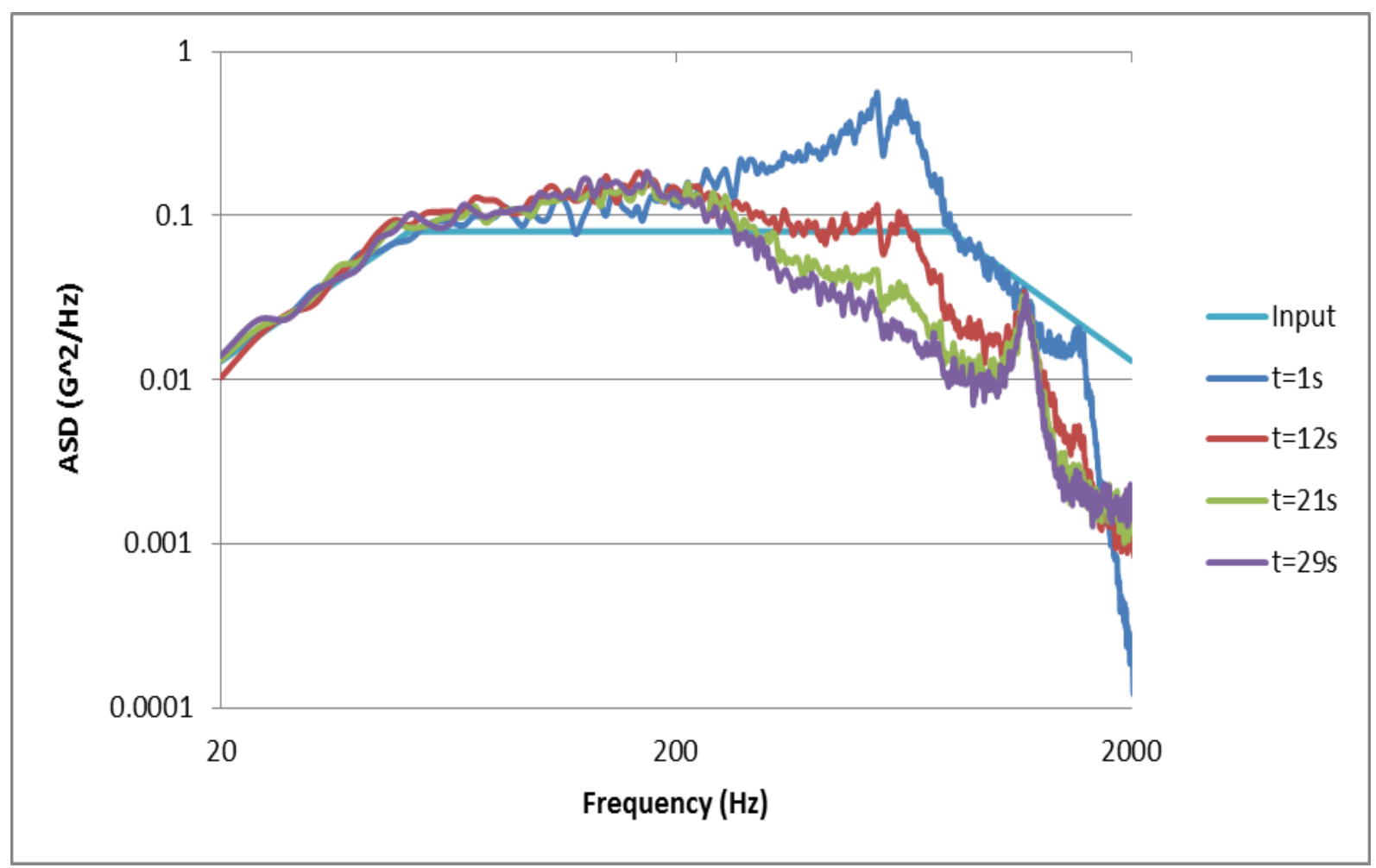

Figure 45: $\mathrm{X}$ axis vibration data for a $1 \mathrm{~kg}$ CubeSat mass model integrated into a $1 \mathrm{U}$ TestPOD with standard spring plunger integration (test number 7).

At the beginning of the tests, the typical response data is seen for a CubeSat fixed inside a 1U TestPOD. However, as the test progresses, the response shifts to display the typical response of a CubeSat that is isolated. Figure 46 shows the beginning and end CubeSat response as well as the time-independent $1 \mathrm{U}$ TestPOD response. The data from Figure 46 can be compared to the fixed and unconstrained data from Figure 43. 


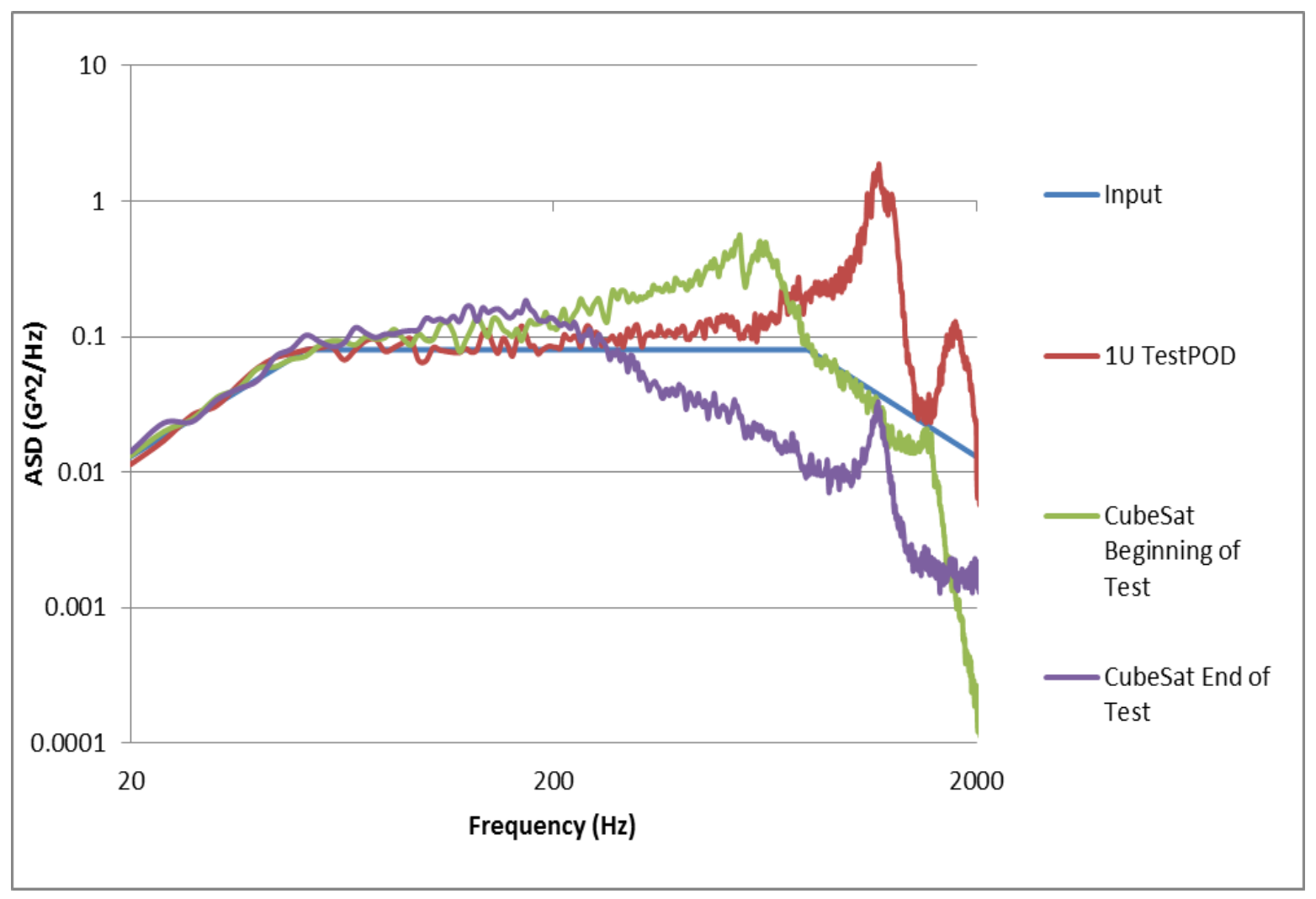

Figure 46: $\mathrm{X}$ axis vibration data for a $1 \mathrm{~kg}$ CubeSat mass model integrated into a $1 \mathrm{U}$ TestPOD at beginning and end of test (test numbers 6 and 7).

This data shows that the slip load changes over time. The input load from Figure 45 is constant, but the slip load becomes lower than the input as the test progresses. This means that the CubeSat starts above the slip load, and is fixed inside the deployer at the beginning of the test. After the tests runs for about 20 to 30 seconds, the slip load lowers enough that the CubeSat is no longer fixed, and the CubeSat settles into an isolated position in the deployer. After the CubeSat settles, the data remains consistent no matter how long the test is run. However, when the same profile from the test shown in Figure 45 was rerun, the same result was seen: the CubeSat started fixed again in the deployer and then became isolated after about 20-30 seconds. 
The same result is seen for CubeSats in the 3U TestPOD. Figure 47 shows $X$ axis vibration data for three $1 \mathrm{~kg}$ CubeSat mass models integrated into a 3U TestPOD.

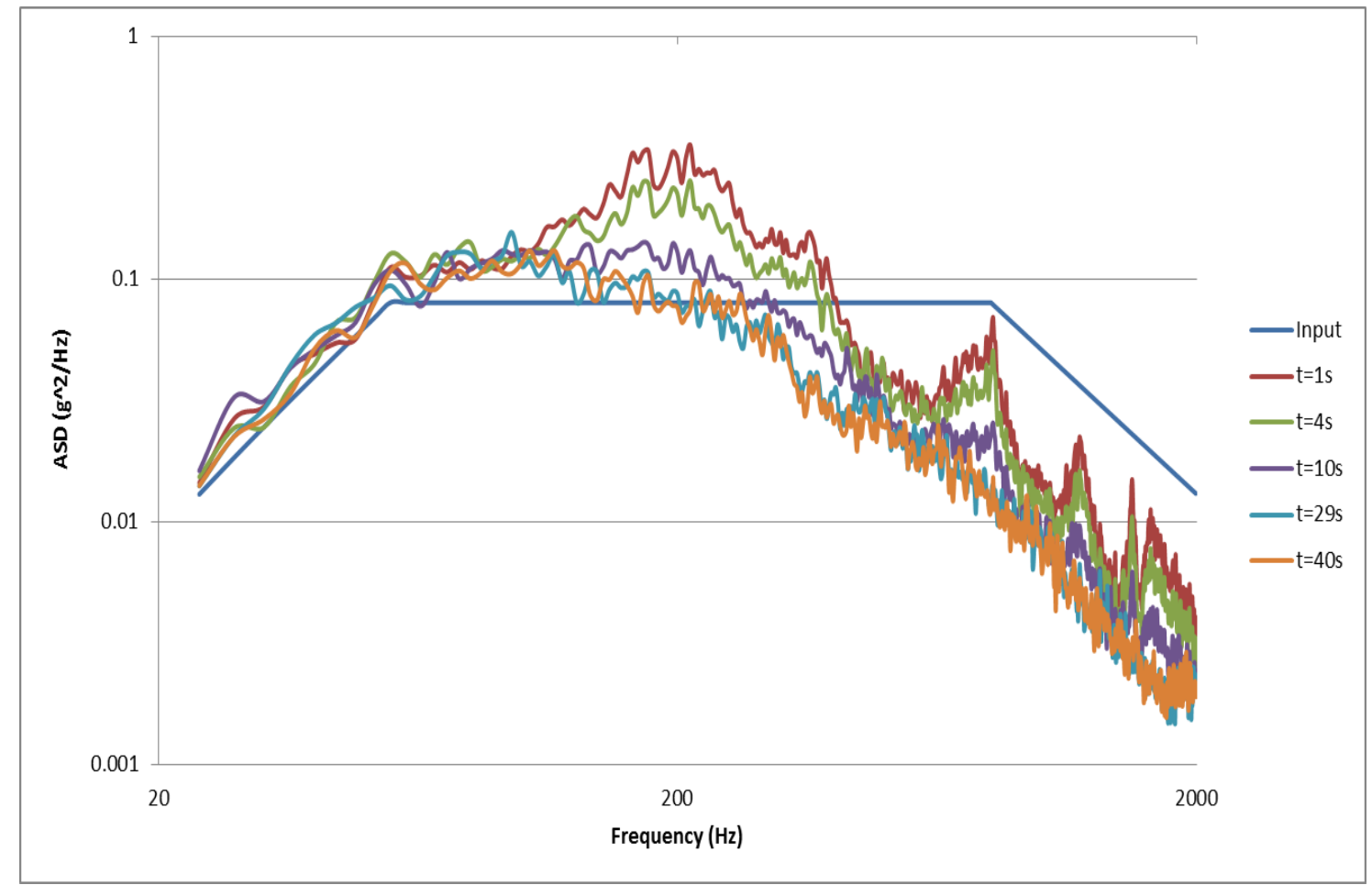

Figure 47: $\mathrm{X}$ axis vibration data for three $1 \mathrm{~kg}$ CubeSat mass models integrated into a $3 \mathrm{U}$ TestPOD with standard spring plunger integration (test number 15-2).

The CubeSat mass model response follows the 3U TestPOD's dynamics at the beginning of the test, a characteristic of the CubeSats being fixed inside the deployer. As the test continues, the CubeSat response appears to be isolated from the deployer's dynamics. Just like with the CubeSat mass model in the 1U TestPOD, the CubeSat mass models in the $3 \mathrm{U}$ TestPOD start fixed and then settle into an isolated position inside the deployer. 
Figure 48 shows the beginning and end response data of three CubeSat mass models in the 3U TestPOD as well as the 3U TestPOD's response.

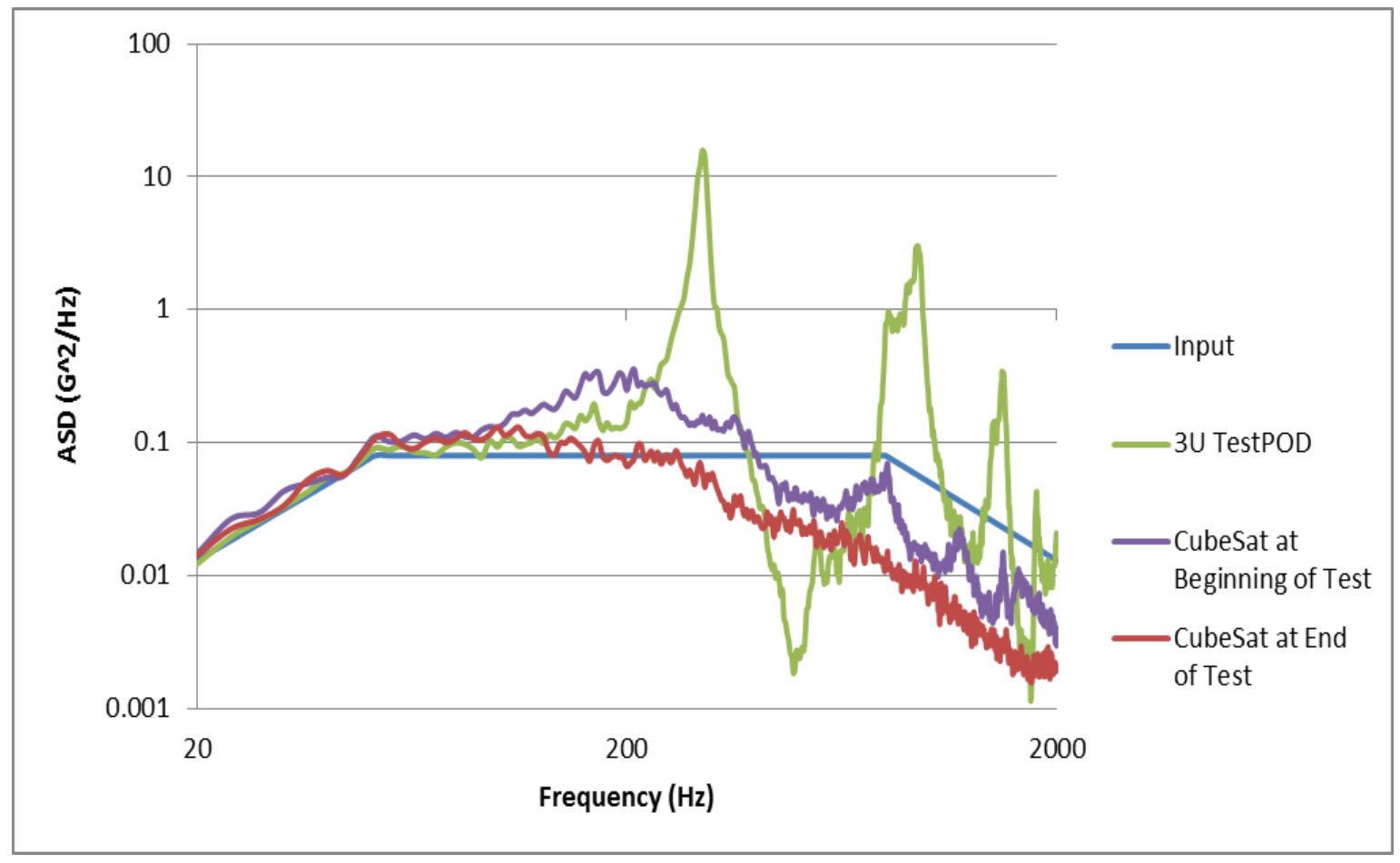

Figure 48: $\mathrm{X}$ axis vibration data for three $1 \mathrm{~kg}$ CubeSat mass models integrated into a $3 \mathrm{U}$ TestPOD at beginning and end of test (test numbers 14 and 15-2).

Compared to the CubeSat response in the 1U TestPOD shown in Figure 46, the CubeSat from Figure 48 did not respond much with the deployer at the beginning of the test. Before the loads were increased to the profile's full levels (during vibration table rampup), the fixed CubeSat had already started to become isolated. It makes sense that fixedto-isolated transition would occur at lower levels in the 3U TestPOD than the $1 \mathrm{U}$ TestPOD because there was more CubeSat mass in the 3U TestPOD. This added mass lowers the slip load, making the fixed-to-isolated transition occur during the ramp up for the $3 \mathrm{U}$ TestPOD instead of at full levels as with the $1 \mathrm{U}$ TestPOD. 
The same tests were run with CubeSat mass models in the $\mathrm{X}$ axis of the P-POD, and the same results were seen. Figure 49 shows CubeSat response change over time in the $\mathrm{X}$ axis of the P-POD. Figure 50 shows the beginning and end response data from Figure 49 along with the P-POD's time-independent response.

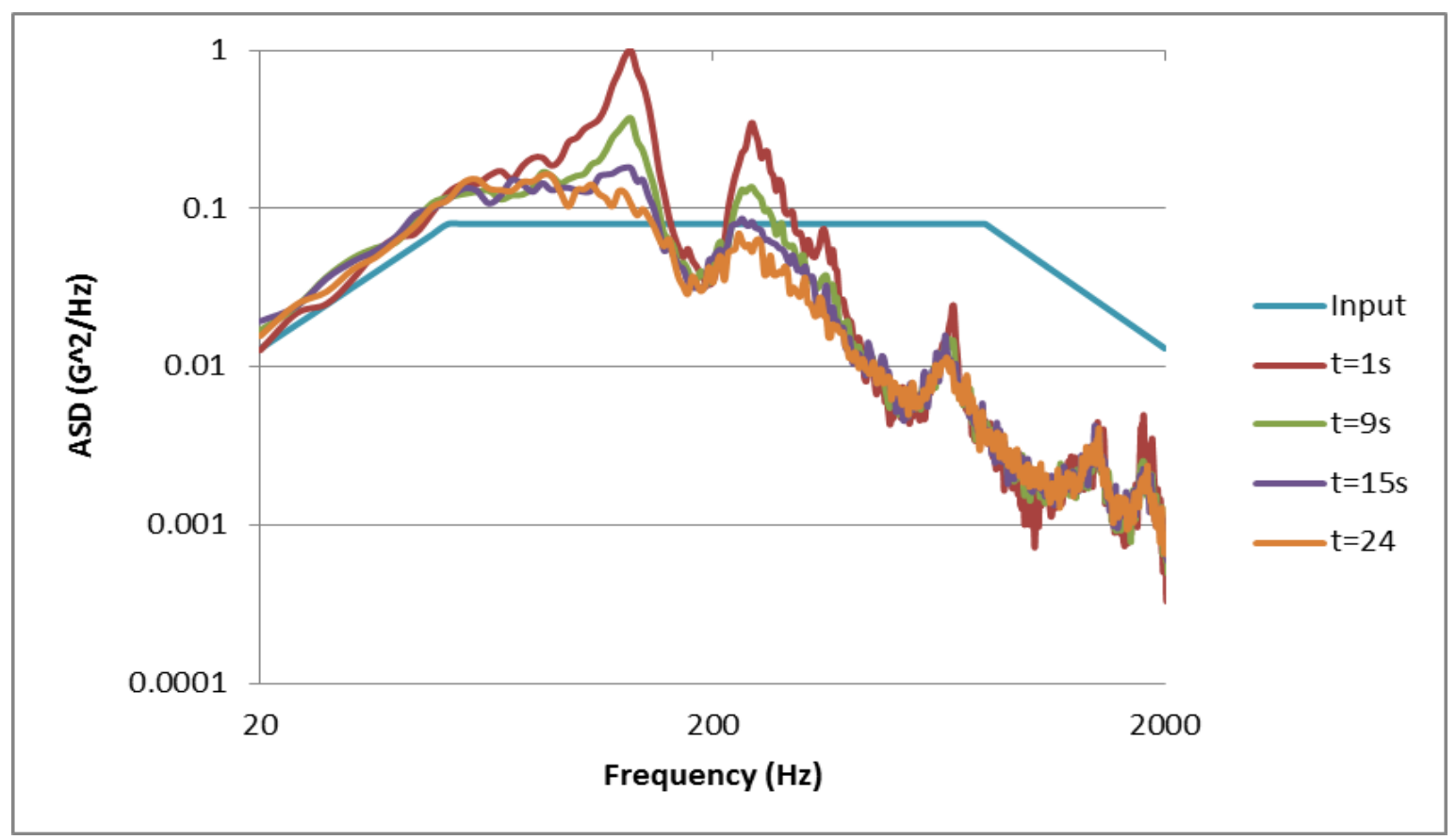

Figure 49: $\mathrm{X}$ axis vibration data for three $1 \mathrm{~kg}$ CubeSat mass models integrated into a P-POD with standard spring plunger integration (test number 22-2). 


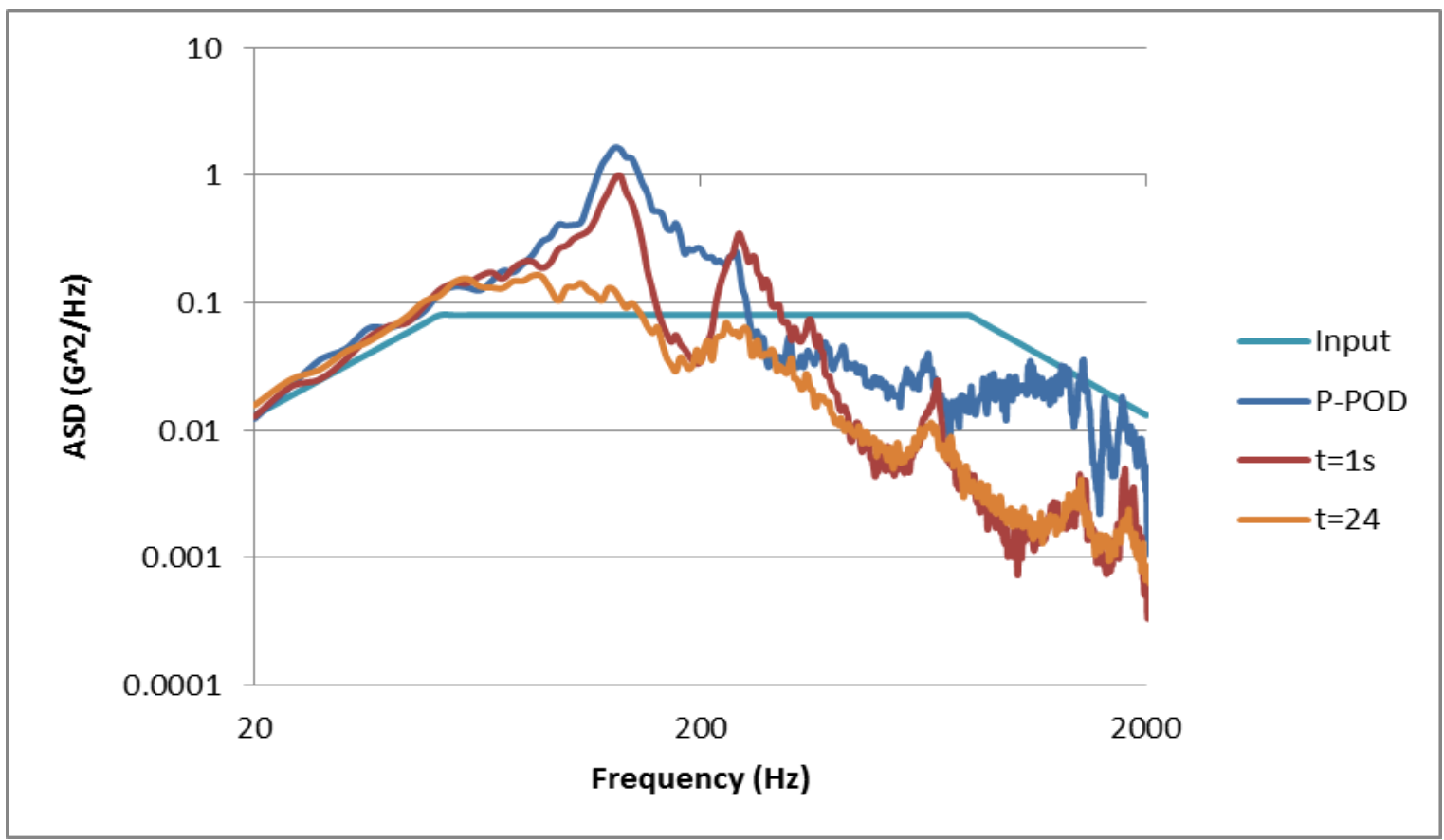

Figure 50: $\mathrm{X}$ axis vibration data for three $1 \mathrm{~kg}$ CubeSat mass models integrated into a P-POD at beginning and end of test (test numbers 21 and 22-2).

\section{Constraint Conclusion}

The experimental data shows that:

- CubeSats can be modeled as fixed inside the deployer in the $\mathrm{Z}$ axis

- Constraint is independent of random vibration profile, deployer design, or CubeSat design

- In the $\mathrm{X}$ and $\mathrm{Y}$ axes:

- CubeSats are fixed if slip load is higher than vibration load

- CubeSats are isolated if vibration load is higher than slip load

- The slip load can change during vibration testing 
- CubeSats generally start fixed, then settle into an isolated position inside the deployer

By dialing in the spring plungers, CubeSats are effectively fixed in the $\mathrm{Z}$ axis with no significant transfer function between the deployer and the CubeSats. The normal force created by the spring plungers creates a friction force in the $\mathrm{X}$ and $\mathrm{Y}$ axes. This

force usually fixes the CubeSat at the beginning of the test. As the test progresses, the CubeSat overcomes the friction force and is allowed to translate in the $\mathrm{X}$ and $\mathrm{Y}$ axes. When the CubeSat is fixed at the beginning of the test, there is no significant transfer function between the deployer and CubeSat. When the CubeSat is allowed to translate, it becomes isolated from the deployer, creating a significant transfer function between the deployer and CubeSat.

\section{Developing a Vibration Model for the CubeSat-Deployer System}

\section{Z Axis Model}

\section{Load Path Model}

CubeSats are constrained in the $\mathrm{Z}$ axis, and can be modeled as fixed inside the deployer. This means the CubeSat-deployer system can be modeled as one continuous structure. Because there is only one structure, only one FRF is needed to obtain the steady state frequency response of the CubeSat. Figure 51 shows a block diagram of the load path from the random vibration profile to the CubeSat response. 


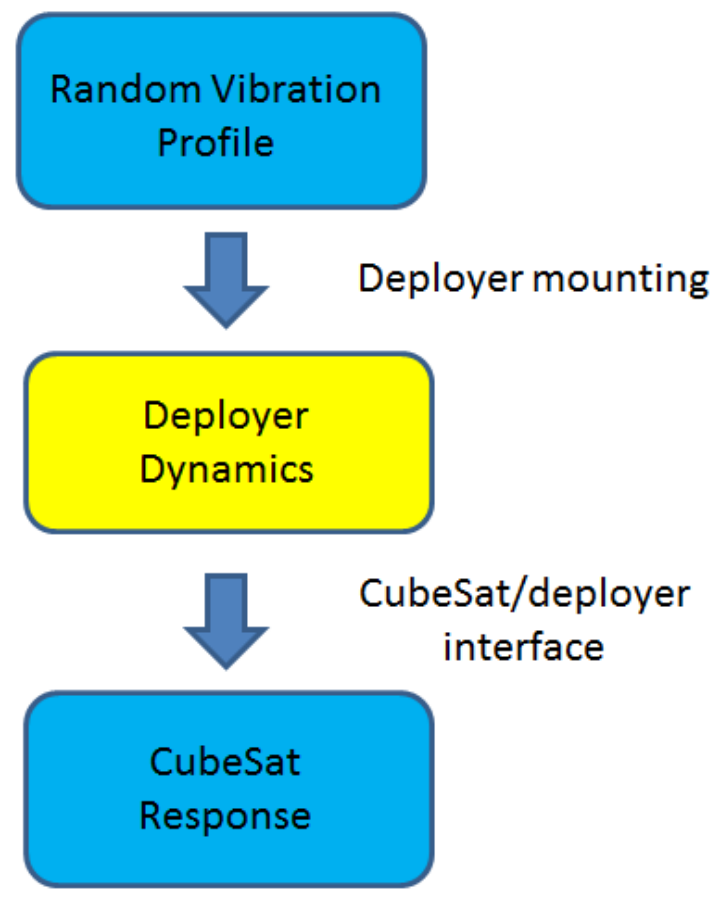

Figure 51: $\mathrm{Z}$ axis load path from vibration input to CubeSat load.

The yellow block represents the only transfer function between the vibration input and the CubeSat response. The deployer mounting and CubeSat/deployer interface are considered one to one. Note that the CubeSat mass, stiffness and damping can affect the deployer's dynamics.

\section{Transfer Function (FRF) Model}

To create a model for CubeSat loads, the transfer function (FRF) from the load path in Figure 51 is needed. Linear vibration theory provides a sufficiently accurate model for this transfer function, as the transmissibility is independent of load magnitude (see Figure 40). The FRF can be approximated using experimental data from a characterized deployer, a finite element model of a deployer, or, if the deployer is 
assumed to only have one peak in the $\mathrm{Z}$ axis, using SDOF linear vibration theory (or Miles' equation).

To approximate the FRF using SDOF linear vibration theory, the system's natural frequency and damping ratio are needed. These parameters are used with Equation 6 to obtain the steady state FRF. The natural frequency can be obtained through vibration testing. Because the CubeSat mass and stiffness can affect the system's natural frequency, a range of CubeSats would have to be tested to obtain a full characterization of the CubeSat-deployer system natural frequencies. Finite Element Analysis (FEA) could also be used to model the deployer and CubeSat and find the system's natural frequency using a "normal modes" solution. The damping ratio could be approximated or obtained experimentally through characterization. A range of CubeSats with different amounts of damping would need to be characterized to obtain an accurate estimate of the damping ratio for the FRF. If only the equivalent static load is desired and not the frequency response, the natural frequency and damping information can be used with Miles' equation.

Consider the $1 \mathrm{~kg}$ CubeSat mass model integrated into the $1 \mathrm{U}$ TestPOD. If the $1 \mathrm{U}$ TestPOD is assumed to only have one peak, SDOF linear vibration theory can be used to model the CubeSat response. Figure 52 shows that if a good estimate of the natural frequency and damping ratio are used, an accurate FRF can be obtained until the first peak. 


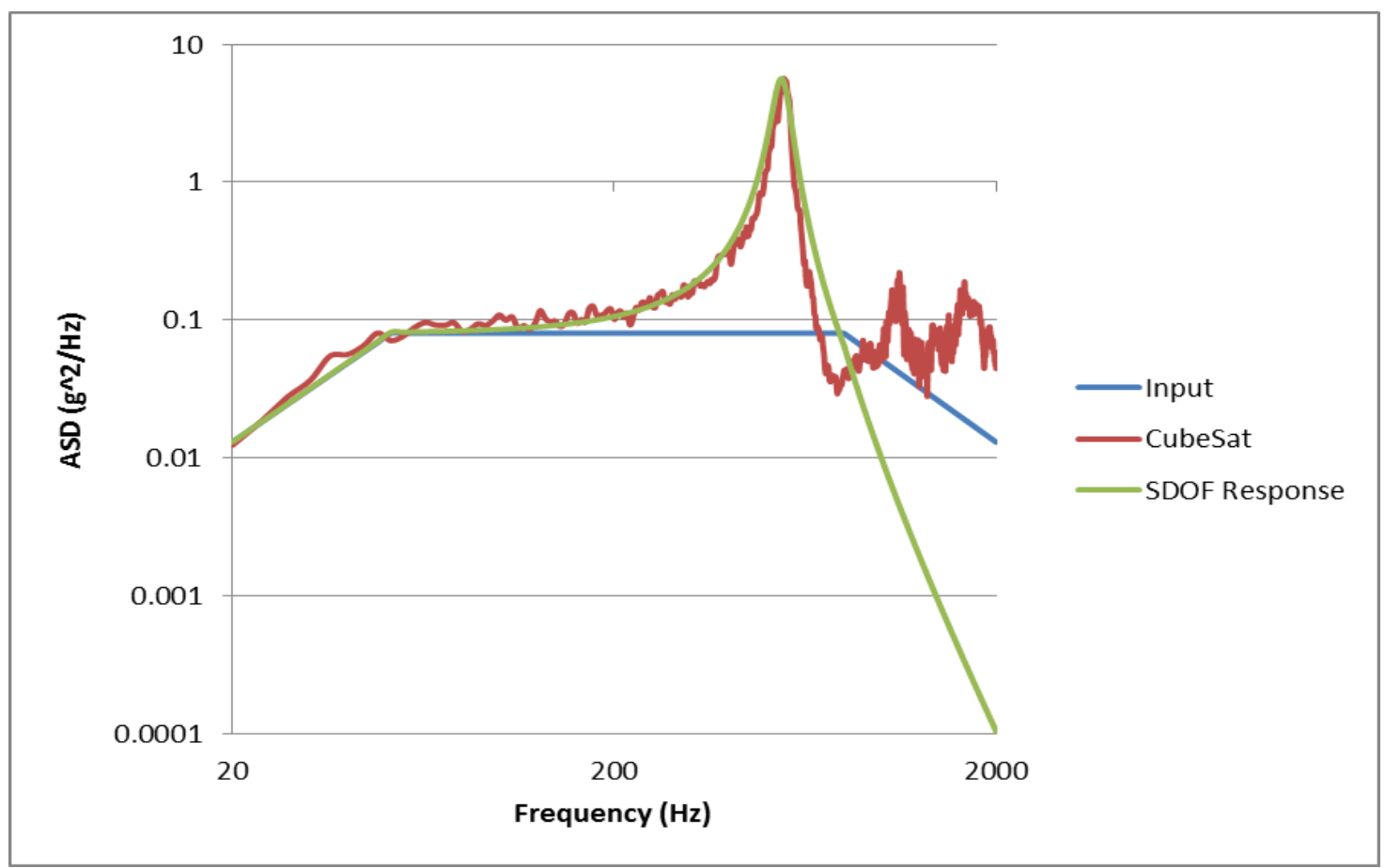

Figure 52: CubeSat response compared to SDOF model $\left(\omega_{n}=540 \zeta=.06\right)$ in a 1U TestPOD (test number 3).

Because there are actually multiple resonant peaks for the deployer, the SDOF model is not accurate after the first peak. This is because the SDOF model enters into the region of isolation while the true CubeSat response has further amplification due to additional resonant peaks.

Figure 53 and Figure 54 show the SDOF model used to approximate CubeSat response inside the $3 \mathrm{U}$ TestPOD and P-POD. Like with the $1 \mathrm{U}$ TestPOD, the model is a good approximation until after the first peak. The SDOF model assumes a region of isolation after the first peak when the 3U TestPOD and P-POD actually have multiple peaks. 


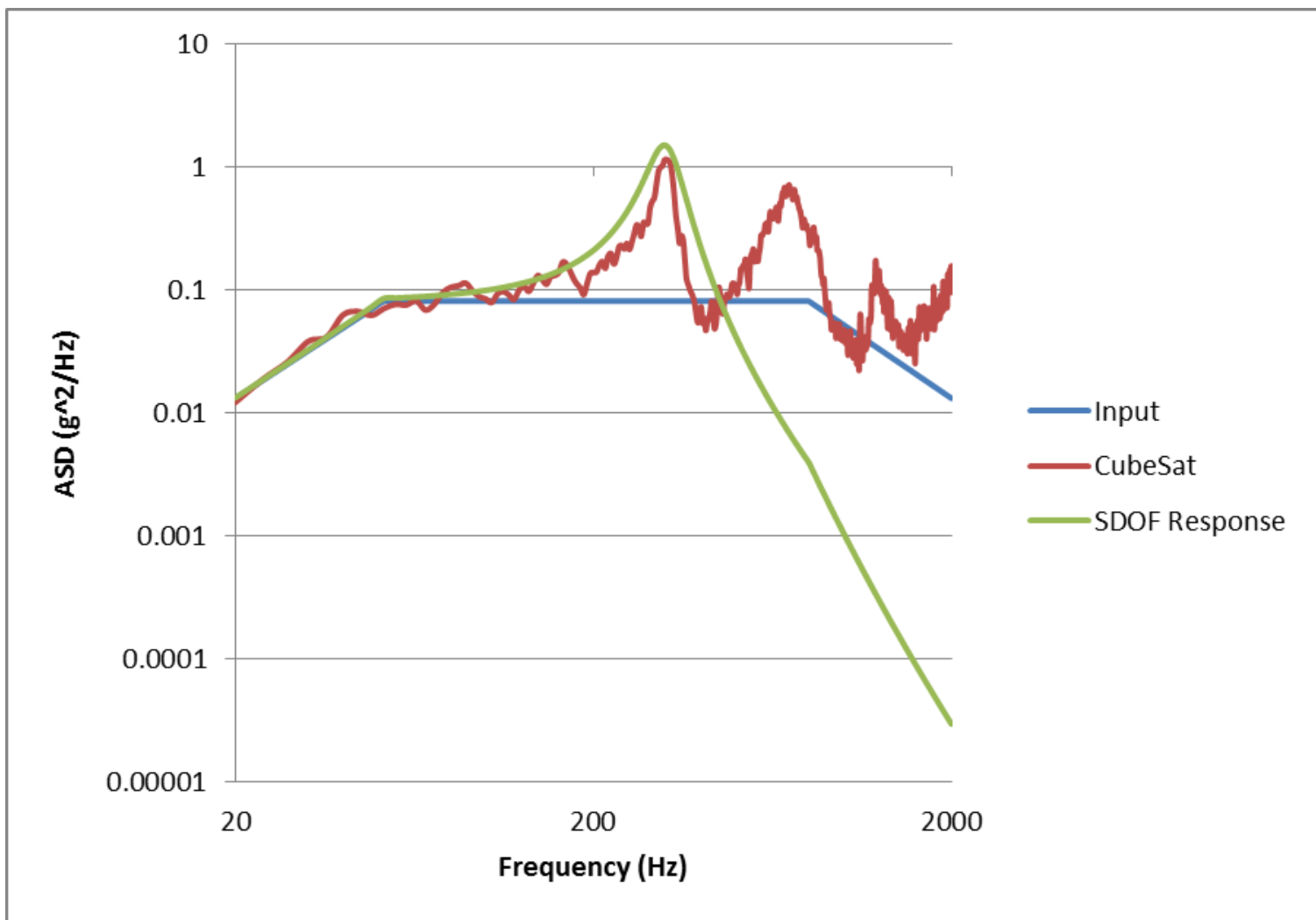

Figure 53: CubeSat response compared to SDOF model $\left(\omega_{n}=320 \zeta=.12\right)$ in a 3U TestPOD (test number 12-2). 


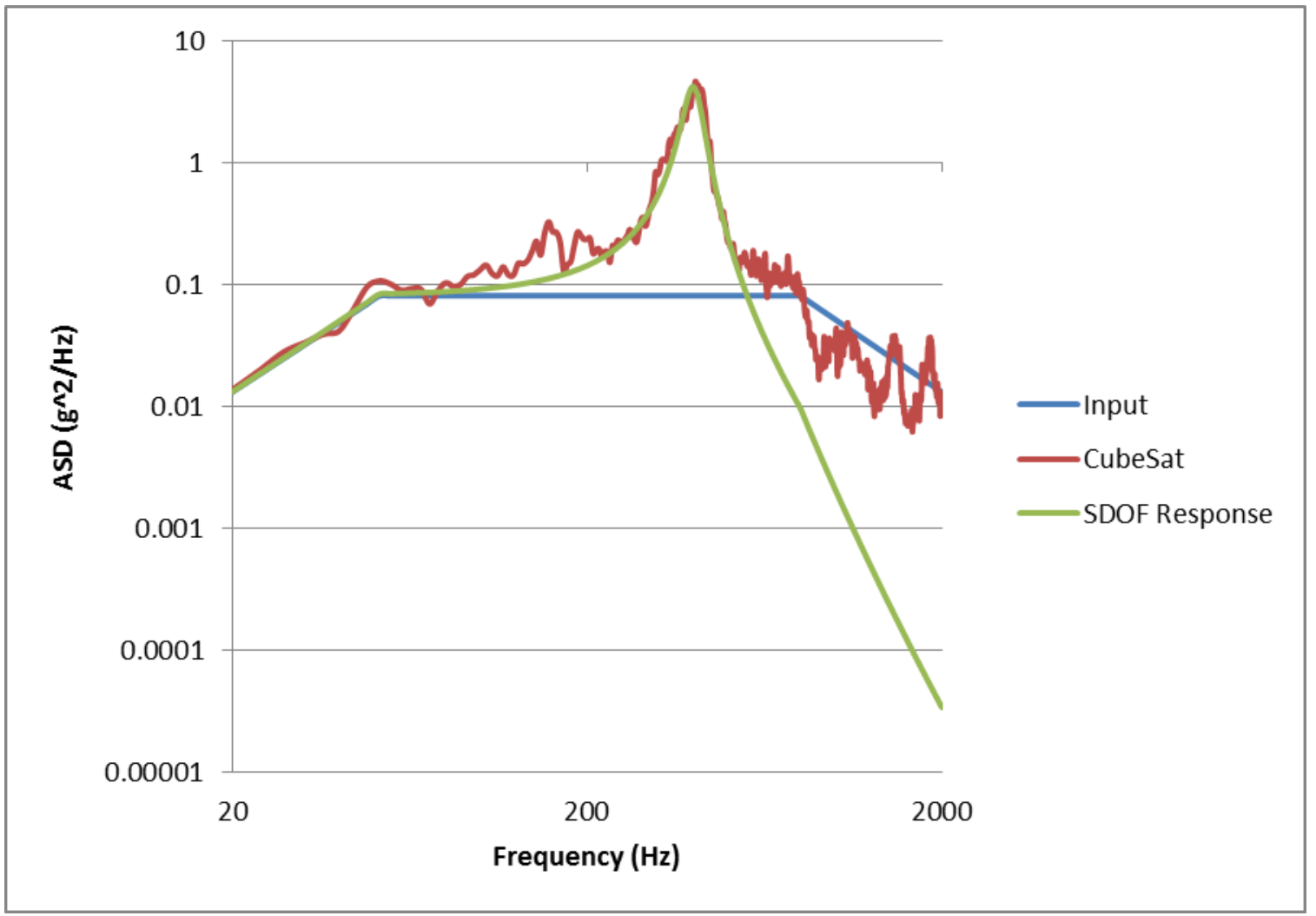

Figure 54: CubeSat response compared to SDOF model $\left(\omega_{n}=400 \zeta=0.1\right)$ in a P-POD (test number 18-2).

The SDOF model examples illustrates that, with accurate damping and natural frequency information, the frequency response can be obtained until the first peak. The SDOF model fitting the experimental data also provides analytical verification that there is only one transfer function between the vibration input and the CubeSat, and that there is no transfer function due to the $\mathrm{Z}$ axis constraint. This provides an important analytical validation of the $\mathrm{Z}$ axis vibration model.

While the SDOF estimate for the FRF is the simplest method, it is only a good model until the first peak. Because there are most likely multiple peaks for a deployer, 
the SDOF model will underestimate loads past the first peak. This FRF model, however, is good for quickly approximating the peak response at resonant frequency.

For more accurate results, a MDOF FRF is needed. This can be obtained through experimental data or FEA. The transfer function can be obtained through sine sweep tests or other vibration tests. Because the system is assumed to be linear, the transmissibility from past tests of CubeSats integrated in the deployer will work as the FRF (assuming the CubeSat mass, stiffness and damping are relatively close).

Characterization of a deployer with CubeSats of different mass, stiffness and damping can provide accurate transfer function for all types of CubeSats. The frequency response can also be obtained from FEA using a "random response" solution, but the damping ratio must still be approximated or obtained experimentally.

$X$ and $Y$ Axes Model

\section{Load Path Model}

CubeSats can be fixed or isolated in the $\mathrm{X}$ and $\mathrm{Y}$ axes. When the CubeSat is isolated, the CubeSat-deployer system cannot be modeled as one continuous structure, and an additional transfer function is added to the load path from vibration input to the CubeSat. Figure 55 shows a block diagram of the load path in the $\mathrm{X}$ and $\mathrm{Y}$ axes. 


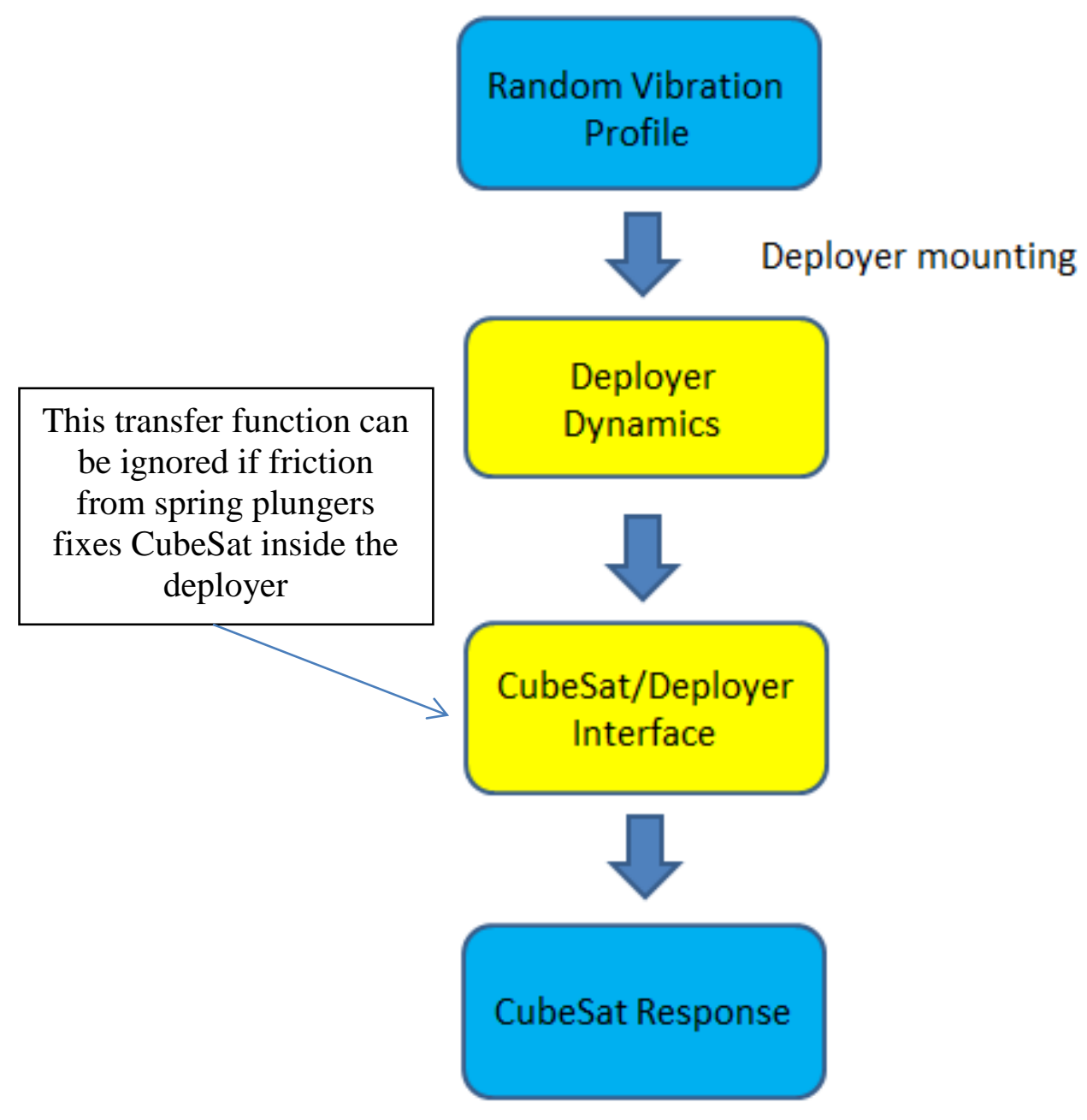

Figure 55: Block diagram for $\mathrm{X}$ and $\mathrm{Y}$ axes load path.

The yellow blocks represent transfer functions, or FRFs, between the vibration input and CubeSat response. It can generally be assumed that these two transfer functions do not couple with one another. If the vibration inputs are lower that the slip loads and the CubeSat is fixed inside the deployer (as with low level sine sweep tests), the 
CubeSat/Deployer interface block can be taken out of the flow chart, as the transfer function would be one to one.

\section{Transfer Function (FRF) Model}

If the CubeSat is fixed inside the deployer from spring plunger friction, there is no deployer-CubeSat interface transfer function. The CubeSat response can be modeled with just one transfer function that is based on deployer dynamics, like the $\mathrm{Z}$ axis. Figure 56 shows the SDOF approximation of $\mathrm{X}$ axis response for a CubeSat fixed inside a $1 \mathrm{U}$ TestPOD.

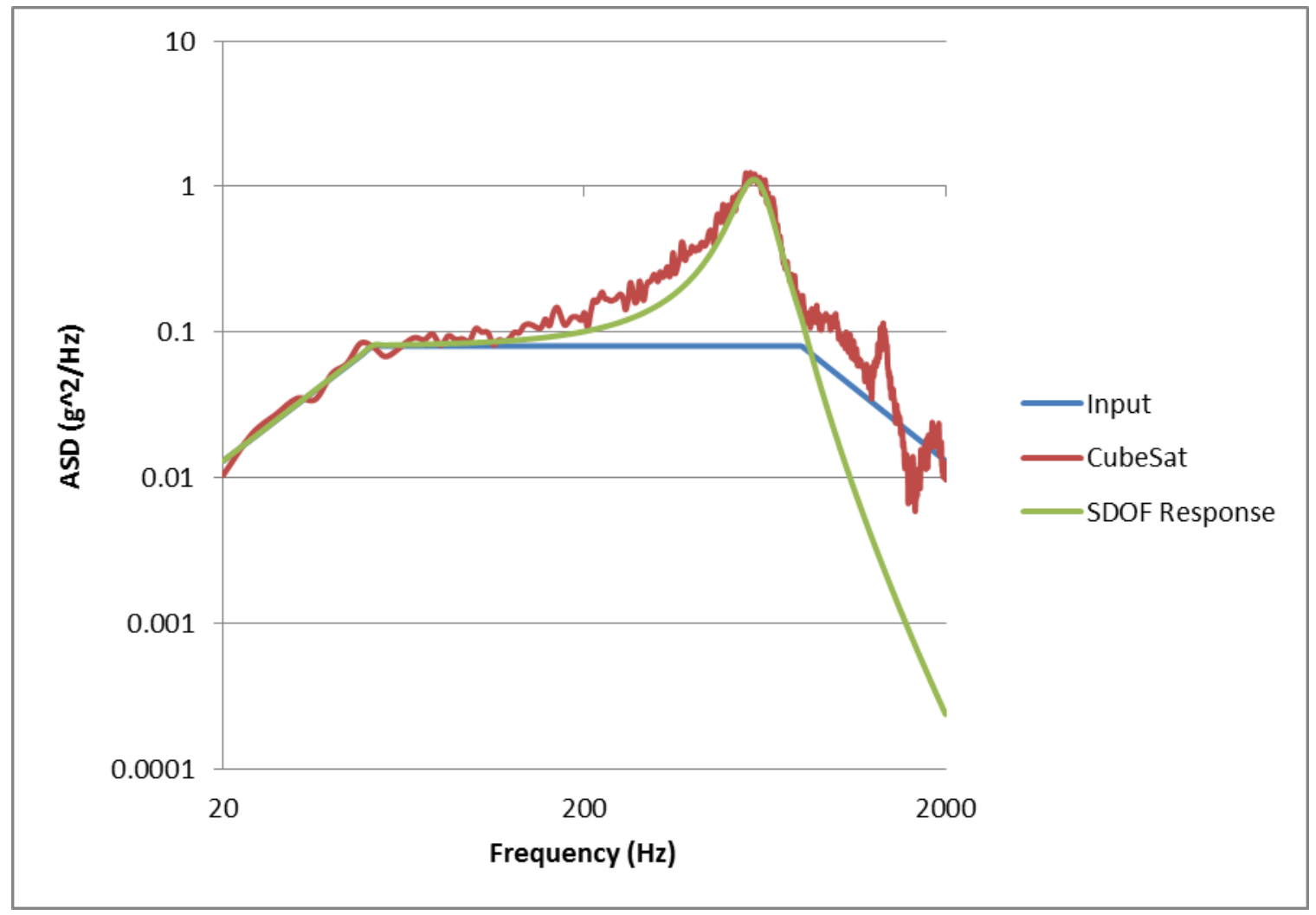

Figure 56: Fixed CubeSat response compared to SDOF model $\left(\omega_{n}=600 \zeta=0.12\right)$ in $X$ axis of a 1U TestPOD (test number 8-1). 
Just like with the $\mathrm{Z}$ axis, the SDOF model accurately approximates the CubeSat response up to the first peak. It also verifies there is no other transfer function between the CubeSat and deployer, and that the friction from the spring plungers is indeed fixing the CubeSat inside the deployer.

If the CubeSat is unconstrained, it can be modeled like an isolator is placed between the CubeSat and the deployer. Consider the same test setup from Figure 56, but with the spring plungers backed out so the CubeSat is allowed to translate. Figure 57 shows a SDOF isolator approximation for the response of an unfixed CubeSat in the $\mathrm{X}$ axis of the $1 \mathrm{U}$ TestPOD. 


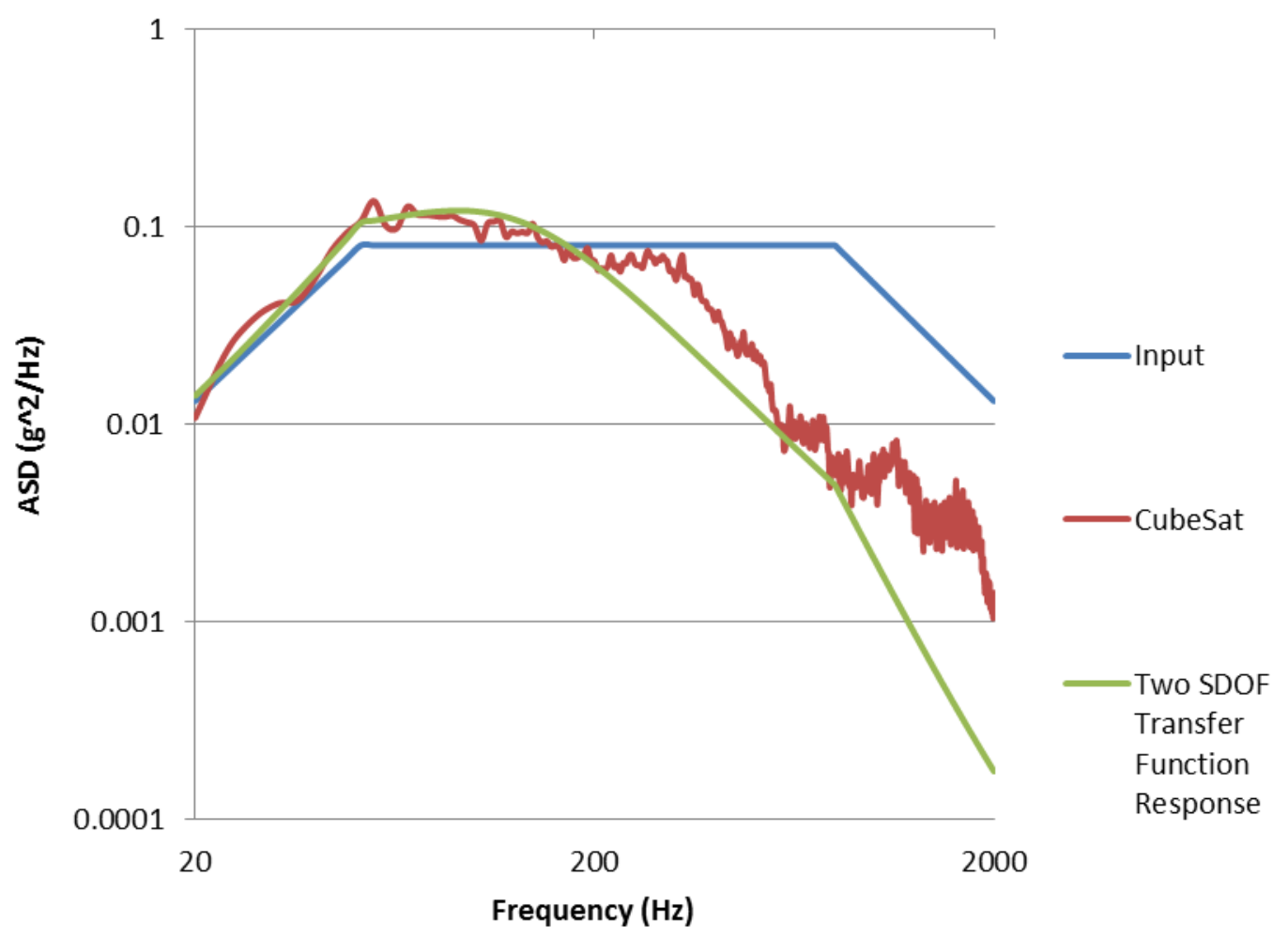

Figure 57: Unconstrained CubeSat response compared to SDOF isolator model $\left(\omega_{n}=120 \zeta=.8\right)$ in $\mathrm{X}$ axis of a 1U TestPOD (test number 9-1).

The isolator model can provide a good approximation of the CubeSat response inside the deployer if good estimates of the isolator natural frequency and damping are chosen. The natural frequency is a function of how much the CubeSat can displace inside the deployer and the damping ratio is primarily a function of inelastic collisions between the CubeSat and deployer. These values would have to be characterized experimentally. Because the CubeSat rattling inside the deployer acts as a particle damper, the damping values for the isolator transfer function are quite high. 
The SDOF isolator approximation is more accurate in the lower frequencies and begins to deviate from experimental data in the higher frequencies. This is because the deployer dynamics where not yet added this model. Only using the SDOF isolator without deployer dynamics is still a good approximation because the CubeSat is largely isolated from deployer dynamics.

To improve the accuracy of the model by including the deployer dynamics, the deployer dynamics transfer function must be added. Because the SDOF 1U TestPOD FRF does not couple with the SDOF isolator model, the two transfer functions can be multiplied together. While the two transfer functions do not couple, the damping ratio of the deployer must be increased because the unconstrained CubeSat now acts as a particle damper. Figure 58 shows how the deployer's FRF and the isolator FRF can be multiplied together to approximate CubeSat response in the $\mathrm{X}$ axis of the $1 \mathrm{U}$ TestPOD. The deployer's FRF is equivalent to the FRF in Figure 56, but the damping ratio is increased to 0.5 . The SDOF isolator FRF is the same as the one from Figure 57. 


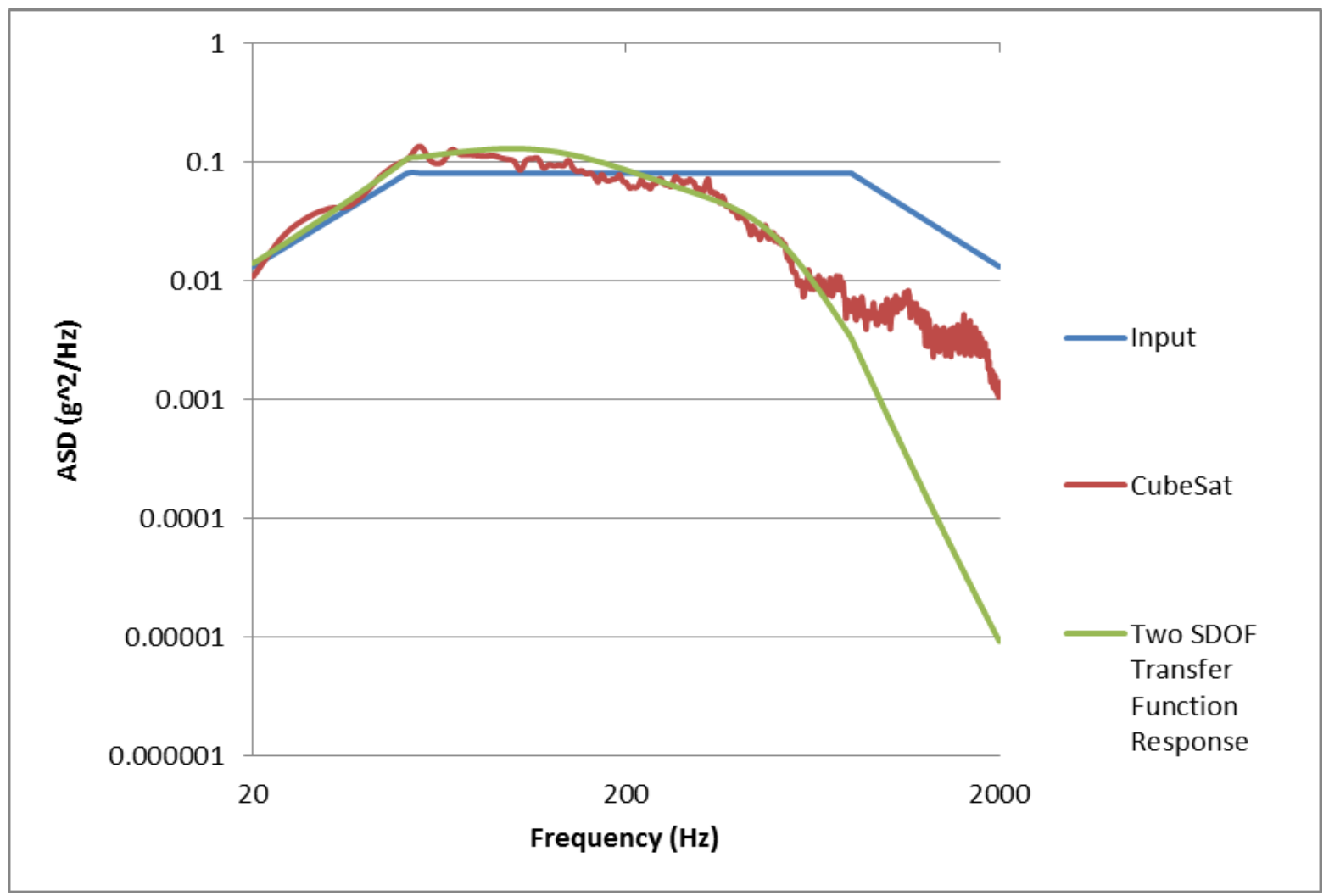

Figure 58: Two SDOF FRFs used to approximate the CubeSat response in the $X$ axis of the $1 \mathrm{U}$ TestPOD.

Including the deployer's dynamics makes the model more accurate. The theoretical response still underestimates the response after the deployer's first peak because SDOF deployer response was assumed. If a MDOF model was used for the deployer, the model would obtain even more accurate results than a SDOF FRF. For MDOF analysis, isolator "cbush" elements can be used between the deployer and CubeSat in the Finite Element Model (FEM) during random response analysis to simulate a CubeSat that has settled into its isolated position during a random vibration test. For static CubeSat loads, Miles' equation would have to be implemented with natural frequency and damping values from the isolator FRF, not the deployer's FRF. 
These SDOF approximations provide analytical validation of the $\mathrm{X}$ and $\mathrm{Y}$ axes transfer functions. They show that CubeSats are fixed in the deployer when the spring plunger friction is high enough, and that the CubeSats are isolated from the deployer when the CubeSats can translate. The coherence between the SDOF isolator model and actual data show the settled unfixed CubeSat is truly being isolated in a relatively predictable manner.

\section{Modeling CubeSat Response in $X$ and $Y$ Axes}

Transfer functions that provide approximations of CubeSat response were developed for CubeSats that are fixed inside the deployer and for CubeSats that are allowed to translate. However, experimental data has shown that CubeSats can start fixed inside a deployer and become isolated during the test. This means the model can change during a random vibration test or during launch. These models can be used, however, to approximate the CubeSat response at the beginning of the test and once the response has reached steady state.

Generally, at the beginning of CubeSat tests, the CubeSat is at least partially fixed inside the deployer. Figure 59 and Figure 60 show the SDOF deployer approximation for $\mathrm{X}$ axis CubeSat response inside the $3 \mathrm{U}$ TestPOD and P-POD for the beginning of the test. 


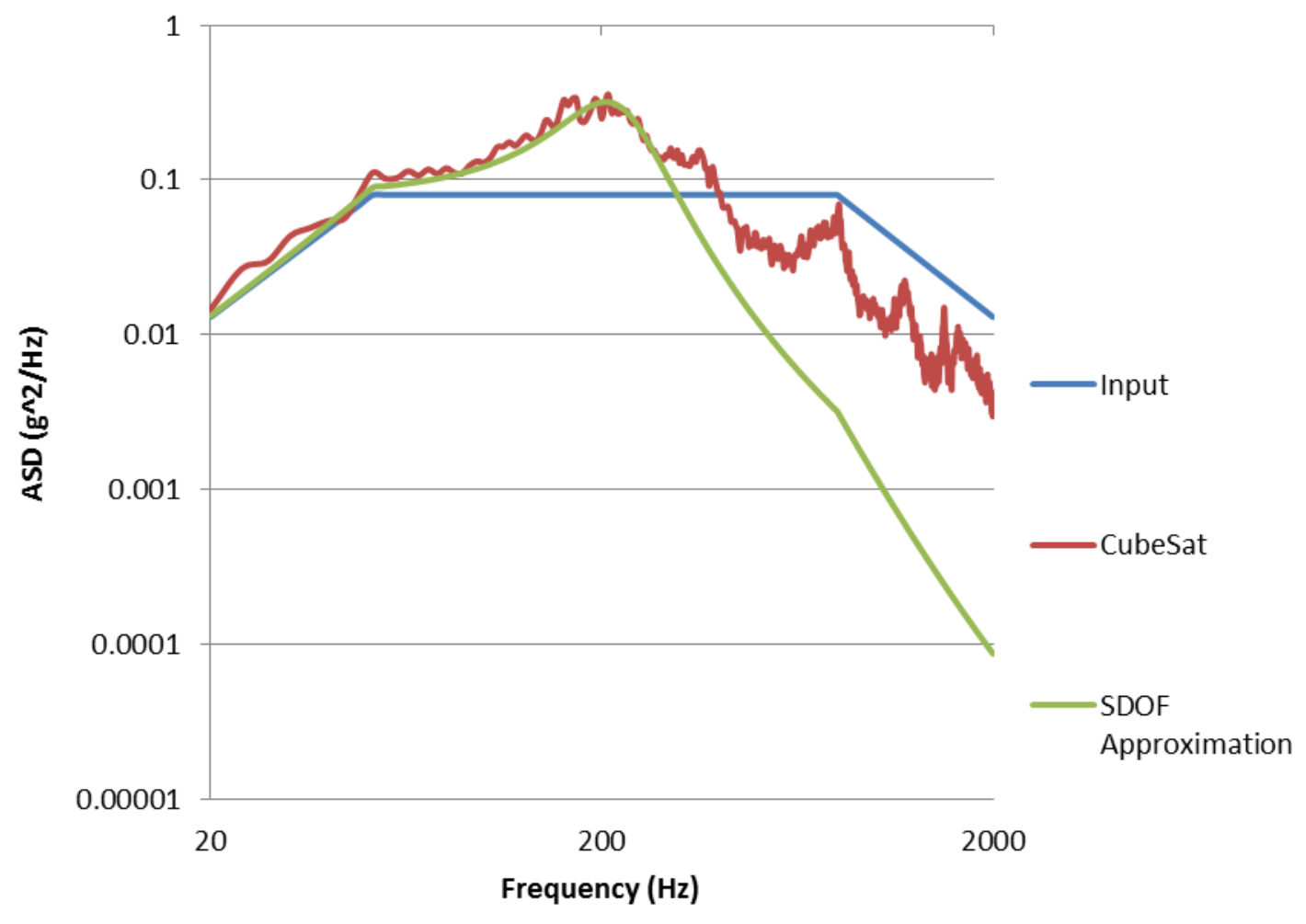

Figure 59: CubeSat response at beginning of test in 3U TestPOD compared to SDOF deployer model $\left(\omega_{n}=220 \zeta=.3\right)($ test number 15-2). 


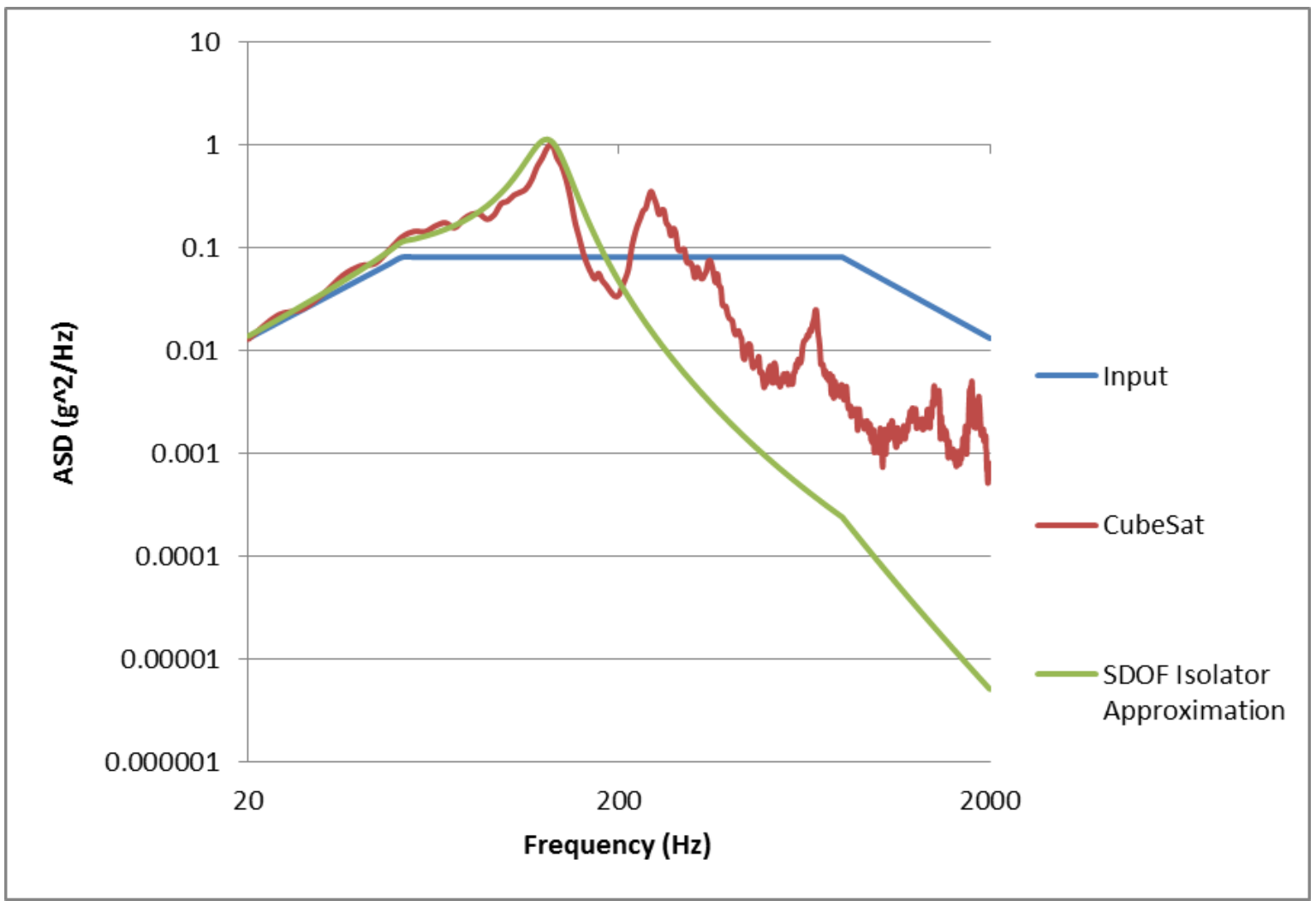

Figure 60: CubeSat response at beginning of test in P-POD compared to SDOF deployer model $\left(\omega_{n}=130 \zeta=.14\right)($ test number 22-2).

Just as with all SDOF approximations of fixed CubeSat response, the theoretical response underestimates the actual response for frequencies after the first peak. And just like with previous analysis, this can be fixed by using a MDOF FRF instead of a SDOF FRF.

Figure 59 and Figure 60 further validate that the CubeSat is fixed inside the deployer at the beginning of the tests. The data from the approximation also show that the damping ratios are consistently higher for the $\mathrm{X}$ axis than the $\mathrm{Z}$ axis. This is likely due to the fact that, even though the friction from the spring plungers largely constrains the CubeSat at the beginning of the test, the CubeSat is still allowed to translate slightly. 
The friction from the translation and the impacts of the CubeSat with the deployer rails adds damping to the system, explaining why the damping ratio is higher in the $\mathrm{X}$ axis than the $\mathrm{Z}$ axis.

As the tests progress, the CubeSats become unconstrained and are allowed to translate in the $\mathrm{X}$ axis. The isolation effect is seen, and CubeSat response can be approximated with a SDOF isolator. This is shown in Figure 61 and Figure 62, where a theoretical SDOF isolator is used to approximate the response of CubeSats in the $3 \mathrm{U}$ TestPOD and P-POD after about 20 seconds of testing.

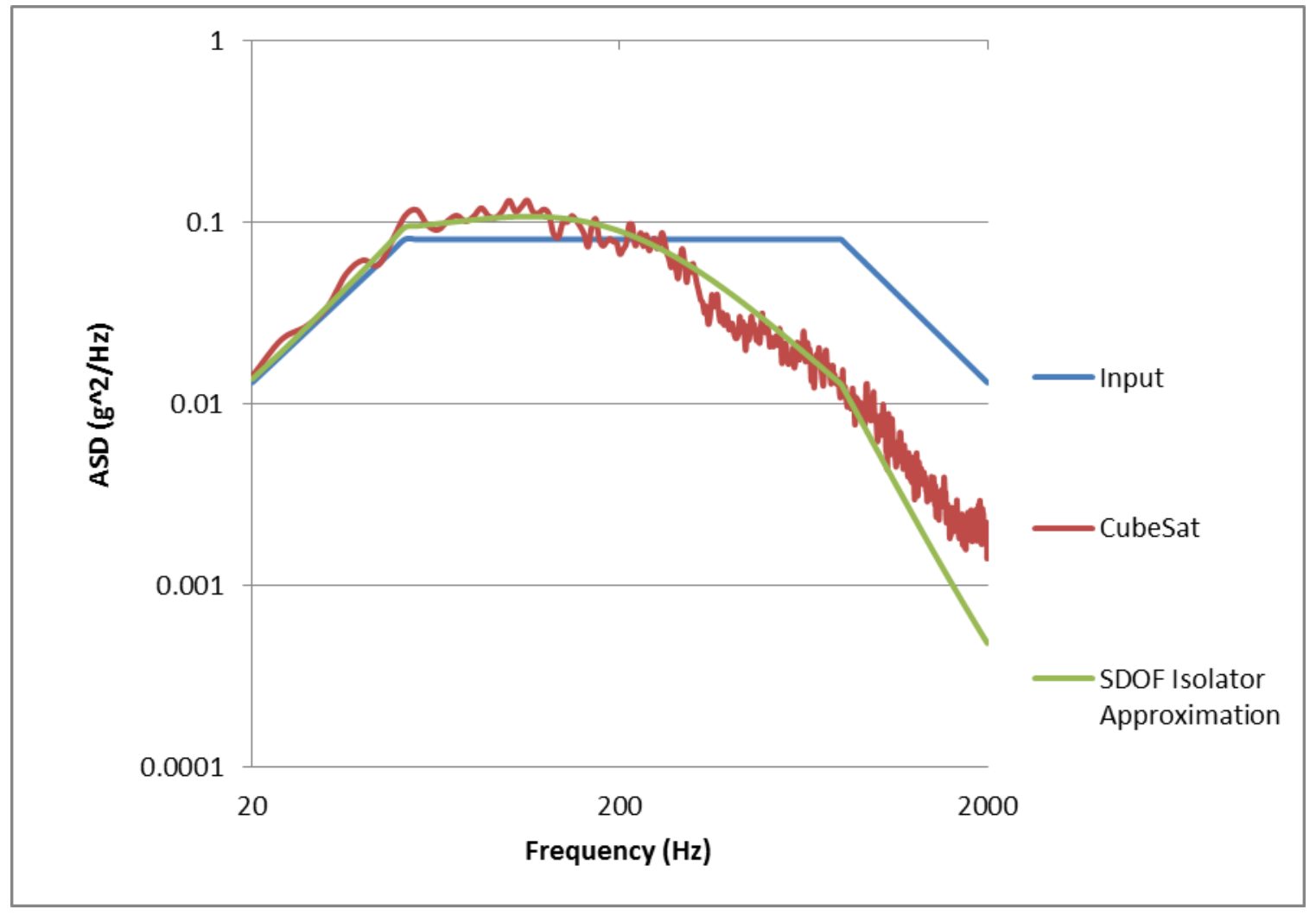

Figure 61: CubeSat response at end of test in $\mathrm{X}$ axis of $3 \mathrm{U}$ TestPOD compared to SDOF isolator model $\left(\omega_{n}=120 \zeta=1\right)($ test number 15-2). 


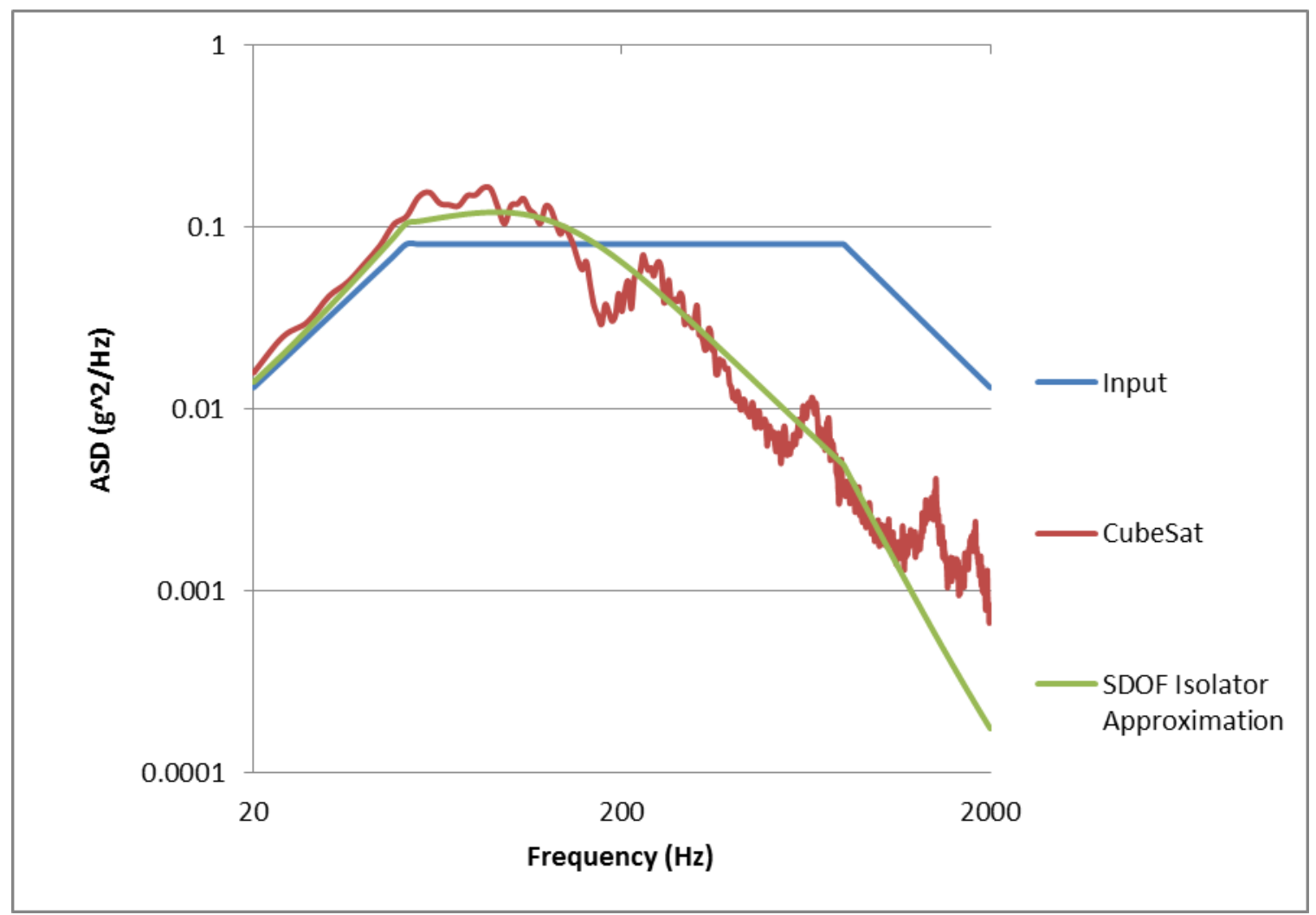

Figure 62: CubeSat response at end of test in $\mathrm{X}$ axis of P-POD compared to SDOF isolator model

$$
\left(\omega_{n}=120 \zeta=.8\right)(\text { test number 22-2). }
$$

This isolator model provides an approximate response for CubeSats after they have been exposed to a random vibration profile long enough to become settle into an isolated position.

\section{Vibration Model Conclusion}

Experimental data and analysis has shown that:

- CubeSat response can be modeled using standard linear methods in the $\mathrm{Z}$ axis 
- Transfer functions created from experimental data or FEA can be used to obtain the CubeSat's frequency response

○ Miles' equation can be used to approximate CubeSat static loads using estimates of the deployer's natural frequency and damping

- CubeSat response has two models in the $\mathrm{X}$ and $\mathrm{Y}$ axes, depending on if friction from the spring plungers fixes the CubeSats or not

- If the CubeSat is fixed, response can be modeled like the $\mathrm{Z}$ axis

- If the CubeSat is allowed to translate, response can be modeled as if a SDOF isolator was placed between the deployer and CubeSat

- Miles equation would then use SDOF isolator natural frequency and damping values

- CubeSats generally start fixed in the deployer, then become unconstrained in the $\mathrm{X}$ and $\mathrm{Y}$ axes

- Analysts should be aware that vibration loads from both models will be seen during testing and flight, and should consider using the worst case

- Frequency response parameters are needed to use the vibration models (resonant frequencies and damping ratios)

○ Can be obtained experimentally or through FEA

O Much harder to obtain in $\mathrm{X}$ and $\mathrm{Y}$ axes than the $\mathrm{Z}$ axis

- They were not characterized in this thesis 
The $\mathrm{Z}$ axis can be analyzed like any regular structure. Resonant frequency and damping information can be obtained through conventional methods and the frequency response from any profile can be obtained using standard linear analysis methods. Static loads can be approximated using Miles' equation with natural frequency and damping from the deployer.

There are two models for CubeSat response in the $\mathrm{X}$ and $\mathrm{Y}$ axes: one for when the CubeSat is fixed in the deployer by the friction from the spring plungers and one for when the CubeSat can translate. When the CubeSat is fixed inside the deployer, analysis can be performed in the same manner as the $\mathrm{Z}$ axis. When the CubeSat is allowed to translate, a SDOF isolator transfer function must be added in between the deployer and CubeSat. Isolator natural frequency and damping values (obtained empirically) must be used for Miles' equation.

The $\mathrm{X}$ and $\mathrm{Y}$ axes analysis is much more difficult than the $\mathrm{Z}$ axis. The CubeSat constraint in the $\mathrm{X}$ and $\mathrm{Y}$ axes is inconsistent and depends on many factors that can only be obtained empirically (normal force from spring plungers, friction coefficient, etc.). The constraint can even change in the middle of the test. This means the analyst often does not know what model to use for the CubeSat response, and must resort to worst case load scenarios. Furthermore, obtaining resonant frequency and damping information in the $\mathrm{X}$ and $\mathrm{Y}$ axes is much more difficult than the $\mathrm{Z}$ axis. The isolator frequency for isolated CubeSats can only be obtained through thorough characterization due to the nonlinear canister constraint. While the damping ratios in the $\mathrm{Z}$ axis are around what is 
generally seen in metallic structures, the damping ratio for both fixed and unfixed CubeSats in the $\mathrm{X}$ and $\mathrm{Y}$ axes vary widely due to damping from impacts and friction.

\section{Effect of Deployer Design on CubeSat Loads}

Deployer Design's Effect in the Z Axis

The deployer dynamics affect the CubeSat in the $\mathrm{Z}$ axis because the CubeSat is fixed by the spring plungers. This means that the CubeSat frequency response will change in deployers with different structures. Figure 63 shows the CubeSat response in the $1 \mathrm{U}$ TestPOD, $3 \mathrm{U}$ TestPOD and P-POD. All tests were run with $1 \mathrm{~kg}$ CubeSat mass models.

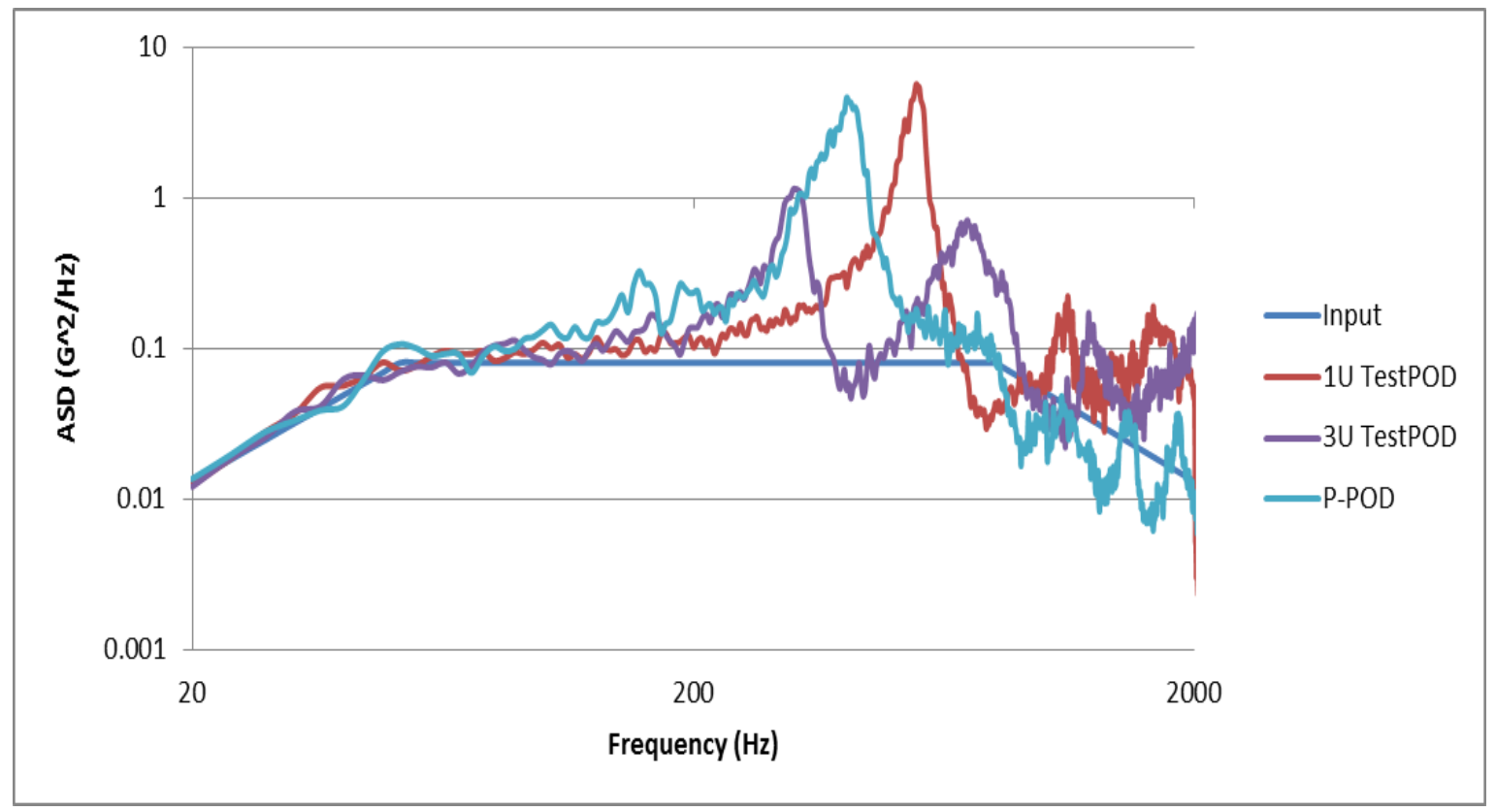

Figure 63: $\mathrm{Z}$ axis CubeSat response in $1 \mathrm{U}$ TestPOD, 3U TestPOD and P-POD (test numbers

\section{$3,12-2$, and 18-2).}


Figure 63 shows just how different the frequency response of a CubeSat can be inside different deployers. This is expected, as the CubeSat-deployer system can be modeled as a single structure in the $\mathrm{Z}$ axis, and all of these deployers are different structures.

Because CubeSats have different frequency responses in different deployers, the $\mathrm{G}_{\mathrm{rms}}$ for CubeSats in different deployers is different. The random profile from the tests in Figure 63 was GEVS acceptance at $9.99 \mathrm{G}_{\mathrm{rms}}$. The CubeSat mass model response was 23.33 $\mathrm{G}_{\mathrm{rms}}$ in the $1 \mathrm{U}$ TestPOD, $17.20 \mathrm{G}_{\mathrm{rms}}$ is the $3 \mathrm{U}$ TestPOD, and $19.01 \mathrm{G}_{\mathrm{rms}}$ in the PPOD. These results are summarized in Table 2.

Table 2: CubeSat response to GEVS acceptance profile in different deployers.

\begin{tabular}{|c|c|}
\hline Deployer & CubeSat Response to GEVS Acceptance $\left(\mathbf{G}_{\mathbf{r m s}}\right)$ \\
\hline 1U TestPOD & 23.33 \\
\hline 3U TestPOD & 17.20 \\
\hline P-POD & 19.01 \\
\hline
\end{tabular}

The $\mathrm{Z}$ axis CubeSat response in all of these deployers was much higher than the random vibration profile input. This is because the majority of the CubeSat's frequency response is in the amplification region of the deployer-CubeSat system's resonant peaks. There is not much of an isolation region for these deployers because they are MDOF structures with additional resonant peaks after the first peak (where SDOF structures only have a region of isolation after the resonant peak). The deployer's resonant peaks result 
in "deployer amplification" in the $\mathrm{Z}$ axis, which can lead to CubeSats seeing double the vibration input from the shake table or launch vehicle.

\section{Deployer Design's Effect in $X$ and $Y$ Axes}

In the $\mathrm{X}$ and $\mathrm{Y}$ axes, the CubeSats generally start fixed inside the deployer and become unconstrained as the test progresses. When the CubeSat is fixed inside the deployer, the deployer's dynamics greatly affect CubeSat loads, just like with the $\mathrm{Z}$ axis. When the CubeSat is allowed to translate, the CubeSat becomes isolated from the deployer, and the deployer's dynamics have less of an effect on CubeSat loads. 
Consider the $\mathrm{X}$ axis CubeSat response from the tests in the $1 \mathrm{U}$ TestPOD, $3 \mathrm{U}$ TestPOD and P-POD. At the beginning of the tests, the CubeSats are fixed in the deployer, and the deployer dynamics affect the CubeSat frequency response. Figure 64 shows the CubeSat responses inside these three deployers at the beginning of their tests.

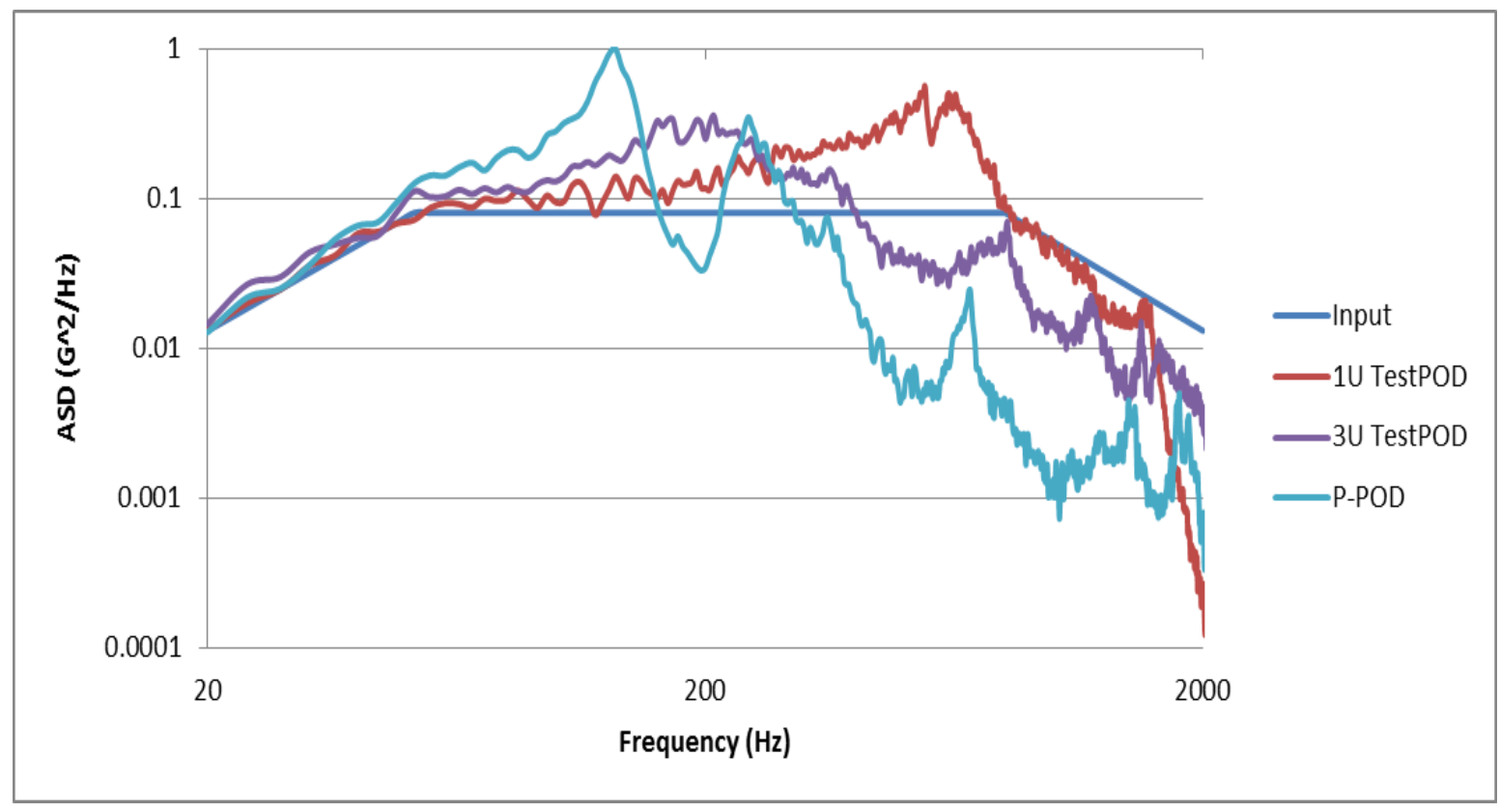

Figure 64: $\mathrm{X}$ axis CubeSat response in $1 \mathrm{U}$ TestPOD, 3U TestPOD and P-POD beginning of test (test numbers 7, 15-2 and 22-2).

It can be seen that the different deployer structures with different resonant peaks affect the CubeSat's frequency response. As the tests progress, the CubeSats become isolated from their deployers. Figure 65 shows that, once the CubeSats become isolated from the deployer, the difference in deployer structure has much less of an effect. The responses are similar even though they are in very different deployers. 


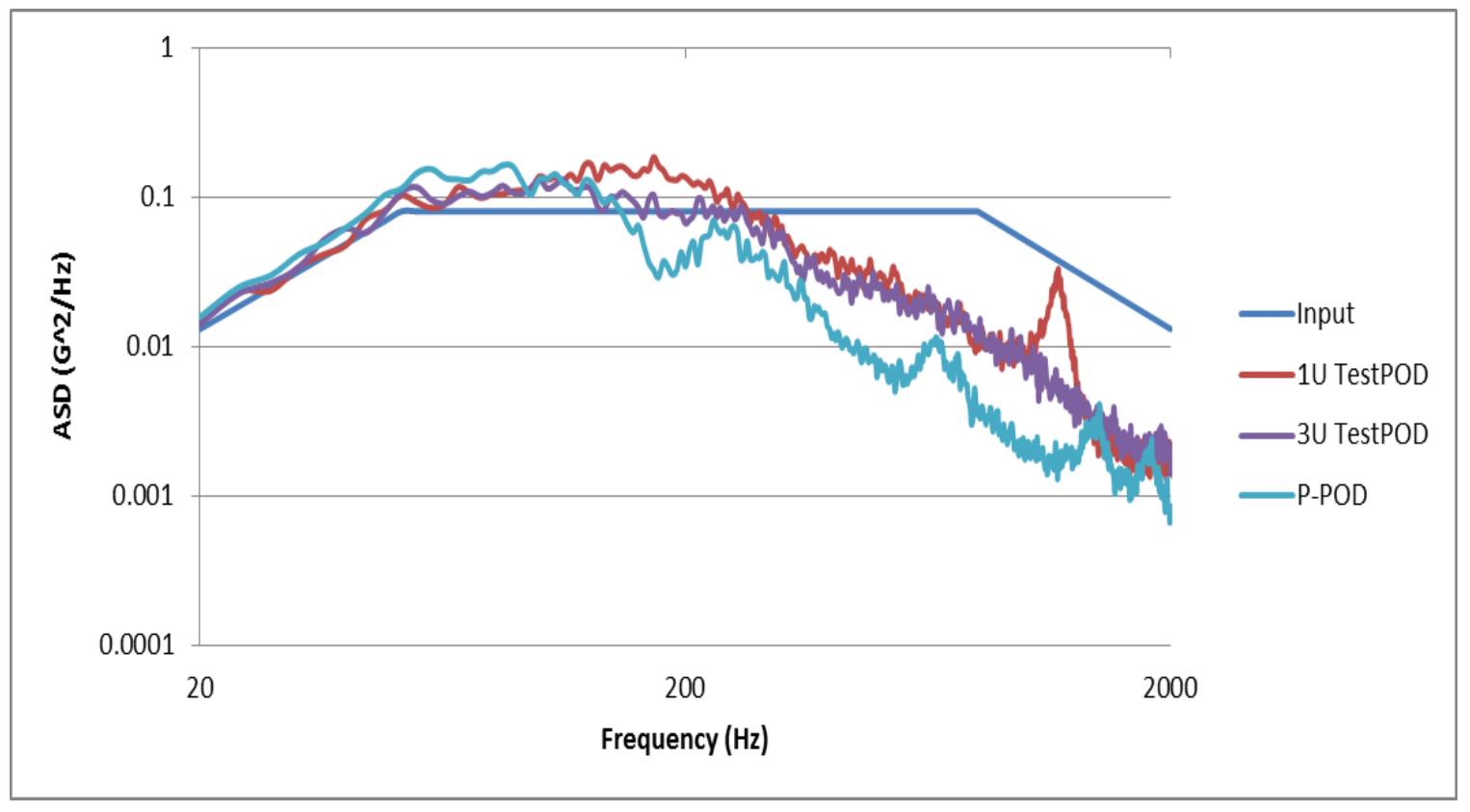

Figure 65: $\mathrm{X}$ axis CubeSat response in 1U TestPOD, 3U TestPOD and P-POD end of test (test numbers 7, 15-2 and 22-2).

Table 3 summarizes the $\mathrm{G}_{\mathrm{rms}}$ response for CubeSats in the three deployers at the beginning of the tests (fixed CubeSat) and end of the tests (isolated CubeSat).

Table 3: CubeSat response to GEVS random vibration profile for fixed (beginning of test) and isolated (end of test) CubeSats in different deployers.

\begin{tabular}{|c|c|c|}
\hline Deployer & Fixed CubeSat Response $\left(\mathbf{G}_{\mathbf{r m s}}\right)$ & $\begin{array}{c}\text { Isolated CubeSat } \\
\text { Response }\left(\mathbf{G}_{\mathbf{r m s}}\right)\end{array}$ \\
\hline 1U TestPOD & 14.21 & 7.23 \\
\hline 3U TestPOD & 9.76 & 6.38 \\
\hline P-POD & 8.32 & 5.35 \\
\hline
\end{tabular}


This data shows that, when a CubeSat is fixed in the deployer, different deployer structures greatly change CubeSat response. When the CubeSat is isolated, the deployer's dynamics have less of an effect on the CubeSat. The $\mathrm{G}_{\mathrm{rms}}$ response for the CubeSat in the 1U TestPOD was 70.8\% higher than the CubeSat response in the P-POD at the beginning of the test (when they were fixed in the deployer). However, when the CubeSats were isolated at the end of the tests, CubeSat response in the 1U TestPOD was only $35.1 \%$ higher than the P-POD.

Another important point this data illustrates is that CubeSat response is much lower when the CubeSat is isolated. Deployer amplification is avoided when the CubeSats are isolated from the deployer's resonant peaks. The response was lower for the isolated CubeSats compared to the constrained CubeSats in the 1U TestPOD, 3U TestPOD and P-POD.

\section{Conclusions on Deployer Design}

- When CubeSats are fixed, the deployer structure and its resonant frequencies have a great effect on CubeSat response

○ This can result in "deployer amplification" depending on what frequencies the peaks are at and how many peaks there are

- When CubeSats are allowed to translate, the deployer structure has much less of an effect on the CubeSat response

- CubeSats in all deployers tend to display about the same SDOF isolator response when allowed to translate

- CubeSats that are allowed to translate have lower loads than fixed CubeSats 
○ CubeSats are isolated from deployer amplification

When CubeSats are fixed in their deployers, the structure of the deployer plays a large role in the CubeSats frequency response. Because the CubeSats resonate with the deployer, these loads can be amplified from the table input. When the CubeSats are allowed to translate, deployer dynamics have much less of an effect. Because the CubeSats are isolated from deployer resonance, CubeSat see lower loads when allowed to translate compared to when they are fixed.

It should be noted that data was only taken from 20-2000 Hz. CubeSats impact the rails when they are allowed to translate, and this could create shocks or high frequency impulses that are above the accelerometer limits used in this thesis. When it is stated that loads are less for CubeSats that are allowed to translate, this is implying loads that can be measured at frequencies between $20-2000 \mathrm{~Hz}$. 


\section{CHAPTER 5: Design Concept for Future P-POD}

Random vibration profiles for deployers can be very high, in some cases over 30 $\mathrm{G}_{\mathrm{rms}}$. These high loads can lead to CubeSat failures and discourages growth in the CubeSat community. Furthermore, the current P-POD utilizes a canisterized constraint of CubeSats, as do many other deployers. As shown earlier in this thesis, the canisterized constraint can make modeling the vibration environment difficult. This chapter proposes a concept for a new P-POD design that lends itself to simpler vibration analysis while also reducing loads from random vibration inputs.

\section{P-POD Design Trades}

\section{CubeSat Constraint inside P-POD}

To design a P-POD that lends itself to linear vibration analysis, the new P-POD design must constrain the CubeSat in a more consistent manner than the current canisterized constraint. While the constraint in the $\mathrm{Z}$ axis is predictable, CubeSats in the $\mathrm{X}$ and $\mathrm{Y}$ axes can be fixed or allowed to translate depending on many factors. This inconsistency means that changes in random vibration profile or CubeSat mass can completely change the vibration model used. A method of constraining CubeSats inside the P-POD must be created that keeps the constraint consistent for any random vibration profile or CubeSat design.

One method of creating a constraint that lends itself to vibration analysis is completely fixing the CubeSat in all axes. By fixing the CubeSat inside the deployer, the entire system can be modeled as one continuous structure. This leads to more consistent 
analysis methods with constraints that are not dependent on the vibration load (as with the $\mathrm{X}$ and $\mathrm{Y}$ axes of the P-POD). If the CubeSat is completely fixed in the deployer in all axes, the entire CubeSat-deployer system can be modeled like the P-POD's Z axis.

Another method to consistently constrain CubeSats inside the P-POD is to allow the CubeSat to translate in all three axes instead of just X and Y. Because the CubeSat would be able to translate in the $\mathrm{Z}$ axis, there would be no friction in the $\mathrm{X}$ and $\mathrm{Y}$ axes. This means there will be no slip load and the CubeSat will always be allowed to translate. This lets analyst model the entire system using the isolator model discussed in Chapter 4 without worrying about whether the CubeSat is fixed, isolated, or in transition. This constraint system is not truly linear, but, as shown earlier, can be approximate using linear vibration transfer functions.

By utilizing one of these methods, a more consistent CubeSat-deployer vibration model can be developed and characterized. This lets developers understand the loads their CubeSat will see for their mission. Having a CubeSat-deployer system that can be modeled is important for the future P-PODs, and this trade will have a large influence on the P-POD design concept introduced in this thesis.

\section{Vibration Mitigation}

With a better understanding of how loads are driven from the deployer to the CubeSat, a concept for a P-POD that mitigates vibration for CubeSats can be developed. Several methods of vibration mitigation are traded, included vibration damping and vibration isolation. While all methods could theoretically work, vibration isolation of the CubeSat from the deployer was the chosen method. 


\section{Damping Deployer}

When fixed in the deployer, the CubeSat response can be amplified by the deployer's resonant peaks. This amplification can be attenuated by adding damping to the deployer structure. Figure 66 shows an example of how damping would mitigate vibration loads from deployer amplification. The undamped case is from experimental data of a CubeSat in a standard 1U TestPOD. The damped response is the theoretical CubeSat response if the $1 \mathrm{U}$ TestPOD was damped.

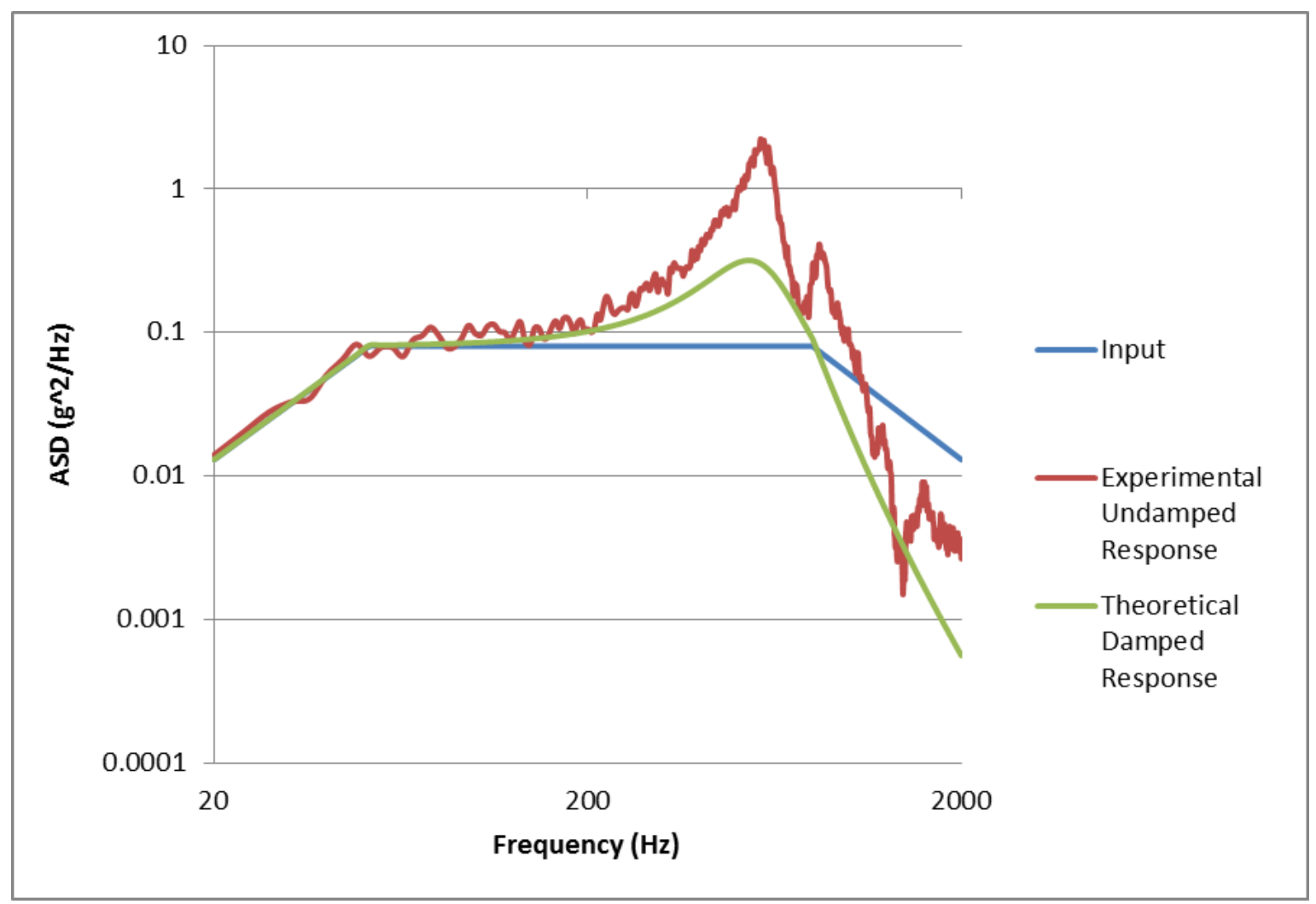

Figure 66: Example of how damping mitigates deployer amplification for CubeSats.

When a CubeSat is fixed inside the P-POD instead of the 1U TestPOD, there are multiple resonant peaks. If the P-POD structure were to be damped, all of the resonant peaks would be lower, reducing the amplification of the CubeSat at each peak. Any 
regions of isolation, however, would have increased transmissibility. Damping has no effect before the deployer's first resonant peak.

When the CubeSat is not fixed in the P-POD (the general case for the $\mathrm{X}$ and $\mathrm{Y}$ axes of the P-POD), damping of the structure would have a negligible effect. Damping only helps mitigate amplification at the deployer's resonant peaks. Because the CubeSat is isolated from deployer resonance when it is allowed to translate, damping is only effective when the CubeSat is fixed in the deployer.

There are several ways to add damping to the structure. A high damping material (such as viscoelastic rubber) could be sandwiched inside the deployer's structure. A particle damper could be attached to the top of the P-POD to increase the system's damping as well. A tuned mass damper could even be set to the deployer's resonant peaks and attached to the top of the P-POD's structure. While all of these methods could theoretically reduce the effect of deployer amplification, the reduction in vibration is probably not worth the cost and difficulty of implementation

\section{Isolating Deployer from Launch Vehicle}

Isolating the deployer from the launch vehicle is done by placing isolators at the deployer-launch vehicle interface. This lowers the resonant frequencies of the deployer, creating much larger regions of isolation. Figure 67 shows a very simplified example of how this increased region of isolation mitigates the effect of amplification at the deployer peaks. 


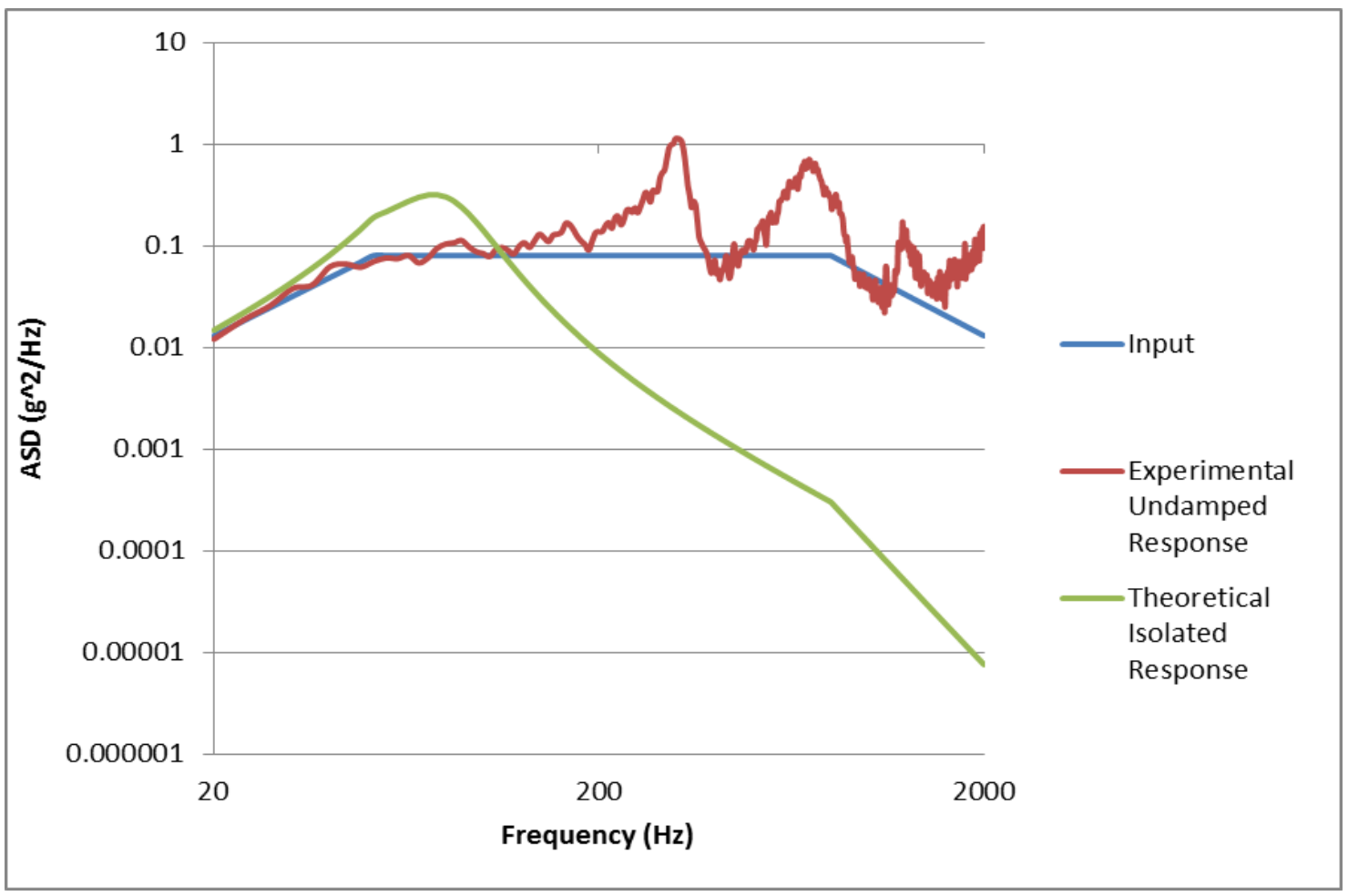

Figure 67: Example of how isolation could mitigate vibration loads.

The CubeSat response is isolated from vibration input at the frequencies of the deployer's resonant peaks when isolators are used. This mitigates the effect of deployer amplification. However, a new low frequency peak is introduced from the isolator resonant frequency.

Isolating the deployer from the launch vehicle can be very effective, and has been done for several CubeSat missions. However, isolators increase the dynamic envelope of the deployer. P-PODs are manifested on many launch vehicles in locations where any increase in dynamic envelope is unacceptable (see Appendix B). Examples of this include mission using adapters such as NPS CuL or CubeStack. It is also important 
to note the launch vehicle providers have a frequency requirement for the first mode of the P-POD, making isolators ineffective in many cases.

\section{Isolating CubeSat from Deployer}

Another method of vibration isolation is isolating the CubeSats from the deployer (as opposed isolating the deployer from the launch vehicle). This effect is seen when the CubeSat is allowed to translate inside the deployer, as with the $\mathrm{X}$ and $\mathrm{Y}$ axes of the $\mathrm{P}$ POD. When allowed to translate, the CubeSats are isolated from amplification due to deployer resonance. This effect can be replicated by constraining the CubeSat in the PPOD with a low stiffness material, creating an isolator between the CubeSat and the deployer.

Consider the $\mathrm{Z}$ axis vibration tests with CubeSats in the $1 \mathrm{U}$ TestPOD. When the spring plungers were dialed in and the CubeSat was fixed inside the 1U TestPOD, the CubeSat response was amplified at the deployer's resonance. However, when the spring plungers were backed out and the CubeSat was allowed to translate in the $\mathrm{Z}$ axis, the CubeSat was isolated from the deployer's peak and the overall vibration loads were reduced. Figure 68 shows the CubeSat responses from these cases, as well as the theoretical response of a CubeSat if an isolator were placed between the CubeSat and the deployer. 


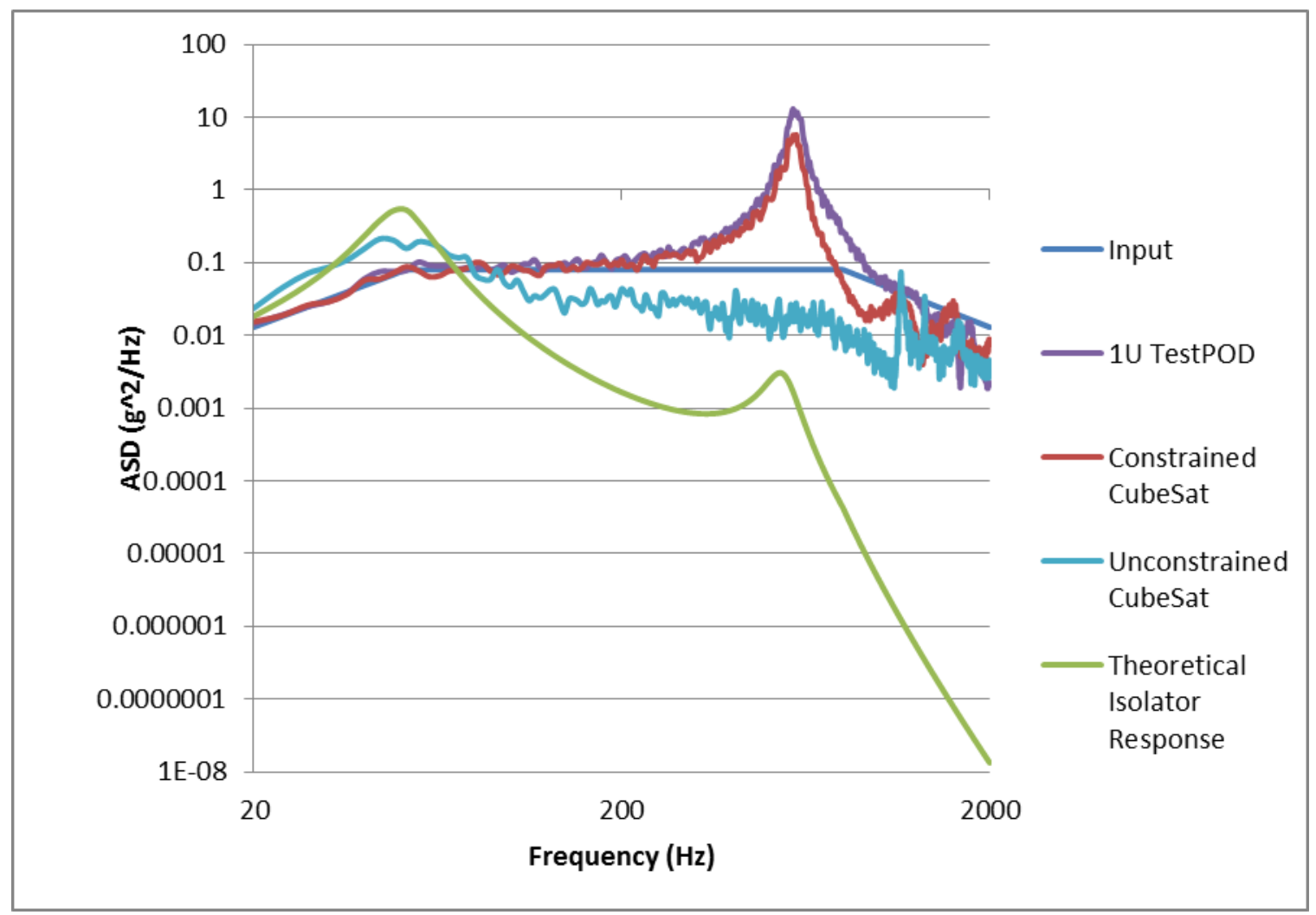

Figure 68: Comparison of fixed, unconstrained and isolated CubeSat response.

Notice that the constrained CubeSat resonates with the 1U TestPOD's resonant peak, but the unconstrained CubeSat does not. The theoretical isolator would have the same effect as allowing the CubeSat to translate.

Unlike isolating the deployer from the launch vehicle, isolating the CubeSat from the deployer does not increase the deployer's dynamic envelope. However, it does decrease the dynamic envelope for the CubeSat inside the deployer. A benefit of this method is the natural frequency of the deployer is not the isolator frequency. This means low isolator frequencies can be used while meeting launch vehicle frequency requirements. 


\section{P-POD Design Concept}

\section{Selected Constraint and Mitigation Trade}

Isolating the CubeSat from the deployer was the chosen method for vibration mitigation. By sandwiching a soft material in the rails, pusher plate, and door, the CubeSat can displace inside the deployer and be isolated from deployer resonance. The CubeSat will not be restricted in the $\mathrm{X}$ and $\mathrm{Y}$ axes by friction because there will be a negligible normal force in the $\mathrm{Z}$ axis. This creates a consistent constraint that lends itself to vibration analysis. There will still be a gap in the $\mathrm{X}$ and $\mathrm{Y}$ axes so CubeSat deployment will be unaffected. Figure 69 shows a schematic of how the CubeSat will be constrained.

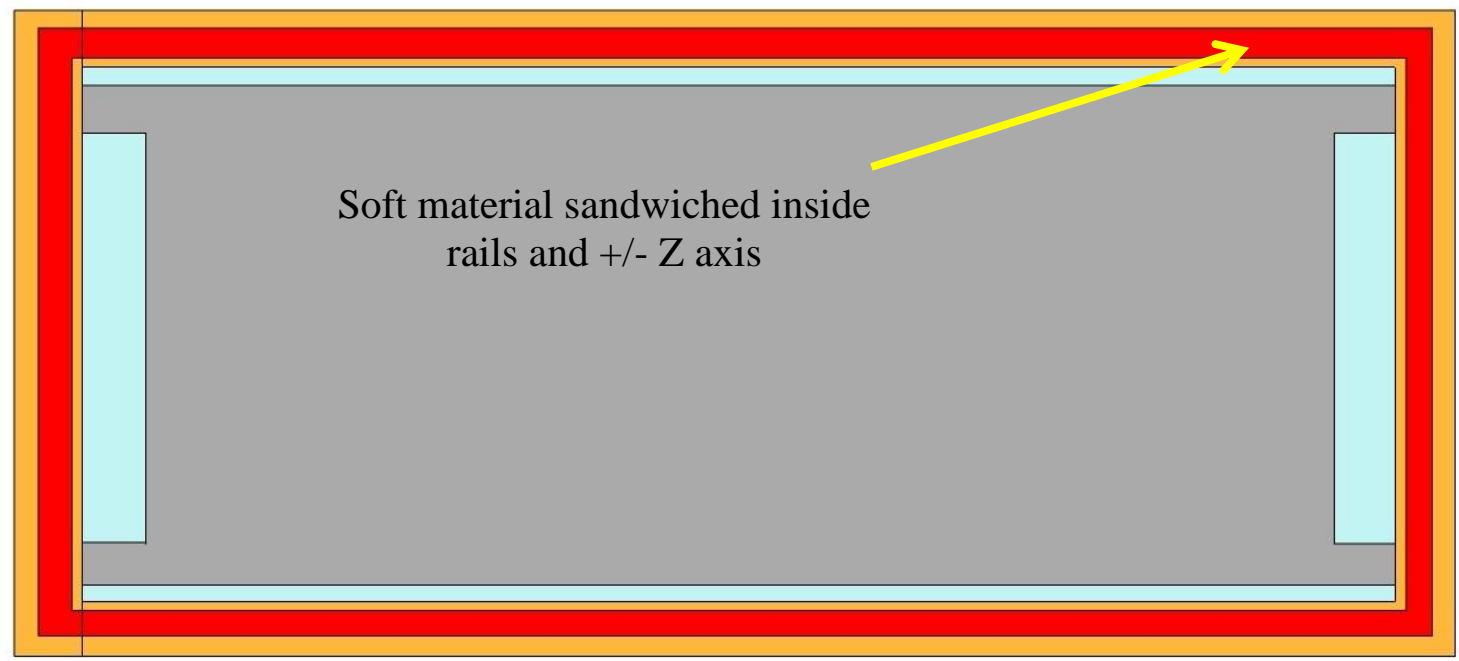

Figure 69: Schematic of concept for constraining CubeSat in future deployer.

\section{Isolator Frequency}

The isolator frequency will depend on the mission specific random vibration profile and deployer dynamics. For many random vibration profiles, lower isolator 
frequencies are more ideal. Lower isolator frequencies mean the region of isolation would start at a lower frequency, increasing the effectiveness of the isolators. However, some random vibration profiles have peaks in the low frequencies. The isolator frequency must be chosen to avoid setting the amplification region of the isolator at any low frequency peaks. To avoid coupling between the isolator and deployer resonant peaks, the isolator frequency must be at least one octave below the first mode of the deployer.

The isolator frequency can be tailored by varying the stiffness of the isolator material. Because of the canister constraint, there is no coupling between the $\mathrm{X}, \mathrm{Y}$ and $\mathrm{Z}$ axes. This means each axis can have its own isolator frequency that best suits the profile and deployer dynamics in that axis.

\section{Deployer Design}

\section{Isolator System}

Isolator material will be sandwiched in the rails, pusher plate and door. This sandwich lowers the stiffness of the rails, pusher plate and door while keeping the metal to metal contact between the CubeSat and deployer. Because the isolator frequency is mission specific, it is highly recommended that the isolators be removable so they can be installed late in the deployer manufacturing process. This requires the rails in the deployer to be removable and replaceable. It also requires easily removable plates with the isolator material sandwich to be attached on the door and pusher plate for the $\mathrm{Z}$ axis isolators. 


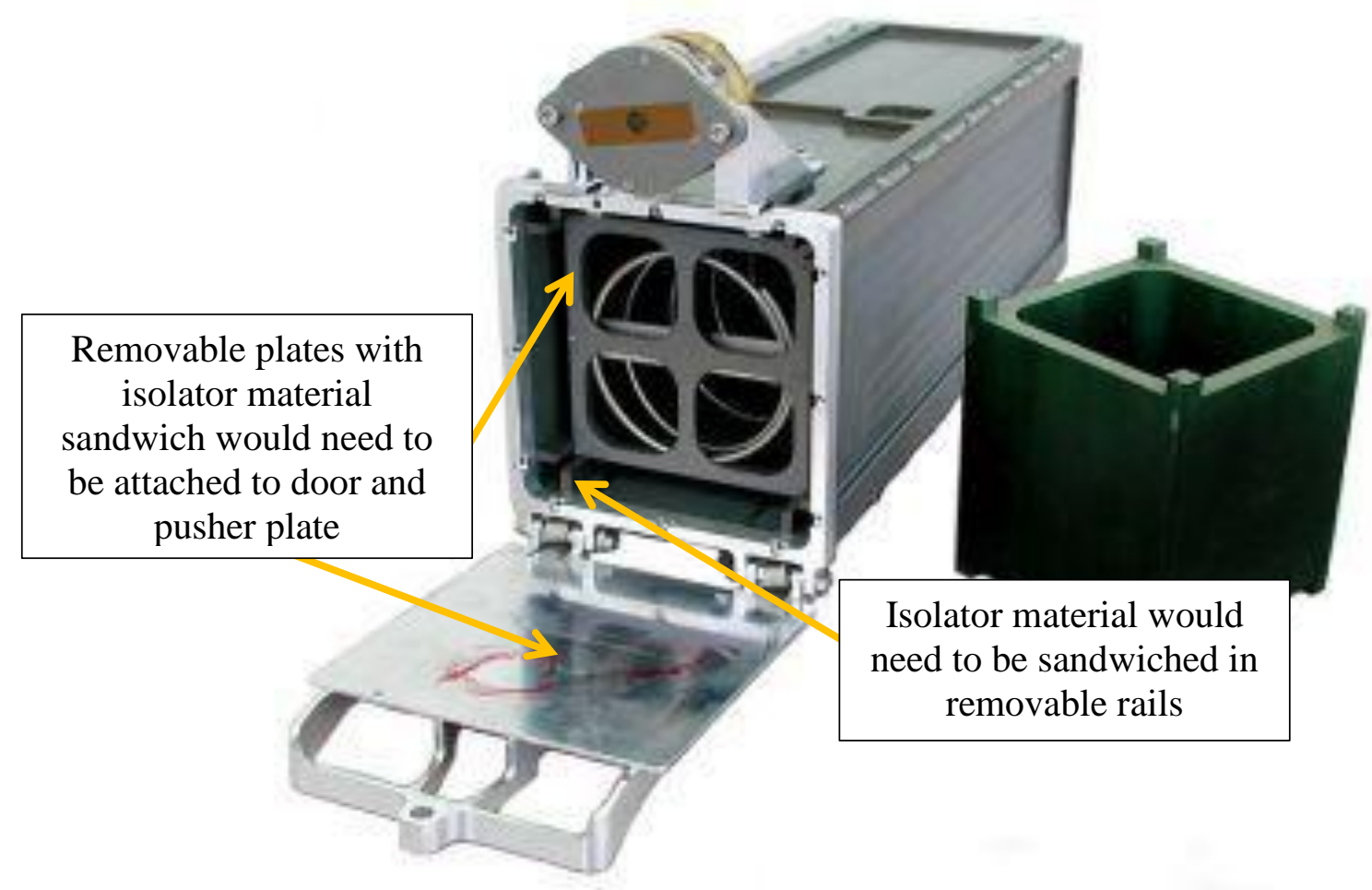

Figure 70: Location where removable rails and isolator plates would be in future deployer.

While there are many options for isolators to lower the stiffness between the CubeSat and deployer, any type of space grade rubber or foam would offer the most compact solution. Silicon foam has large temperature ranges and low outgassing properties, and is an ideal candidate for the isolator material. It is important to note that the material will be only deflecting in compression, so a rubber or foam will be nonlinear (a common issue with isolators).

\section{Deployer Resonant Frequencies}

It is important to ensure the deployer is stiff enough to allow a large enough range of isolator frequencies. If the deployer's first mode is too low, the range of usable isolator frequencies would be very small. Stiffer deployers would allow higher isolator 
frequencies, which could be necessary if there are low frequency peaks in a mission's random vibration profile. A first mode of at least $200 \mathrm{~Hz}$ is recommended.

\section{Mission Implementation}

\section{Characterization of Isolator Systems}

Characterization of the isolator system is required to obtain the desired isolator frequency. Several isolator systems with different stiffnesses must be characterized. Characterization would consist of vibration tests with varying masses of CubeSats for each isolator system. From these tests, charts of isolator frequency versus mass can be created. Figure 71 shows an example of what one of these characterization charts would look like.

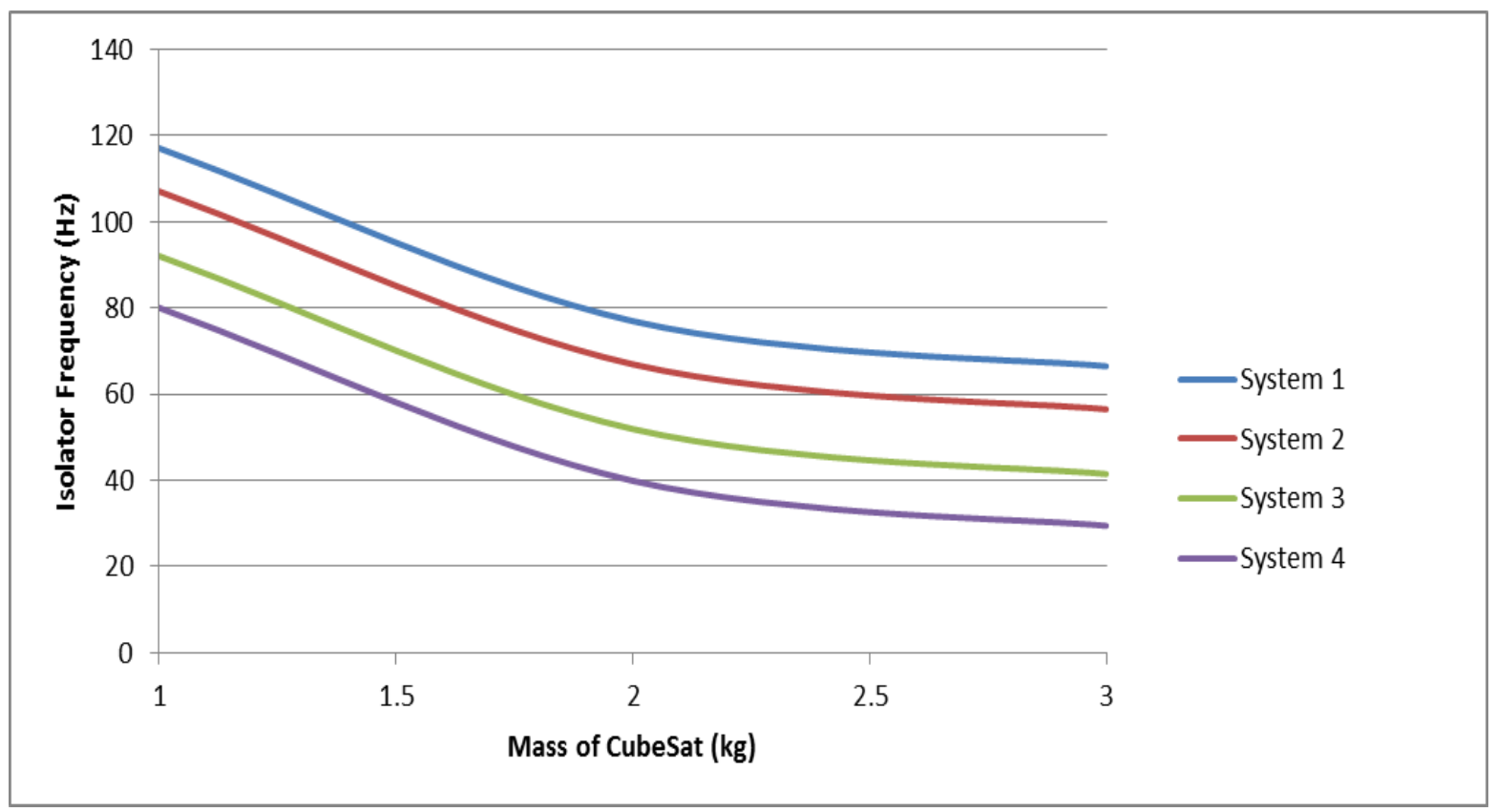

Figure 71: Example of isolation system characterization chart. 


\section{Choose Mission Specific Isolator System}

Given a mission specific random vibration profile, an isolator frequency can be chosen to best reduce loads. Once this frequency is chosen, the mass of the CubeSat can be used with the characterization charts to determine which characterized isolator system should be used on the deployer to obtain the desired isolator frequency. Because the isolator systems are removable, this system can be added late in the deployer manufacturing process. Acceptance or workmanship testing is required for all flight CubeSats and deployers. During this, validation tests can be run to ensure the isolator frequency is correct for the deployer, no coupling occurs between the deployer and CubeSat, and the isolators are effectively reducing loads.

It is important to note that there are static loads during launch that could affect the isolator. Because these isolator systems will likely be non-linear, the static acceleration offset can affect the isolator frequency. This effect is hard to reproduce experimentally on the ground and analysis will need to be performed to ensure the isolators perform correctly. 
CHAPTER 6: Proof of Concept for Isolation at Deployer Rail Interface

Goal of Isolator Test and Analysis

The goal of this chapter is to prove the P-POD design concept proposed in the last chapter is a feasible. To do this, a model of the proposed isolator constraint must be developed and tested. The design is feasible if the isolator constraint model:

- Mitigates vibration loads for CubeSats from random vibration profile

- Constrains the CubeSat in a manner that can be analyzed with linear vibration transfer functions

Satisfactory mitigation of vibration loads will be defined by ensuring the input random vibration profile is not amplified. For the constraint to be considered analyzable with linear transfer functions, it must be shown that a linear SDOF model can provide an approximation of the CubeSat frequency response.

The model will also have to be a good representation of a design that can be implemented into a future deployer. For the model to be considered implementable to future designs, it must use an isolator material that represents the properties of a space grade material that would be used for the flight version. The isolators must also use approximately the same volume and mass that would be implemented into the new design, and have the approximately the same dynamic envelope. 


\section{Test Setup}

\section{Isolator Model}

The Test CubeSat was used to model CubeSats isolated from the deployer. $1 / 8^{\text {th }}$ inch isolator material was placed on the rails of the $\mathrm{X}$ and $\mathrm{Z}$ axes of the Test CubeSat. The isolator material was placed on the CubeSat instead of the deployer because it was much easier to manufacture a CubeSat with isolators. The Test CubeSat with isolator rails is an acceptable model for analyzing isolated CubeSat response because the material, stiffness and dynamic envelope are similar to what would be implemented on the flight P-POD. Figure 72 shows the Test CubeSat with isolator material.

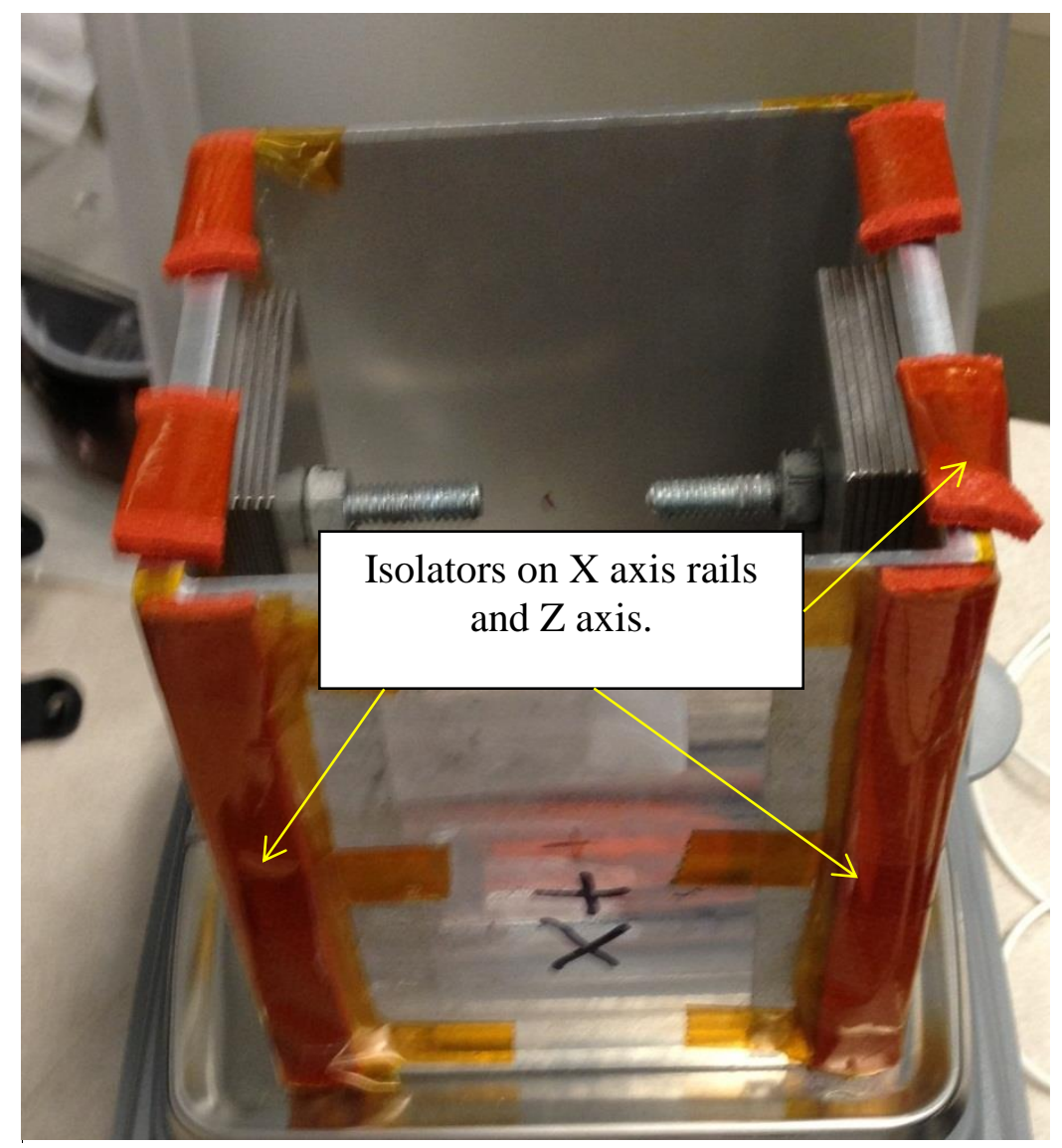

Figure 72: Test CubeSat with isolator rails.

Page 100 


\section{Vibration Equipment}

The same vibration testing equipment used in Chapter 3 was used for isolator testing. All testing was done with the $1 \mathrm{U}$ TestPOD due to ease of integration and because there was only a $1 \mathrm{U}$ isolated CubeSat.

\section{Vibration Testing}

\section{Isolator Testing}

Using silicon foam isolators, six tests were run with the Test CubeSat set at three different masses (three sine sweeps and three random vibration tests). All six of these tests were run in the $\mathrm{X}$ axis of the $1 \mathrm{U}$ TestPOD. Figure 73 shows the test setup for the six tests. Note that the accelerometer on the 1U TestPOD malfunctioned, and did not collect data. Figure 74 shows the random vibration response of the CubeSats with masses of $.42 \mathrm{~kg}, .97 \mathrm{~kg}$ and $1.30 \mathrm{~kg}$. Raw data from these tests are in Appendix E. 


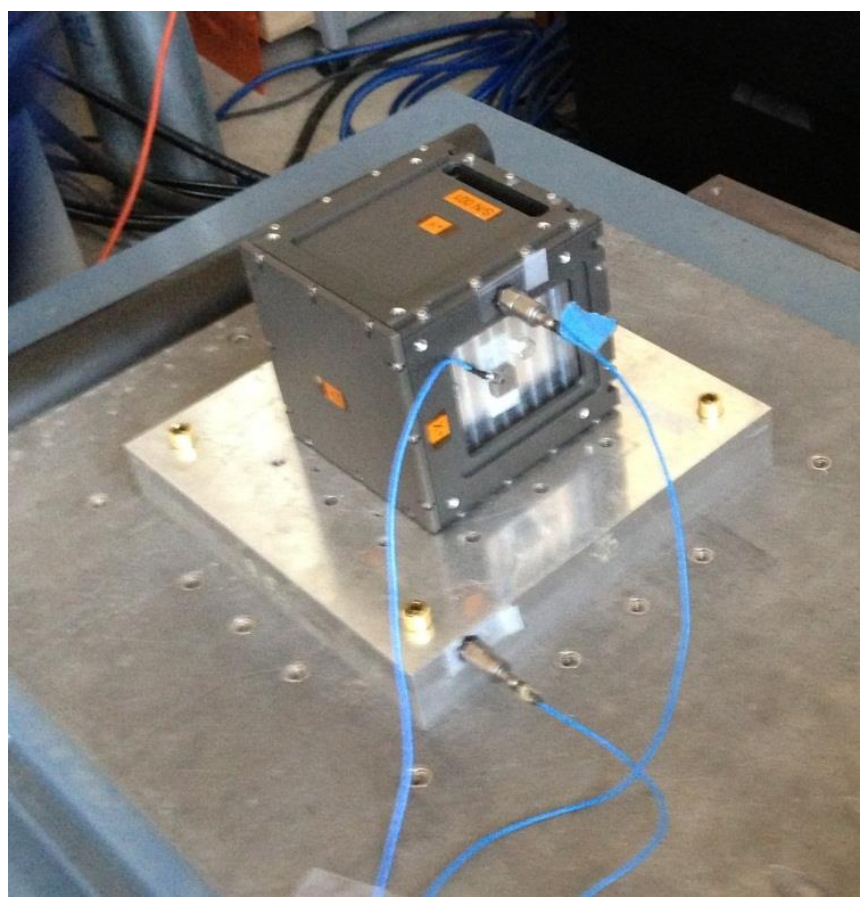

Figure 73: Test setup for three $\mathrm{X}$ axis isolation tests. 


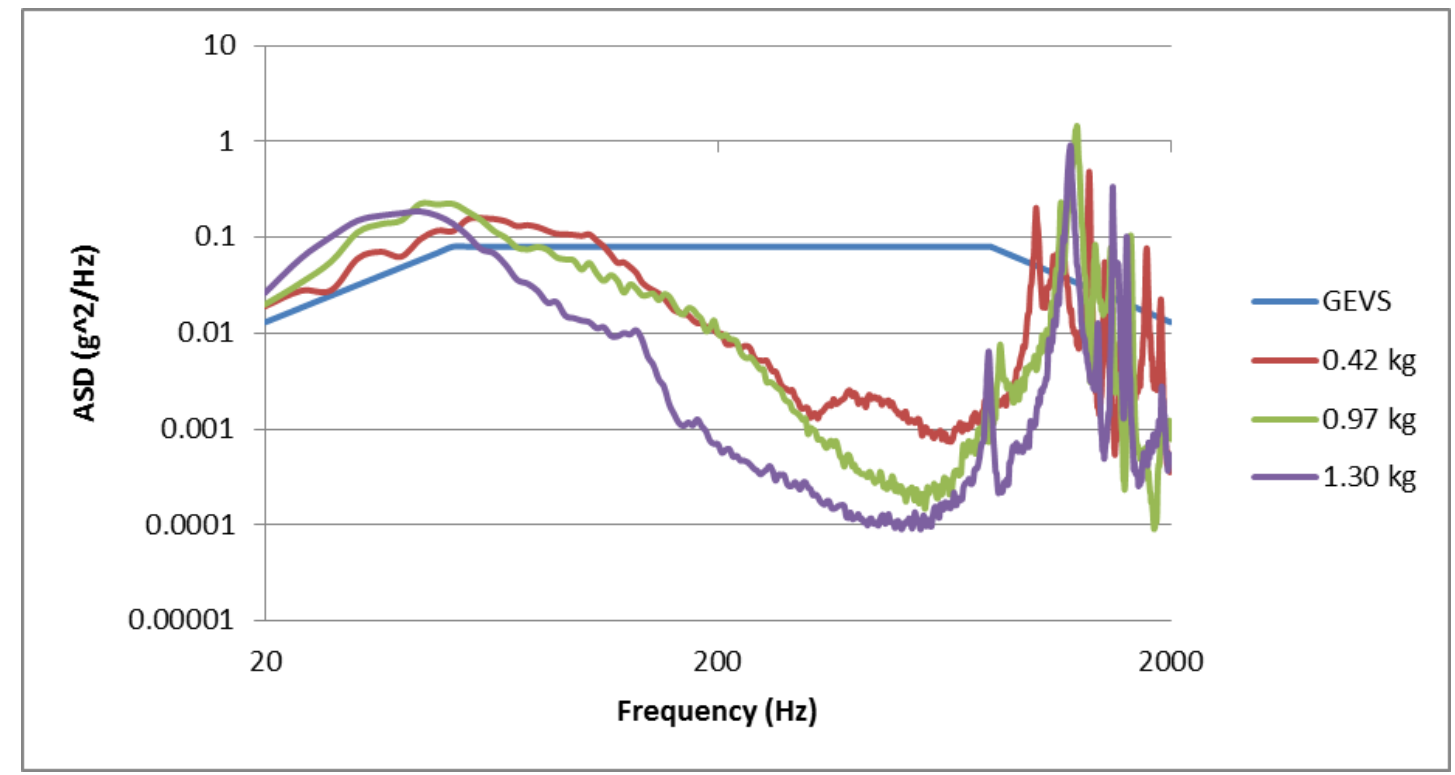

Figure 74: Isolated CubeSat response to random vibration profile.

For comparison, a different material was used for the isolator that was stiffer than the silicon foam, and placed on the $\mathrm{Z}$ axis of the Test CubeSat. The area of the isolator was much smaller, so the overall isolator stiffness was about the same as in the $\mathrm{X}$ axis. Figure 75 shows the test setup for the $\mathrm{Z}$ axis isolator tests, and Figure 76 shows the isolated CubeSat response to a random vibration input. Raw data for these tests is shown in Appendix E. 


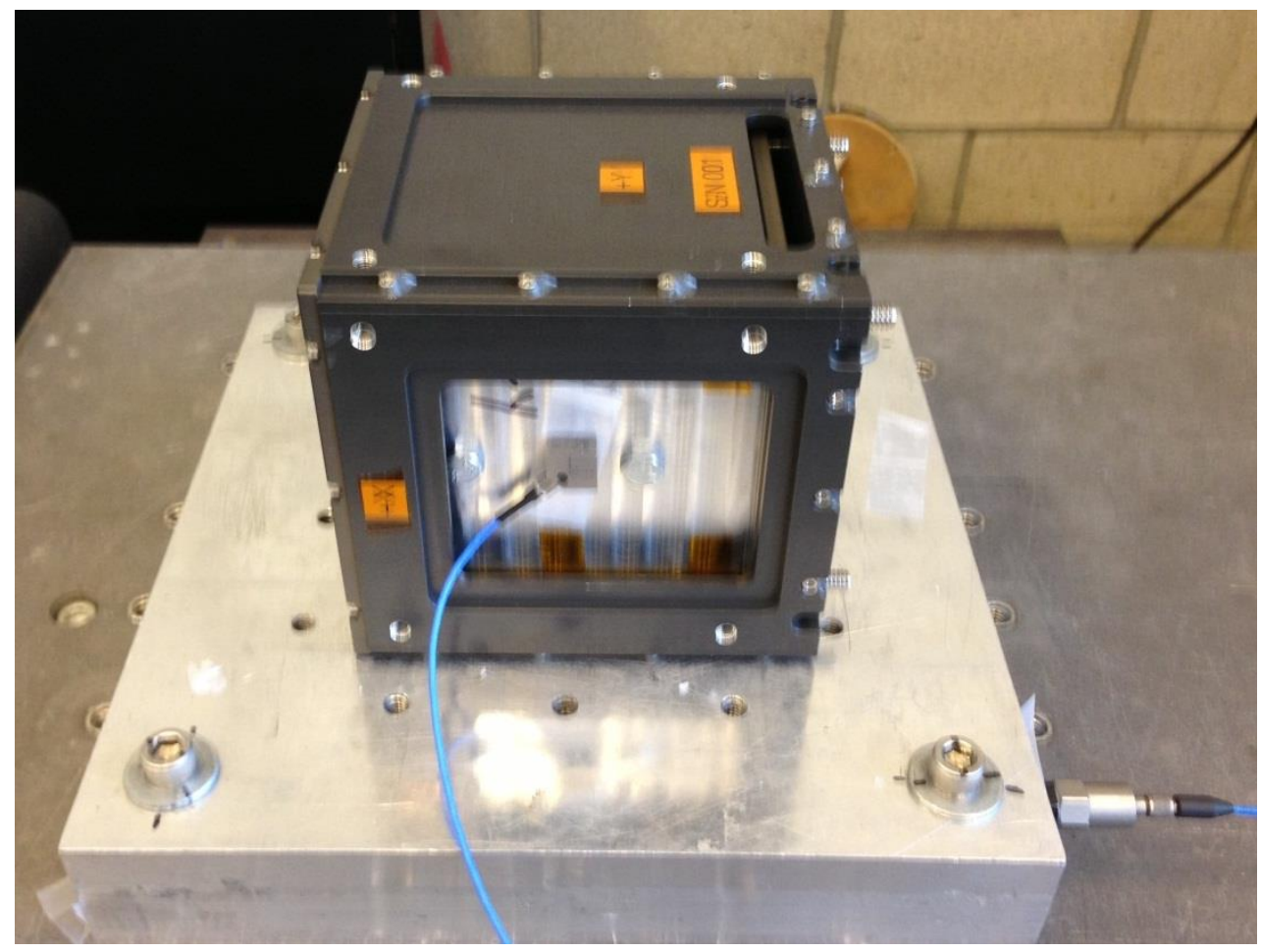

Figure 75: Test setup for $Z$ axis isolator tests.

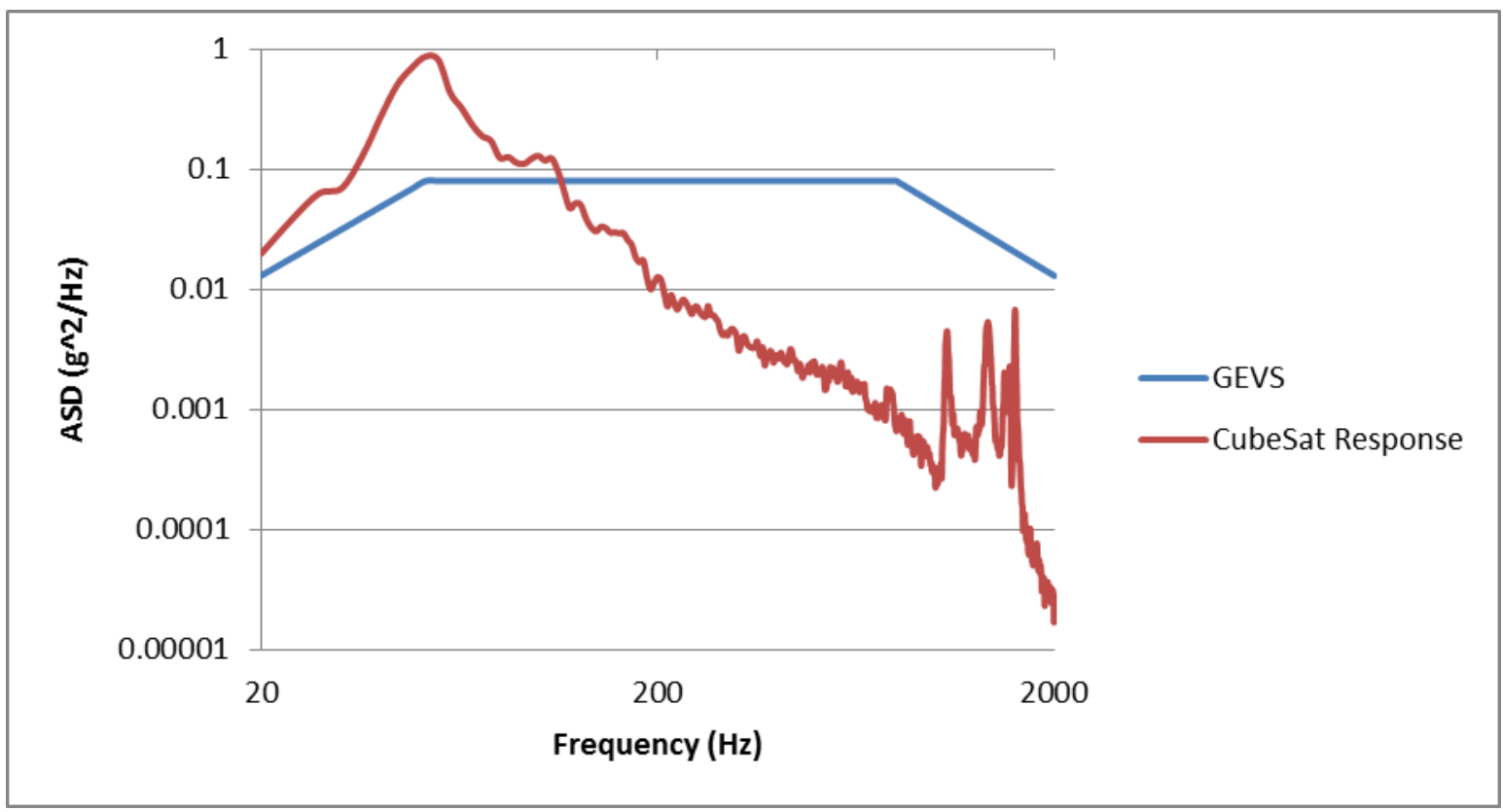

Figure 76: Isolated CubeSat response for comparison case in $\mathrm{Z}$ axis of $1 \mathrm{U}$ TestPOD. 


\section{Analysis of Isolators}

\section{Characterization of Isolator Frequency and Damping Ratio}

A summary of the natural frequencies and damping ratios from the four isolator sine sweep tests are shown in Table 4. The natural frequency was the frequency of maximum transmissibility. The damping ratio was calculated using Equation 7.

Table 4: Natural frequency and damping ratios from isolator sine sweep tests.

\begin{tabular}{|c|c|c|c|}
\hline Case & $\begin{array}{c}\text { Natural } \\
\text { Frequency } \\
(\mathrm{Hz})\end{array}$ & $\begin{array}{c}\text { Amplification } \\
\text { at Resonant } \\
\text { Peak }\end{array}$ & $\begin{array}{c}\text { Damping } \\
\text { Ratio }\end{array}$ \\
\hline $\mathrm{X}$ Isolator-1.3 kg & 52.8 & 2.1 & 0.24 \\
\hline $\mathrm{X}$ Isolator-.97 kg & 76.9 & 1.6 & 0.32 \\
\hline $\mathrm{X}$ Isolator-.47 kg & 105 & 2.1 & 0.24 \\
\hline Z Isolator-1.3 kg & 64.2 & 2.9 & 0.17 \\
\hline
\end{tabular}

Using the mass and isolator frequency data, an isolator frequency versus mass chart was created (refer to Figure 71). Figure 77 shows two plots of isolator frequency versus CubeSat mass. The blue line is from experimental data. The red line is from a linearized stiffness model (assumes stiffness does not change with loads or CubeSat mass). A stiffness value for the isolator was obtain using Equation 2 and the mass and natural frequency data from the $\mathrm{X}$ axis sine sweep of the $.97 \mathrm{~kg}$ Test CubeSat. 
Assuming the same stiffness for the isolators, the isolator frequencies were then calculated for the other Test CubeSat masses using Equation 2.

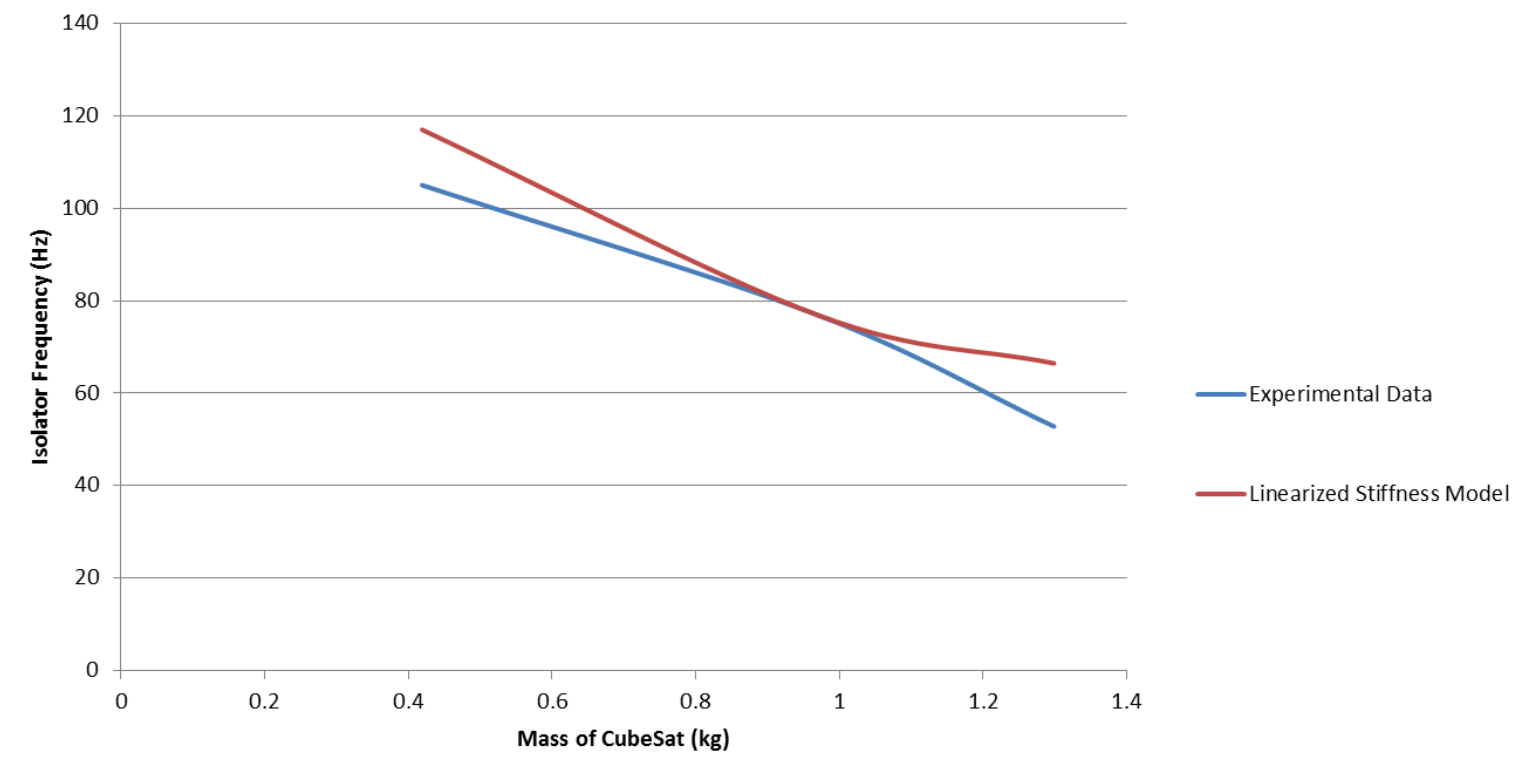

Figure 77: Isolator frequency versus CubeSat mass.

The experimental data shows that the isolator does not follow the trend from linear vibration theory. This is expected, as silicon foam in compression is non-linear. This shows that isolator frequency for this type of system will have to be characterized through thorough testing instead of determined through linear vibration methods.

\section{Comparison to SDOF Model}

\section{SDOF Model from Sine Sweep Data}

Using the sine sweep data from Table 4, a SDOF isolator model was developed to model the isolated Test CubeSat's response to random vibration. Figure 78, Figure 79, 
Figure 80 and Figure 81 show the actual CubeSat response compared to the SDOF isolator approximation.

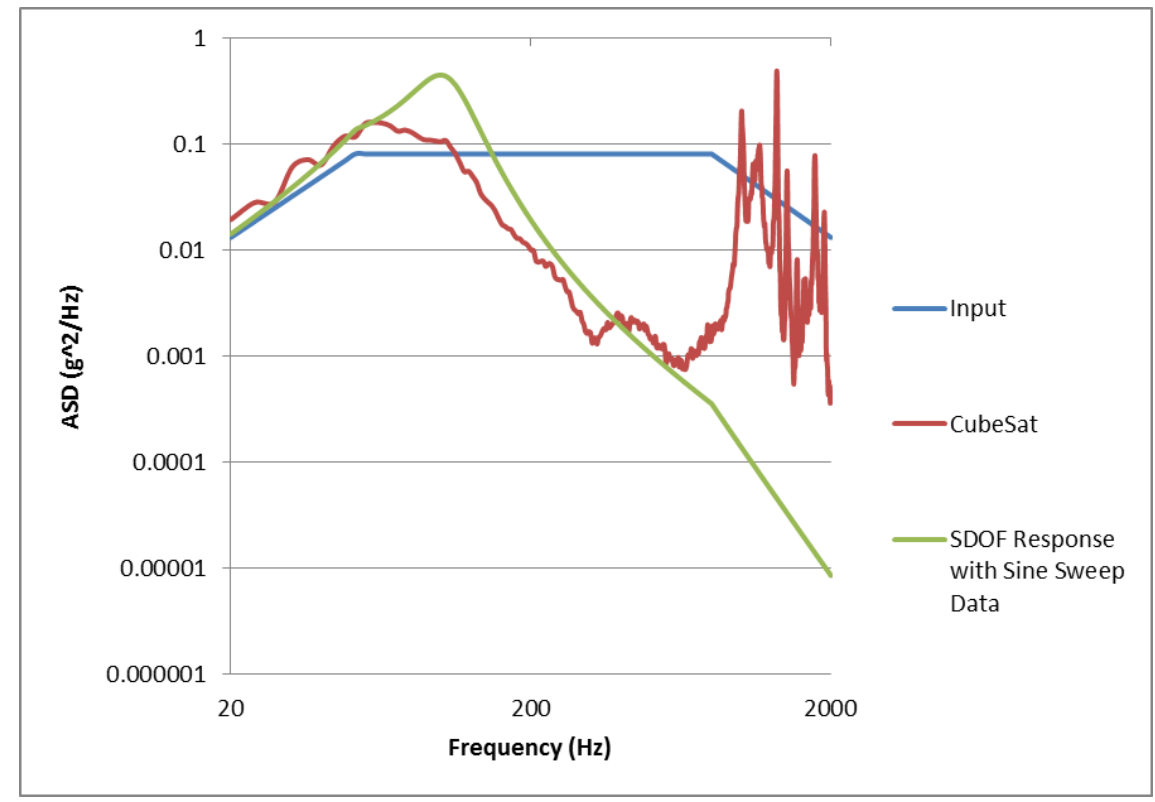

Figure 78: SDOF model of $.42 \mathrm{~kg}$ Test CubeSat using data from Sine Sweep test.

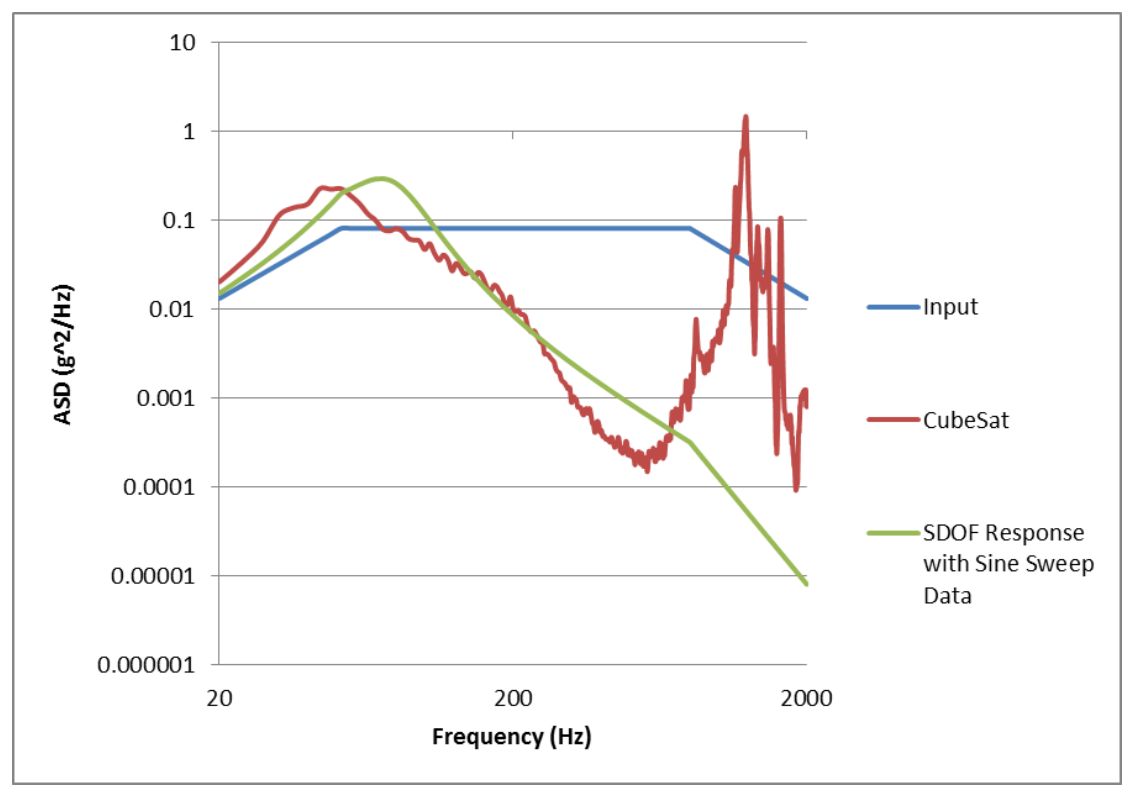

Figure 79: SDOF model of .97 kg Test CubeSat using data from Sine Sweep test. 


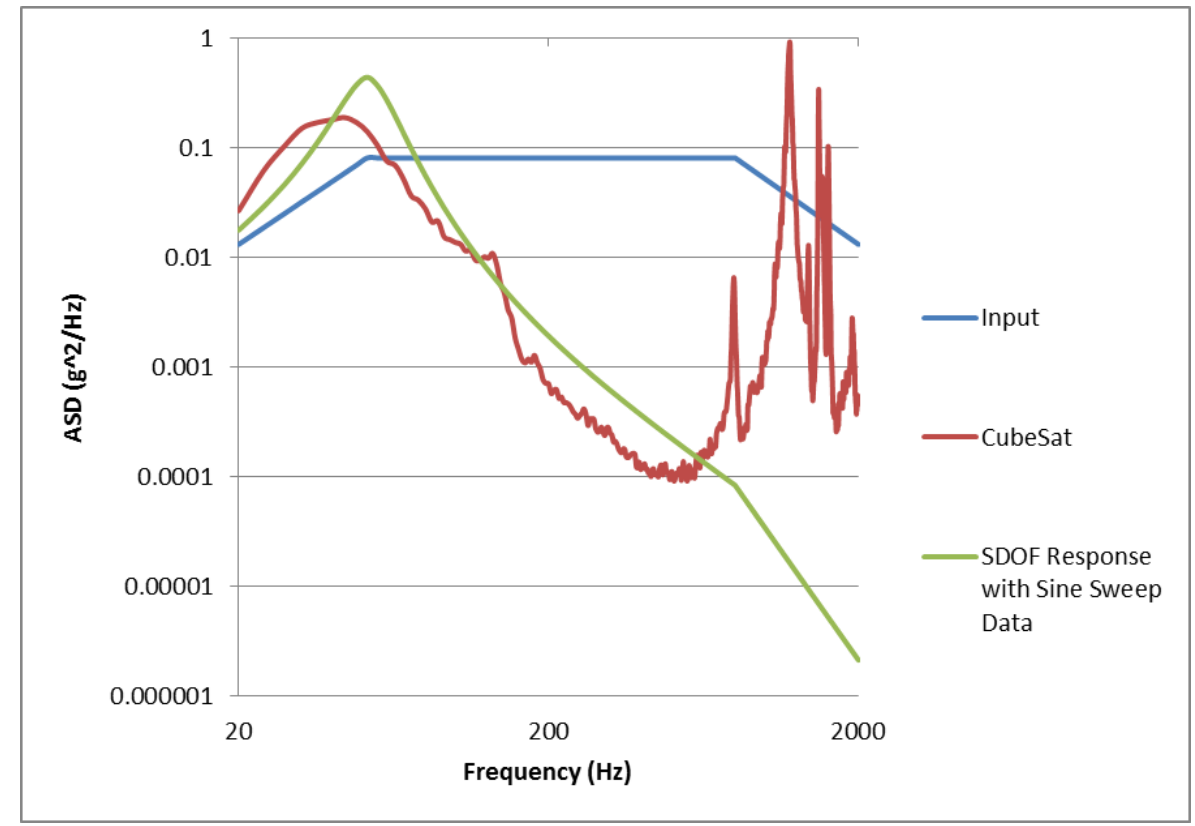

Figure 80: SDOF model of $1.30 \mathrm{~kg}$ Test CubeSat using data from Sine Sweep test.

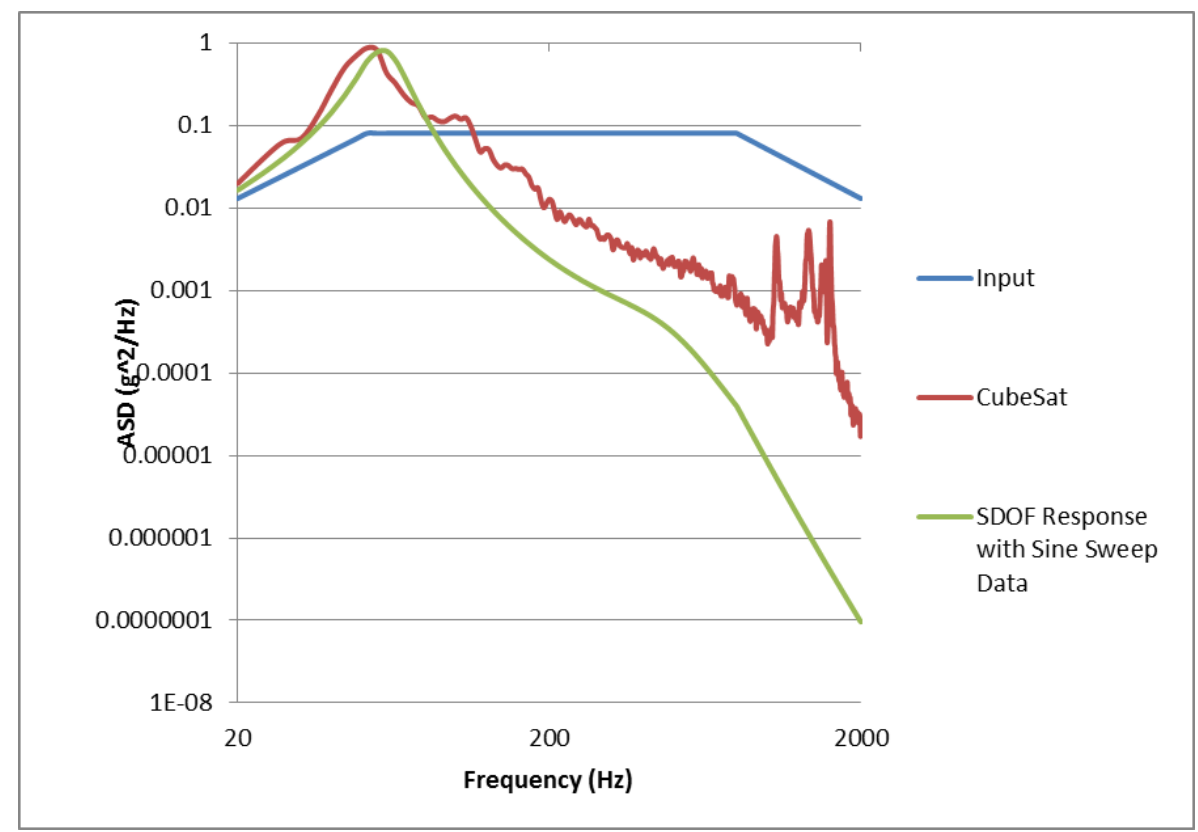

Figure 81: SDOF model of $1.30 \mathrm{~kg}$ Test CubeSat in $\mathrm{Z}$ axis using data from Sine Sweep test.

The natural frequency from the sine sweep data was consistently higher than the natural frequency from the random vibration profile. It is expected that the natural frequency 
would change as the magnitude of vibration input changed, because the isolator material has non-linear stiffnesses. The damping ratio was also much higher for the random vibration profiles than the sine sweeps, except for the $\mathrm{Z}$ axis. This was expected, because in the $\mathrm{X}$ axis, the CubeSat acts like a particle damper, with damping increasing with loads. In the $\mathrm{Z}$ axis, the CubeSat still has no room to rattle, and the damping does not increase with loads.

Note that the peaks in the region of $800-2000 \mathrm{~Hz}$ are from the dynamics of the Test CubeSat, and are not from the deployer or isolation system. This was shown in the FEA of the Test CubeSat (see Appendix C).

\section{Revised SDOF Isolator Model}

Because the sine sweep data did not accurately predict isolated CubeSat loads, revised natural frequency and damping values where applied to the models. These values were chosen to create the best approximation of the actual response with the SDOF model. Figure 82, Figure 83, Figure 84, and Figure 85 show the revised SDOF approximation. 


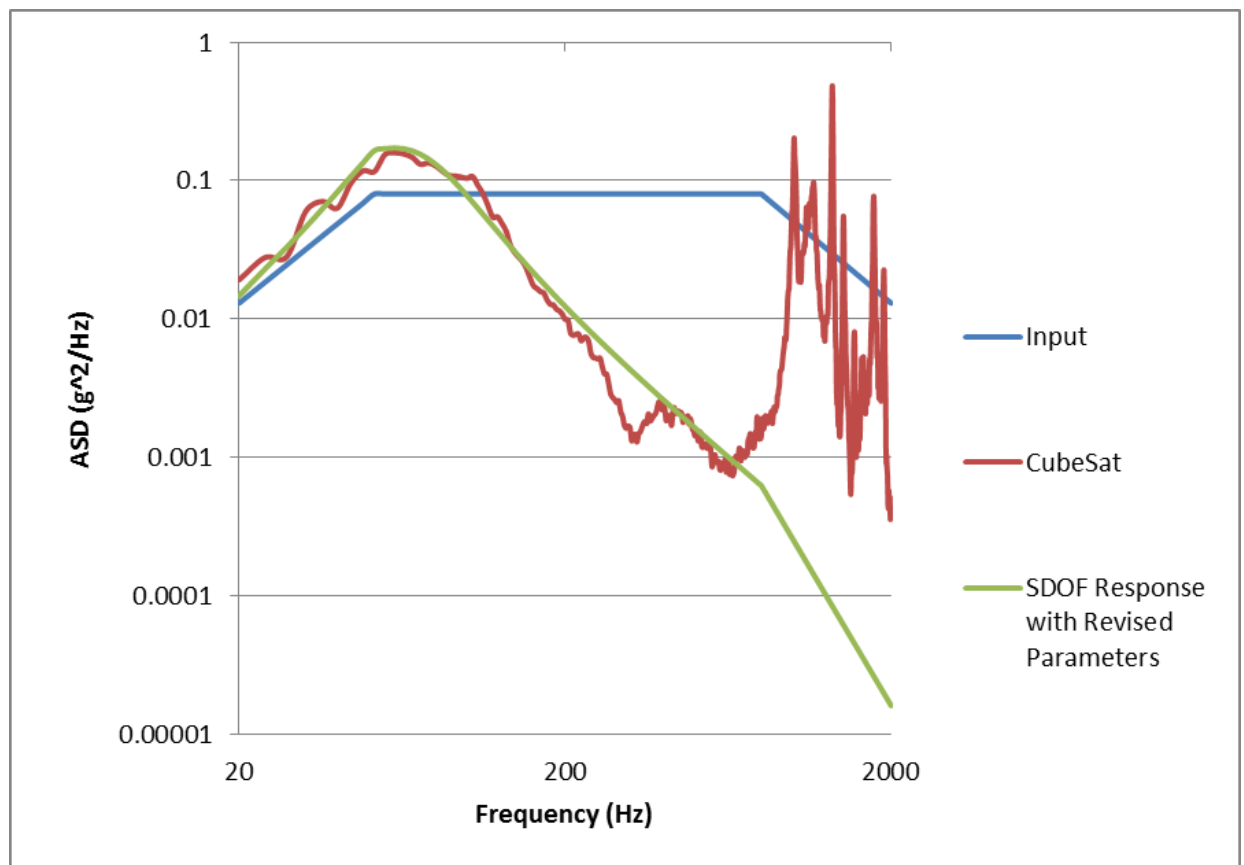

Figure 82: SDOF model of $.42 \mathrm{~kg}$ Test CubeSat in $\mathrm{X}$ axis using revised parameters.

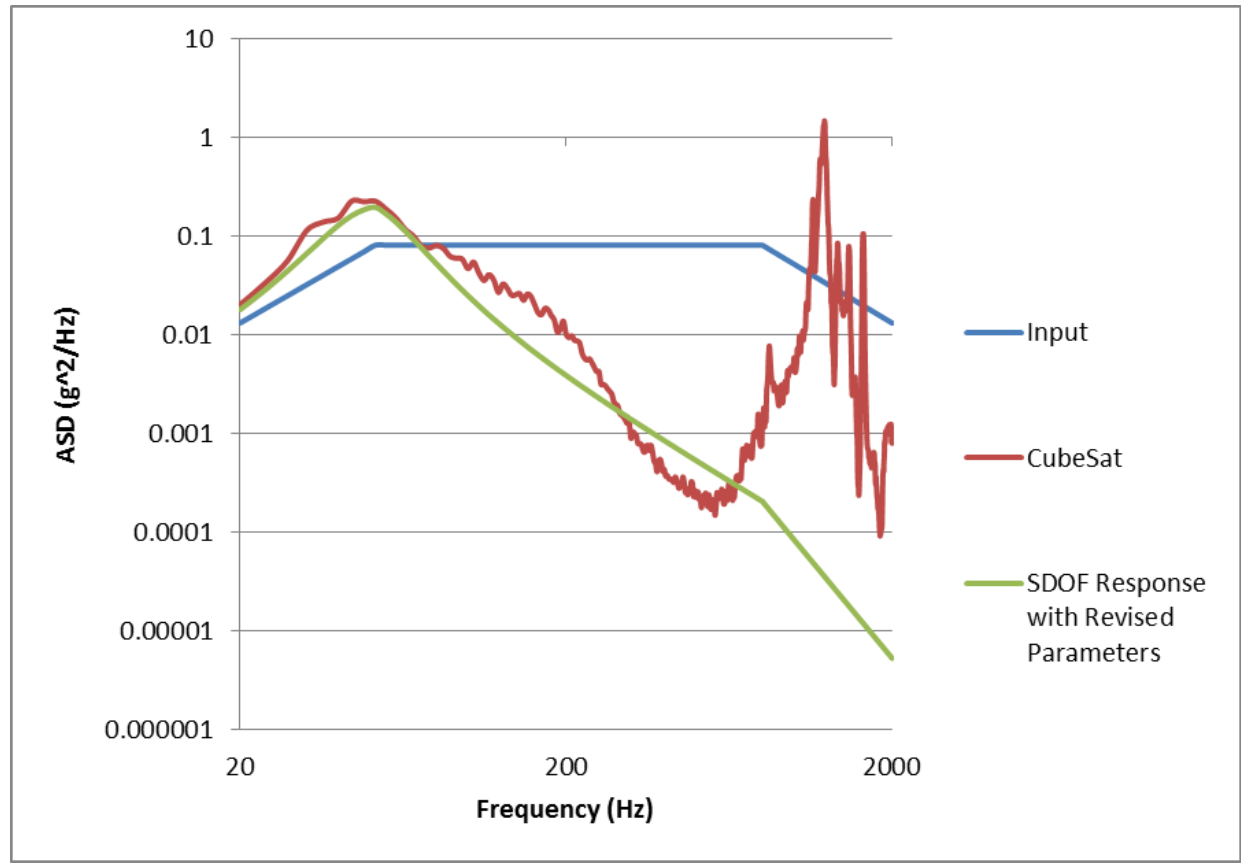

Figure 83: SDOF model of $.97 \mathrm{~kg}$ Test CubeSat in $X$ axis using revised parameters. 


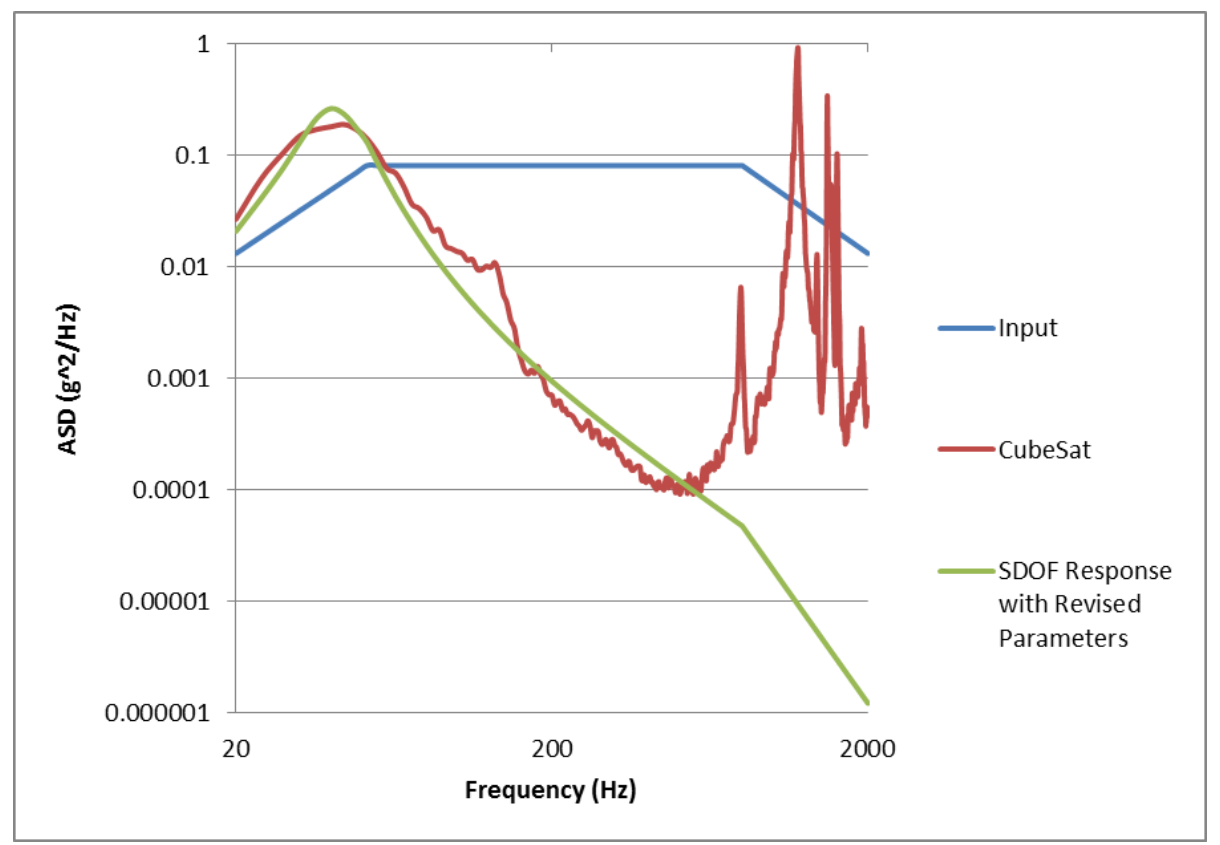

Figure 84: SDOF model of $1.3 \mathrm{~kg}$ Test CubeSat in $\mathrm{X}$ axis using revised parameters.

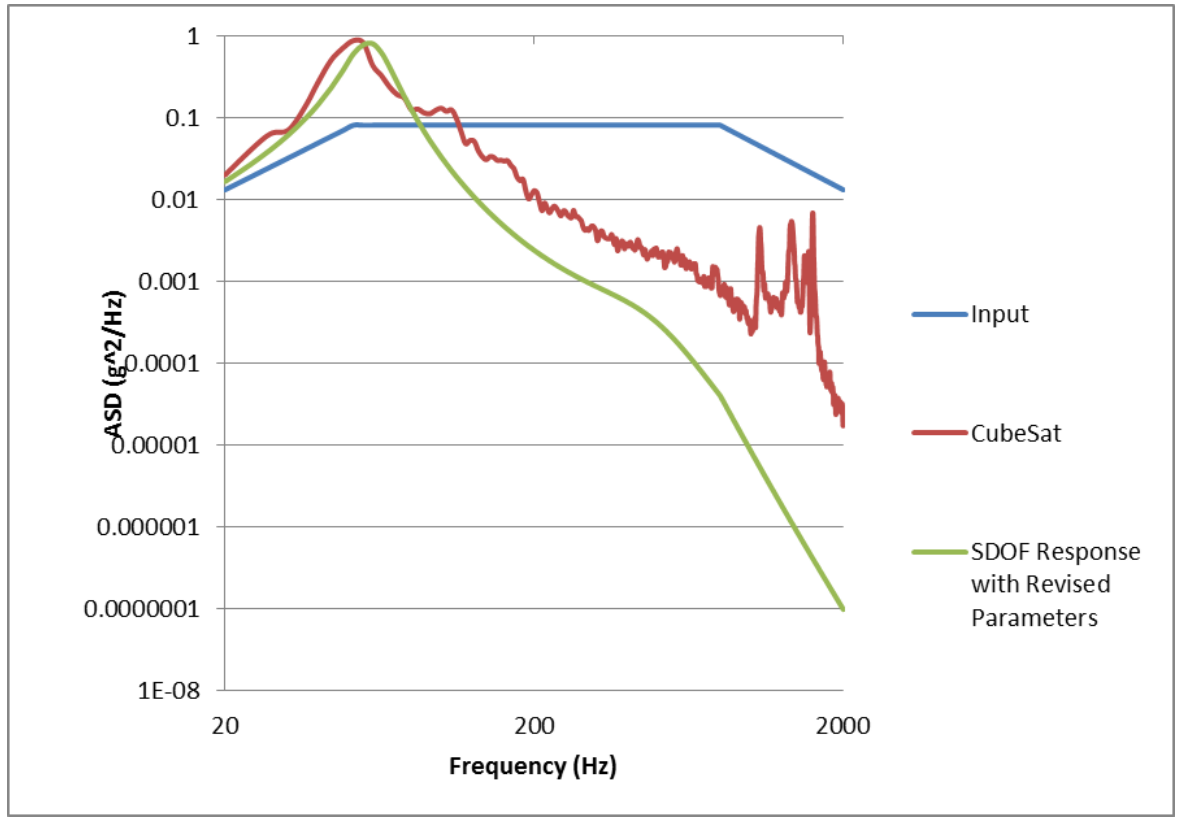

Figure 85: SDOF model of $1.30 \mathrm{~kg}$ Test CubeSat in $\mathrm{Z}$ axis using revised parameters.

Page 111 
The revised model provides a much better approximation. The amplification region is well modeled, as well as the slope of the roll-off in the isolation region.

The $\mathrm{G}_{\mathrm{rms}}$ was calculated from $20-800 \mathrm{~Hz}$ for the actual and theoretical responses of the four random vibration tests run. Data was not taken after $800 \mathrm{~Hz}$ because no attempt was made to characterize and predict the response with the CubeSats's dynamics. Table 5 summarizes the response results and error between actual and theoretical response.

Table 5: Summary of actual versus theoretical isolated CubeSat response from $20-800 \mathrm{~Hz}$.

\begin{tabular}{|c|c|c|c|}
\hline Test & $\begin{array}{c}\text { Actual CubeSat } \\
\text { Response }\left(\mathbf{G}_{\text {rms })}\right.\end{array}$ & $\begin{array}{c}\text { Isolator Model } \\
\left(\mathbf{G}_{\mathbf{r m s}}\right)\end{array}$ & $\begin{array}{c}\text { Percent Error } \\
(\boldsymbol{\%})\end{array}$ \\
\hline X Axis-.42 kg & 3.63 & 3.69 & 1.65 \\
\hline X Axis-.97 kg & 3.63 & 3.69 & 1.65 \\
\hline X Axis-1.3 kg & 3.48 & 2.91 & 16.4 \\
\hline Z Axis-1.3 kg & 5.52 & 4.50 & 18.5 \\
\hline
\end{tabular}

What this illustrates is that a linear SDOF isolator model can be used to approximate loads in a deployer with an isolation system. To obtain the correct damping and natural frequency values, thorough characterization would have to be performed at the correct vibration levels.

\section{Isolator Effectiveness}

The data from random vibration testing shows the CubeSat is effectively isolated from deployer dynamics and vibration loads are effectively mitigated. Figure 86 shows 
the $\mathrm{X}$ axis response of the Test CubeSats set at three different masses along with a fixed CubeSat response (test number 8-1 from Chapter 3).

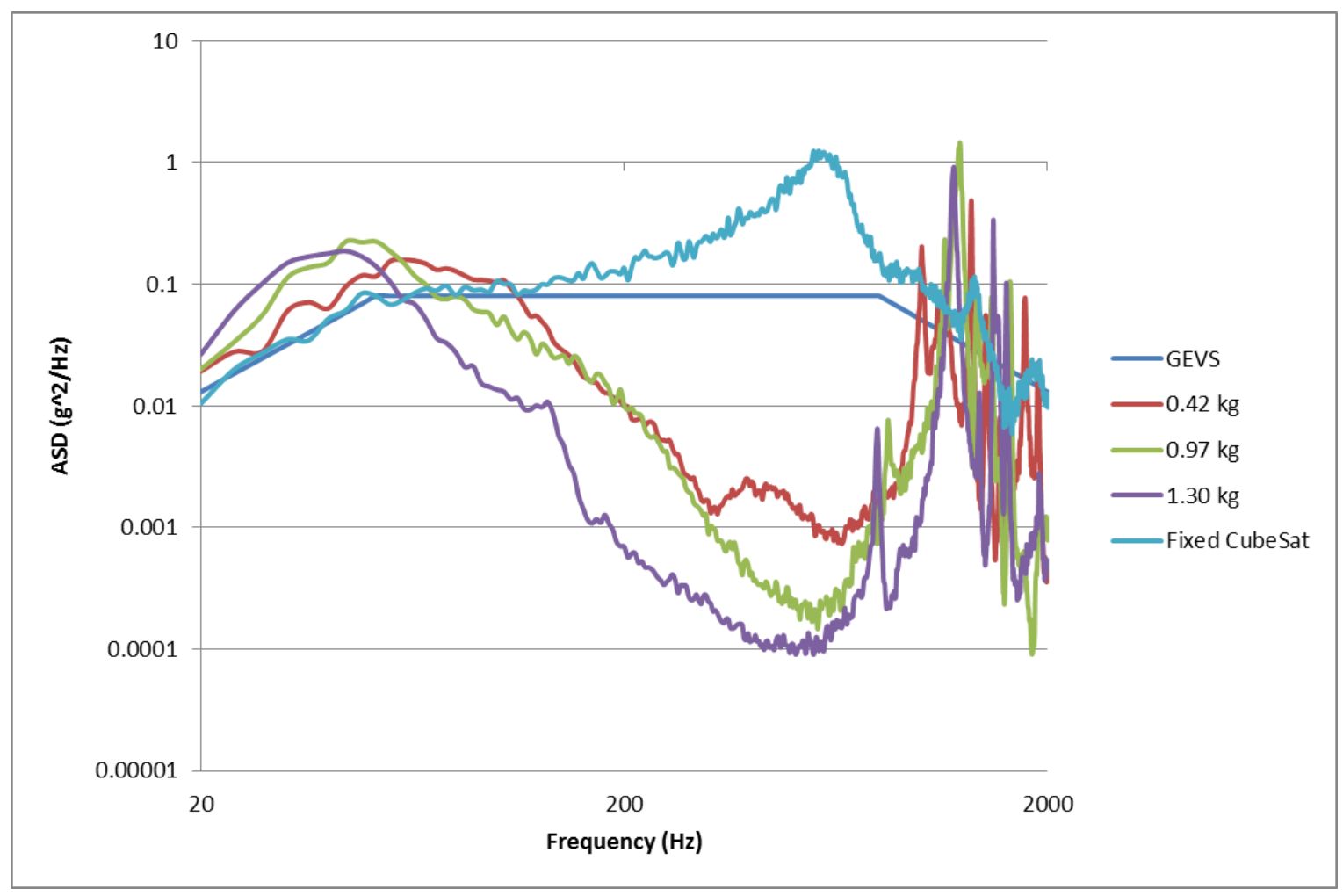

Figure 86: Isolated CubeSat response with fixed CubeSat response.

Notice how the fixed CubeSat resonates with the deployer, but the isolated CubeSats do not. This is very similar to the unfixed case for CubeSats in the $\mathrm{X}$ and $\mathrm{Y}$ axes of the PPOD.

\section{Conclusions}

The Test CubeSat provides an accurate representation of how CubeSats will be constrained in the proposed deployer in Chapter 5. The material properties, stiffness and thickness are very similar to what would be implemented in a flight model. Even though 
the isolators were placed on the CubeSat, the dynamics would still be very similar to isolators placed on the deployer.

Data from testing of this model showed that:

- The isolated constraint can be modeled with a linear SDOF transfer function

- Need thorough characterization for all possible CubeSat masses and loads to obtain natural frequency and damping data

○ Good isolator design and characterization can yield a system that can be approximated to within $10 \%$

- Isolators mitigate vibration environment

○ Data shows CubeSat is isolated from deployer dynamics, and deployer amplification is avoided

The isolators provide a method to reduce CubeSat vibration loads while providing a constraint that lends itself to vibration analysis. It is clear from the data that the isolated CubeSat does not respond with deployer dynamics. Analysis of the isolated response shows that, if good natural frequency and damping values are chosen, the response could be predicted to within $2 \%$. While some cases were off by almost $19 \%$, these tests prove the feasibility that this constraint can provide a consistent and analyzable constraint.

Dialing the spring plungers out and allowing the CubeSats to displace in all axes without isolators would technically reduce the overall vibration while constraining the CubeSat in a predictable manner. However, this author recommends at least a small 
amount of isolator material to reduce impulse time of impacts and lower possible shocks that are not measured with the current accelerometers. The thicker the isolator, the more linear it will act and the easier the system will be to analyze. 
CHAPTER 7: Conclusion

\section{CubeSat-Deployer Model}

- A model was created for the CubeSat constraint inside the deployer

- CubeSat is fixed in the $\mathrm{Z}$ axis

- Can be modeled with linear vibration methods

- CubeSat can be modeled as fixed or isolated from deployer in X and Y axes

- Based on many factors that can change during test

- Difficult to determine what model to use

- If isolated, a SDOF isolator transfer function must be added between CubeSat and deployer

- Deployer's dynamics affect CubeSat loads when CubeSat is fixed

- If CubeSat is isolated, deployer dynamics have much less of an effect

- Deployers that fix the CubeSat increase loads compared to deployers that have unconstrained CubeSats

○ Fixed CubeSats see "deployer amplification"

- Fixed CubeSat in very stiff deployer is worst case

○ Isolated CubeSats avoid deployer amplification

○ Loads only considered from $20-2000 \mathrm{~Hz}$

There were several models created for the CubeSat-deployer system. The spring plungers in the P-POD successfully fix the CubeSat in the $\mathrm{Z}$ axis, allowing the CubeSat- 
deployer system to be modeled using linear vibration methods in this axis. Two models were needed for the $\mathrm{X}$ and $\mathrm{Y}$ axes. When friction from the spring plungers is high enough to fix the CubeSat in the P-POD, CubeSats can be modeled just like the $\mathrm{Z}$ axis. When the friction force is not high enough, the CubeSats can translate in the $\mathrm{X}$ and $\mathrm{Y}$ axes and are isolated from the deployer. In this case, a SDOF isolator transfer function must be added between the CubeSat and deployer.

While these models can approximate CubeSat loads, it is very difficult to determine what model to use in the $\mathrm{X}$ and $\mathrm{Y}$ axes. The friction force from the spring plungers is based on many factors that can only be obtained empirically, making it difficult to know if the CubeSat is fixed or isolated from the deployer. Furthermore, it has been shown that the CubeSats can start fixed, and then settle into an isolated position in the middle of the test. This inconsistent constraint makes analysis of CubeSat loads difficult in the $\mathrm{X}$ and $\mathrm{Y}$ axes. The $\mathrm{Z}$ axis constraint is consistent for all CubeSats and deployers.

When the CubeSat is fixed in a deployer, the deployer's dynamics greatly affect the CubeSat response. CubeSats resonate with the deployer's resonance, changing the response of CubeSats in different deployers. Because the CubeSat responds with the deployer's resonant peaks, deployers can magnify loads for fixed CubeSats. This deployer amplification can raise loads to over twice the input of a random vibration profile. The worst case is for very stiff deployers with fixed CubeSats, such as the $\mathrm{Z}$ axis of the $1 \mathrm{U}$ TestPOD. 
If the CubeSat is allowed to translate in the deployer (like in the $\mathrm{X}$ and $\mathrm{Y}$ axes), the deployer's dynamics have much less of an effect. Because the CubeSat does not resonate with the deployer, there is no deployer amplification. Vibration data shows that, between $20-2000 \mathrm{~Hz}$, vibration loads are much lower for unconstrained CubeSats than fixed CubeSats due to this isolation effect.

\section{Proposed Design Concept for Future Deployer}

- A new deployer is needed for the continual progress of the CubeSat program

- A deployer is needed that constrains CubeSats in a predictable and analyzable manner

- A deployer is needed that mitigates vibration loads

- This future deployer should constrain the CubeSat so it is isolated from the deployer

- Avoids deployer amplification caused by fixing the CubeSat

- Isolators should be placed in between the deployer and CubeSat to provide a predictable constraint that reduces vibration loads

- Isolators provide an isolator frequency that can be characterized and set to mission specific values

○ Isolators mitigate metal on metal impacts from the deployer and CubeSat

- Isolator can be modeled with linear SDOF transfer function 
Analysis of CubeSats in the P-POD shows that vibration loads are inconsistent and unpredictable due to the current canisterized constraint. A new deployer must be developed that constrains the CubeSat in a more predictable manner. This deployer should also mitigate input vibration instead of amplifying it.

A concept for a future deployer was developed that isolates CubeSat from the deployer's dynamics. Isolation prevents deployer amplification while providing a consistent constraint for the CubeSat. Isolator material, such as a silicon rubber or foam, can be sandwich in the deployer rails, pusher plate and door. This allows for specific isolator frequency to be chosen for the CubeSats based on the mission specific random vibration profile. Because the CubeSats are isolated in all axes, there is no inconsistency in the constraint in the $\mathrm{X}$ and $\mathrm{Y}$ axes, as there is with the current P-POD.

Testing has shown the isolators constrain the CubeSat in a manner that vibration analysis can be performed using linear SDOF transfer functions. Characterization of an isolator frequency and damping ratio is all that is needed to predict loads in all three axes. If the CubeSat were fixed, an entire MDOF transfer function would need to be developed using FEA or thorough experimental characterization. It would be much easier to characterize the SDOF isolator system than an MDOF deployer.

Testing has also shown that isolation effectively mitigates input vibration. The isolators successfully isolate the CubeSats from the deployer's peaks. This leads to loads that are up to four times less than fixed CubeSats. The results from the isolators are very similar to the results from the unconstrained CubeSats in the $\mathrm{X}$ and $\mathrm{Y}$ axes in the current P-POD. This shows that, as long as the CubeSats have some room to displace, deployer amplification can be avoided. If the CubeSats impacting the rails are 
not a concern, loads can be reduced by simply allowing the CubeSat to translate in all axes.

\section{Final Thought}

Canisterized constraints using rails appear to be a good way to constrain a payload from a vibration standpoint as long as nothing is fixing the payload in any axis (as the spring plunger do in the current P-POD). Because the payload can translate in all axes, it is isolated from high frequency loads. Because the rail to rail impacts act like a particle damper, the damping is very high and the low amplitude magnification associated with isolators is small. Shock created from rail to rail impacts can be mitigated with a soft material sandwiched in the rail with minimal thickness. The result is an isolation system with more damping and less displacement than standard outsidethe-box isolators that can be utilized for CubeSat deployers, CubeSats, and even avionics boxes and other payloads on launch vehicles and spacecraft. 


\section{REFERENCES}

Bashbush, Veronica. Characterization of the Internal and External Environments of the CubeSat P-POD and Test Pod. Thesis. California Polytechnic State University, San Luis Obispo, 2004.

Brummitt, Marissa. Development of CubeSat Vibration Testing Capabilities for the Naval Postgraduate School and Cal Poly San Luis Obispo. Thesis. California Polytechnic State University, San Luis Obispo, 2010.

Irvine, Tom. Avionics Isolation Design Guidelines. Tech. N.p.: Vibration Data, 2009.

Jedrich, Nicholas M., and Scott C. Pendleton. Isolation Systems for Electronic Black Box Transportation to Orbit. Tech. Palo Alto, CA: CSA Engineering.

Marekich, (2012), Passive Vibration Isolation Drawing [ONLINE]. Available at:http://en.wikipedia.org/wiki/File:Passvib1.svg 


\section{APPENDICES}
A. CubeSat Standard
B. Integrated CubeSat Examples
C. FEA on mass model and Test CubeSat
D. Vibration Test Setup and Data from Chapter 3
E. Vibration Test Setup and Data from Chapter 6 
APPENDIX A: CubeSat Design Standard

Page 123 


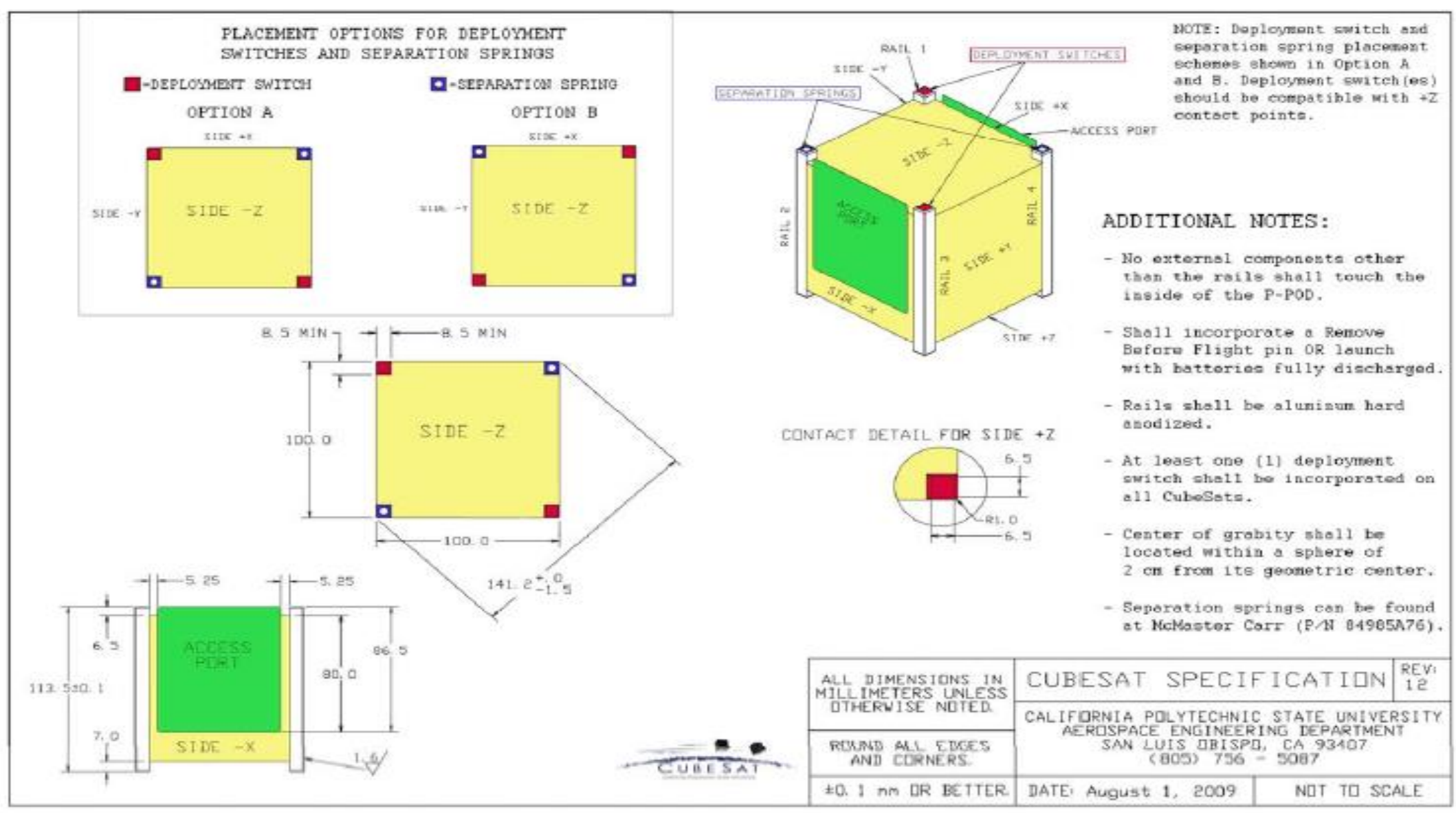

Figure 87: 1U CubeSat Design Standard. 


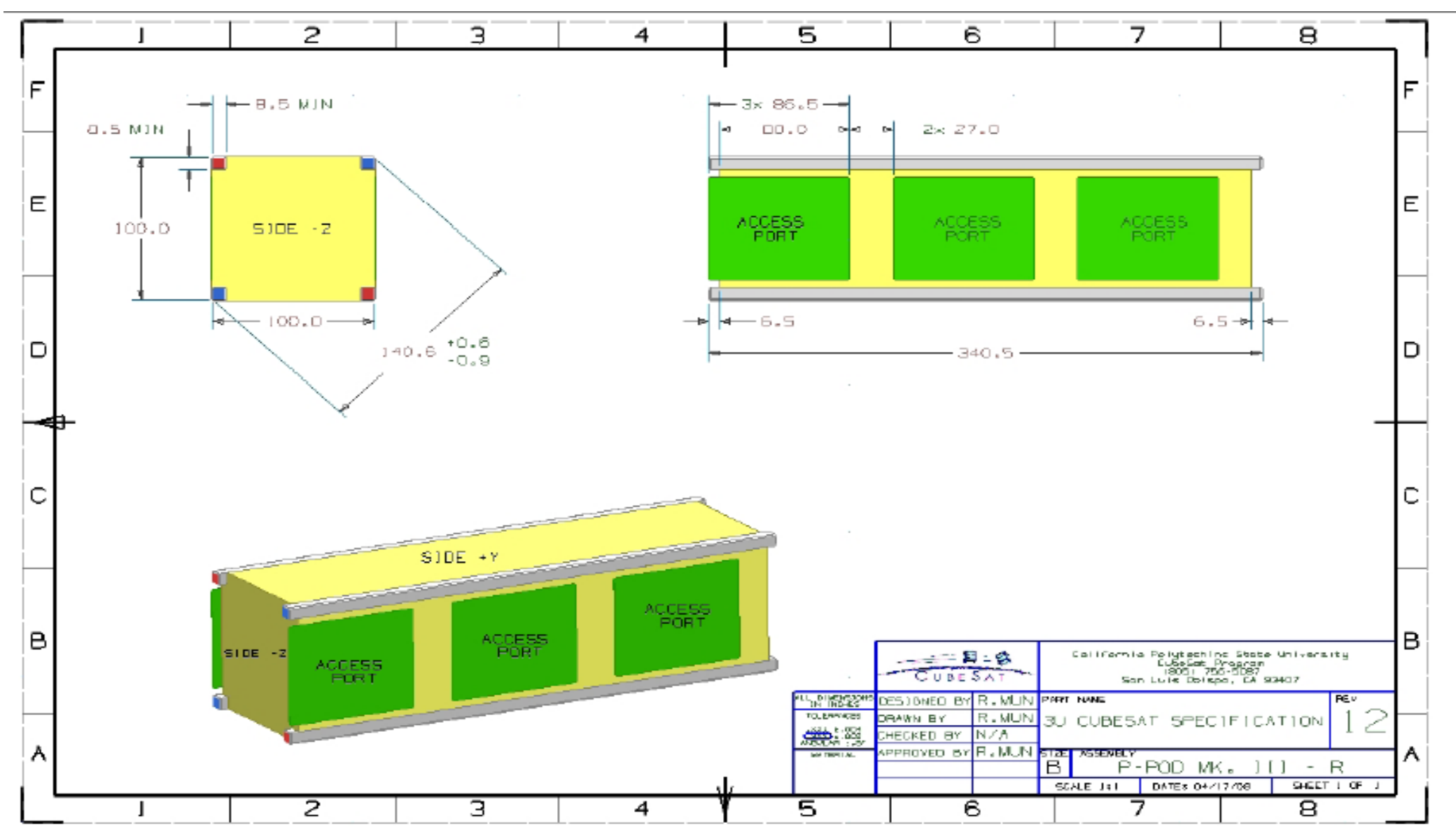

Figure 88: 3U CubeSat Design Standard. 
APPENDIX B: Sample Integrated P-PODs

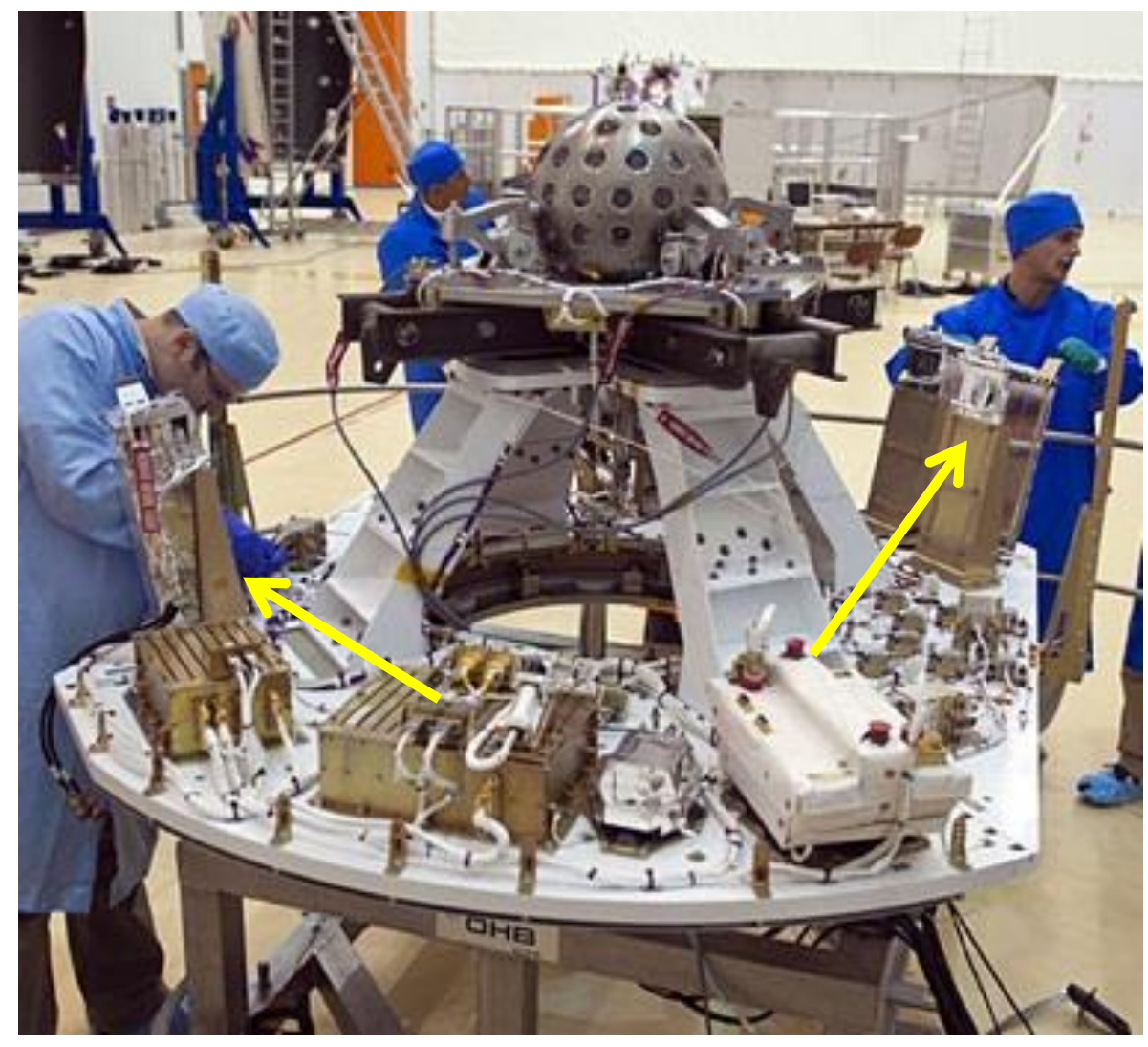

Figure 89: 3 P-PODs integrated on Vega Launch Vehicle.

Page 126 


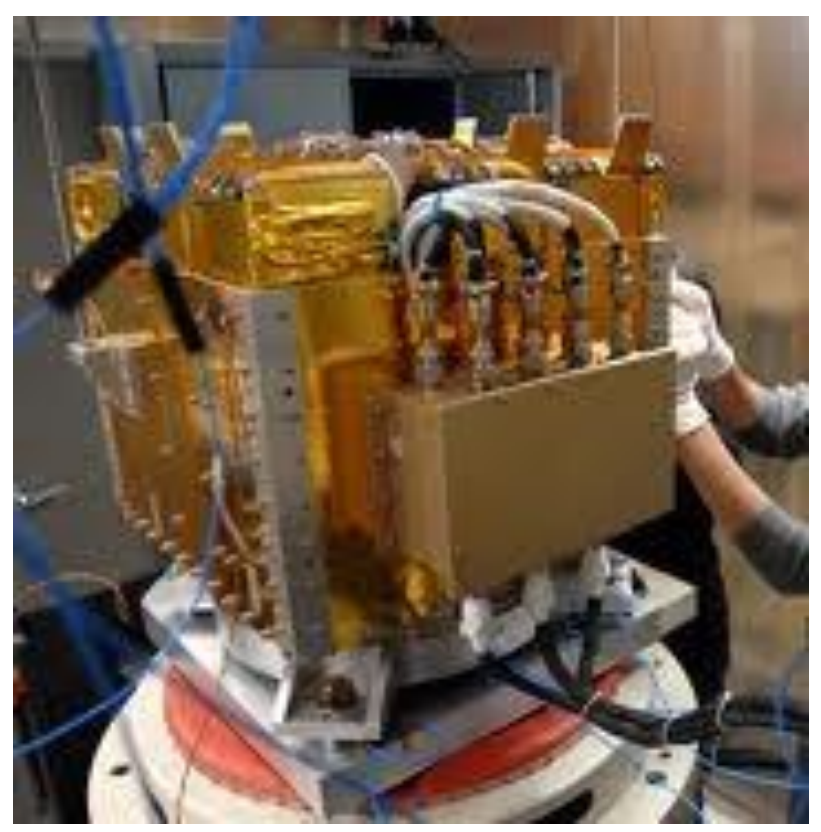

Figure 90: 8 P-PODs on NPS CuL.

Page 127 


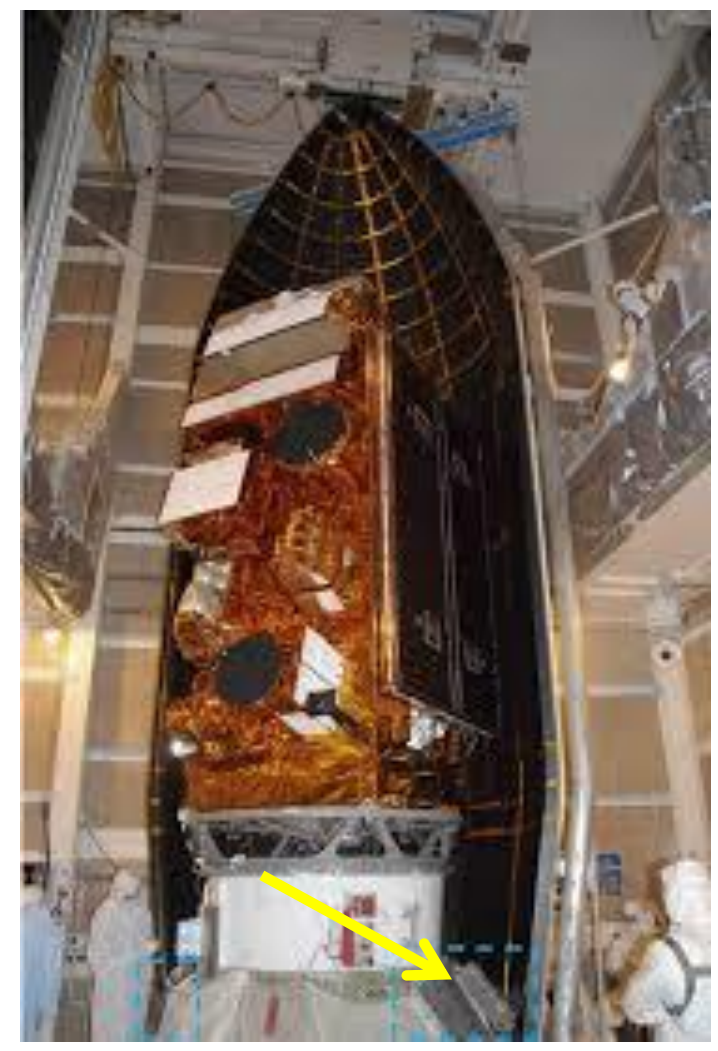

Figure 91: 3 P-PODs integrated on Delta II.

Page 128 
APPENDIX C: FEA from CubeSat Mass Model and Test CubeSat

\section{CubeSat Mass Model}

A normal modes solution (solution 103) was run in NX/NASTRAN on a finite element model (FEM) of the CubeSat mass model to obtain the frequency of the first resonant peak. If the peak is over $2,000 \mathrm{~Hz}$, the mass model can be modeled as rigid for the analysis in this thesis. The FEM was created out of 3D Hex meshes with the material properties of aluminum 6061-T6 (elastic modulus of $10.1 \mathrm{e} 6 \mathrm{psi}$, density of 0.1 pounds per cubic inch and a Poisson's ratio of 0.3). There were no constraints on the FEM to obtain a worst case first mode. Figure 92 shows the first mode of the CubeSat mass model (that is not a rigid-body mode) at $2,160 \mathrm{~Hz}$. The mesh is included in the figure.

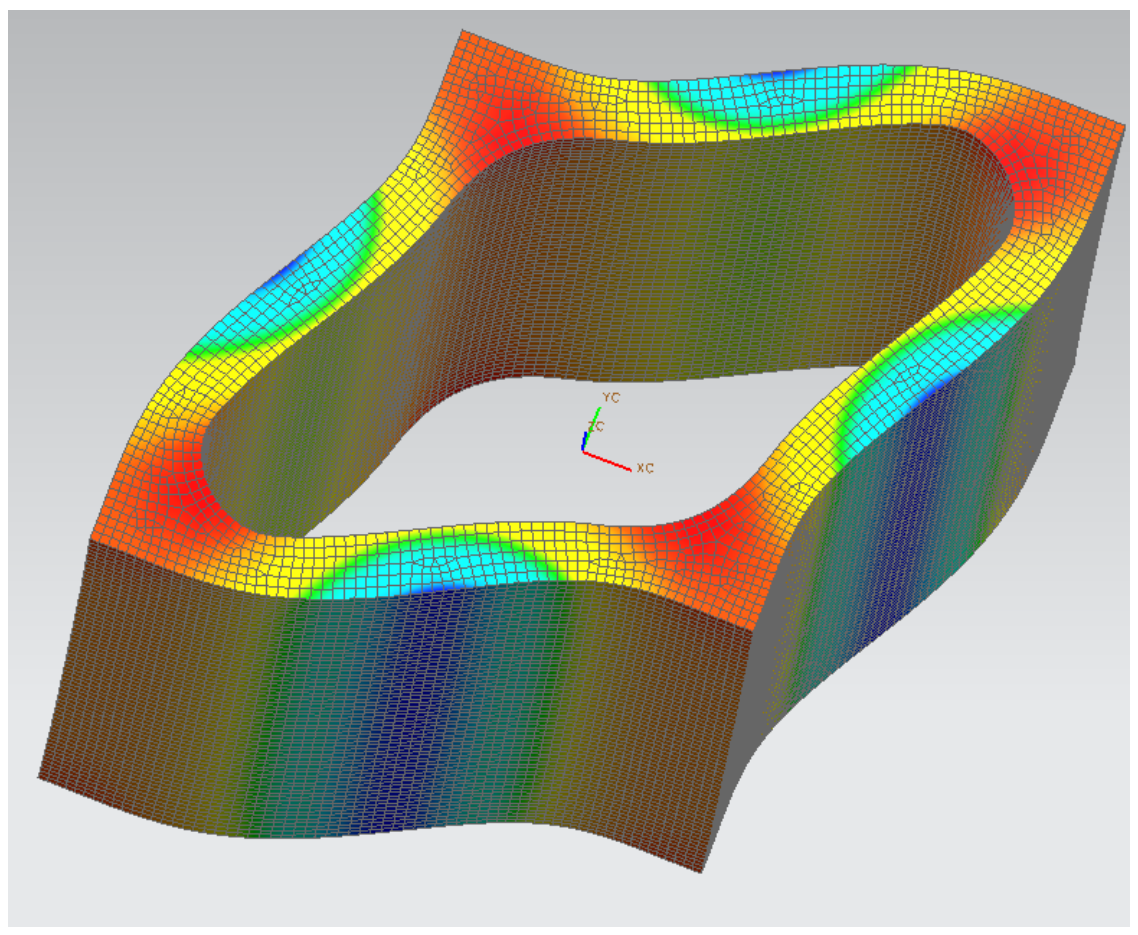

Figure 92: First mode of CubeSat mass model $(2160 \mathrm{~Hz})$.

Page 129 
A convergence study was performed to ensure enough degrees of freedom were used to in the FEM of the CubeSat mass model. Figure 93 shows that, at 124,984 degrees of freedom, the eigenvalues sufficiently converged.

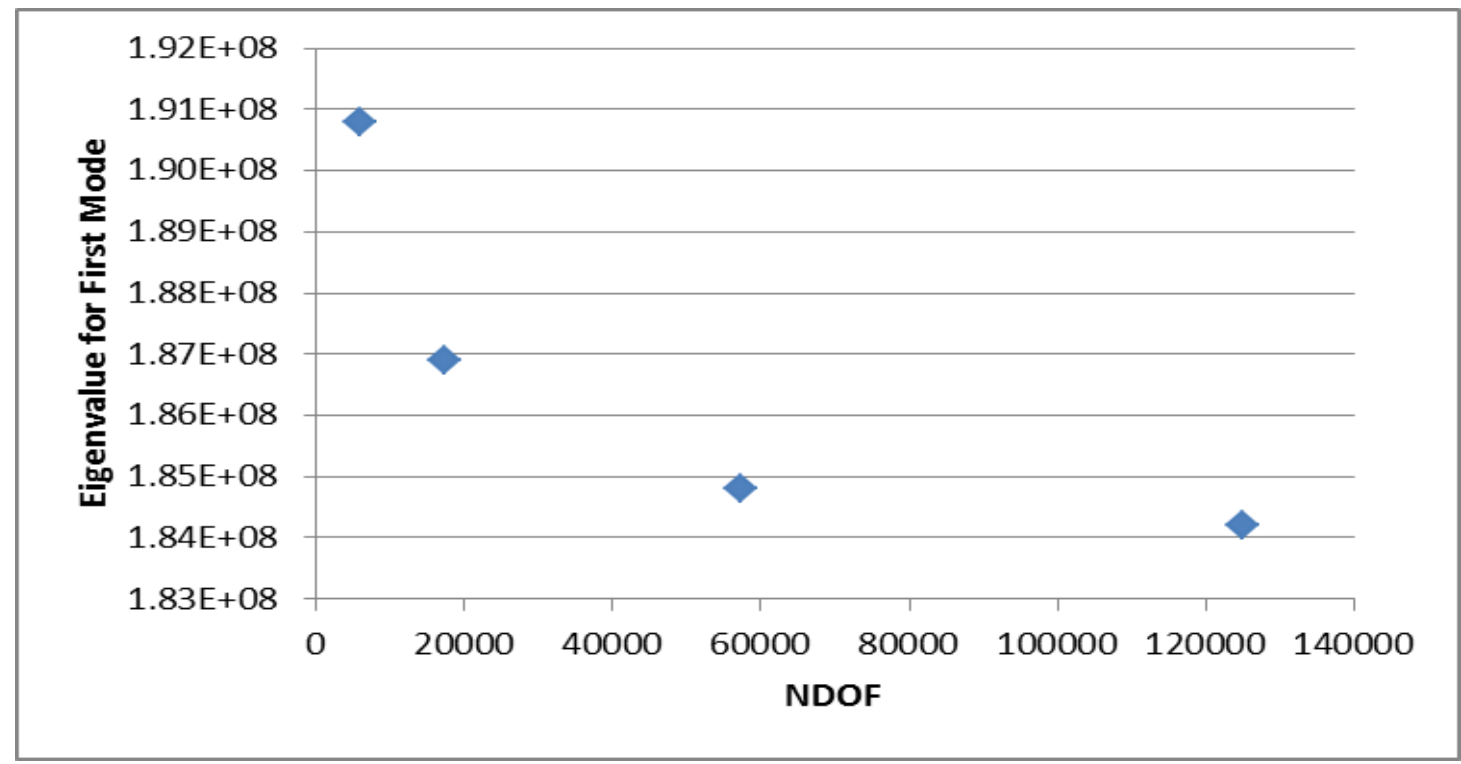

Figure 93: Convergence study for CubeSat mass model.

This FEA study shows that the CubeSat mass model can be modeled as rigid inside the deployer from 20-2000 Hz.

\section{Test CubeSat}

A modal study was performed on the Test CubeSat to ensure the peaks seen from testing in the frequency range of $800-2000 \mathrm{~Hz}$ were from the Test CubeSat's resonant frequencies. A normal modes solution (solution 103) was run on a FEM of the Test CubeSat in NX/NASTRAN. The FEM was created out of 2-D shell elements using the same material properties as the CubeSat mass model (aluminum 6061-T6). Figure Page 130 
94, Figure 95, and Figure 96 show modes that would produce peaks in the $800-2000 \mathrm{~Hz}$ range. Meshes are included in the figures.

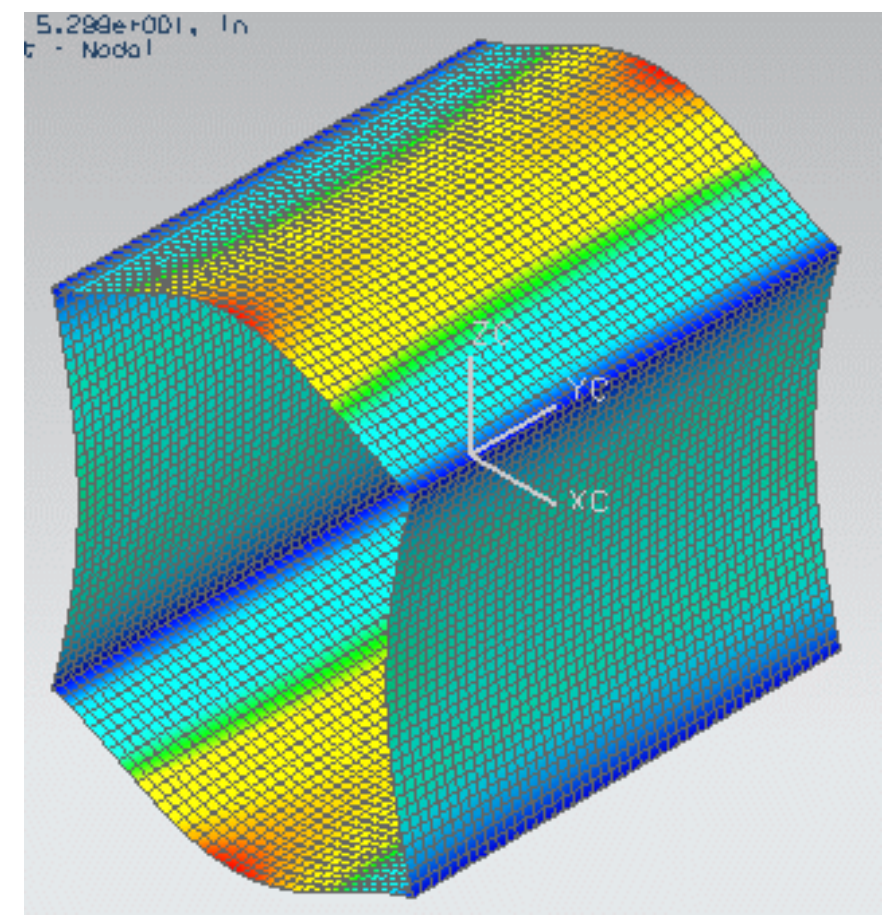

Figure 94: Mode 3 at 1,049 Hz. 


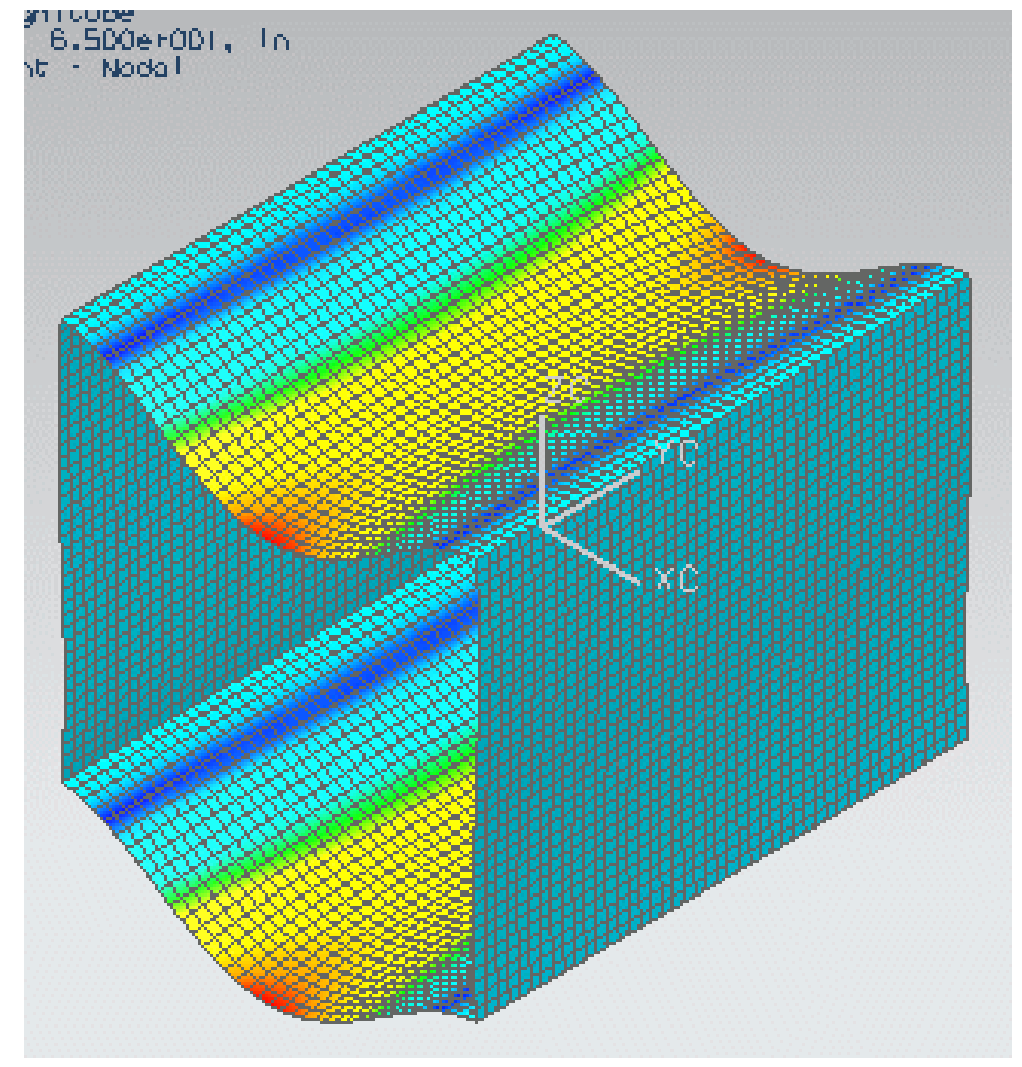

Figure 95: Mode 5 at 1,443 Hz.

Page 132 


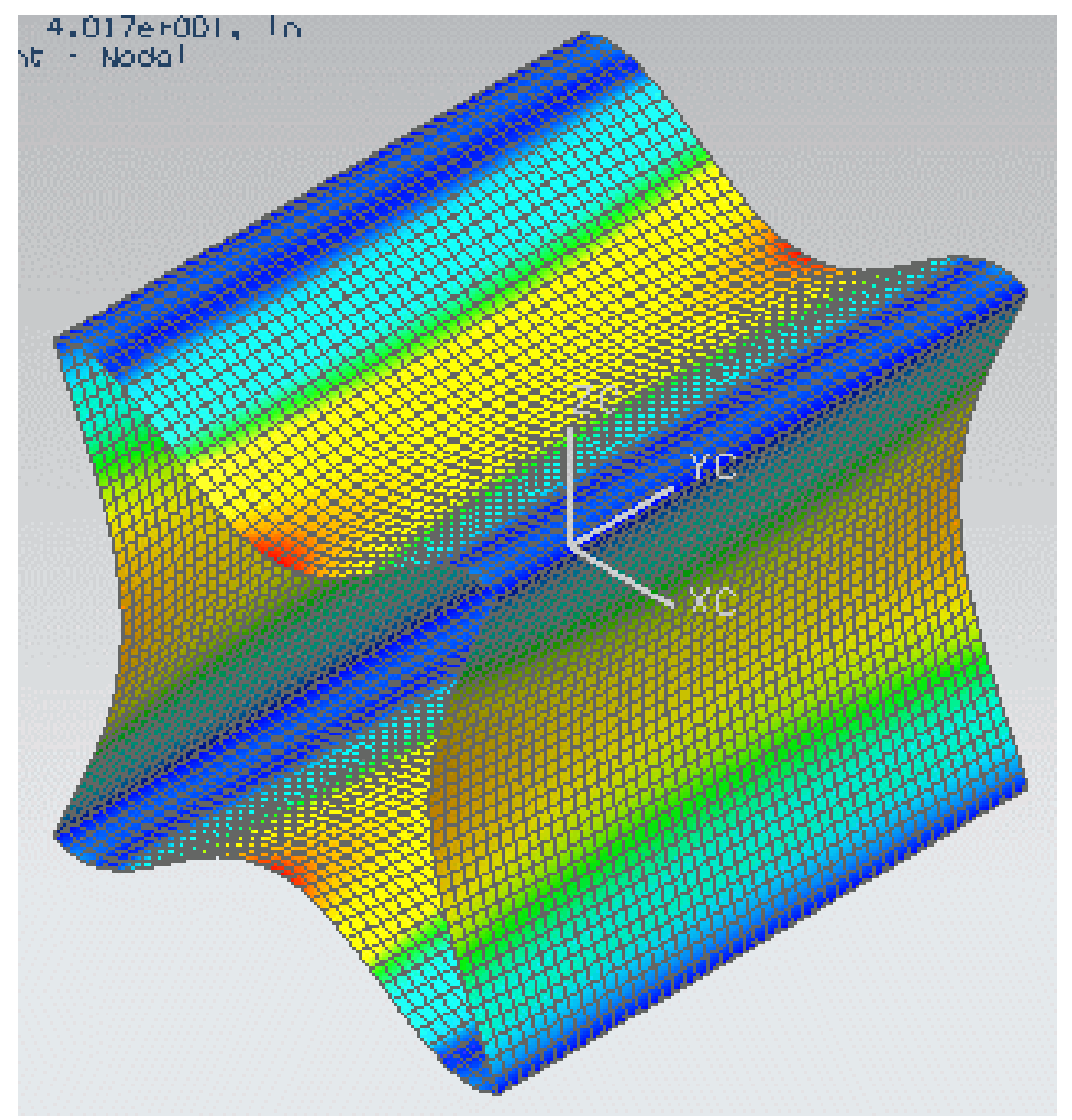

Figure 96: Mode 7 at $1,771 \mathrm{~Hz}$.

A convergence study was run to ensure enough degree of freedom were used to model the Test CubeSat. Figure 97 shows that, at 6930 degrees of freedom, plenty of elements were used in the FEA model. 


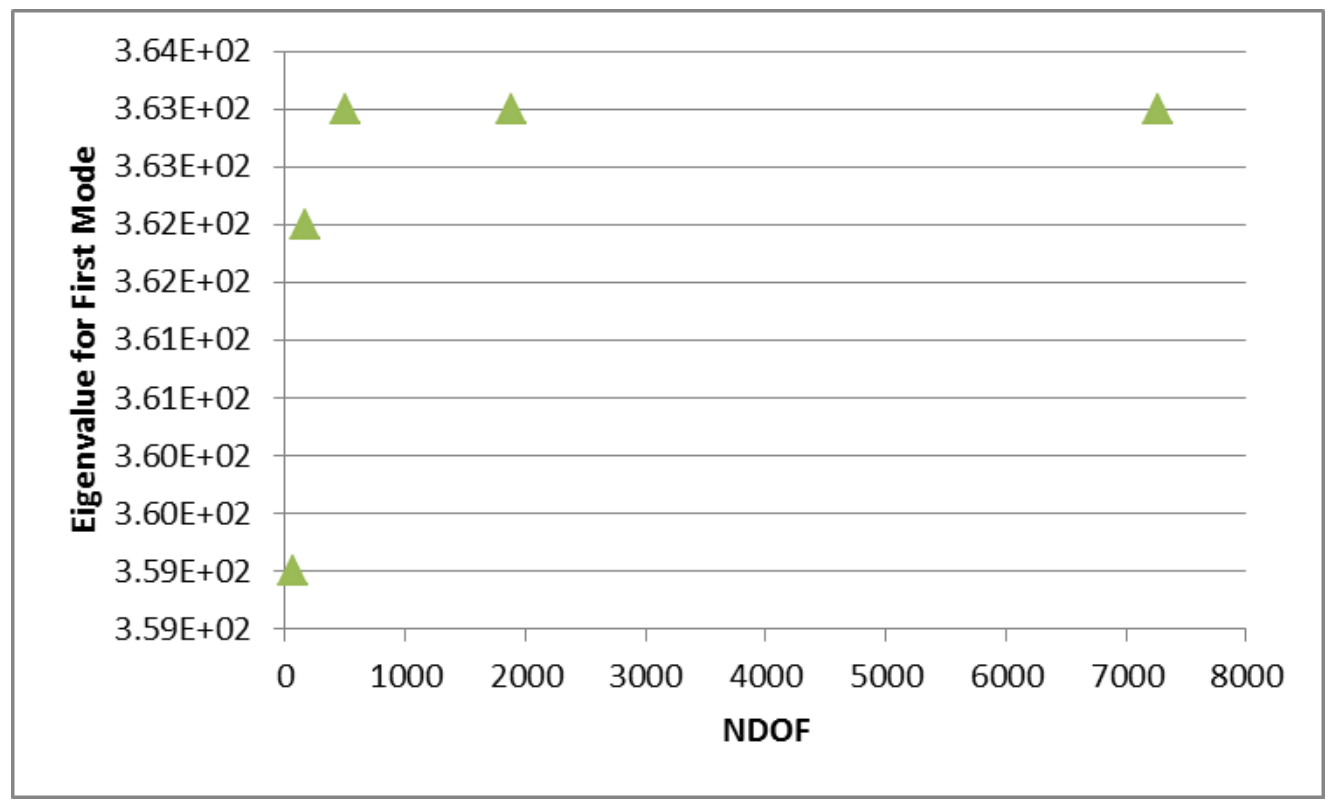

Figure 97: Convergence study for FEM of Test CubeSat.

This study shows that there are resonant frequencies in the $800-2000 \mathrm{~Hz}$ range, and the peaks during testing of the Test CubeSat can be attributed to CubeSat dynamics. 


\section{APPENDIX D: Vibration Test Setup and Data from Chapter 3}

Photos for every vibration setup in Chapter 3 are shown in this appendix. Plots

of the raw data are shown for each test in Chapter 3 as well. Only one screen shot of the raw data was taken per test, so for the tests where the data changed over time, the Microsoft excel data is shown as well. 


\begin{tabular}{|c|c|c|c|c|c|}
\hline $\begin{array}{c}\text { Test } \\
\#\end{array}$ & Deployer & Axis & $\begin{array}{l}\text { Response } \\
\text { Accel. } \\
\text { Location }\end{array}$ & Profile & Spring Plungers \\
\hline 1 & 1U TestPOD & $\mathrm{Z}$ & Deployer & Sine & Standard Integration \\
\hline 2 & 1U TestPOD & $\mathrm{Z}$ & Deployer & Random & Standard Integration \\
\hline 3 & 1U TestPOD & $\mathrm{Z}$ & CubeSat & Random & Standard Integration \\
\hline 4 & 1U TestPOD & $\mathrm{Z}$ & CubeSat & Random & Bolts: Backed Out 1.5 Turns \\
\hline 5 & 1U TestPOD & $\mathrm{X}$ & Deployer & Sine & Standard Integration \\
\hline 6 & 1U TestPOD & $\mathrm{X}$ & Deployer & Random & Standard Integration \\
\hline 7 & 1U TestPOD & $\mathrm{X}$ & CubeSat & Random & Standard Integration \\
\hline $8-1$ & 1U TestPOD & $\mathrm{X}$ & CubeSat & Random & Dialed in Tight \\
\hline $8-2$ & 1U TestPOD & $\mathrm{X}$ & CubeSat & Sine & Dialed in Tight \\
\hline $9-1$ & 1U TestPOD & $\mathrm{X}$ & CubeSat & Random & Backed Out One Turn \\
\hline $9-2$ & 1U TestPOD & $\mathrm{X}$ & CubeSat & Sine & Backed Out One Turn \\
\hline 10 & 3U TestPOD & $\mathrm{Z}$ & Deployer & Sine & Standard Integration \\
\hline $11-1$ & 3U TestPOD & $\mathrm{Z}$ & Deployer & Random & Standard Integration \\
\hline $12-1$ & 3U TestPOD & $\mathrm{Z}$ & CubeSat & Random & Standard Integration \\
\hline $12-2$ & 3U TestPOD & $\mathrm{Z}$ & CubeSat & Random & Standard Integration \\
\hline 13 & 3U TestPOD & $\mathrm{X}$ & Deployer & Sine & Standard Integration \\
\hline 14 & 3U TestPOD & $\mathrm{X}$ & Deployer & Random & Standard Integration \\
\hline $15-1$ & 3U TestPOD & $\mathrm{X}$ & CubeSat & Random & Standard Integration \\
\hline $15-2$ & 3U TestPOD & $\mathrm{X}$ & CubeSat & Random & Standard Integration \\
\hline 16 & P-POD & $\mathrm{Z}$ & Deployer & Sine & Standard Integration \\
\hline 17 & P-POD & $\mathrm{Z}$ & Deployer & Random & Standard Integration \\
\hline $18-1$ & P-POD & $\mathrm{Z}$ & CubeSat & Random & Standard Integration \\
\hline $18-2$ & P-POD & $\mathrm{Z}$ & CubeSat & Random & Standard Integration \\
\hline $19-1$ & P-POD & $\mathrm{Z}$ & CubeSat & Random 2 & Standard Integration \\
\hline 20 & P-POD & $\mathrm{X}$ & Deployer & Sine & Standard Integration \\
\hline 21 & P-POD & $\mathrm{X}$ & Deployer & Random & Standard Integration \\
\hline $22-1$ & P-POD & $\mathrm{X}$ & CubeSat & Random & Standard Integration \\
\hline $22-2$ & P-POD & $\mathrm{X}$ & CubeSat & Random & Standard Integration \\
\hline $23-1$ & P-POD & $\mathrm{X}$ & CubeSat & Random 2 & Standard Integration \\
\hline 24 & P-POD & $\mathrm{Y}$ & Deployer & Sine & Standard Integration \\
\hline 25 & P-POD & $\mathrm{Y}$ & Deployer & Random & Standard Integration \\
\hline $26-1$ & P-POD & $\mathrm{Y}$ & CubeSat & Random & Standard Integration \\
\hline $26-2$ & P-POD & $\mathrm{Y}$ & CubeSat & Random & Standard Integration \\
\hline $27-1$ & P-POD & $\mathrm{Y}$ & CubeSat & Random 2 & Standard Integration \\
\hline
\end{tabular}




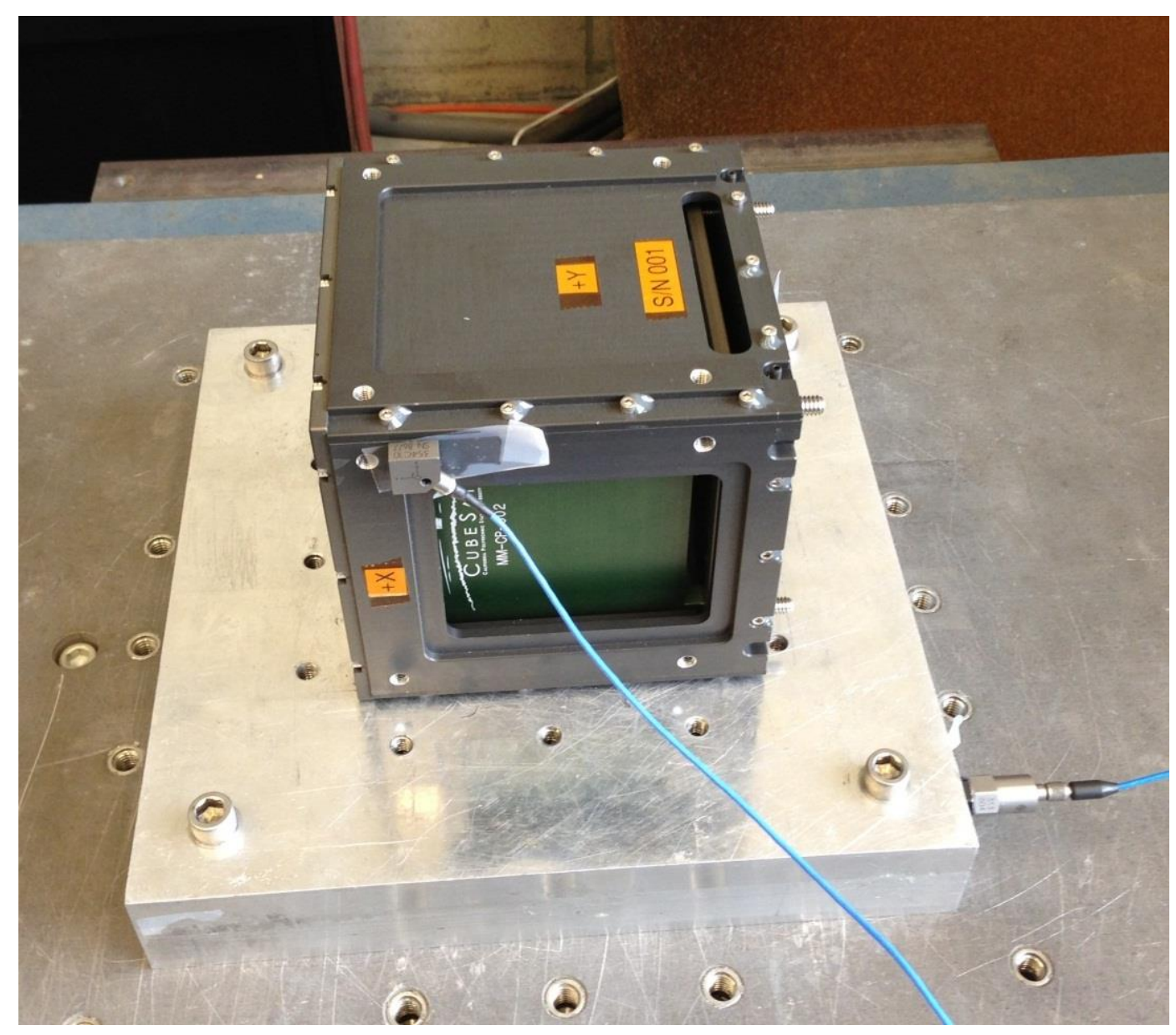

Figure 98: Setup for test numbers 1 and 2. 


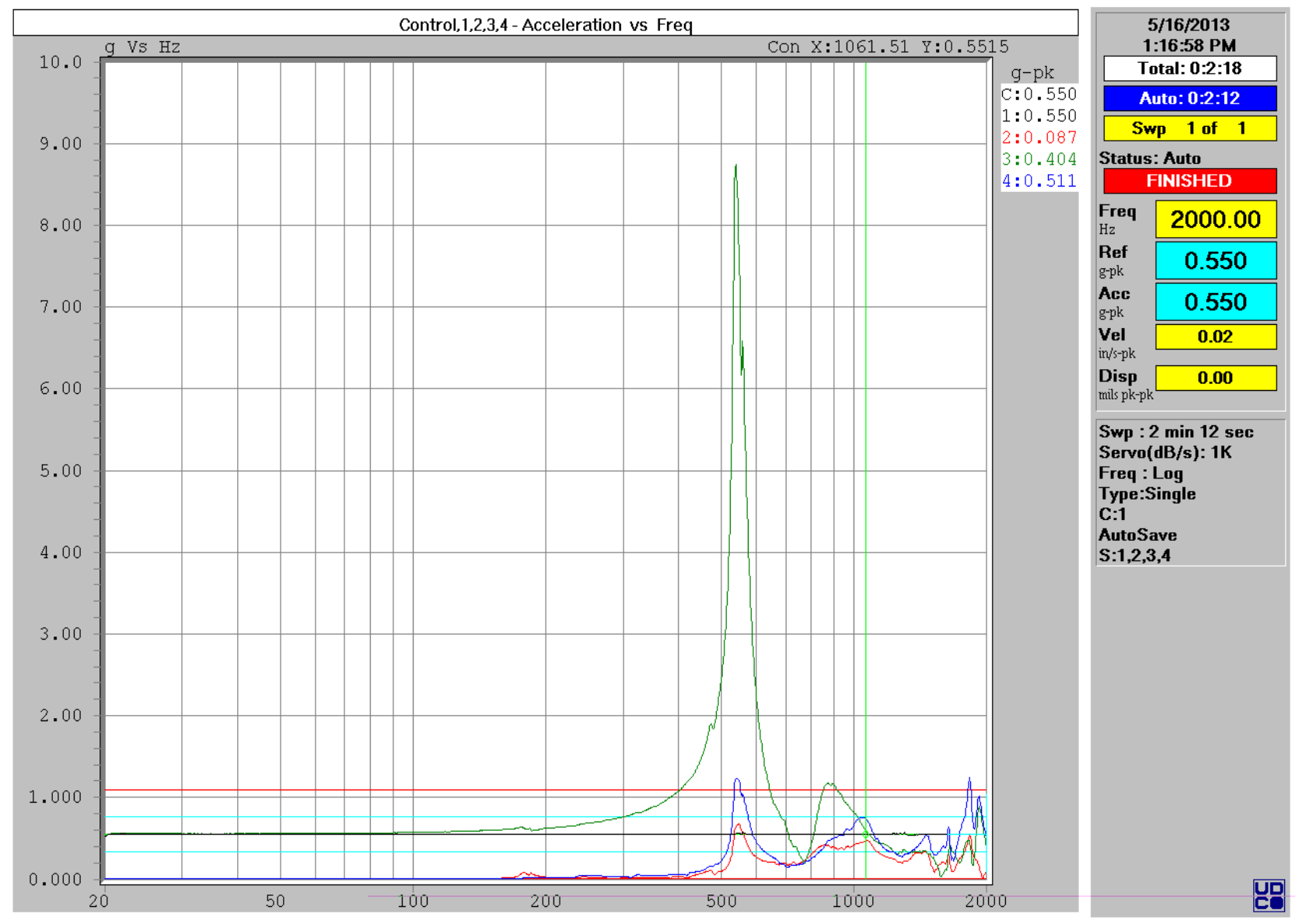

Figure 99: Raw data from test number 1 


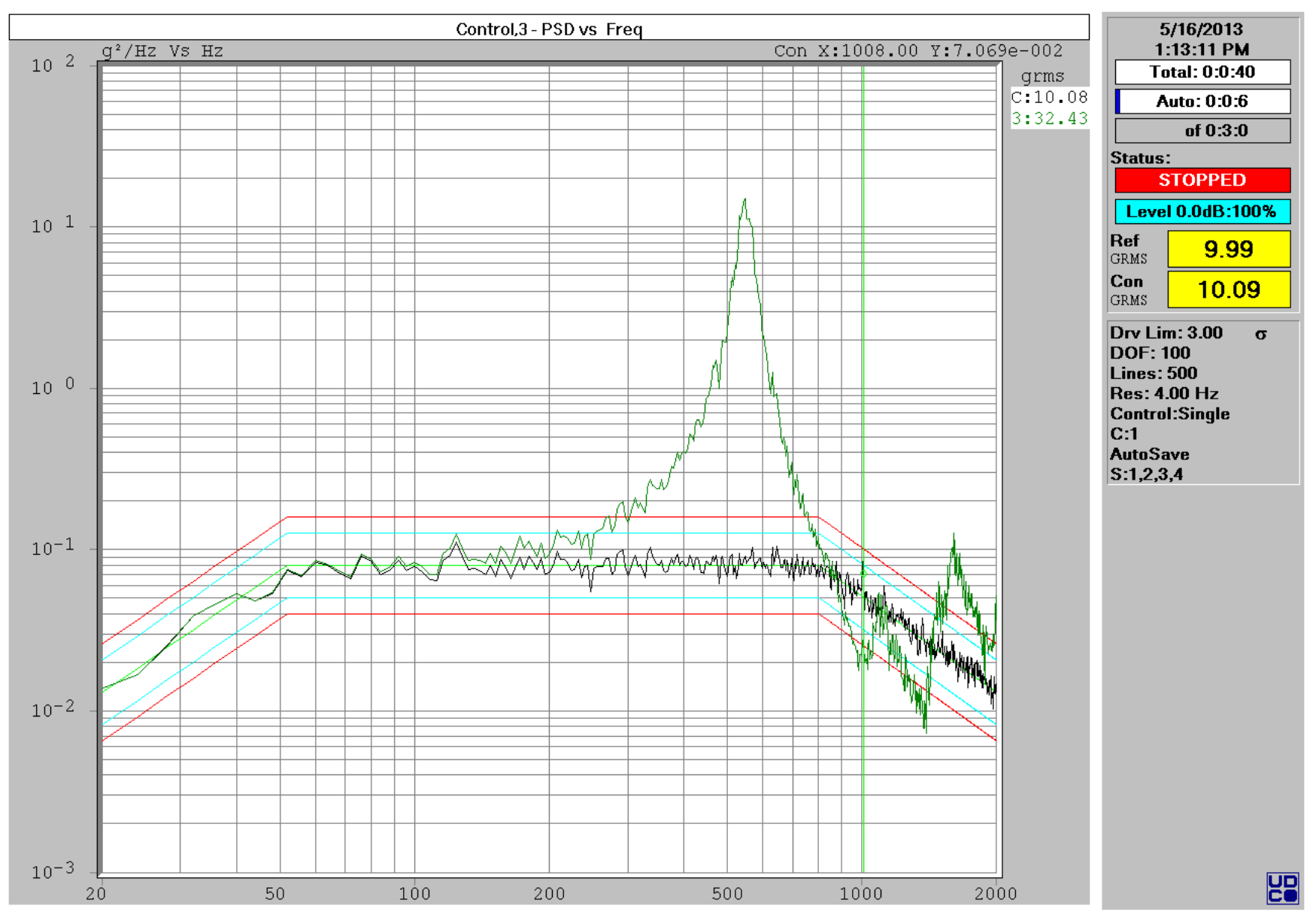

Figure 100: Raw data from test number 2. 


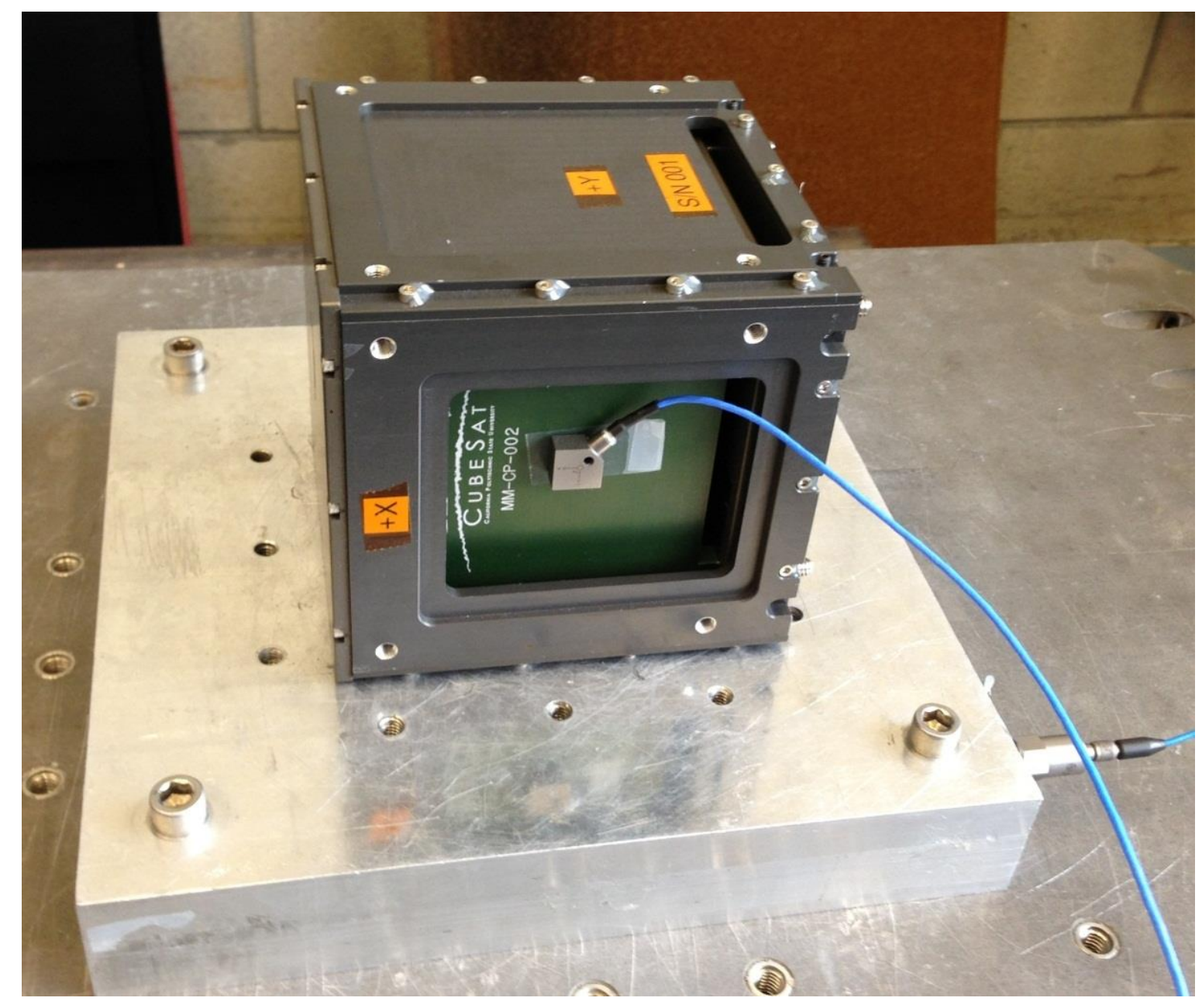

Figure 101: Setup for test number 3. 


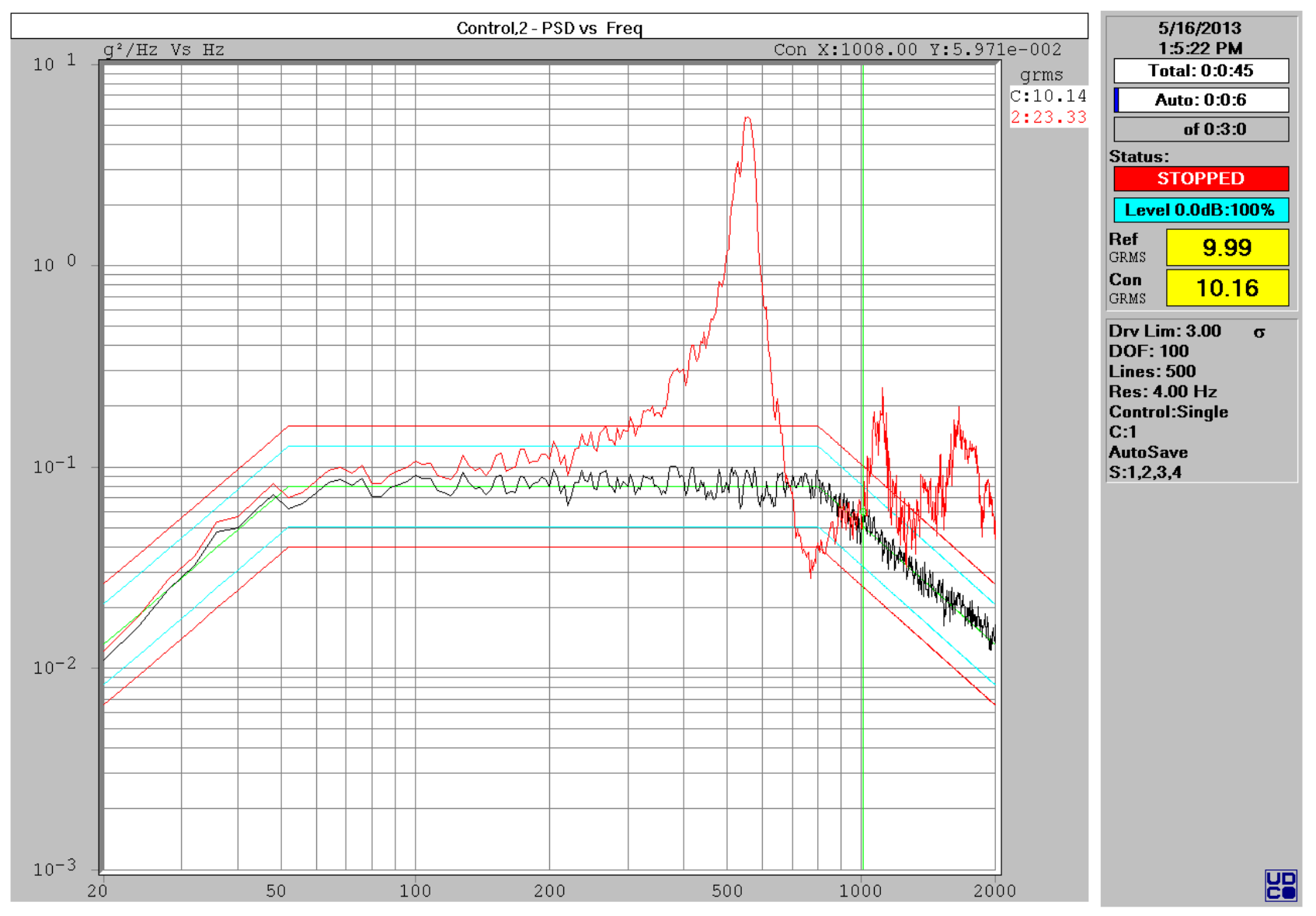

Figure 102: Raw data from test 3. 


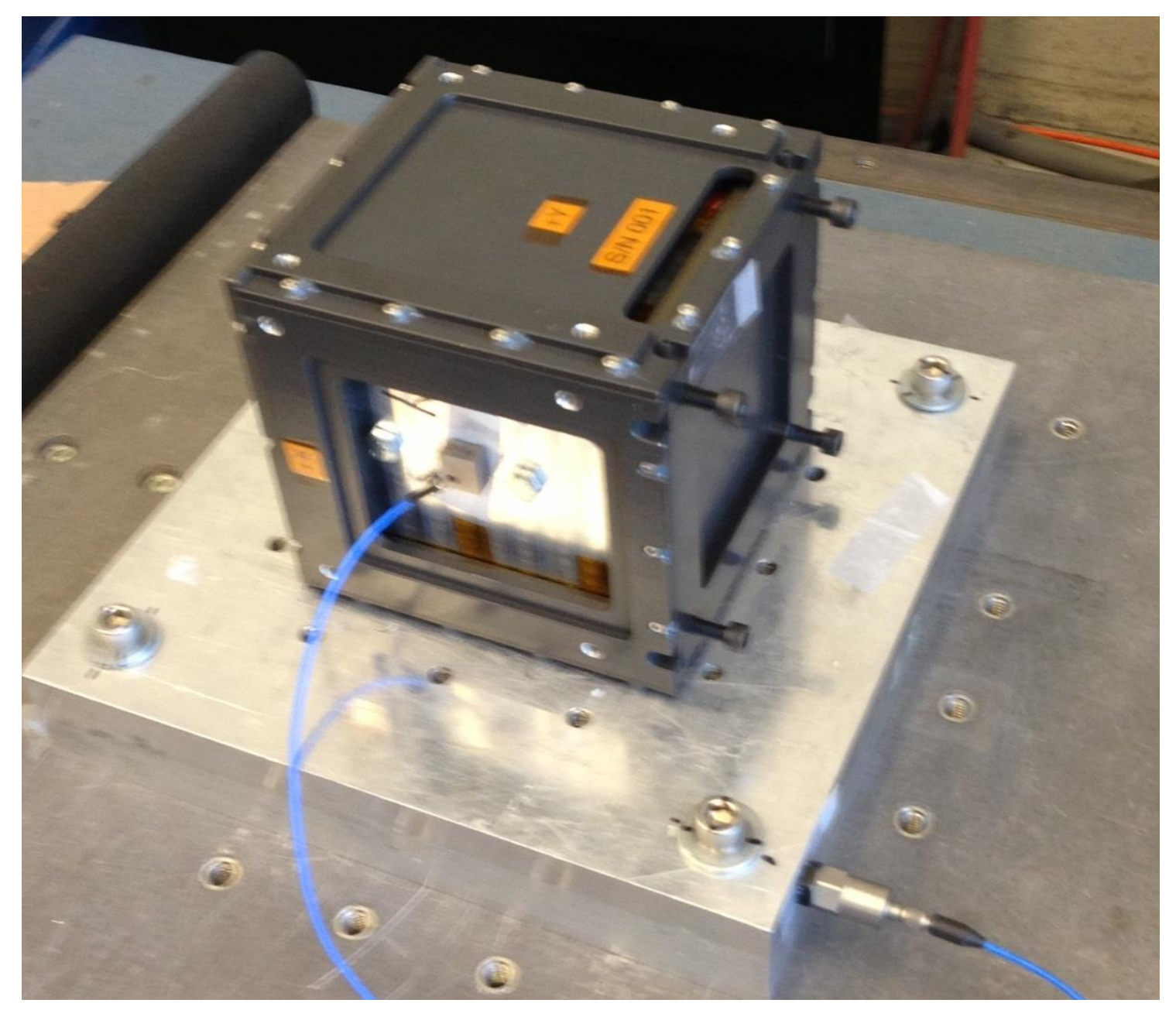

Figure 103: Setup for test 4. 

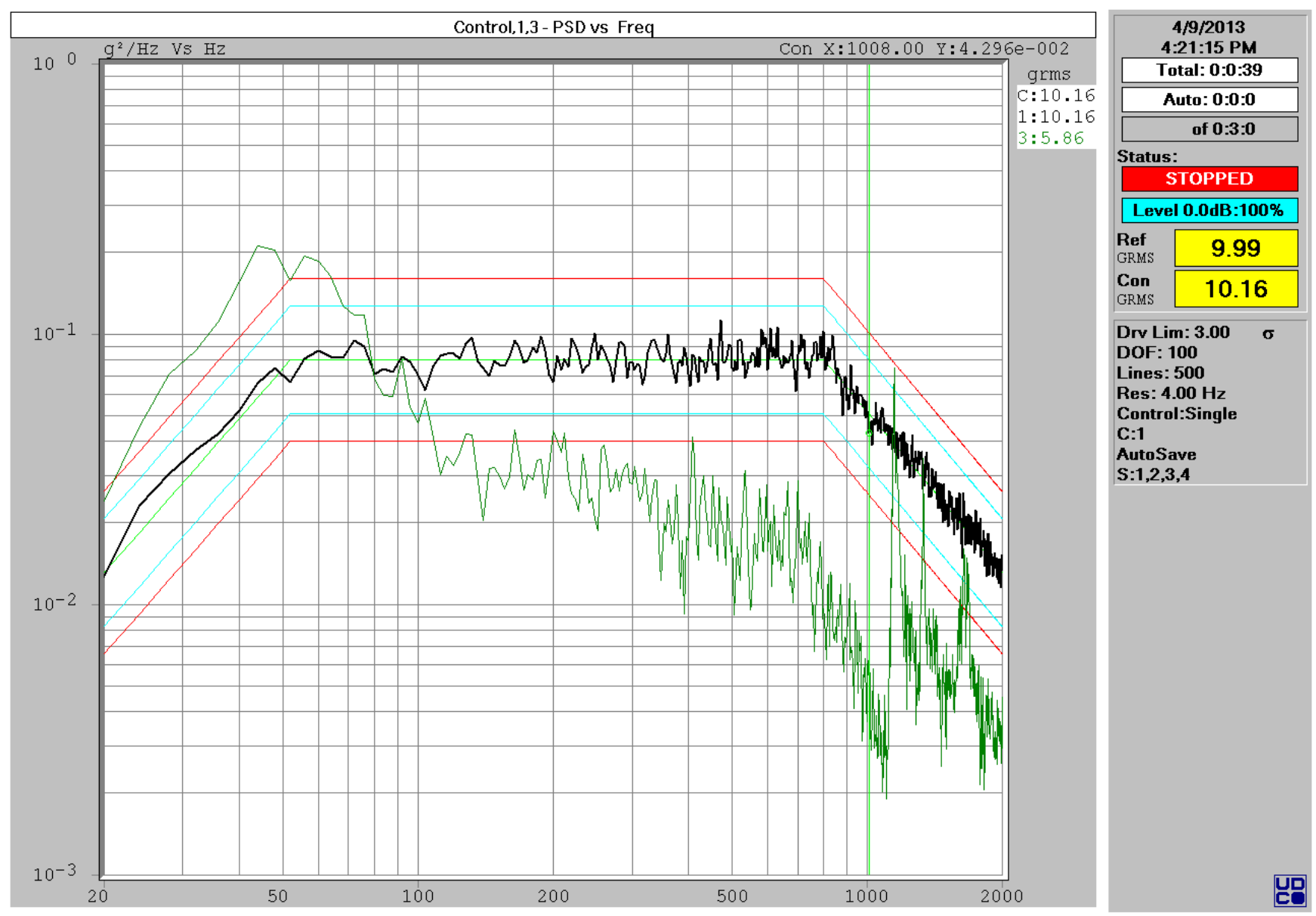

Figure 104: Raw data from test 4. 


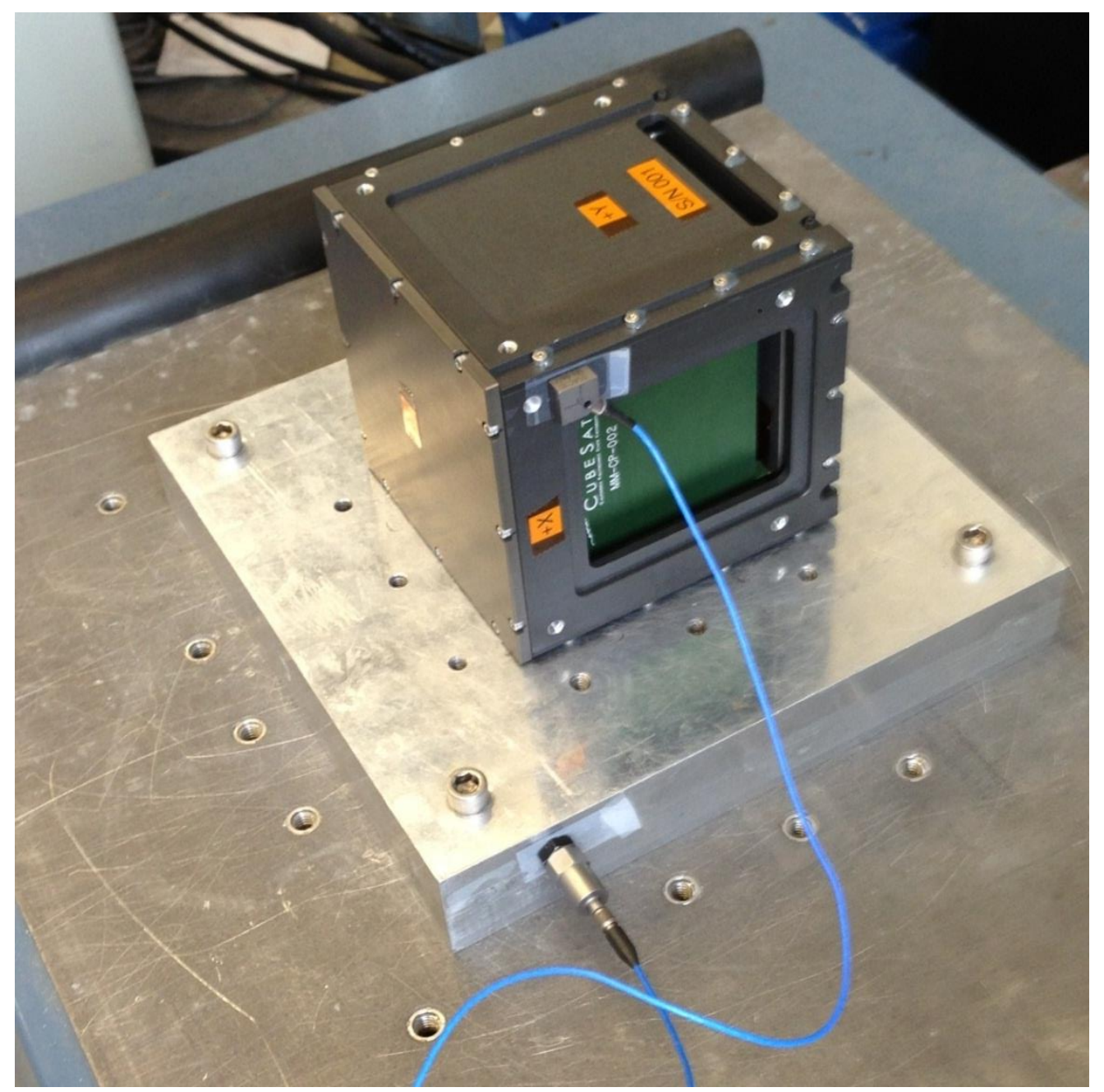

Figure 105: Setup for tests 5 and 6. 


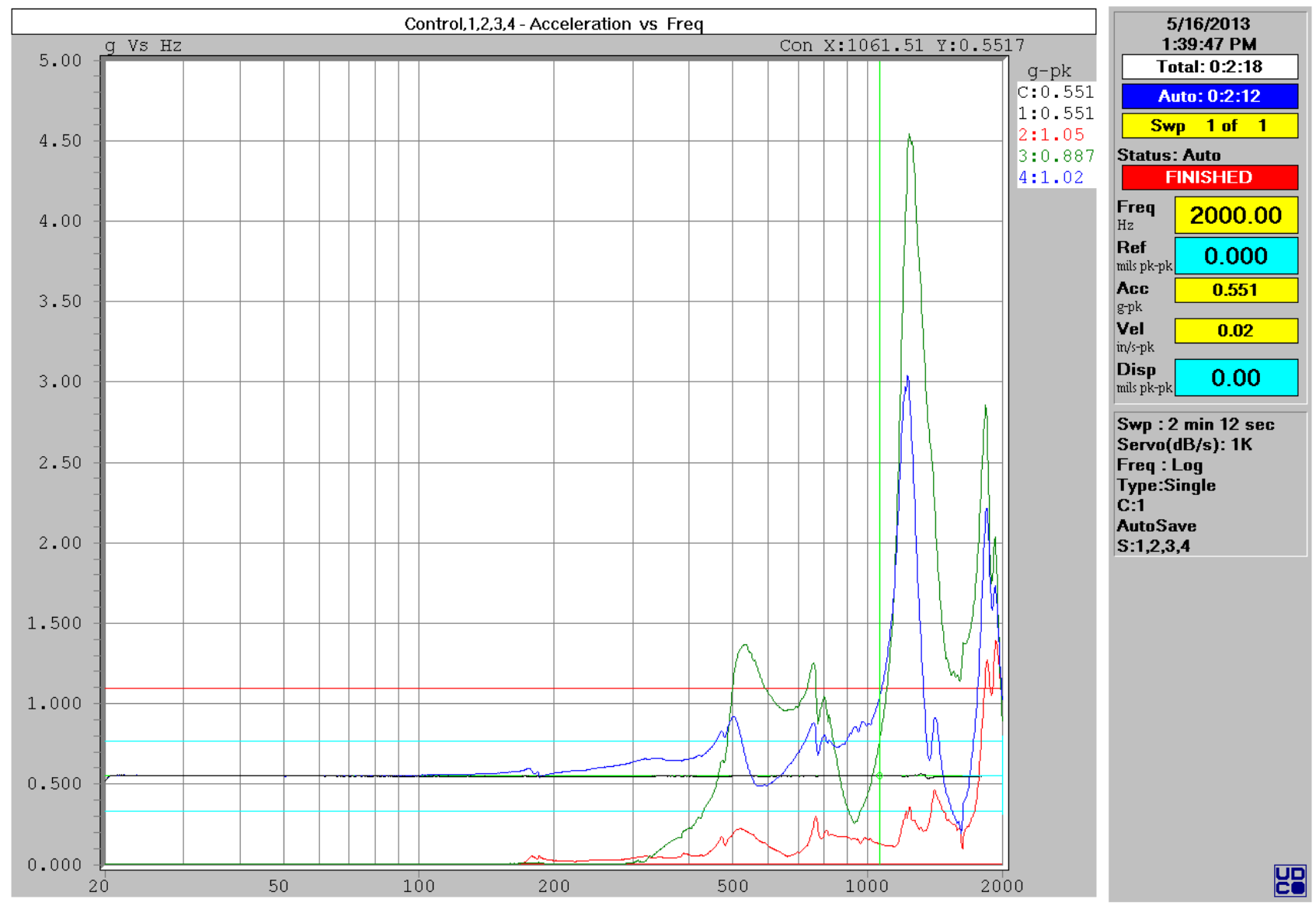

Figure 106: Raw data from test number 5. 


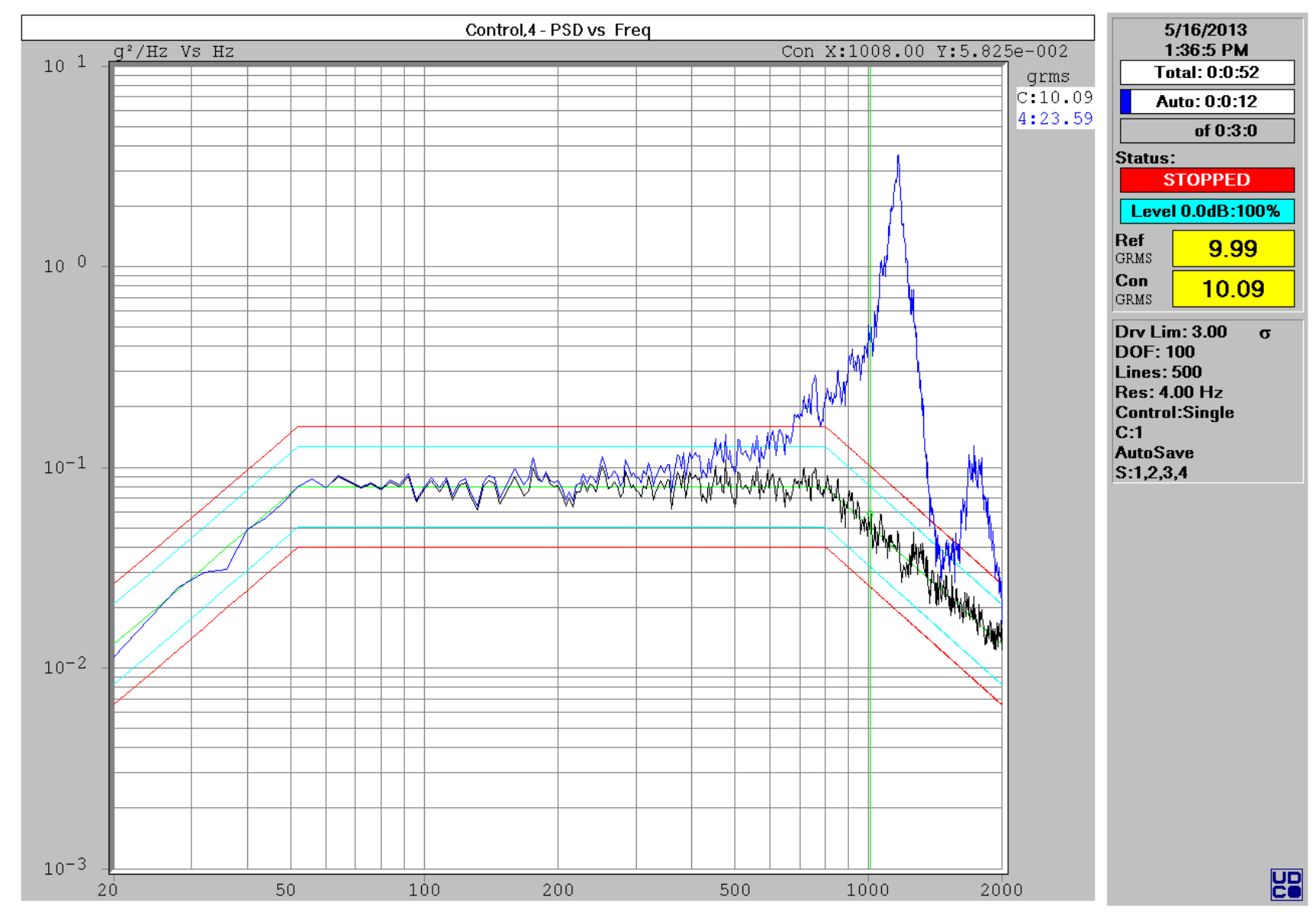

Figure 107: Raw data from test 6. 


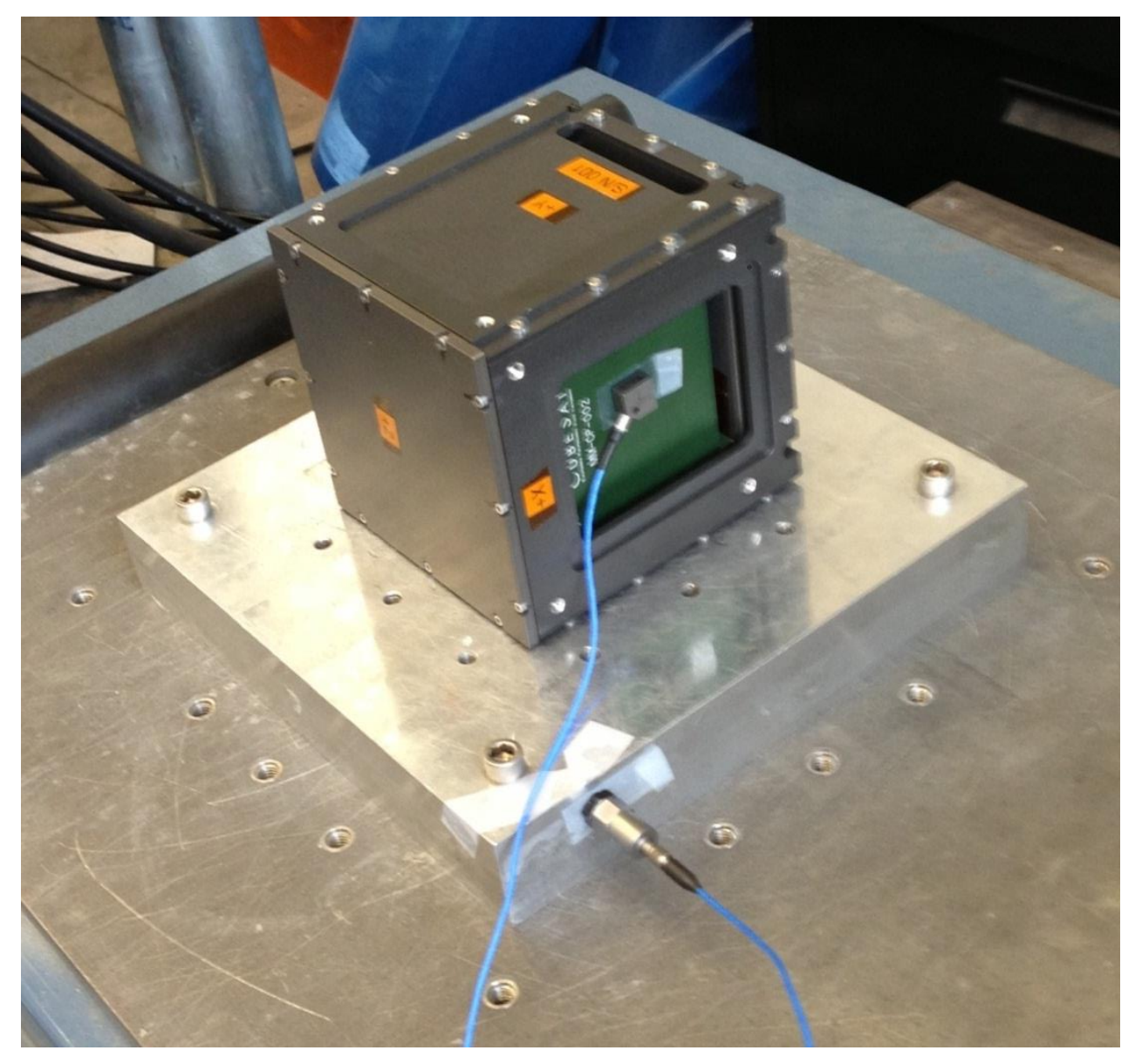

Figure 108: Setup for tests 7, 8-1, 8-2, 9-1, and 9-2 (spring plungers were dialed in differently for these tests, but cannot be distinguished through photos). 

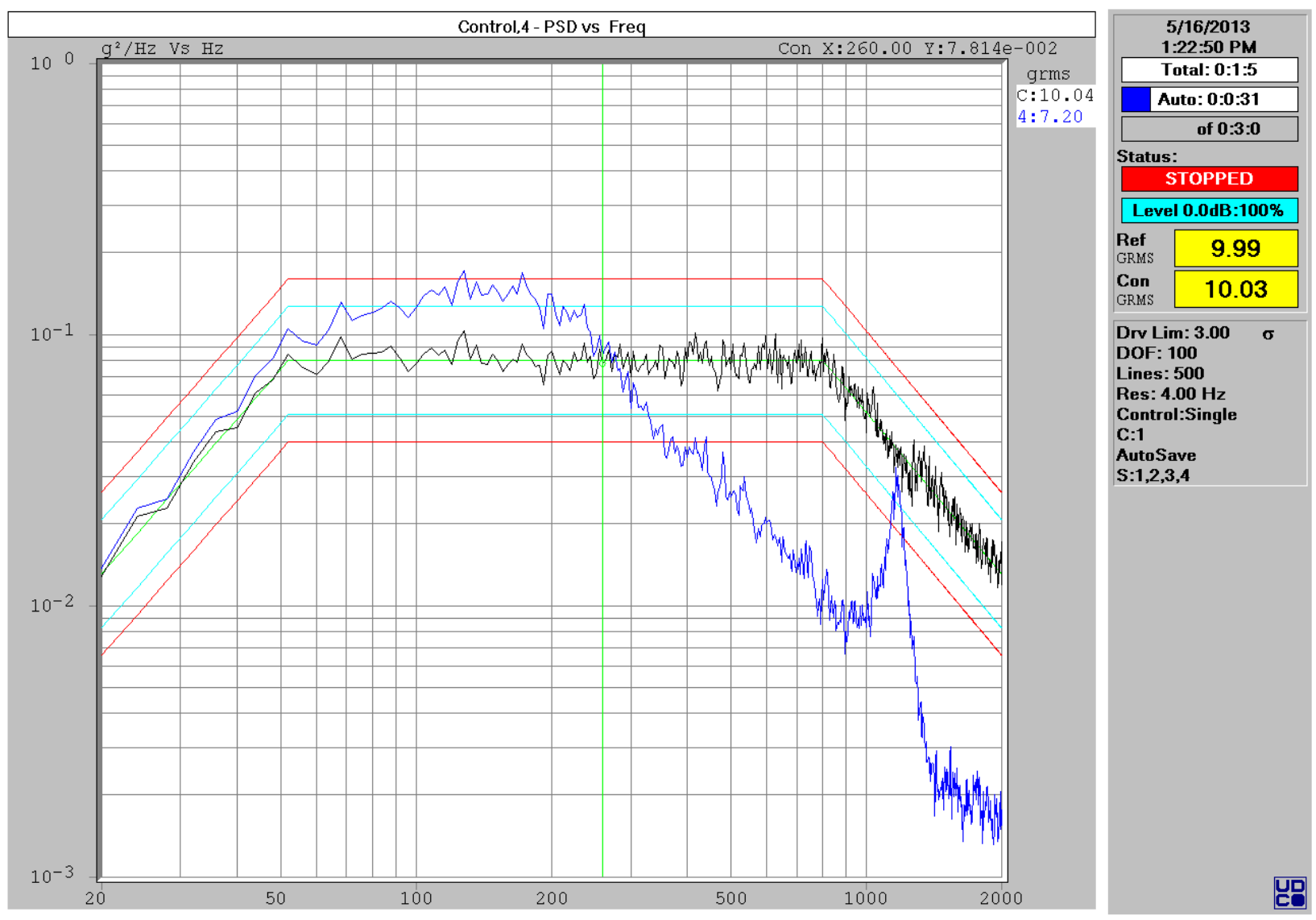

Figure 109: Raw data from test number 7. 


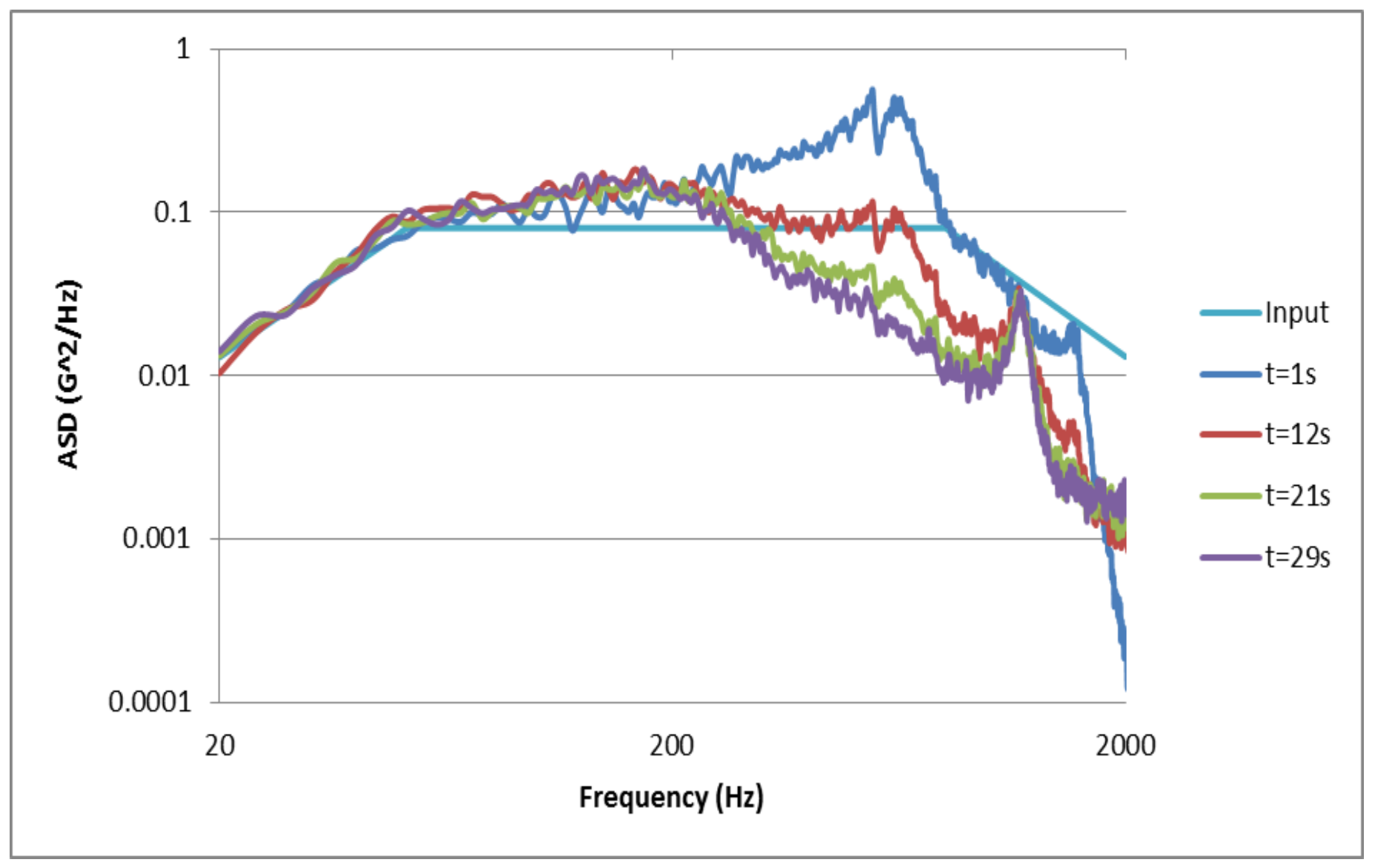

Figure 110: Time varying data from test number 7. 


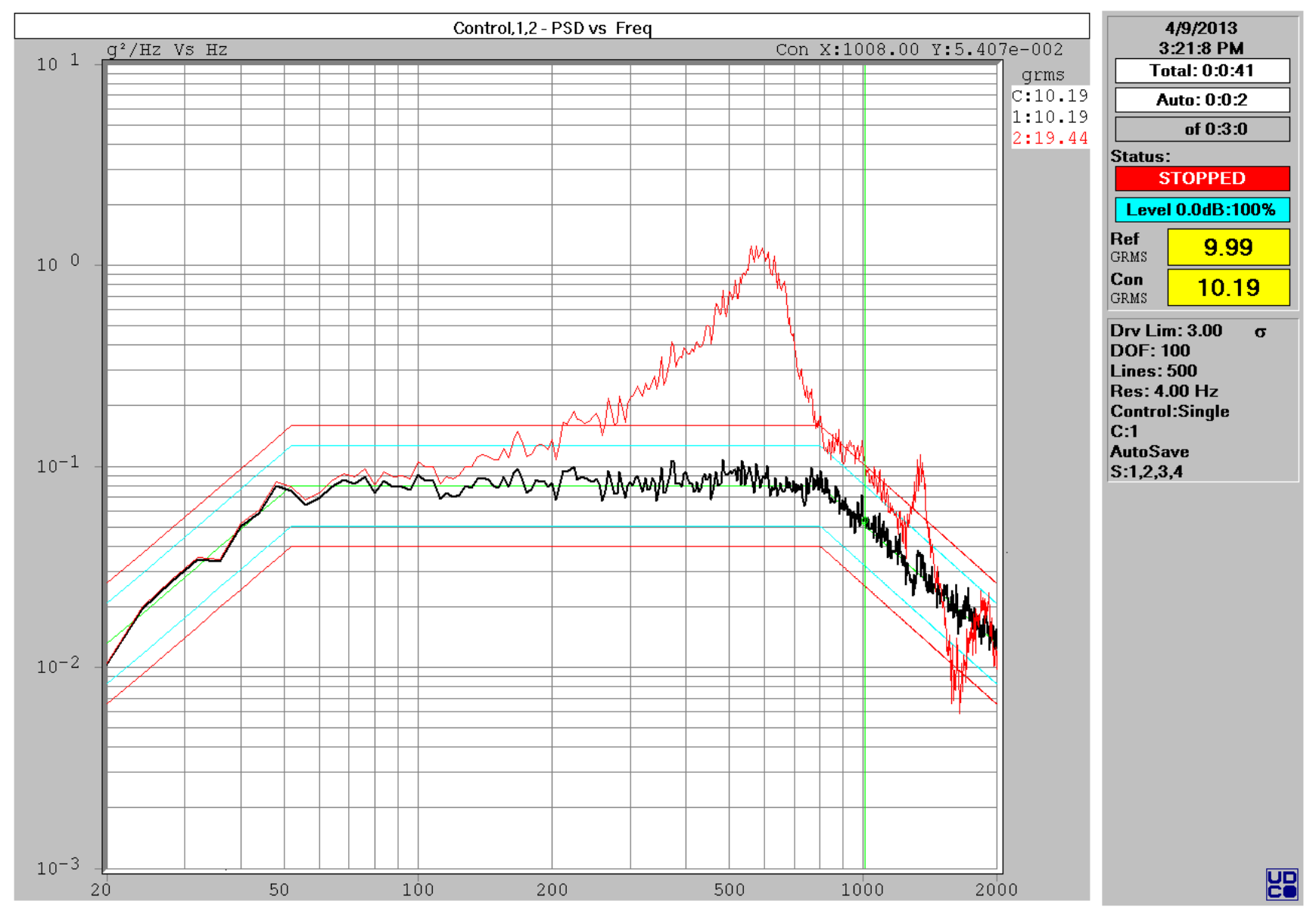

Figure 111: Raw data from test number 8-1. 

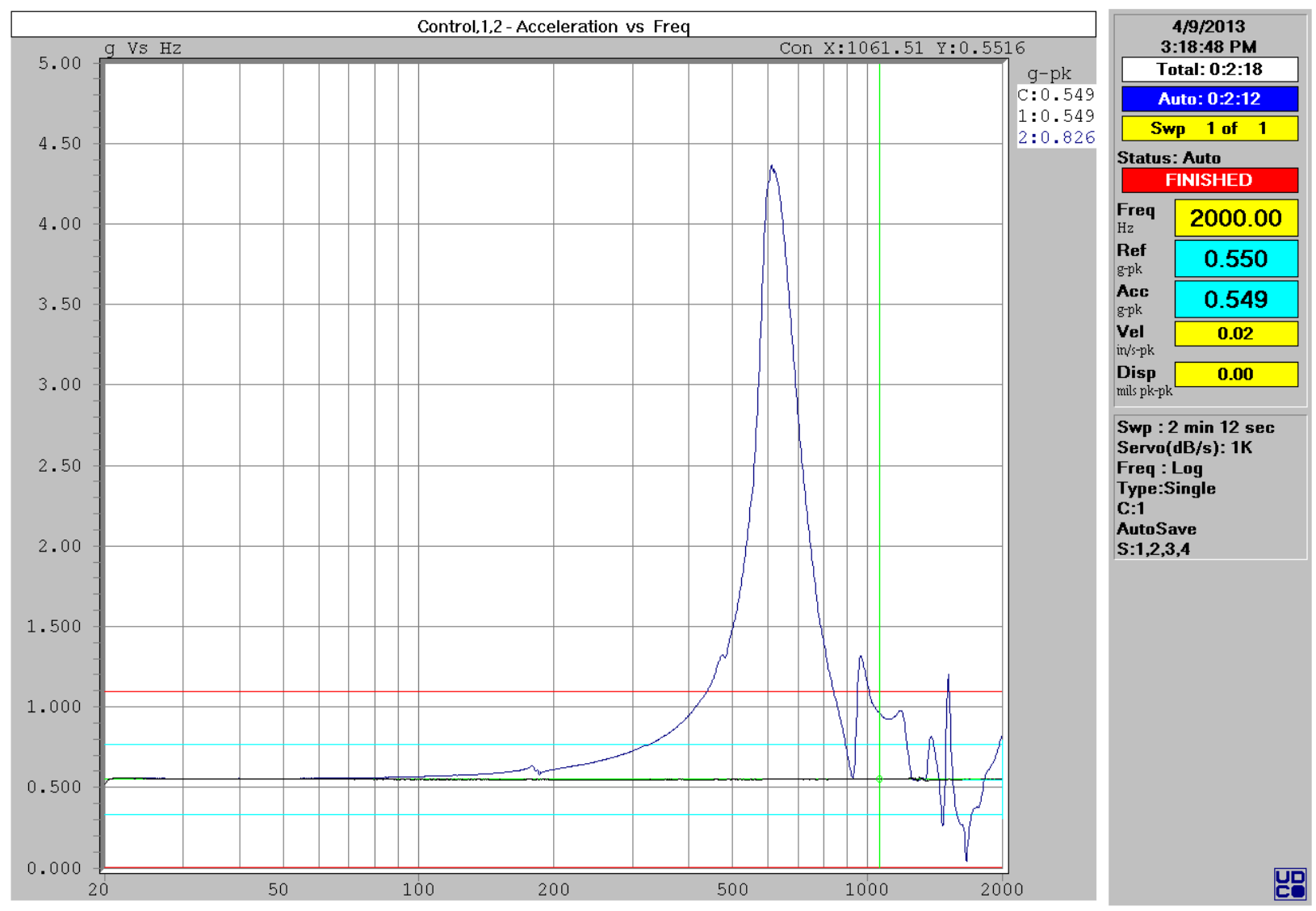

Figure 112: Raw data from test number 8-2. 


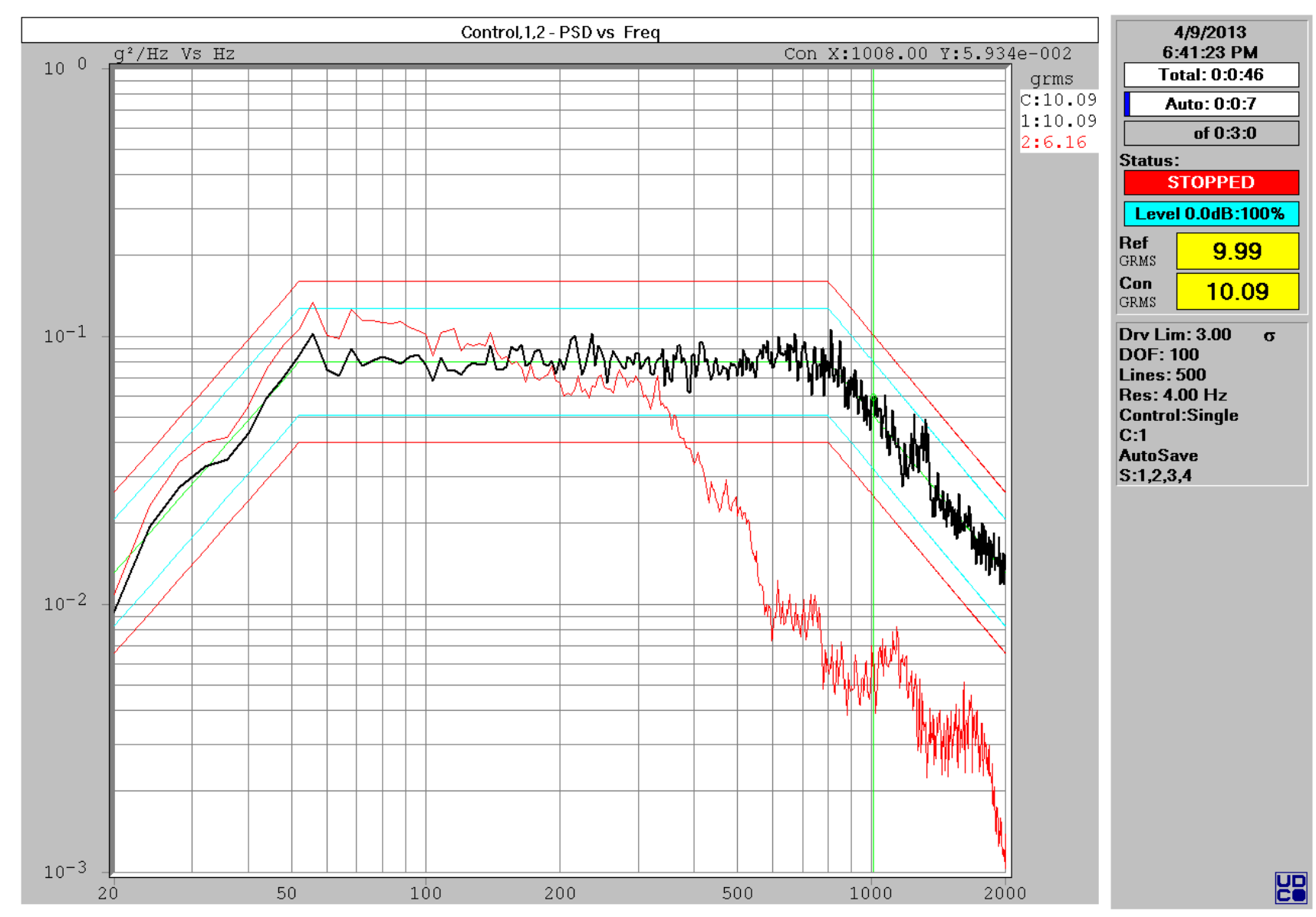

Figure 113: Raw data from test number 9-1. 


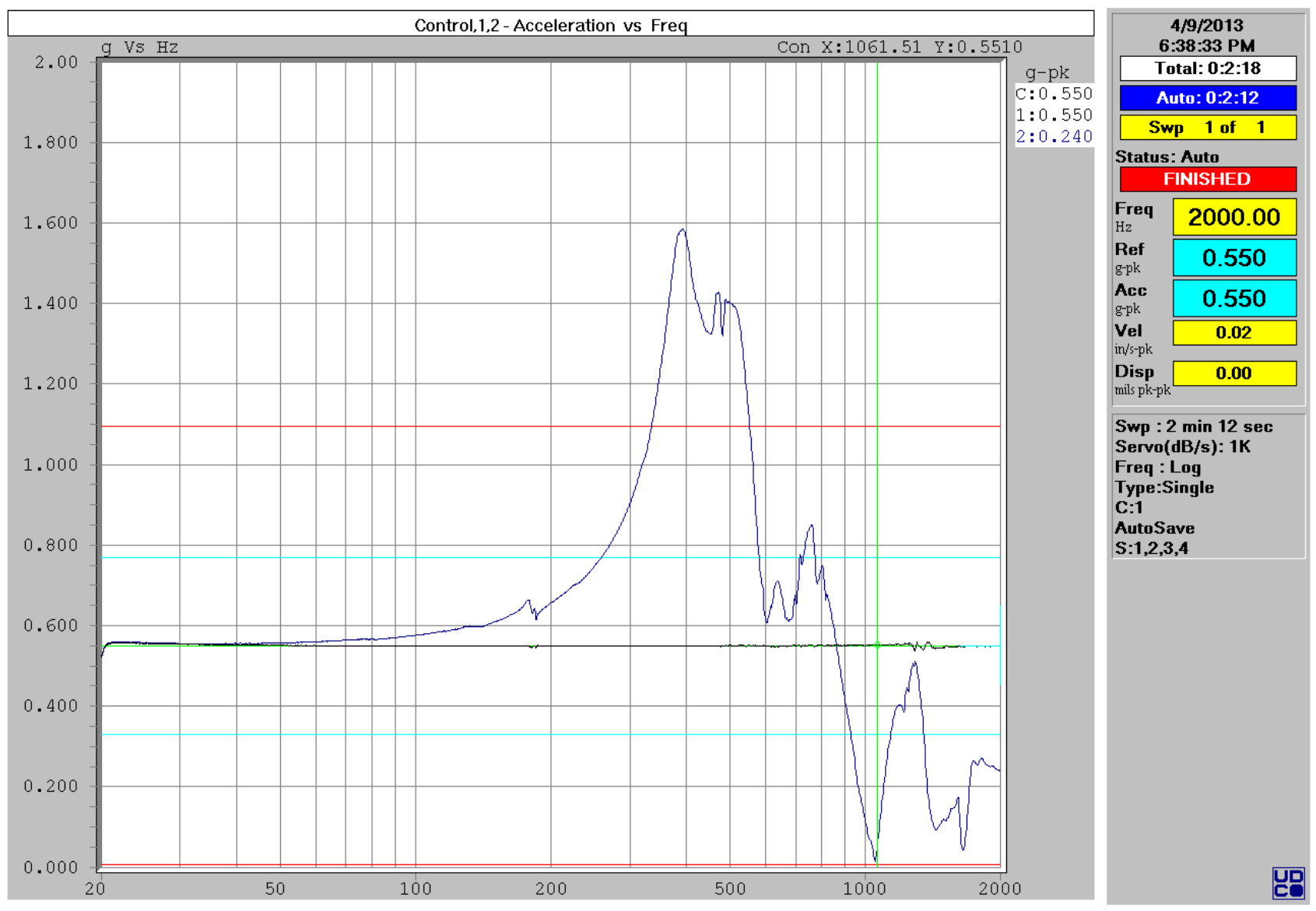

Figure 114: Raw data from test number 9-2. 


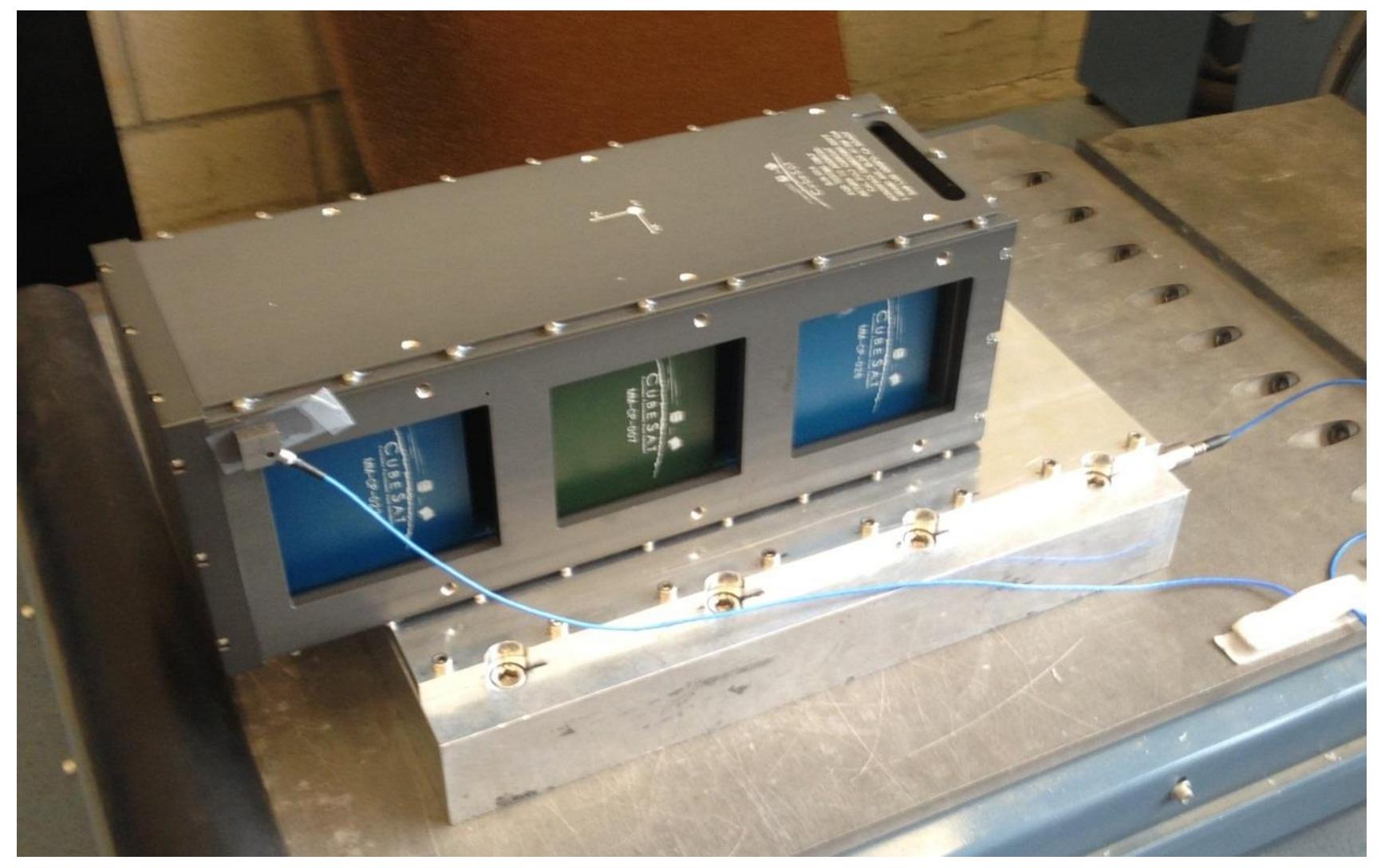

Figure 115: Setup for tests 10 and 11. 

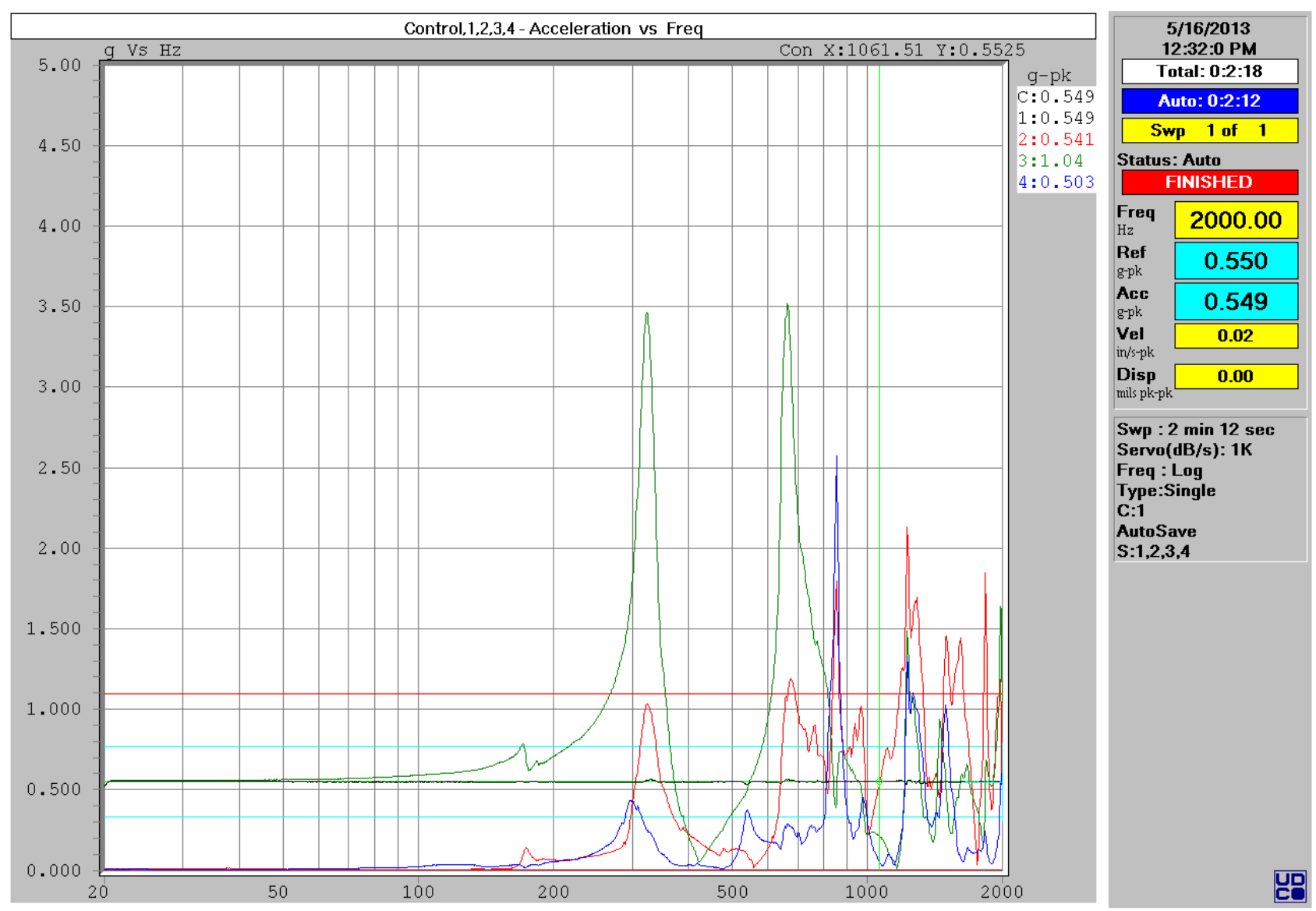

Figure 116: Raw data from test 10. 


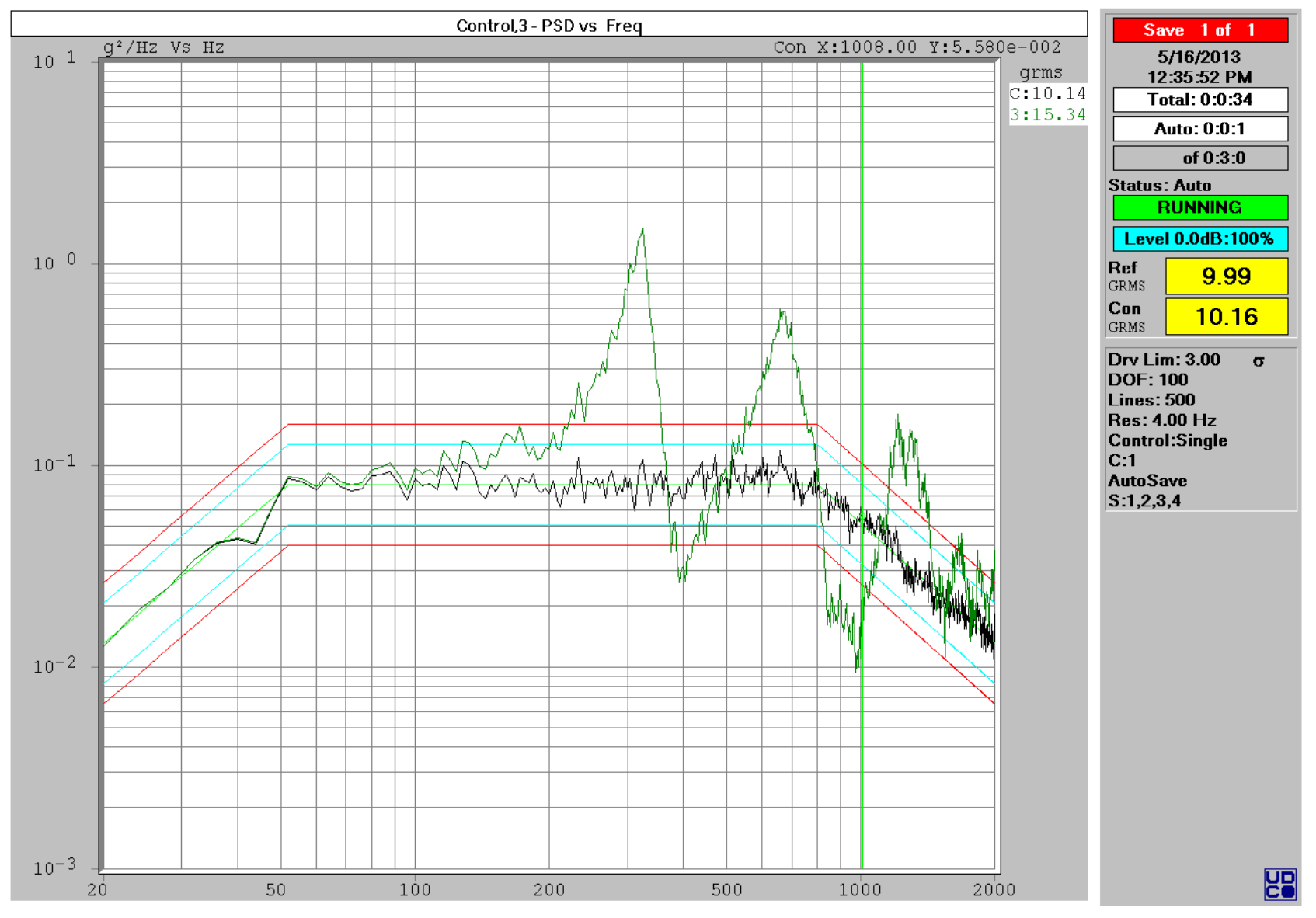

Figure 117: Raw data from test 11. 


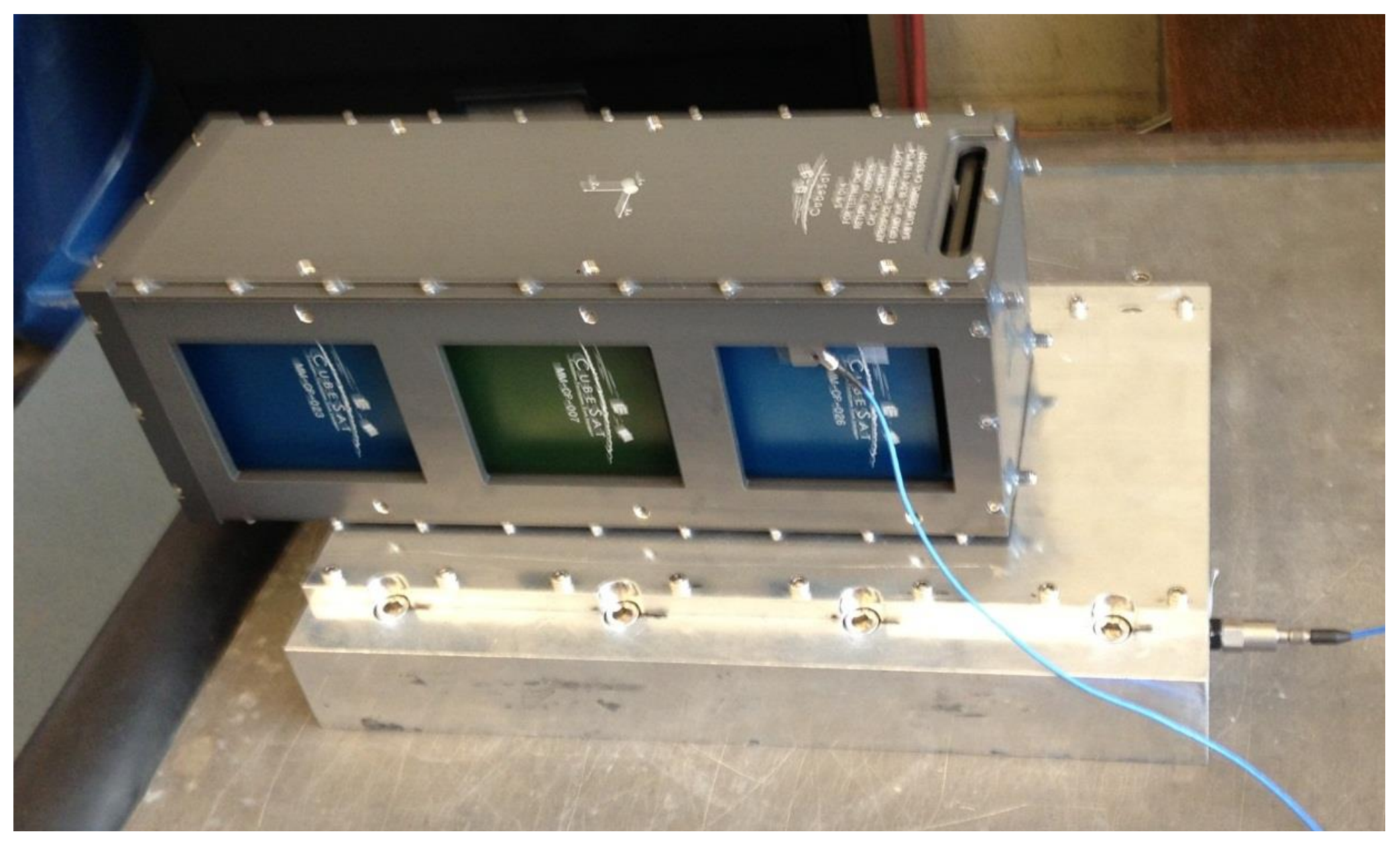

Figure 118: Setup for test 12-1. 


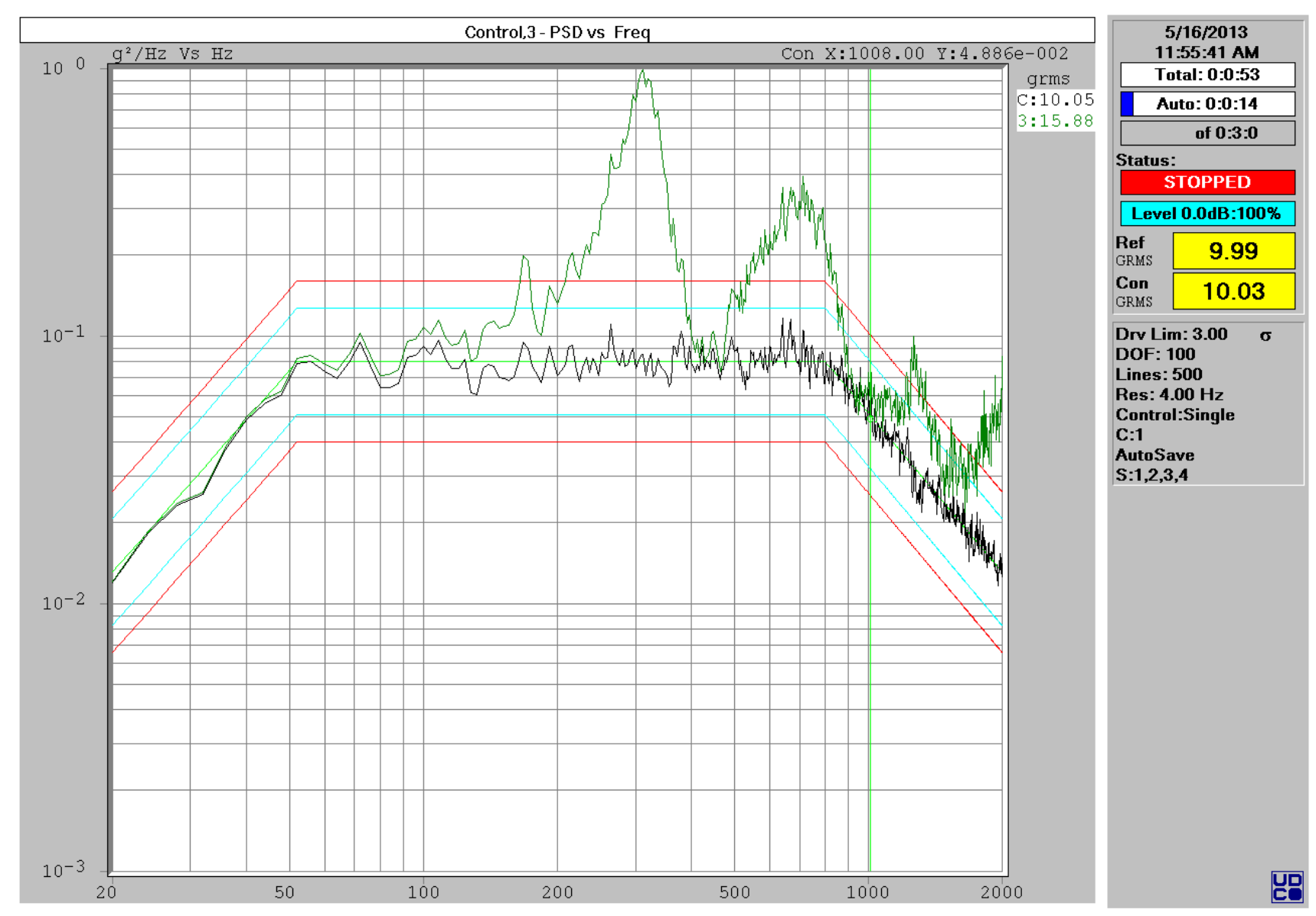

Figure 119: Raw data from test 12-1. 


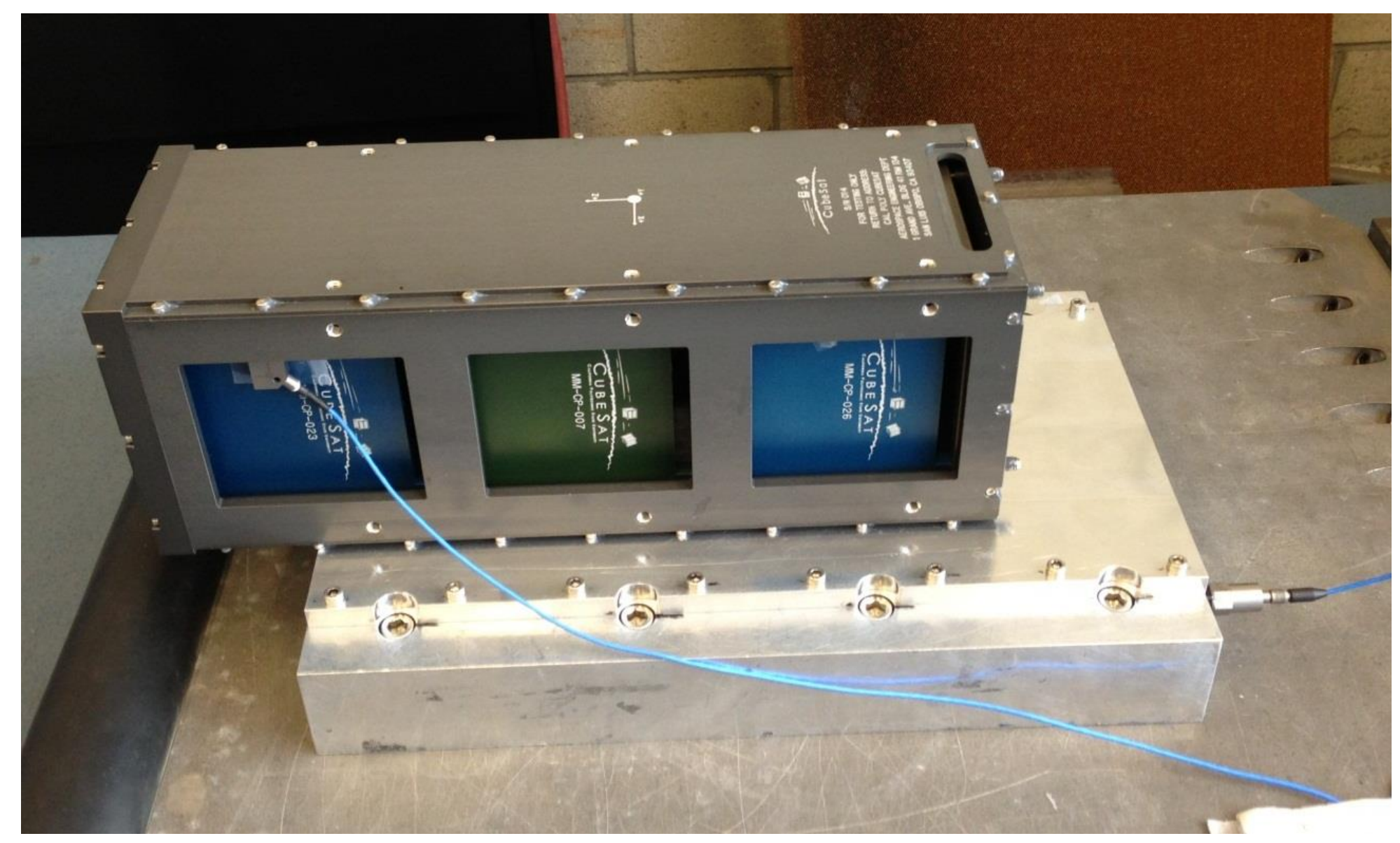

Figure 120: Setup for test 12-2. 

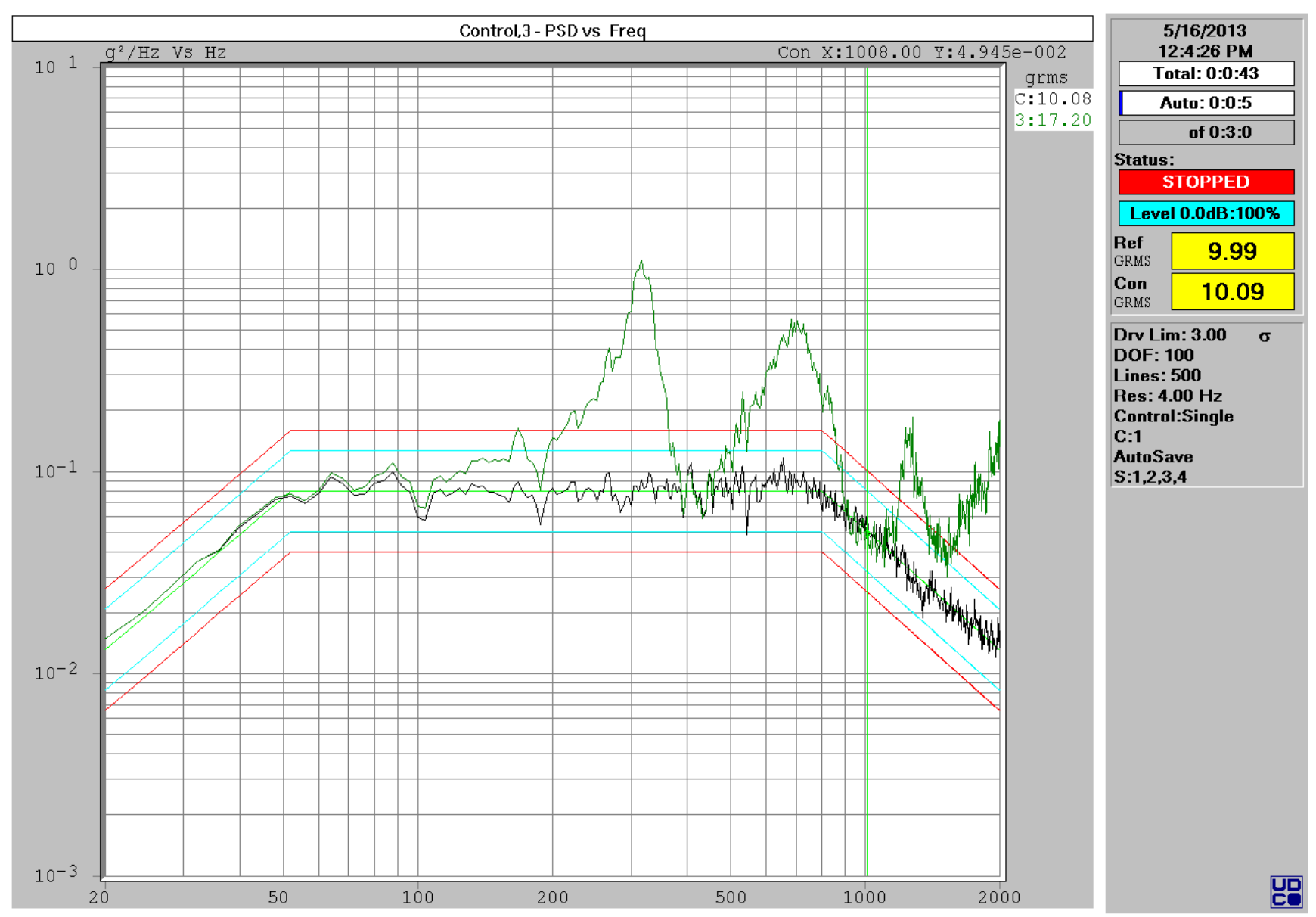

Figure 121: Raw data from test 12-2. 


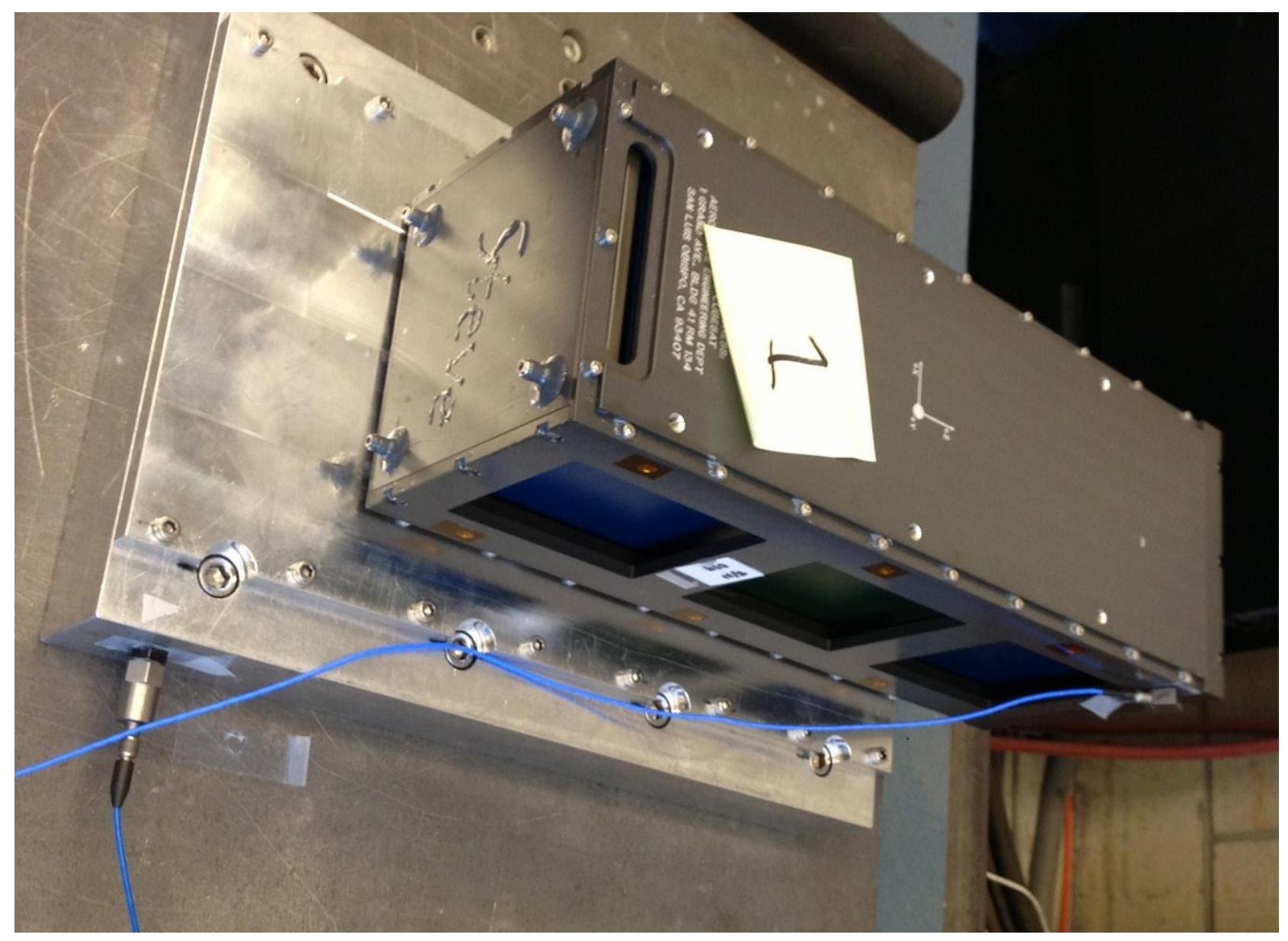

Figure 122: Setup for test 13 and 14. 


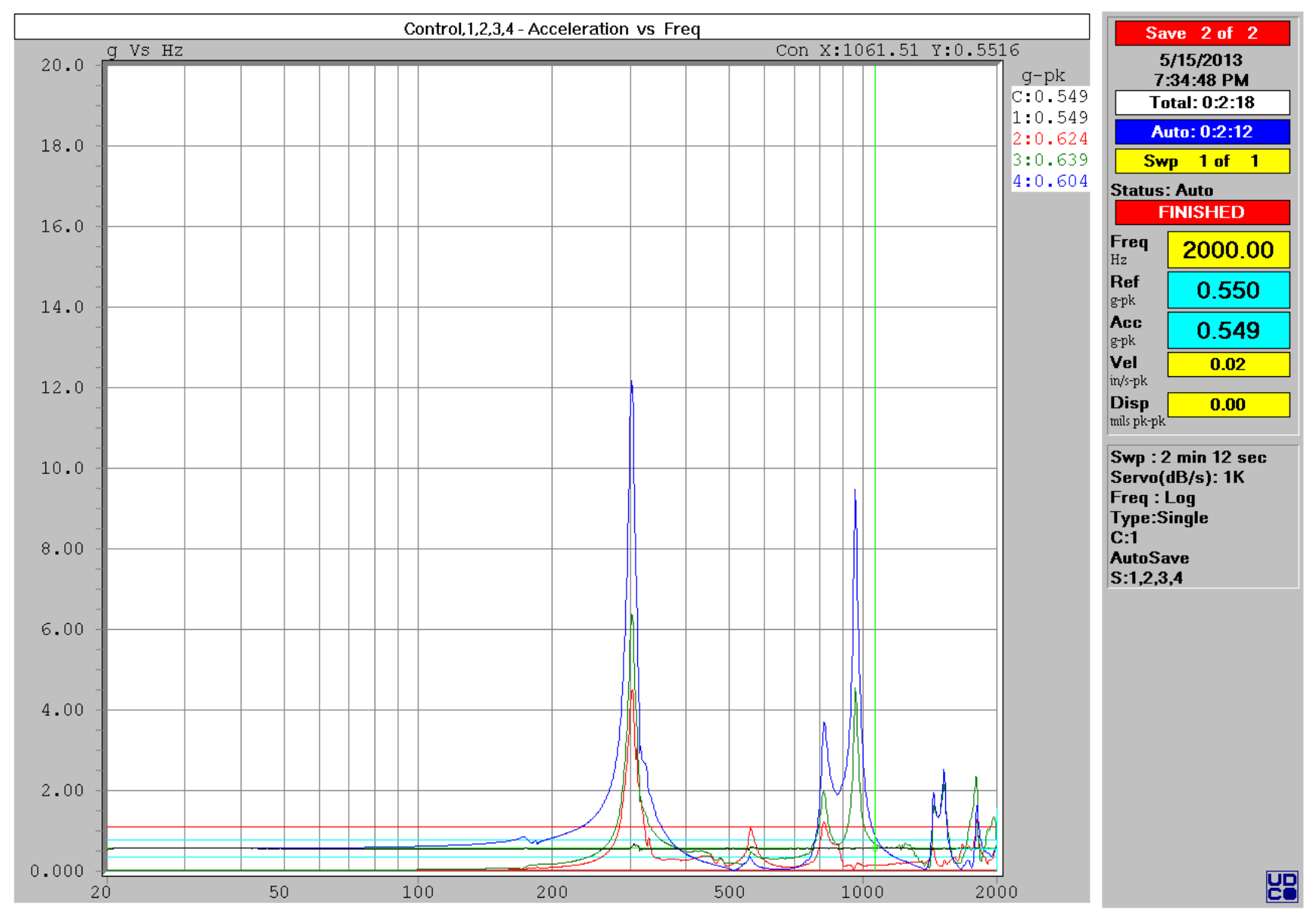

Figure 123: Raw data from test 13. 

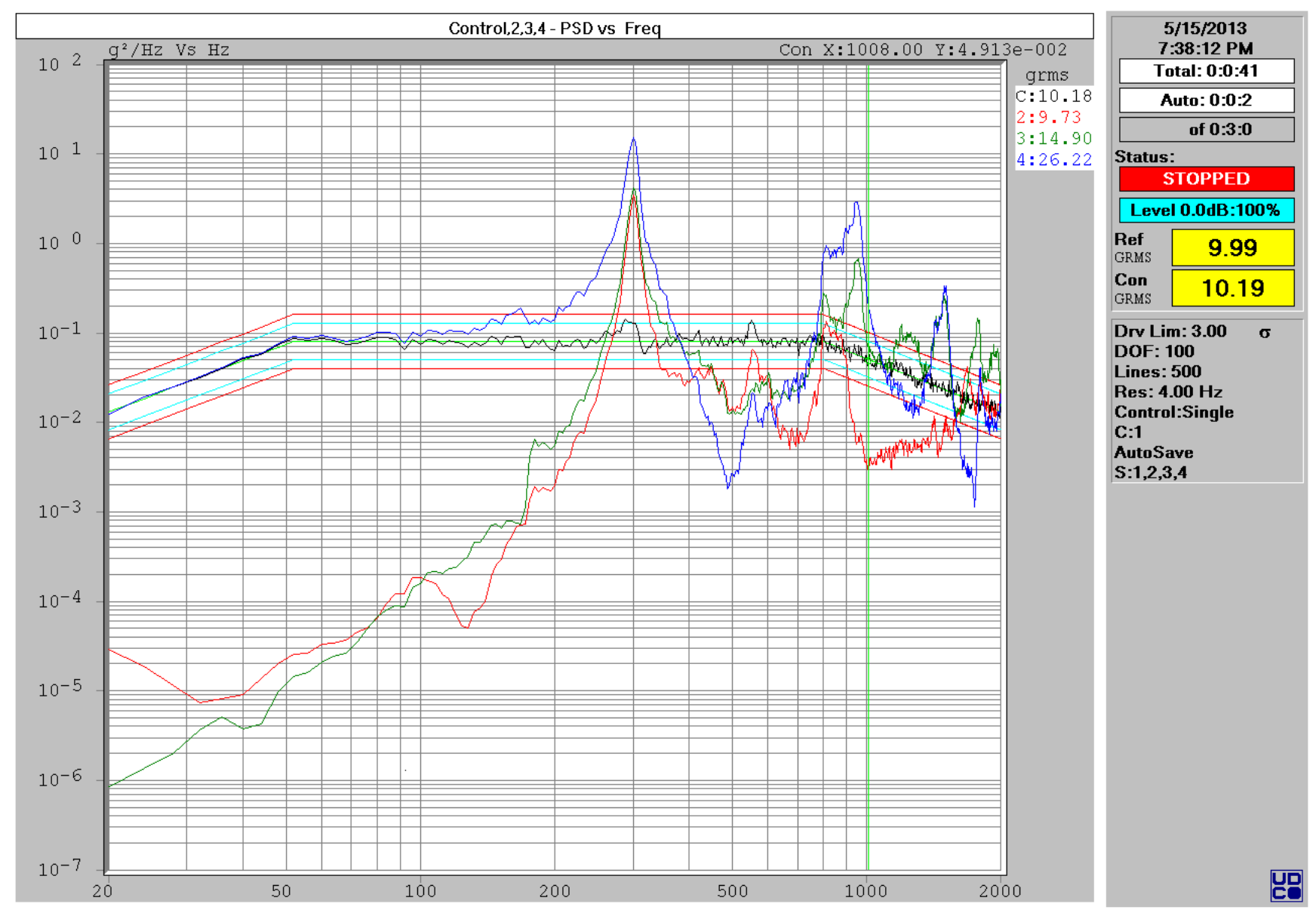

Figure 124: Raw data from test 14. 


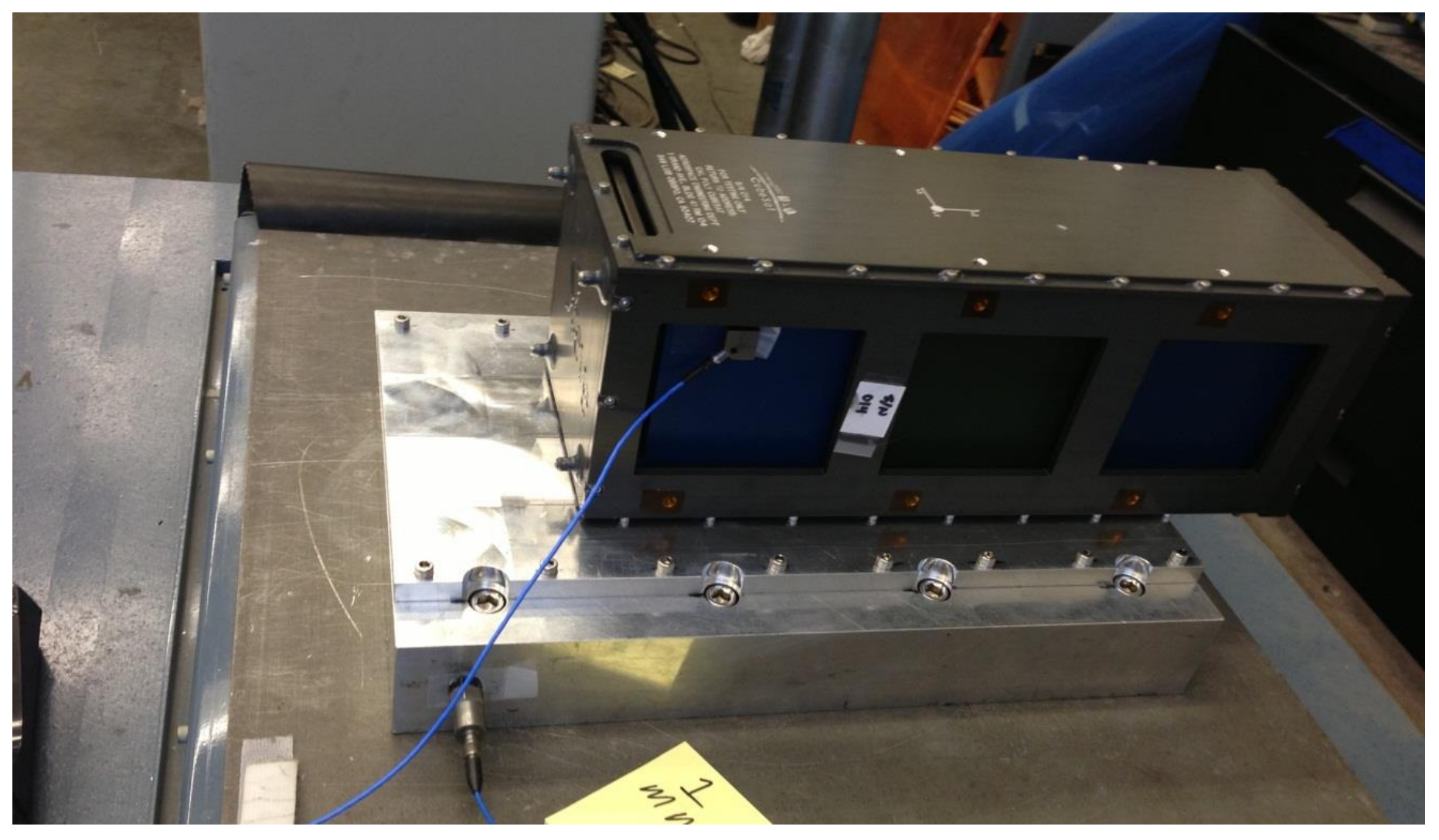

Figure 125: Setup for test 15-1. 


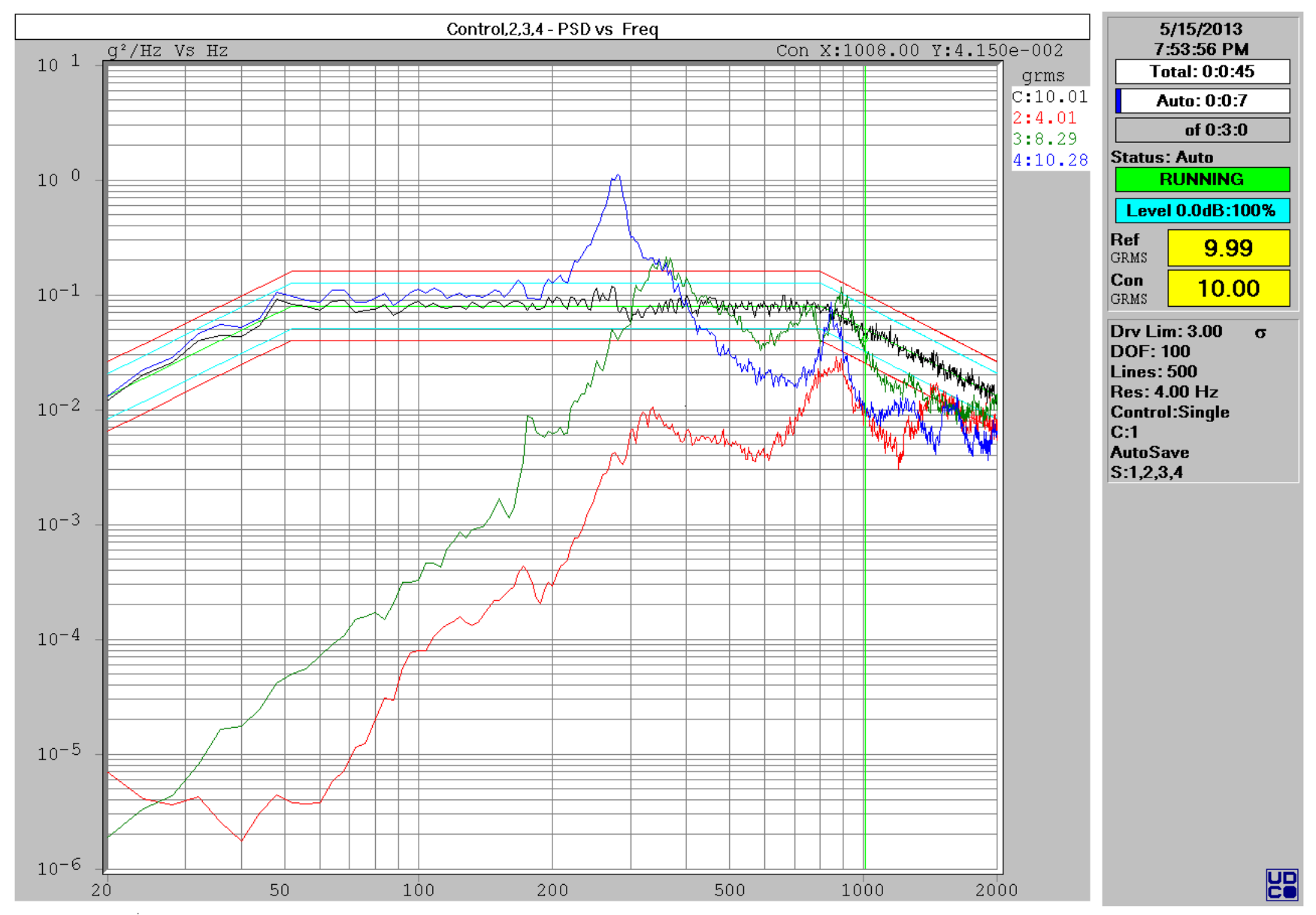

Figure 126: Raw data from test 15-1. 


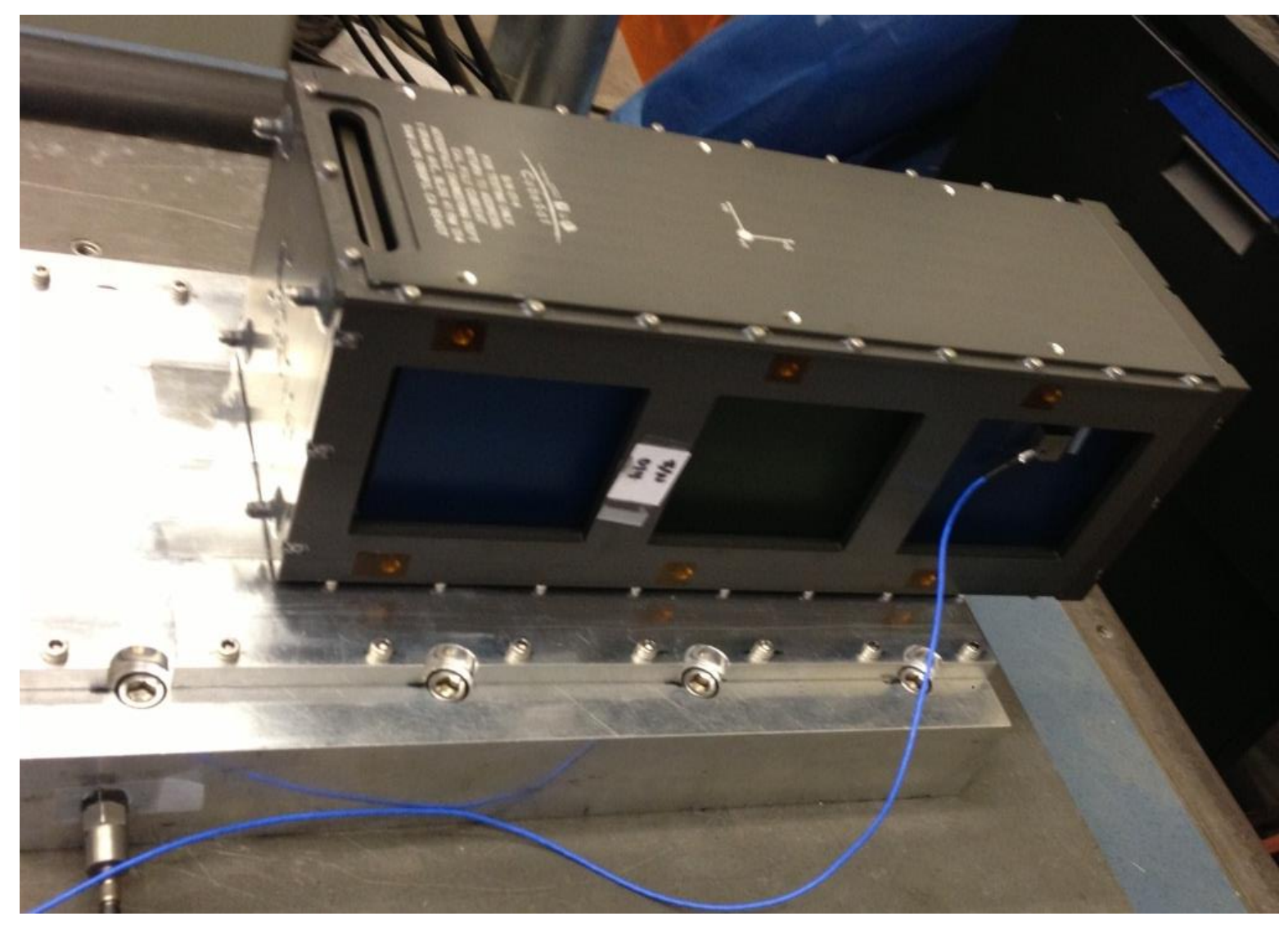

Figure 127: Setup for test 15-2. 


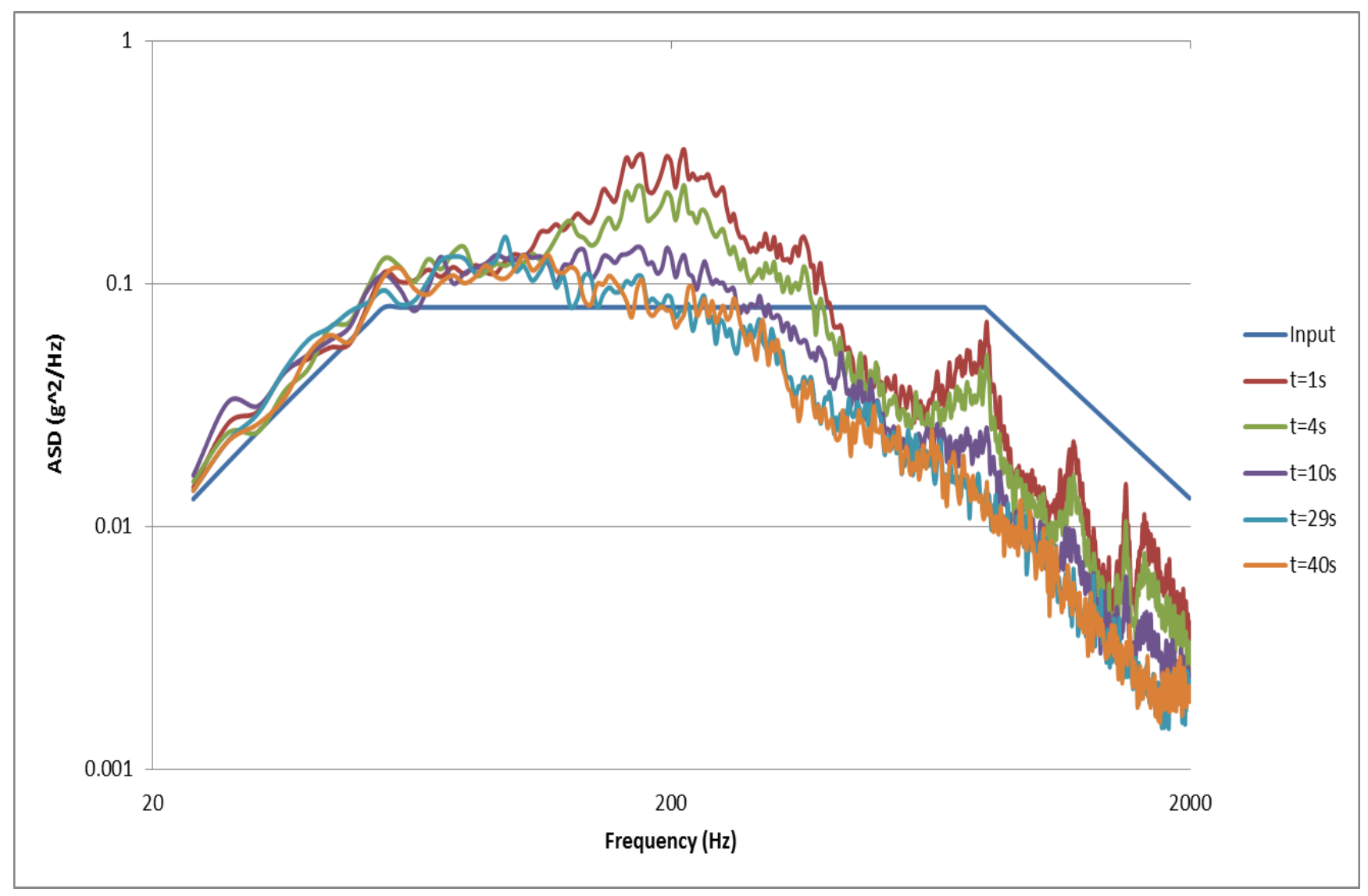

Figure 128: Time varying data from test number 15-2. 


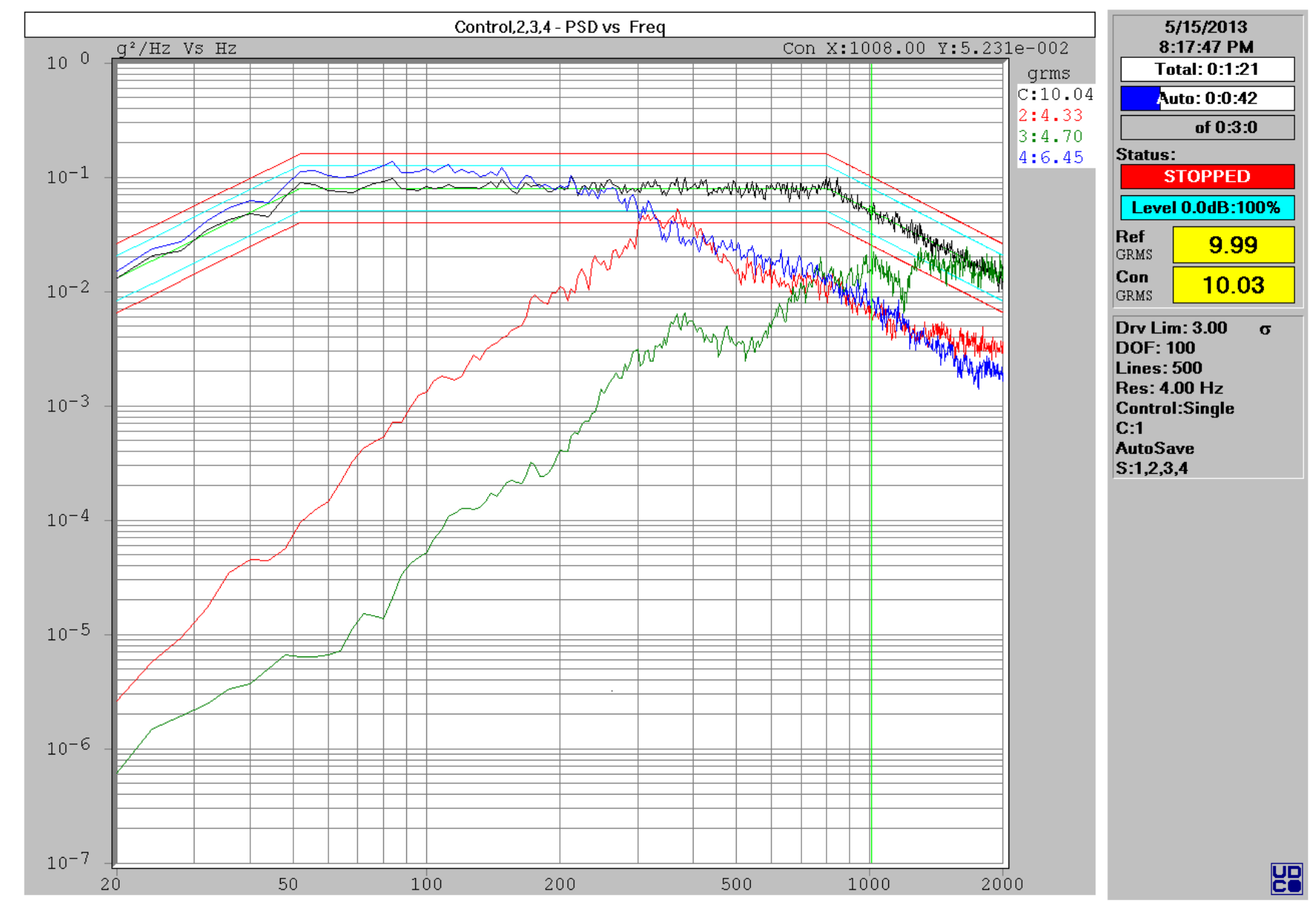

Figure 129: Raw data from test 15-2. 


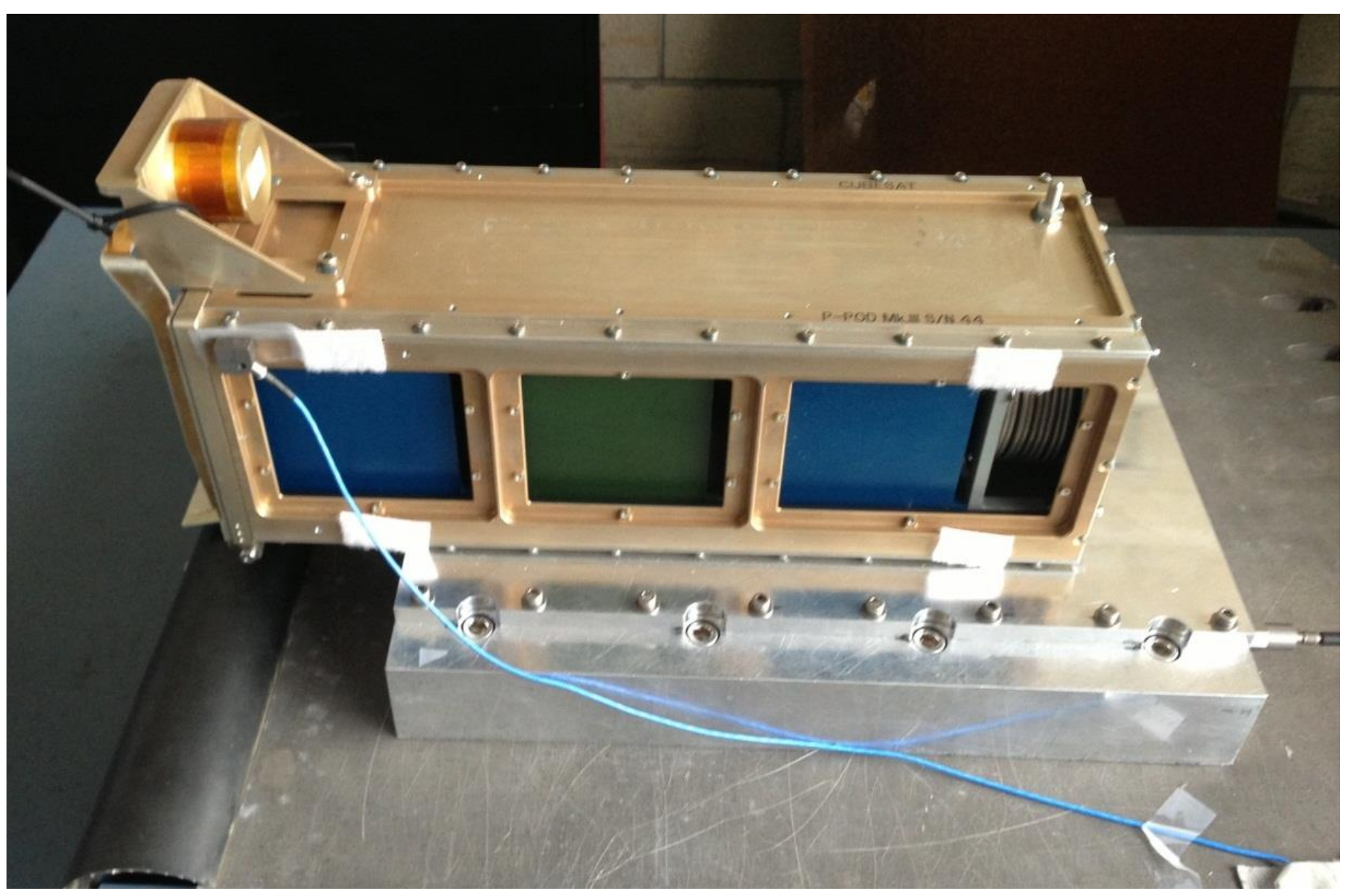

Figure 130: Setup for test numbers 16 and 17. 

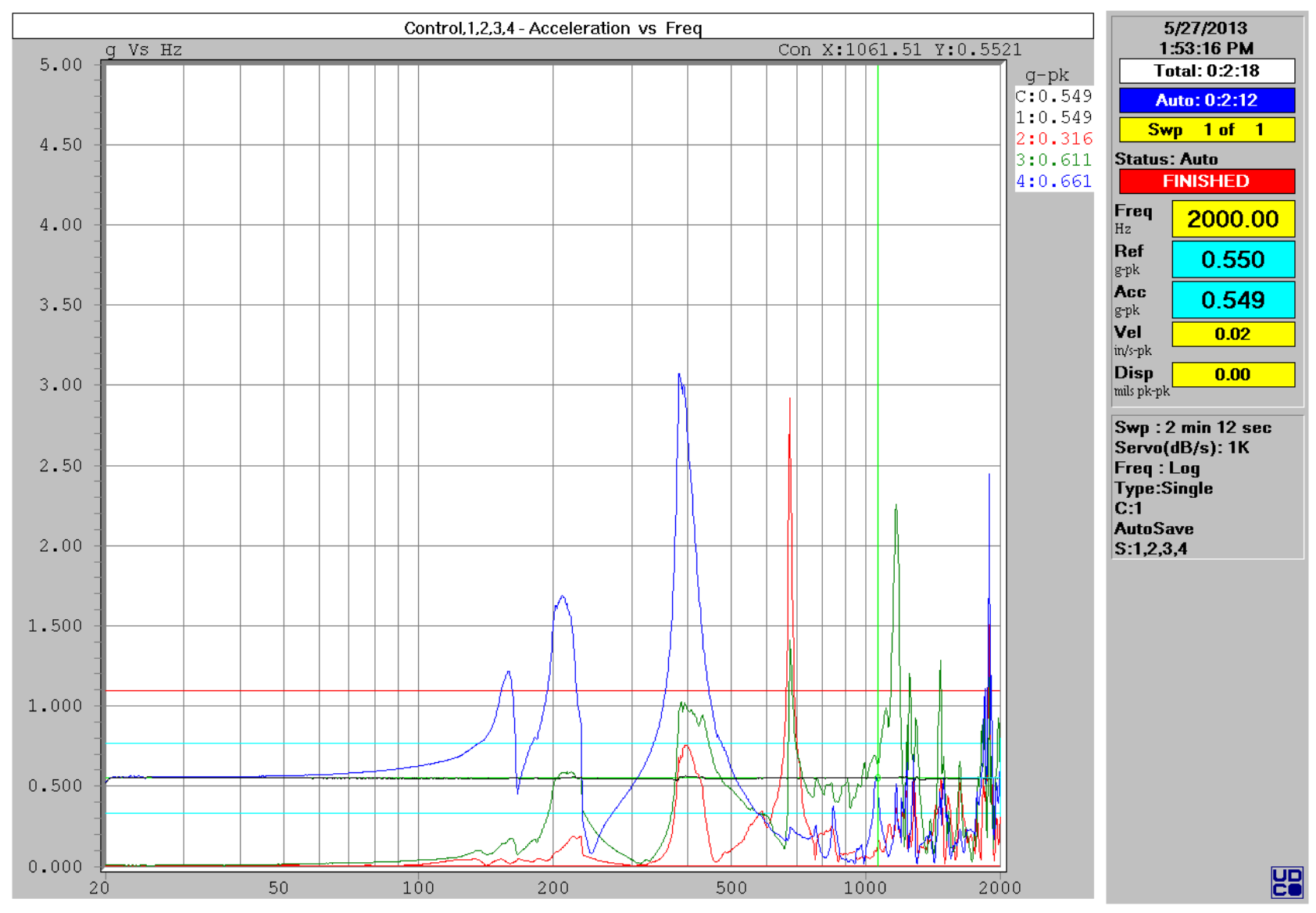

Figure 131: Raw data from test number 16. 


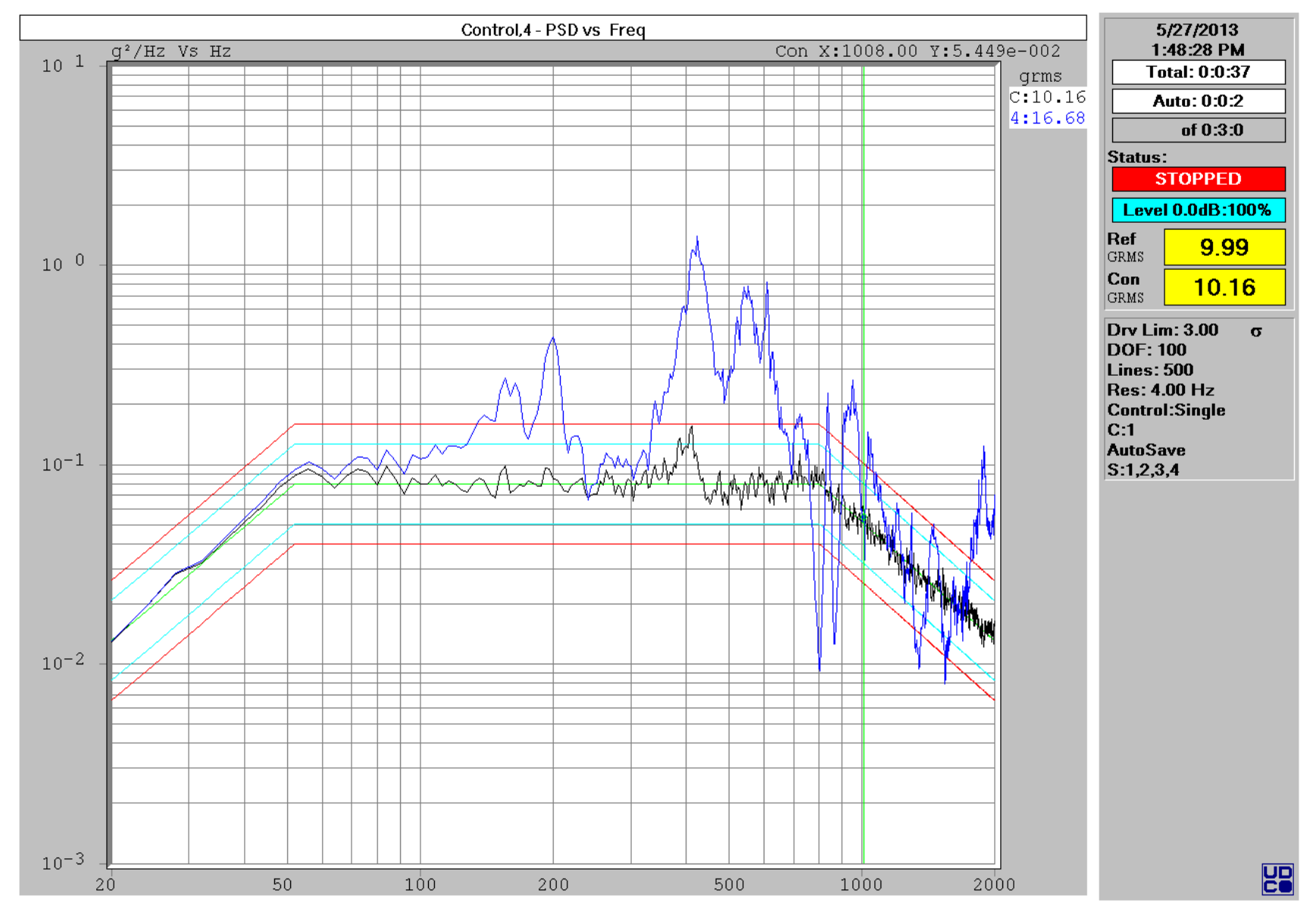

Figure 132: Raw data from test number 17. 


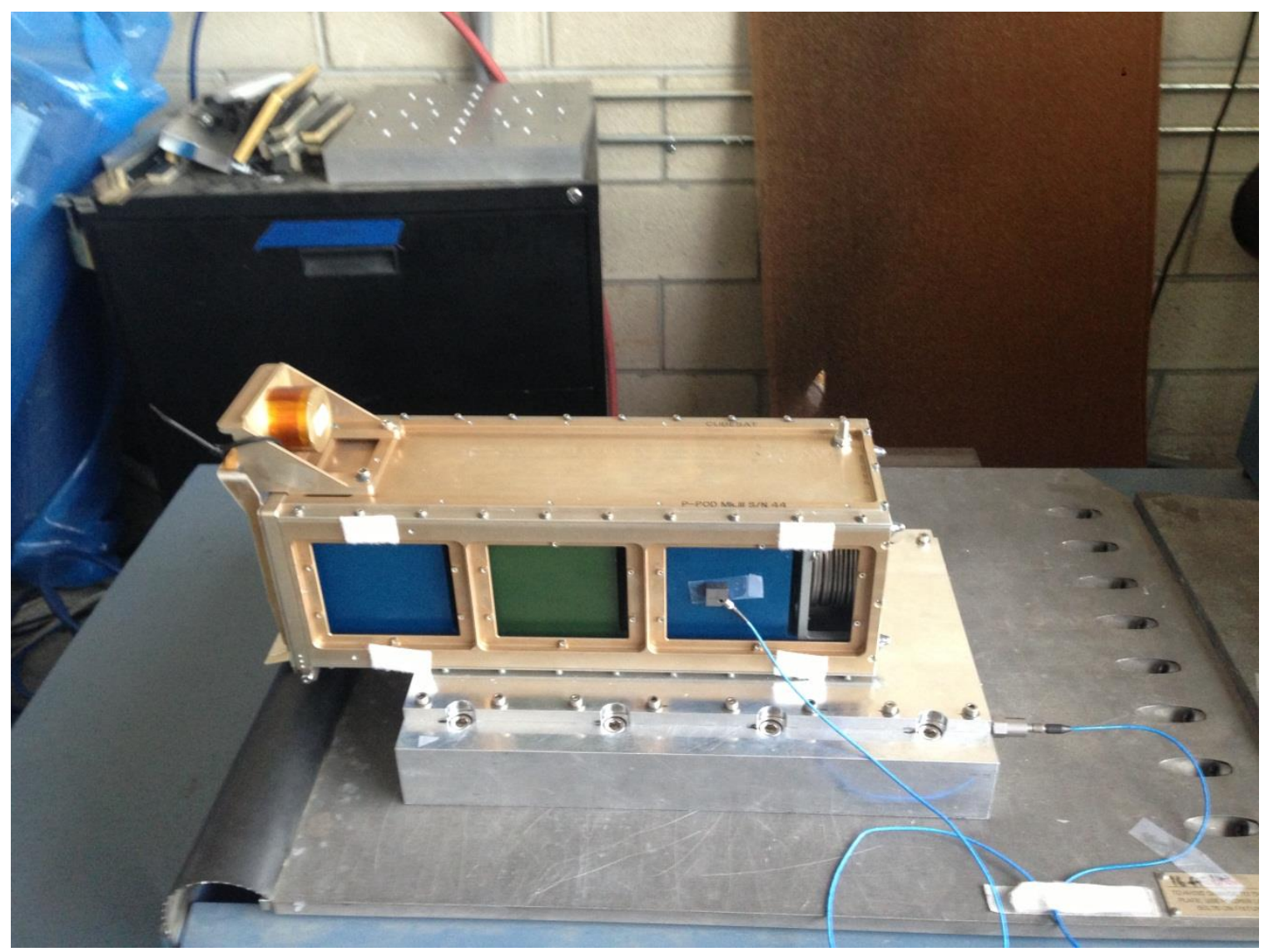

Figure 133: Setup for test number 18-1. 

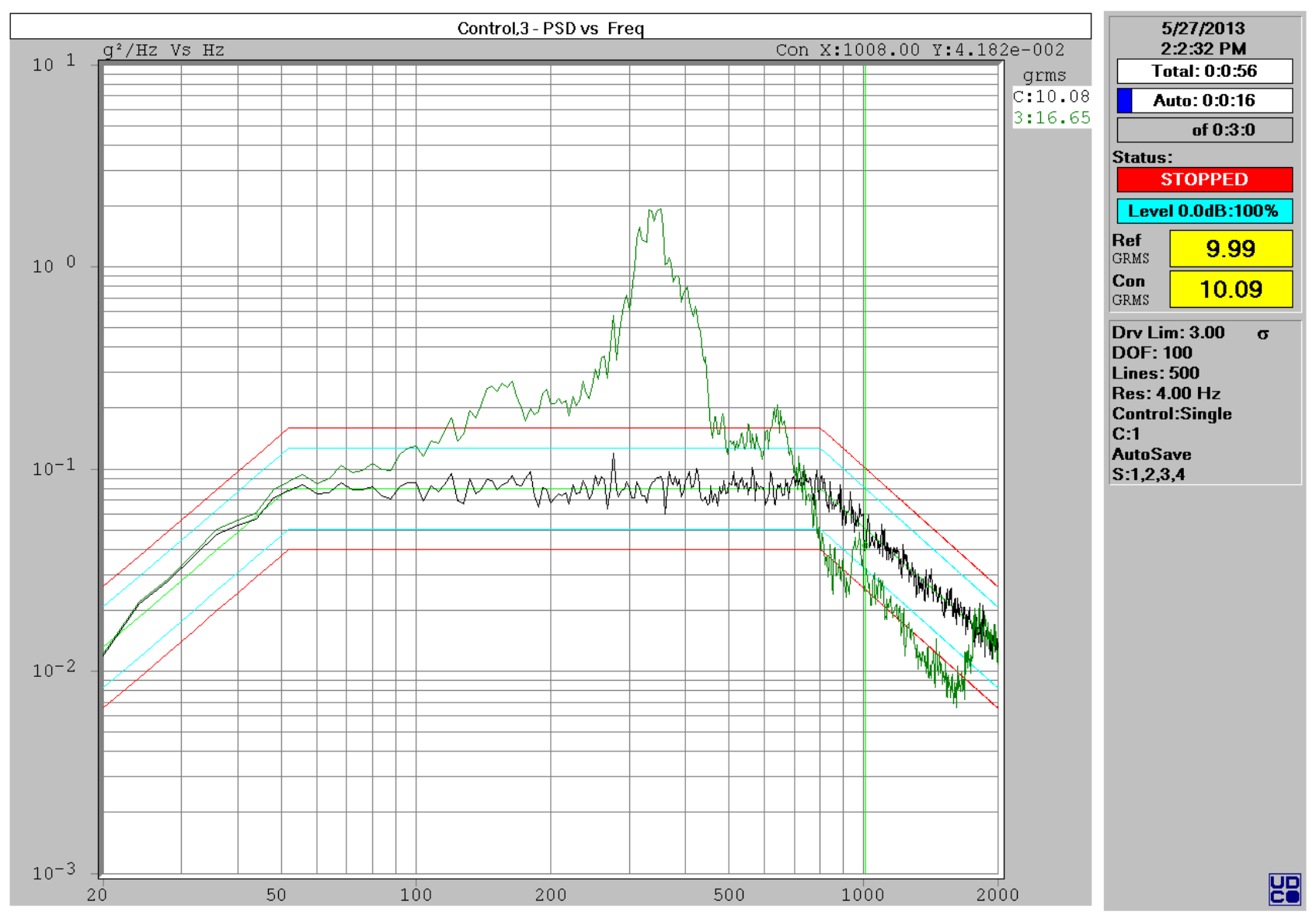

Figure 134: Raw data from test number 18-1. 


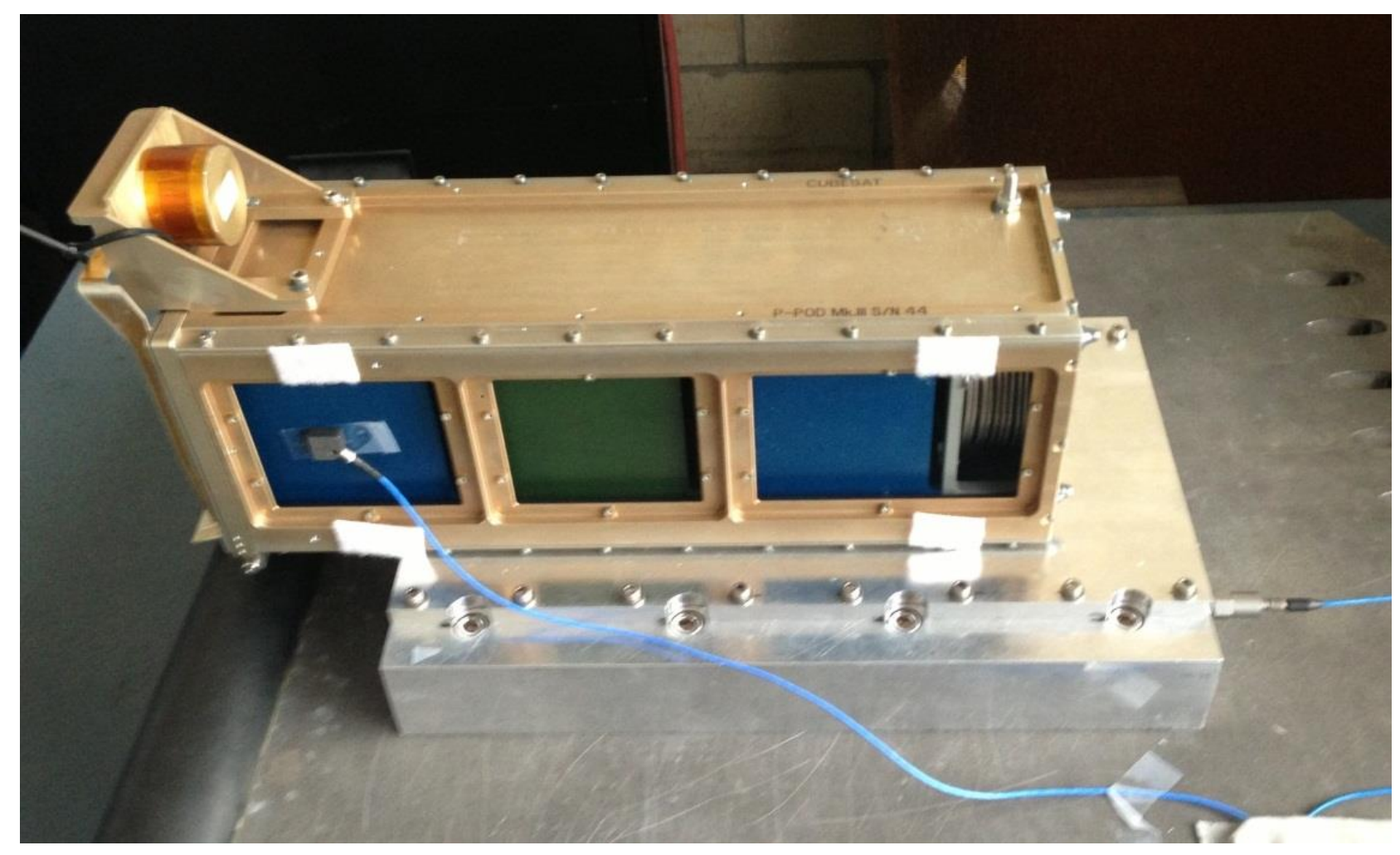

Figure 135: Setup for test numbers 18-2 and 19. 

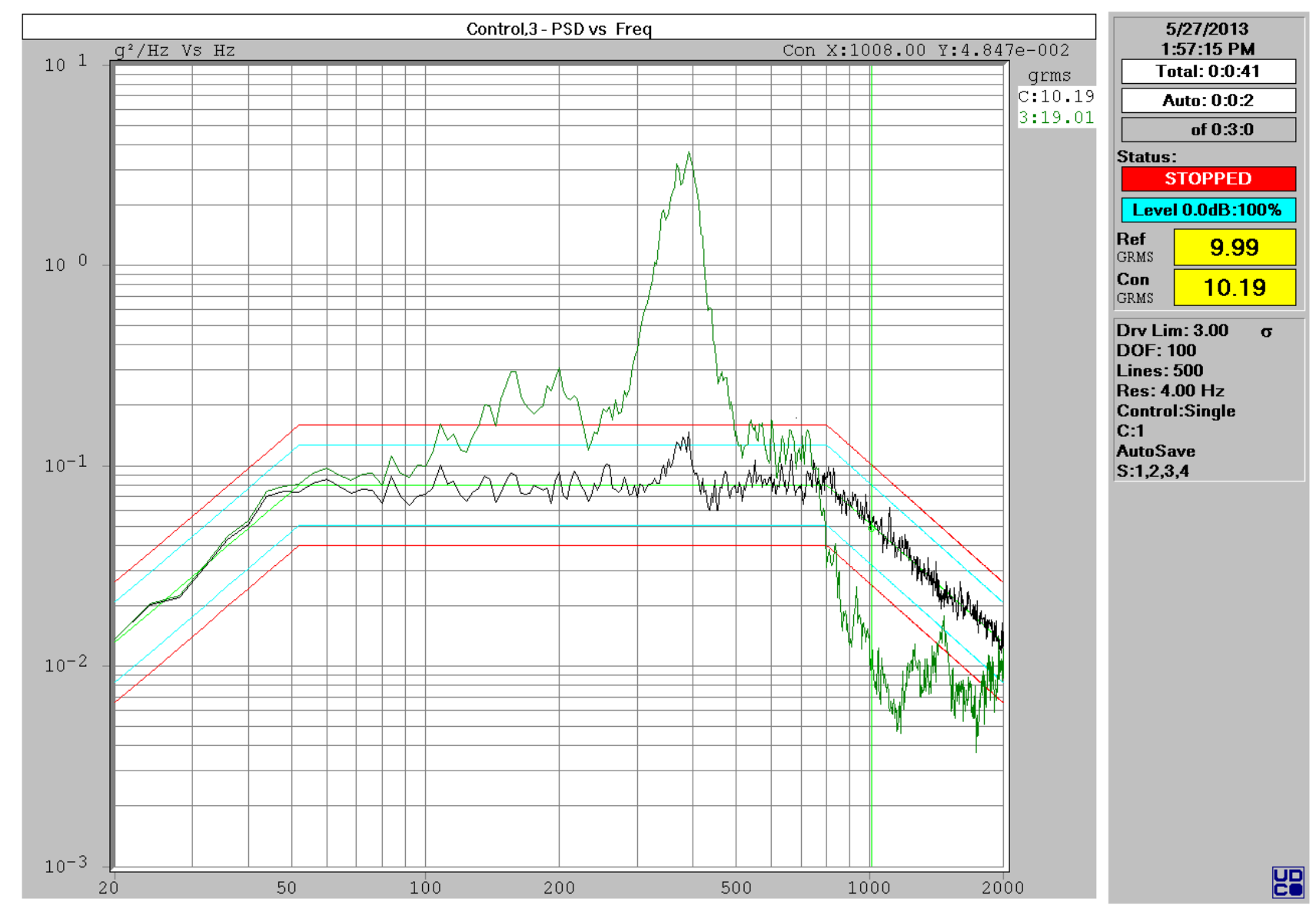

Figure 136: Raw data from test 18-2. 

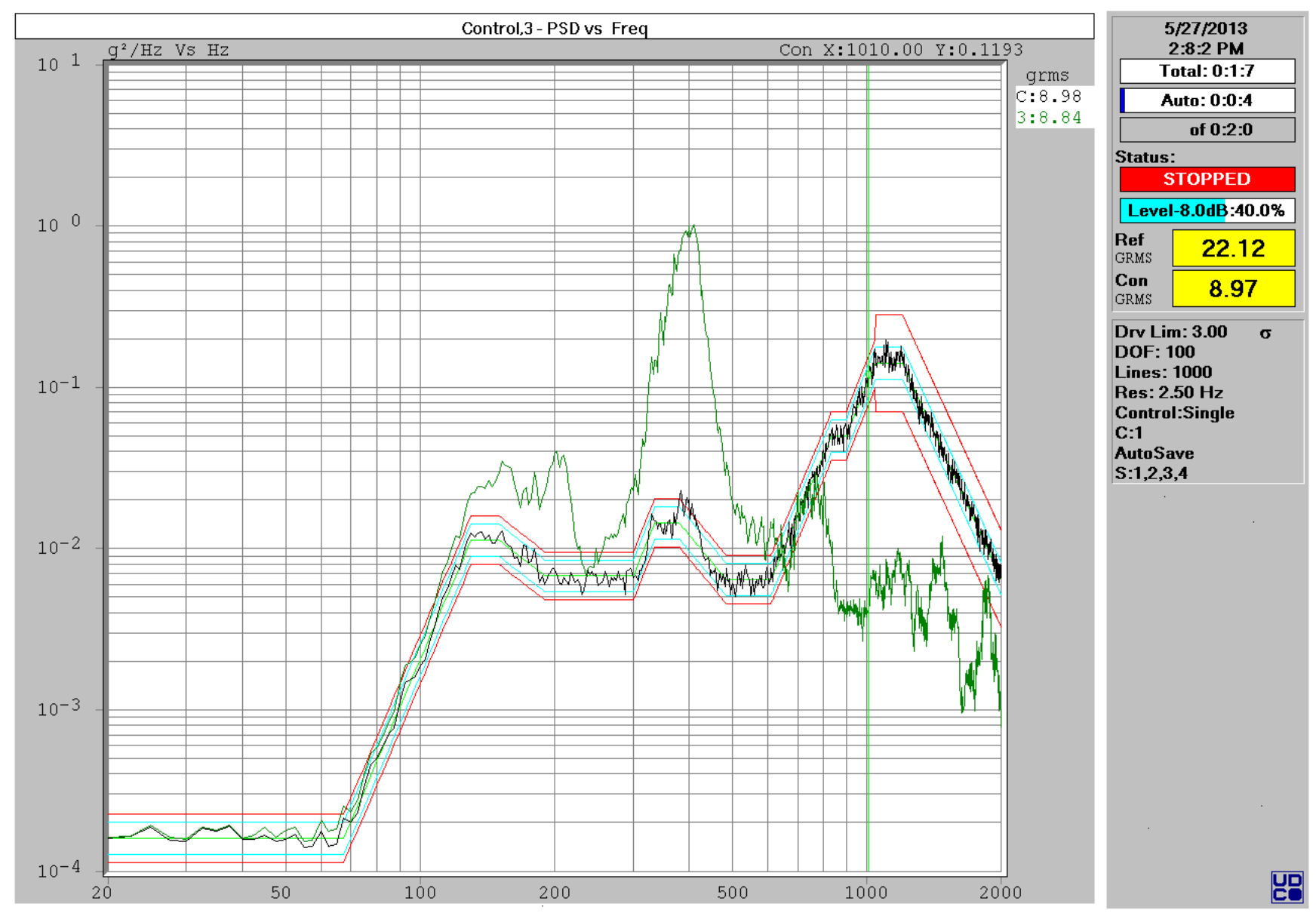

Figure 137: Raw data from test number 19. 


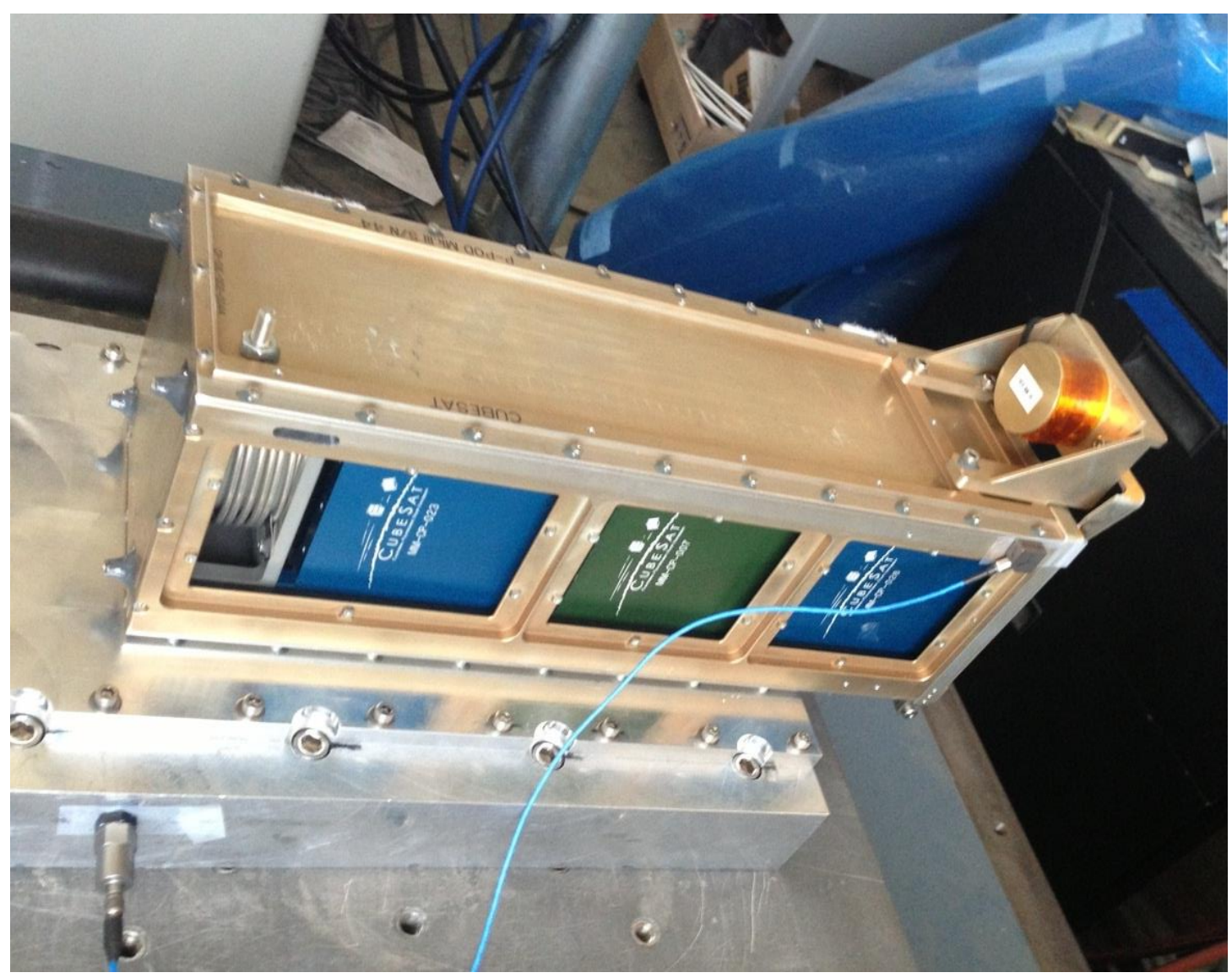

Figure 138: Setup for test number 20 and 21. 

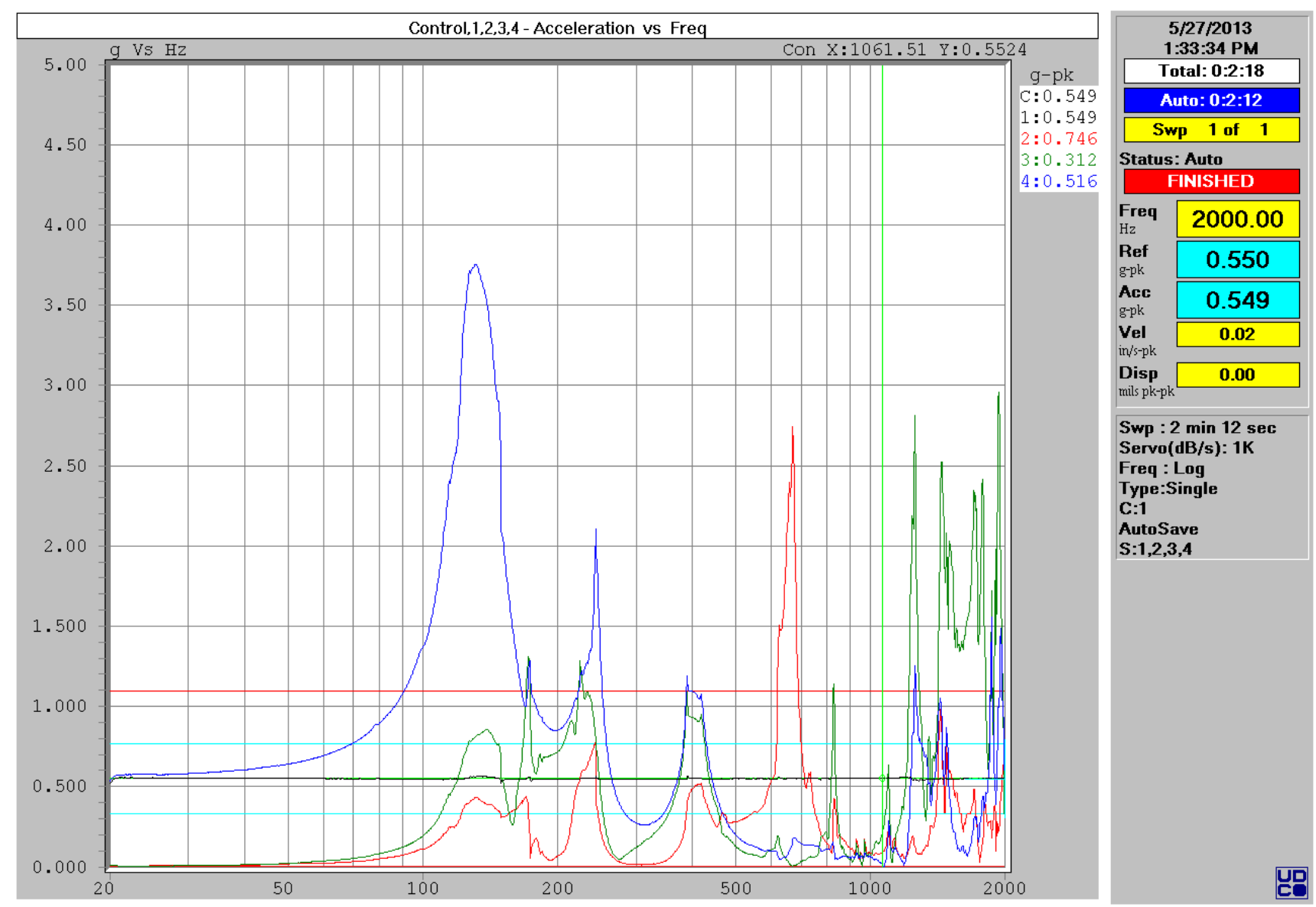

Figure 139: Raw data from test number 20. 

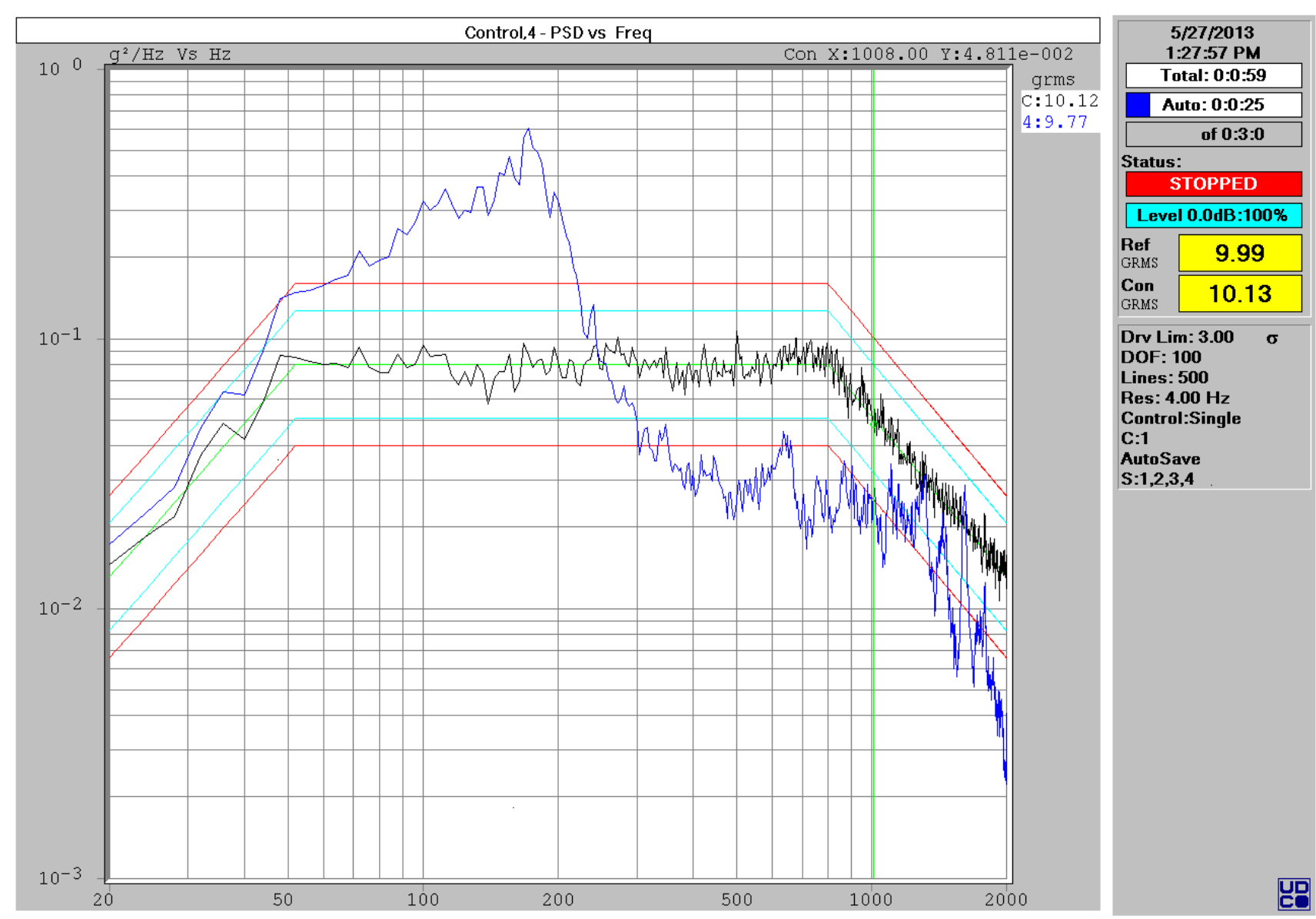

Figure 140: Raw data from test number 21. 


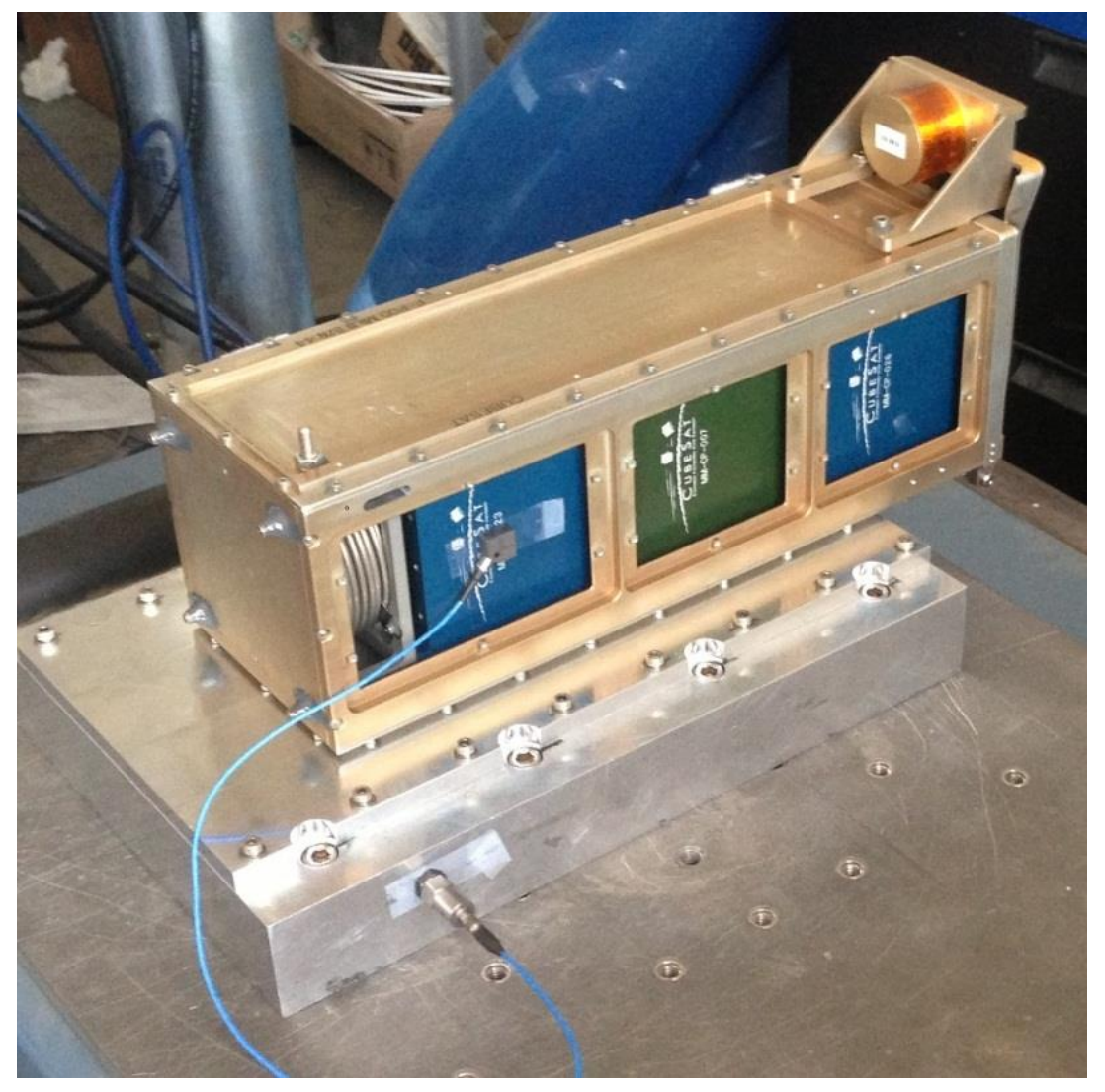

Figure 141: Setup from test number 22-1. 


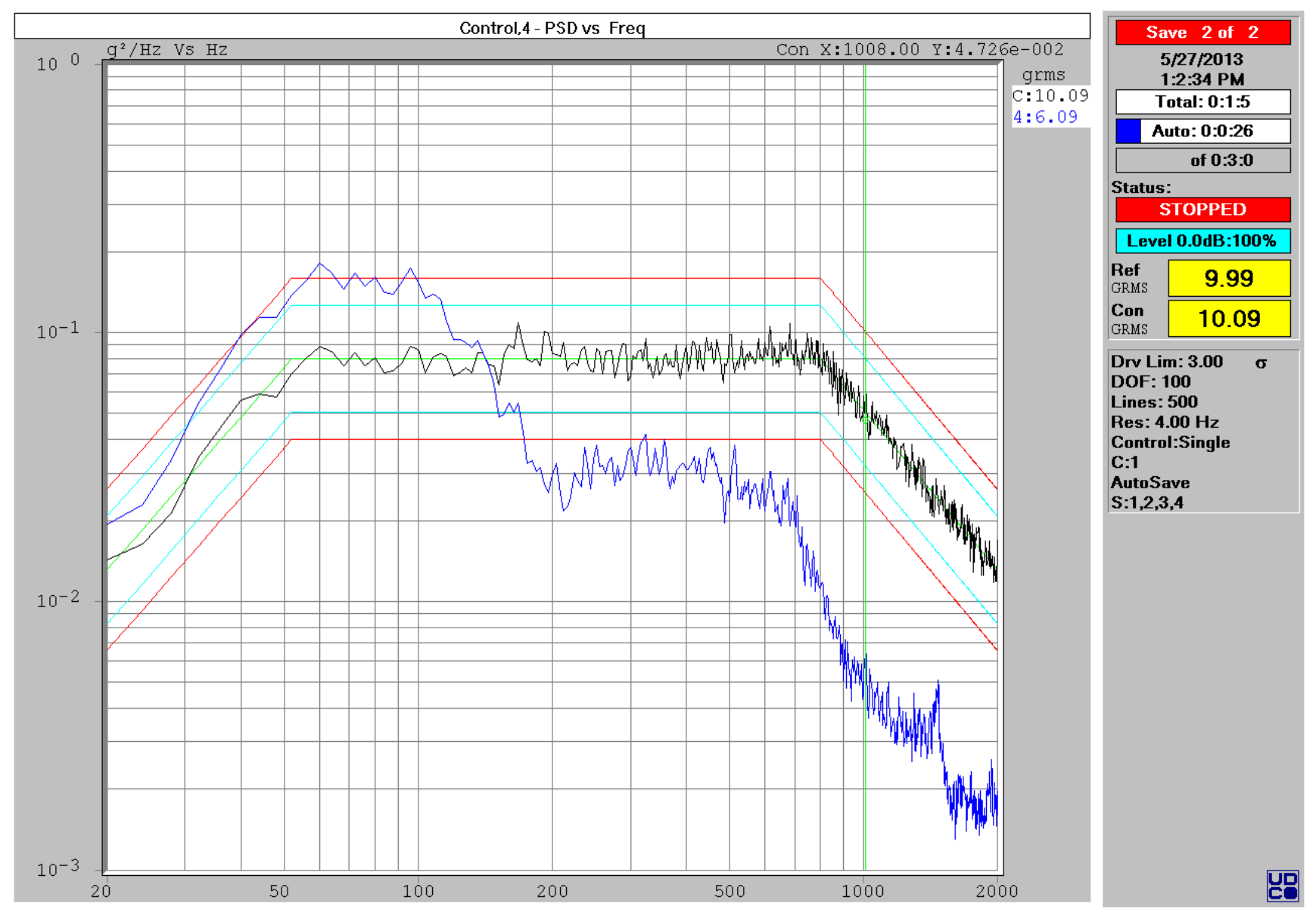

Figure 142: Raw data from test number 22-1. 


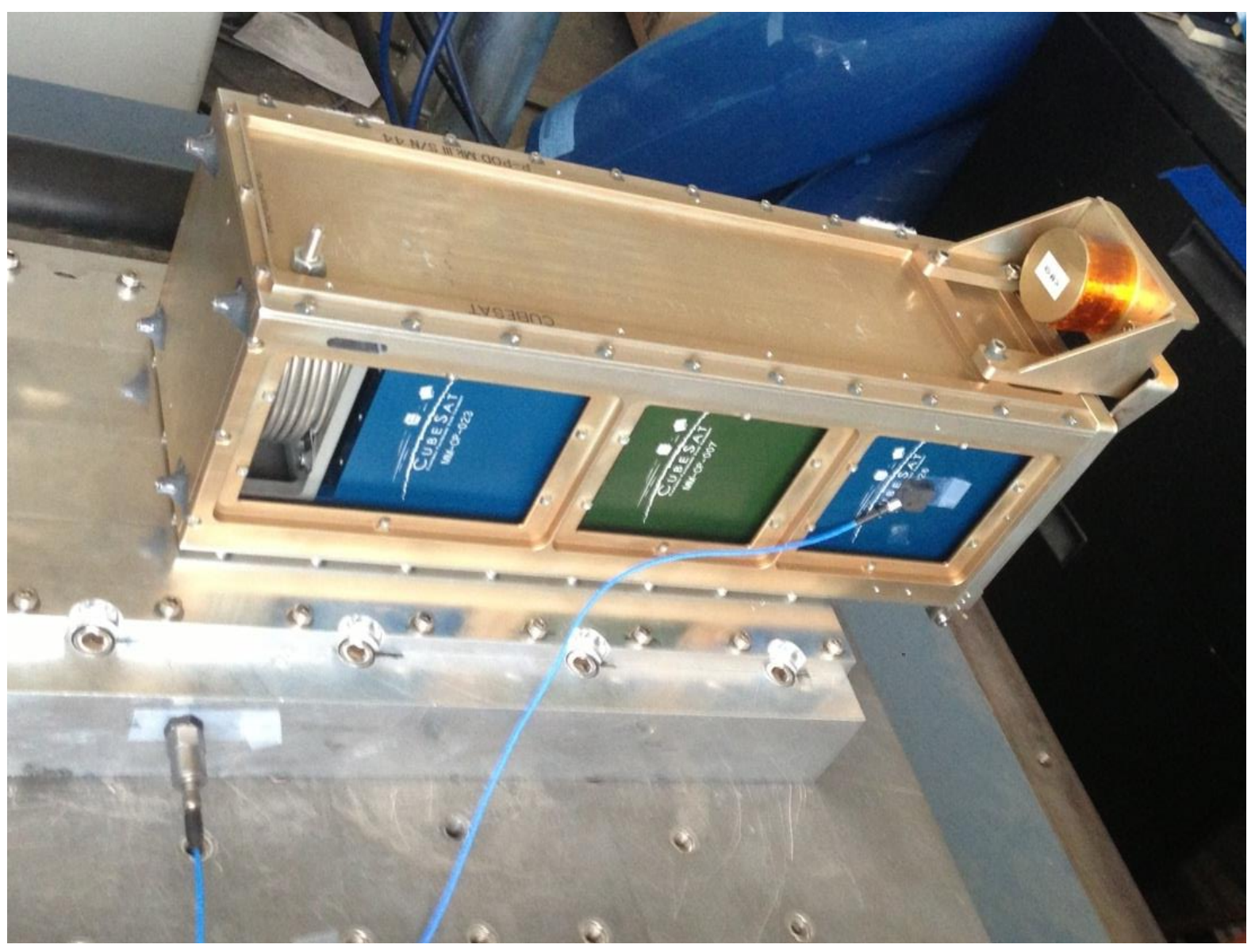

Figure 143: Setup for test numbers 22-2 and 23. 


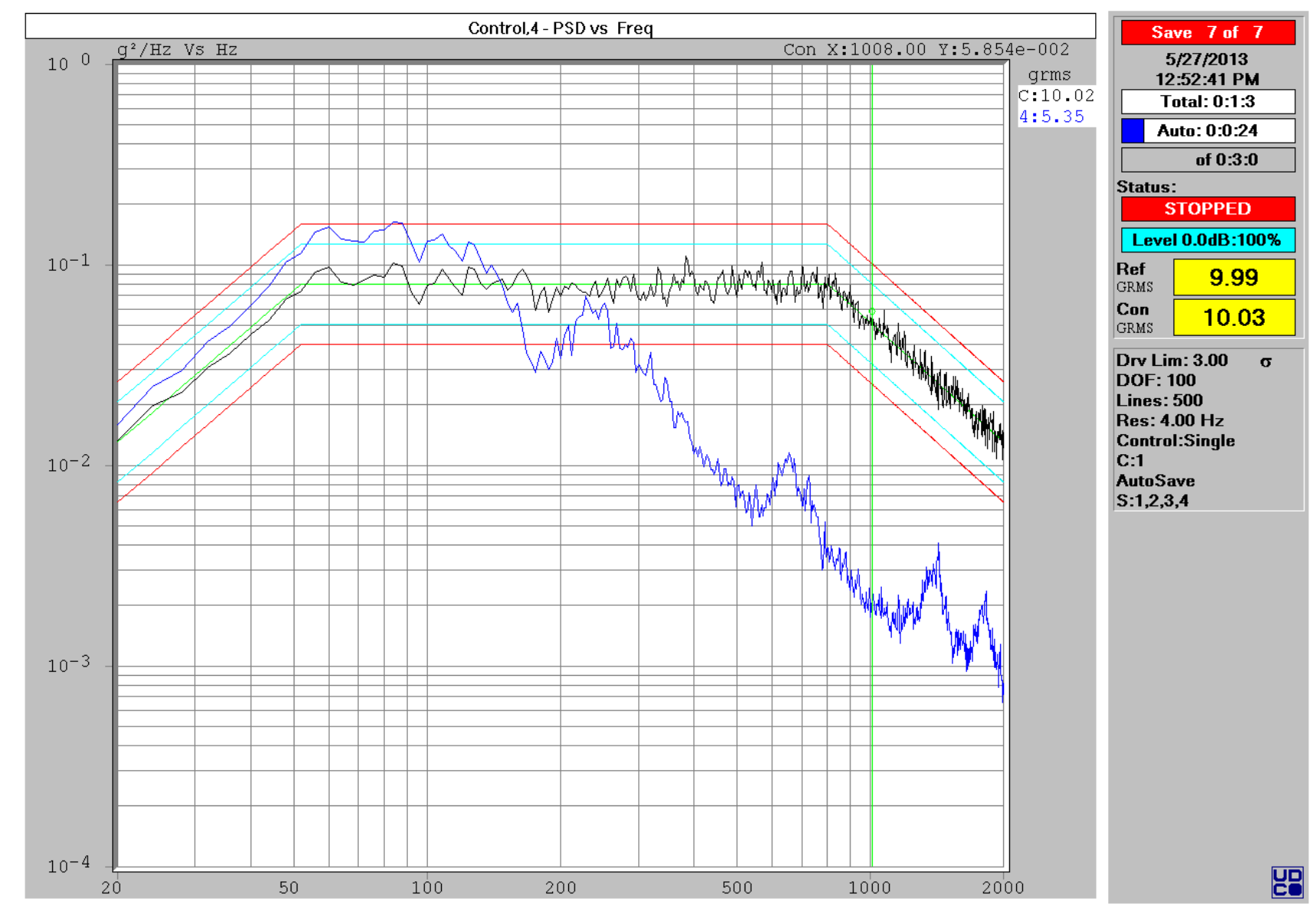

Figure 144: Raw data from test 22-2. 


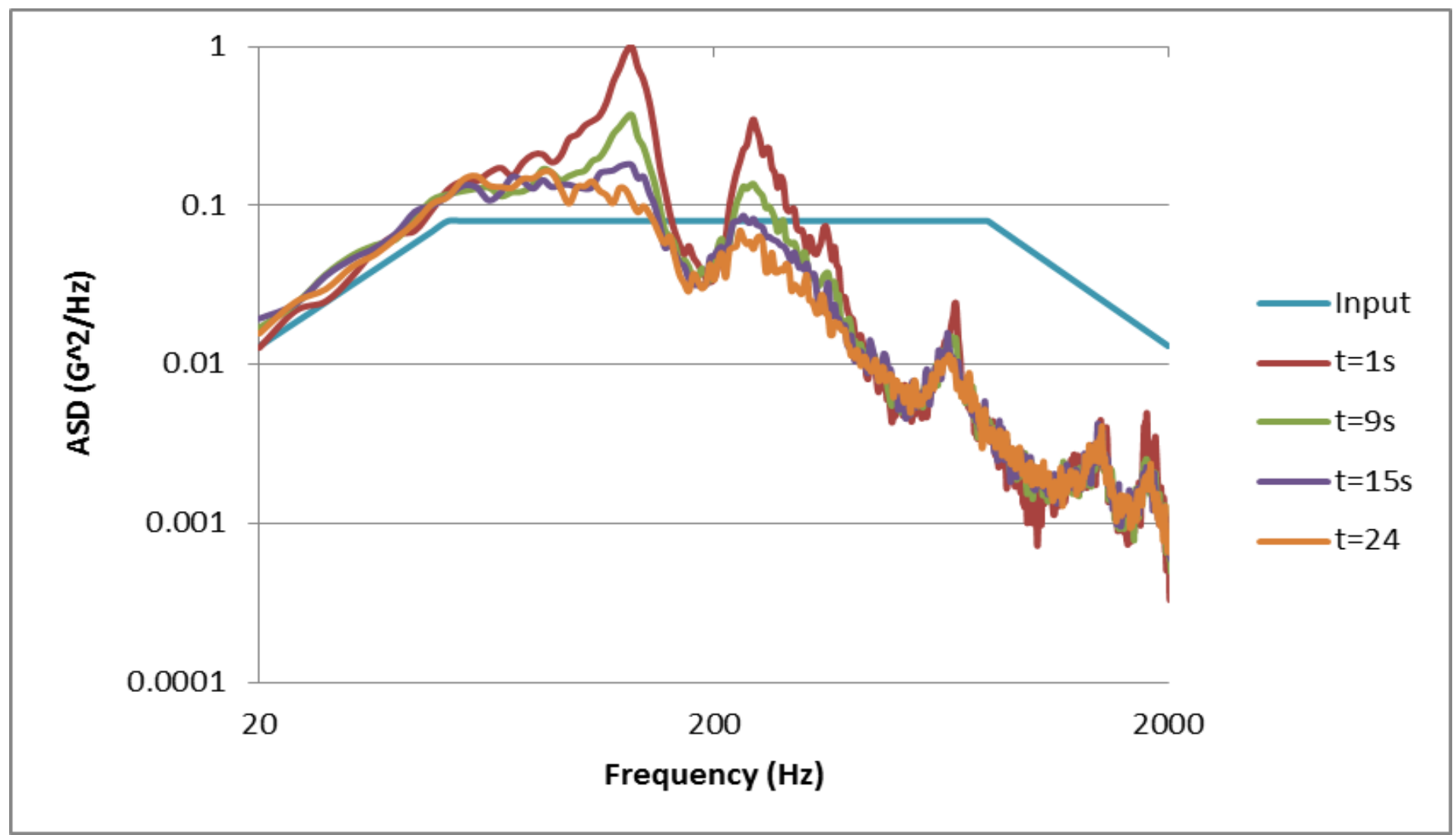

Figure 145: Time varying data from test number 22-2. 

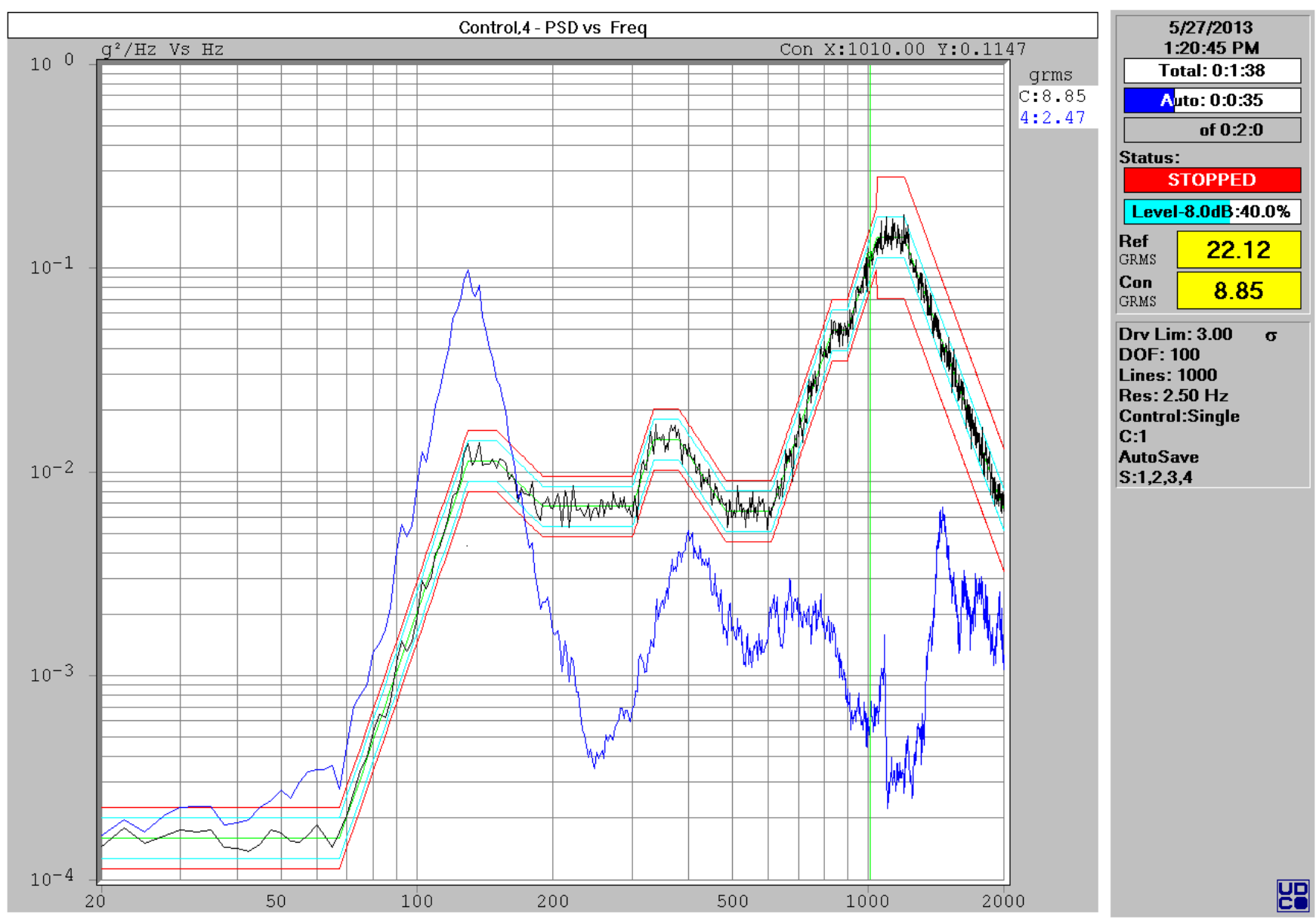

Figure 146: Raw data from test number 23. 


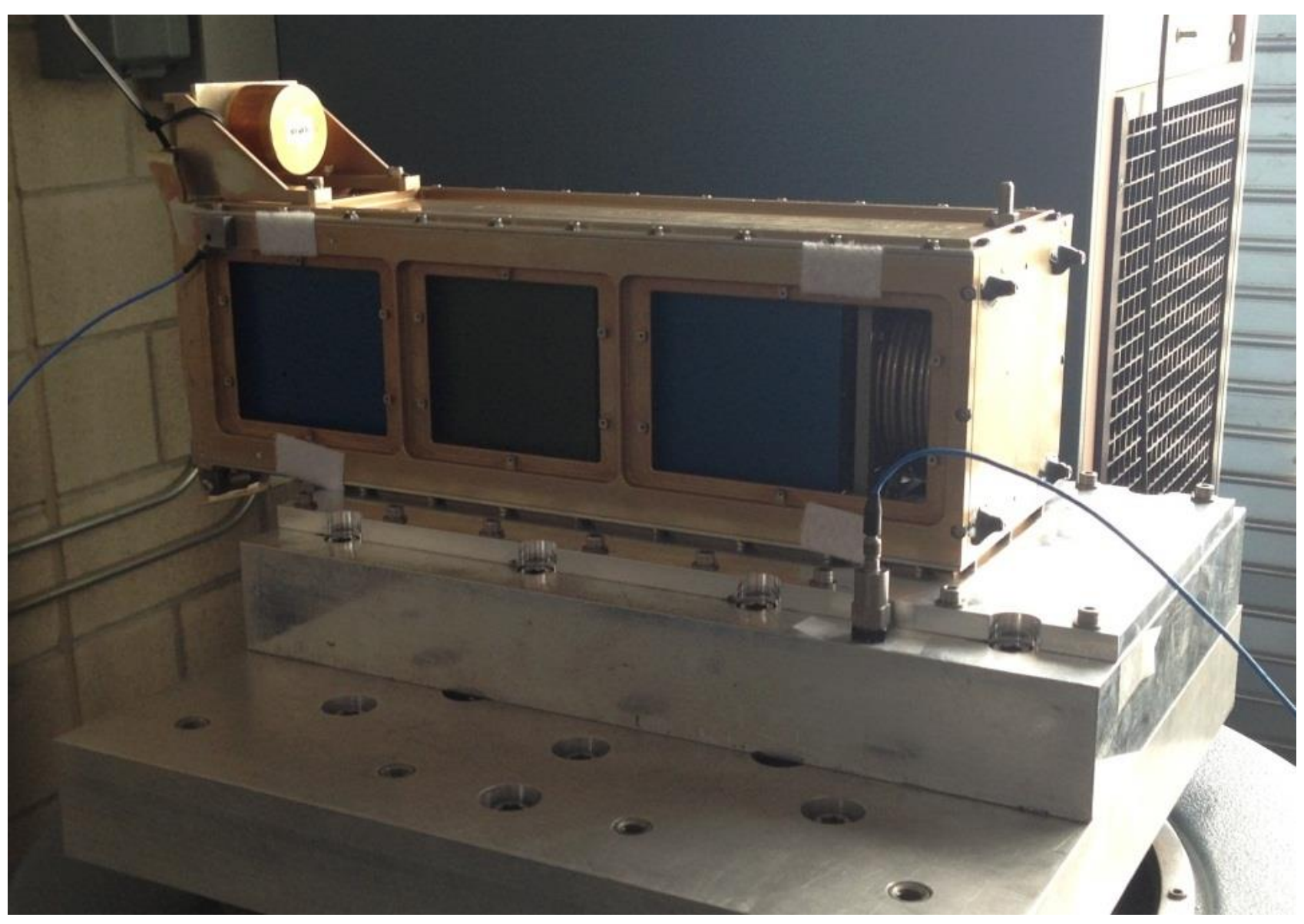

Figure 147: Setup for test numbers 24 and 25. 

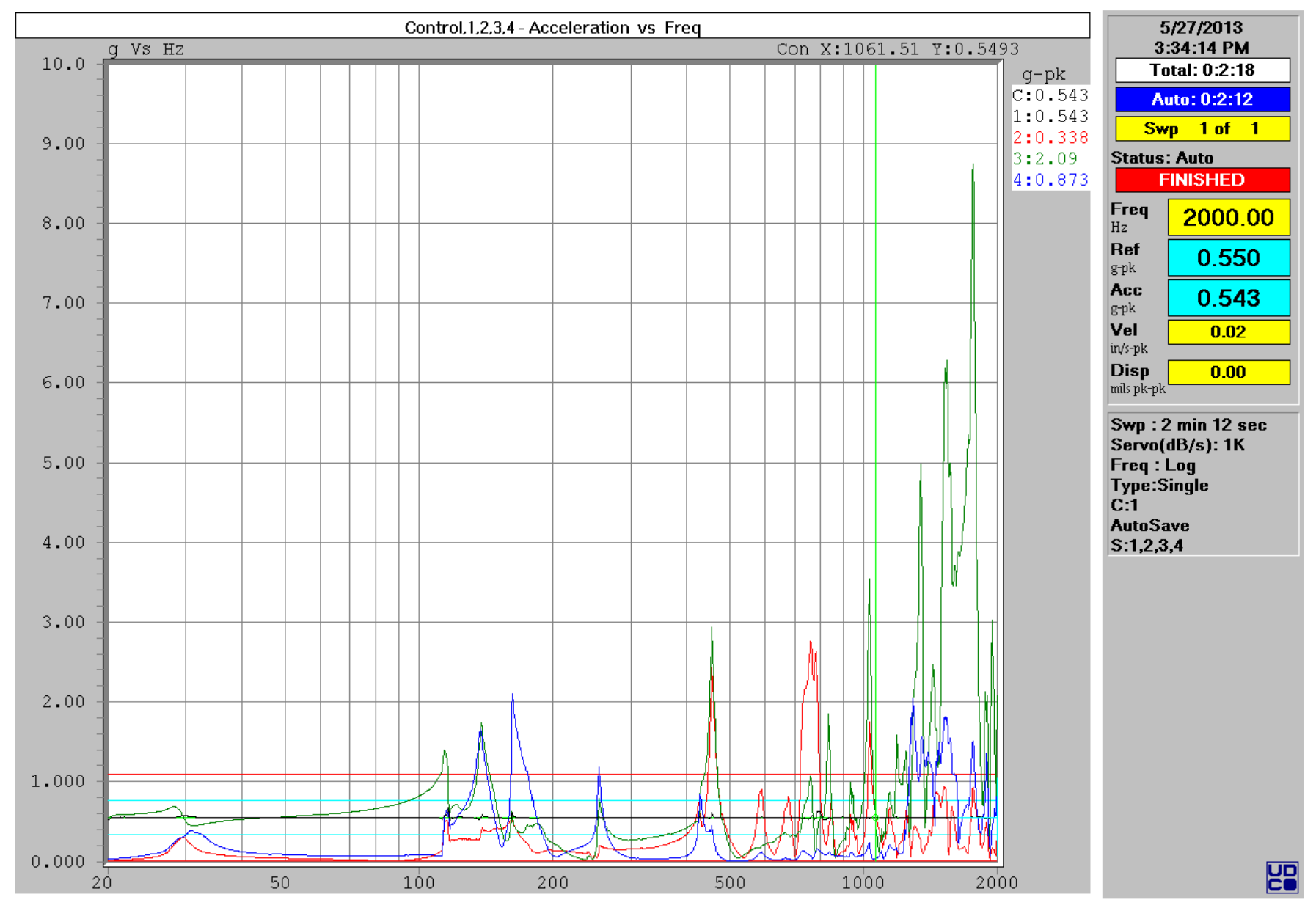

Figure 148: Raw data from test number 24. 


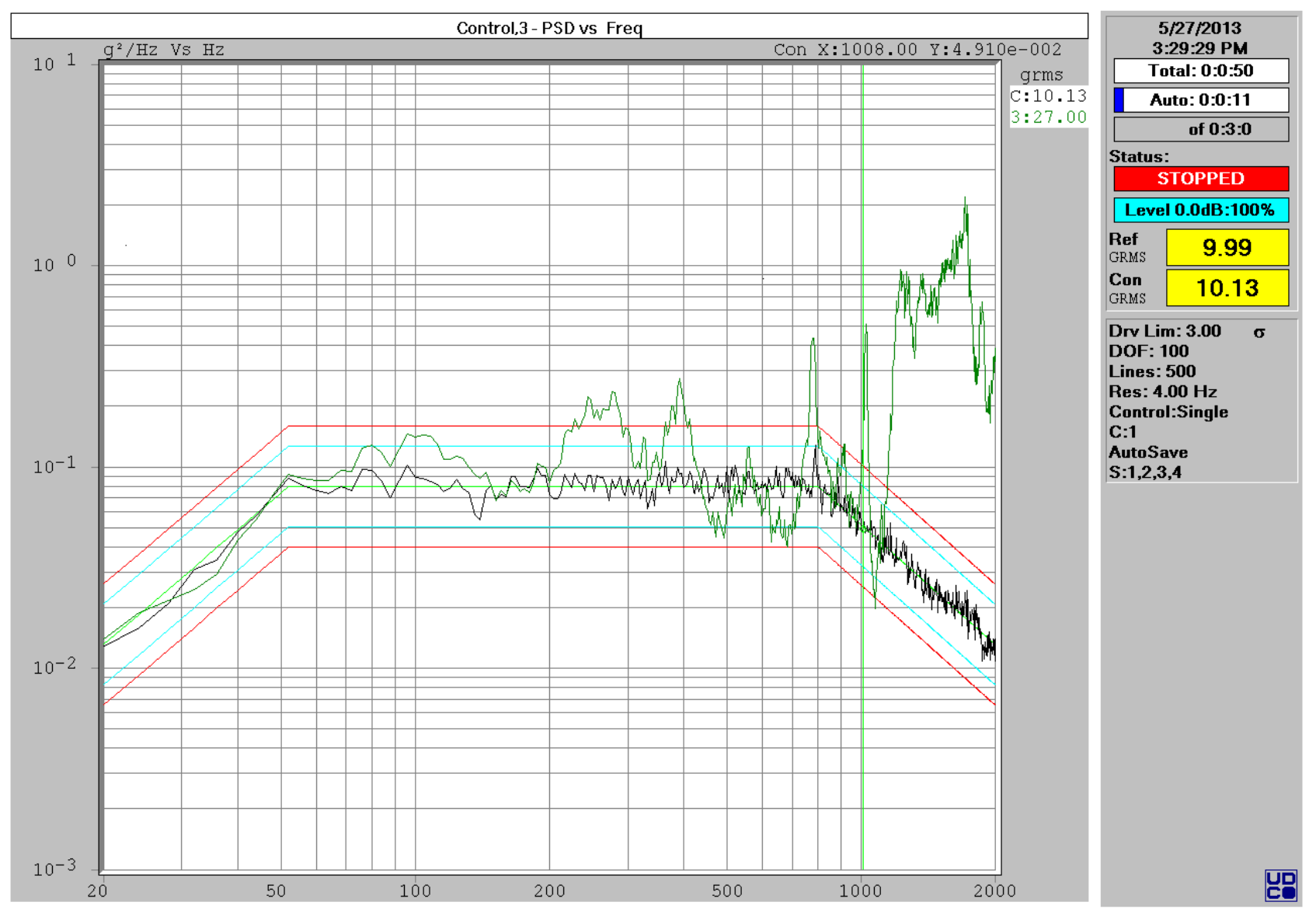

Figure 149: Raw data from test number 25. 


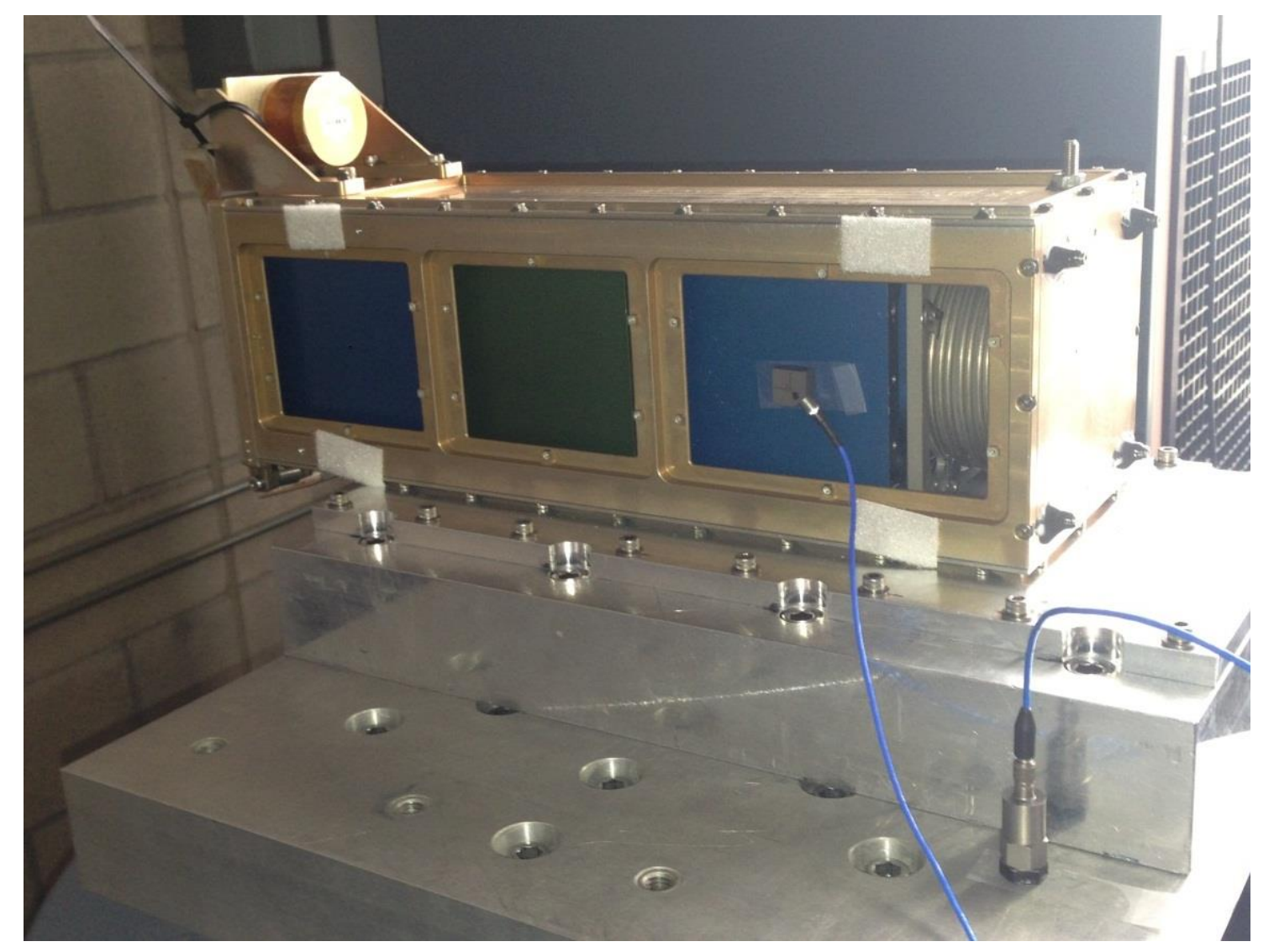

Figure 150: Setup for test number 26-1. 


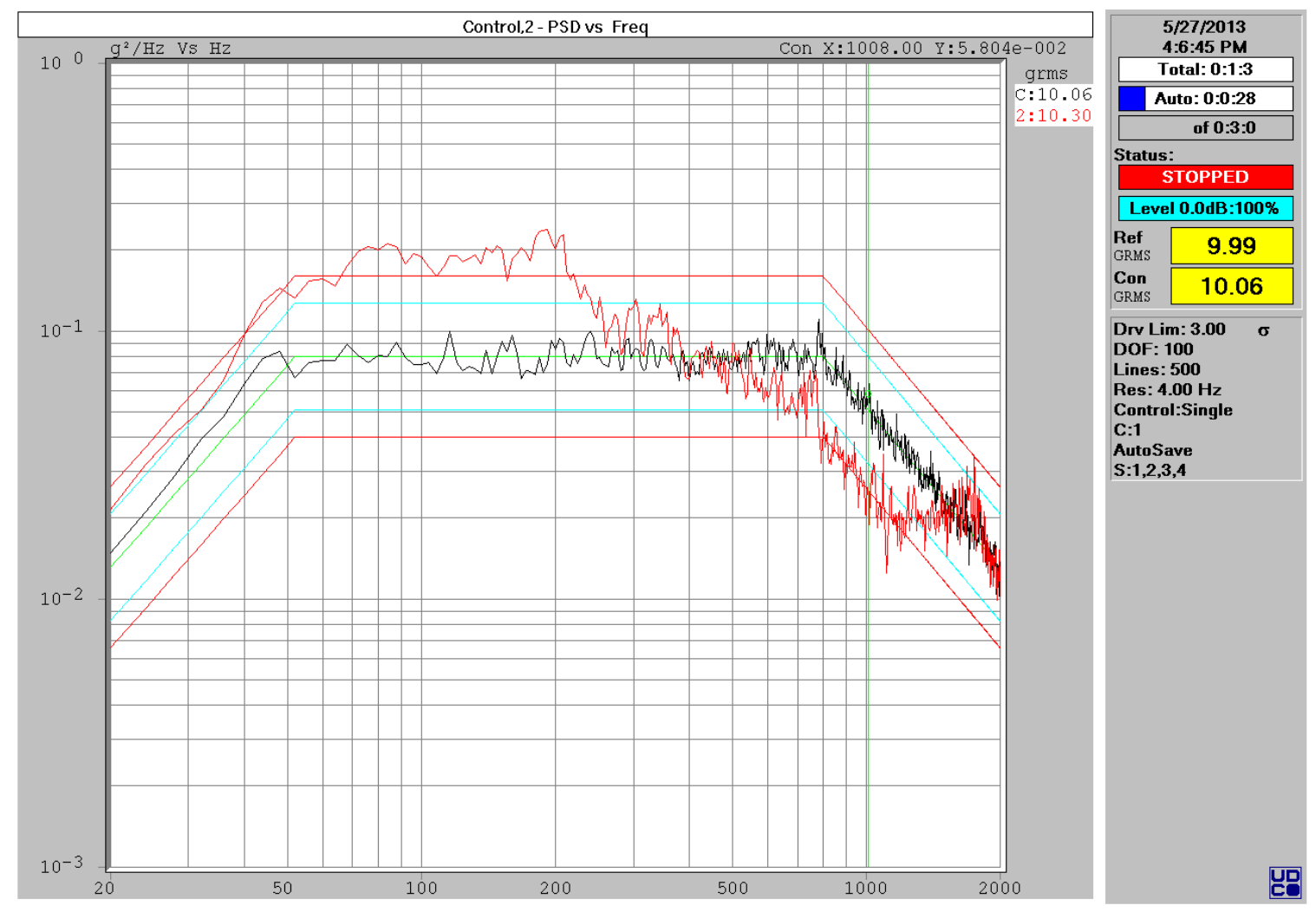

Figure 151: Raw data from test number 26-1. 


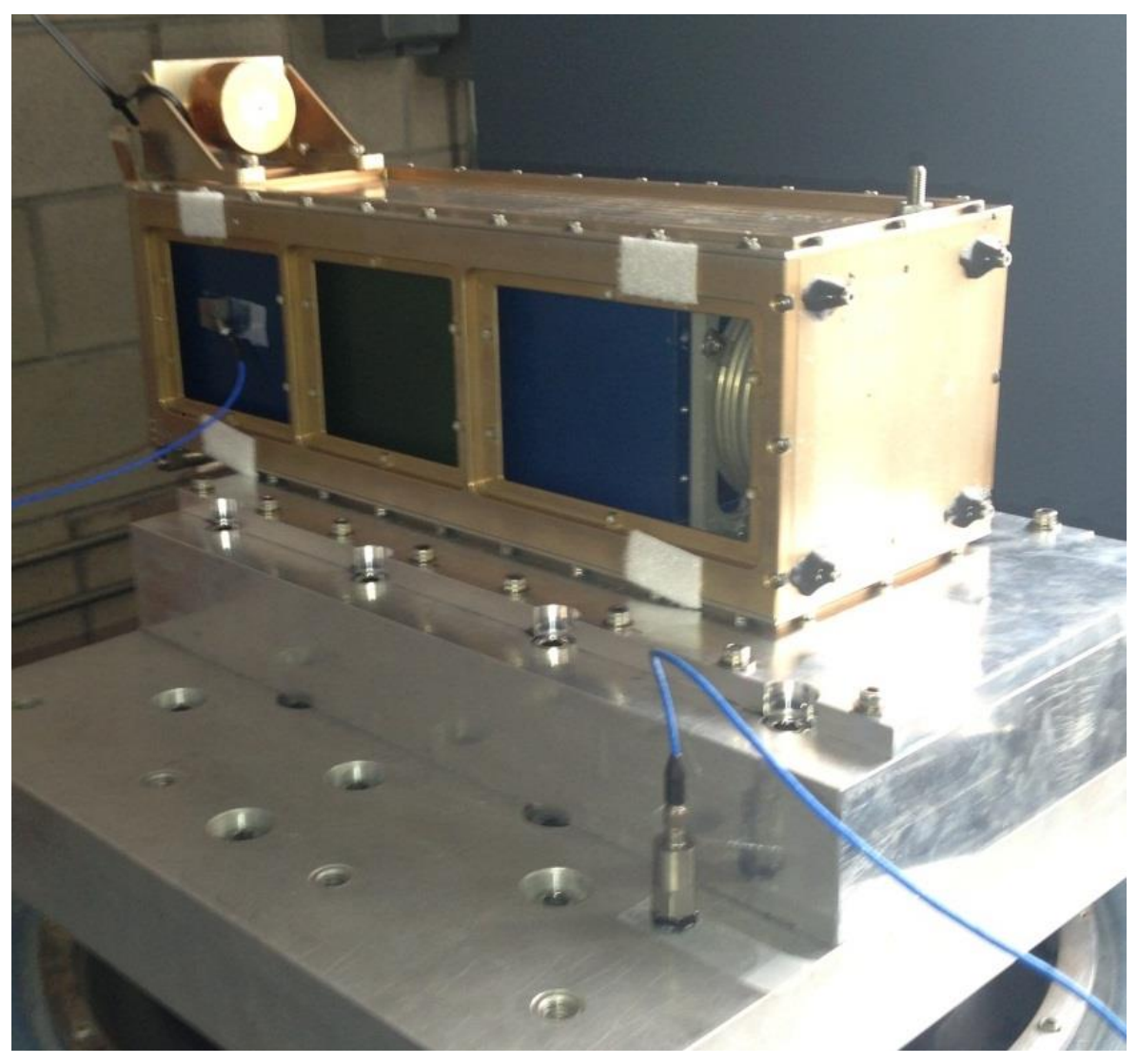

Figure 152: Setup for test numbers 26-2 and 27. 


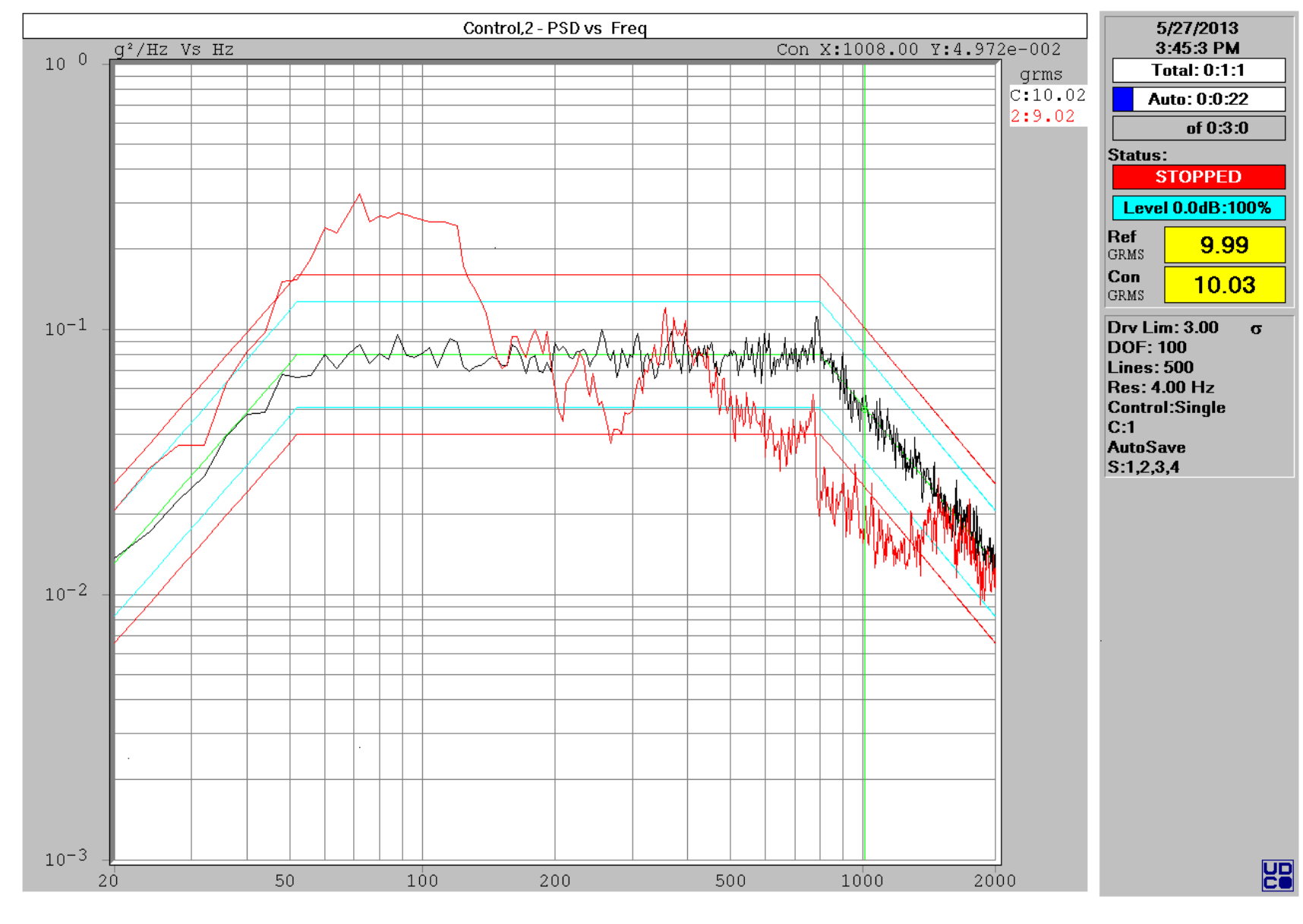

Figure 153: Raw data from test number 26. 

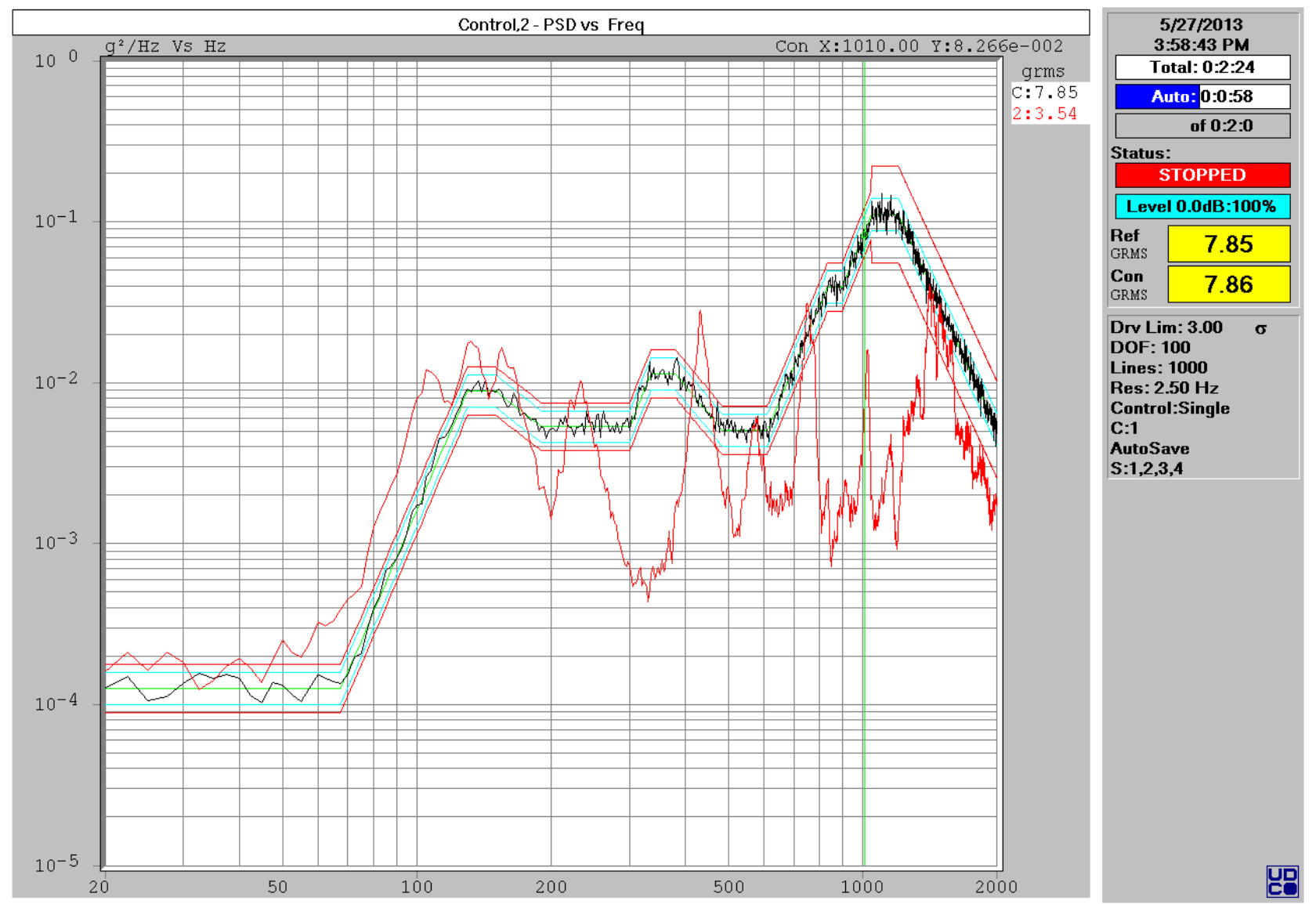

Figure 154: Raw data from test number 27. 


\section{APPENDIX E: Vibration Test Setup and Data from Chapter 6}

This Appendix shows data from the isolator tests that were not shown in the main body of the thesis. 

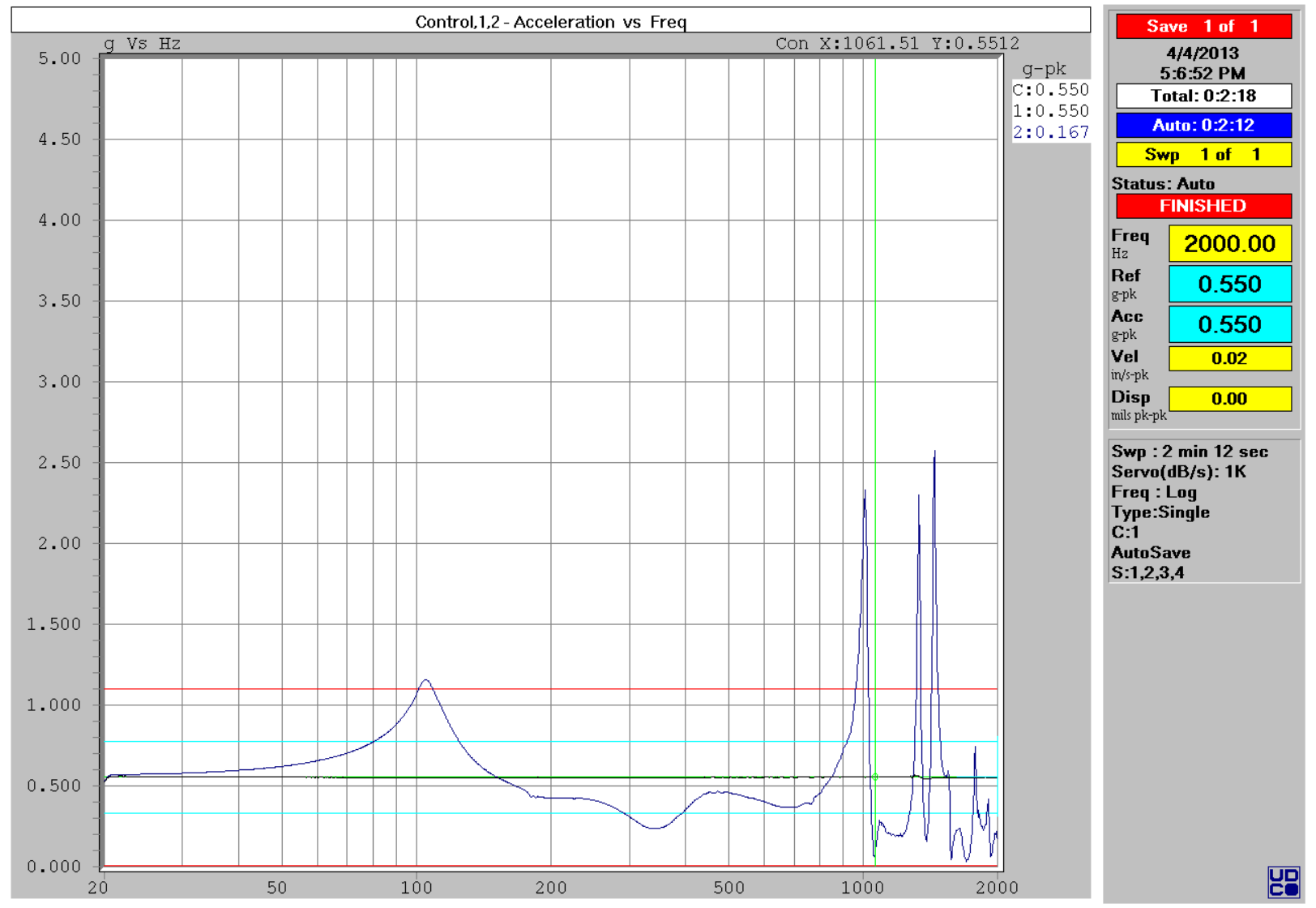

Figure 155: Raw data from $.42 \mathrm{~kg}$ isolated CubeSat $\mathrm{X}$ axis sine sweep. 

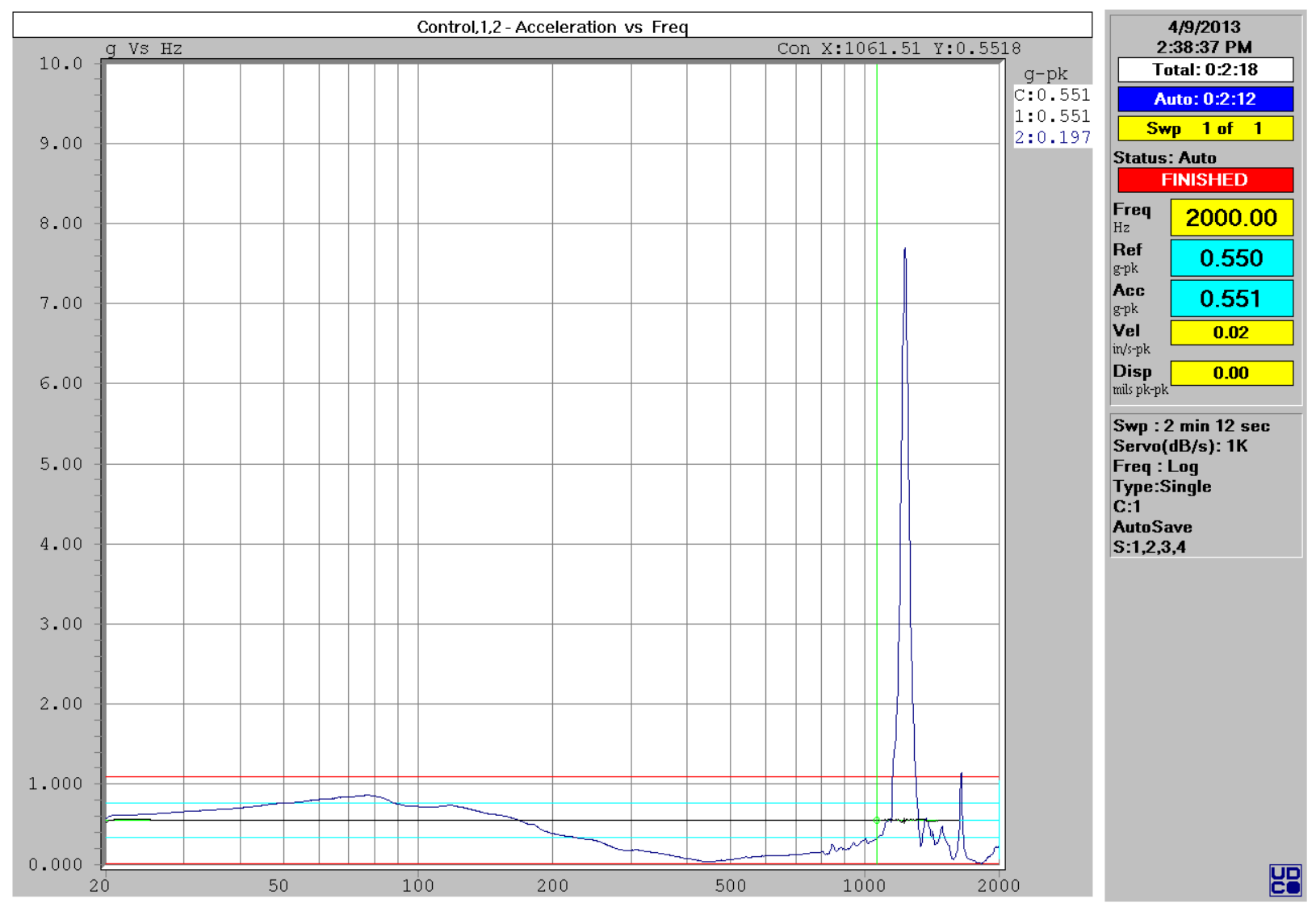

Figure 156: Raw data from .97 kg isolated CubeSat $\mathrm{X}$ axis sine sweep. 

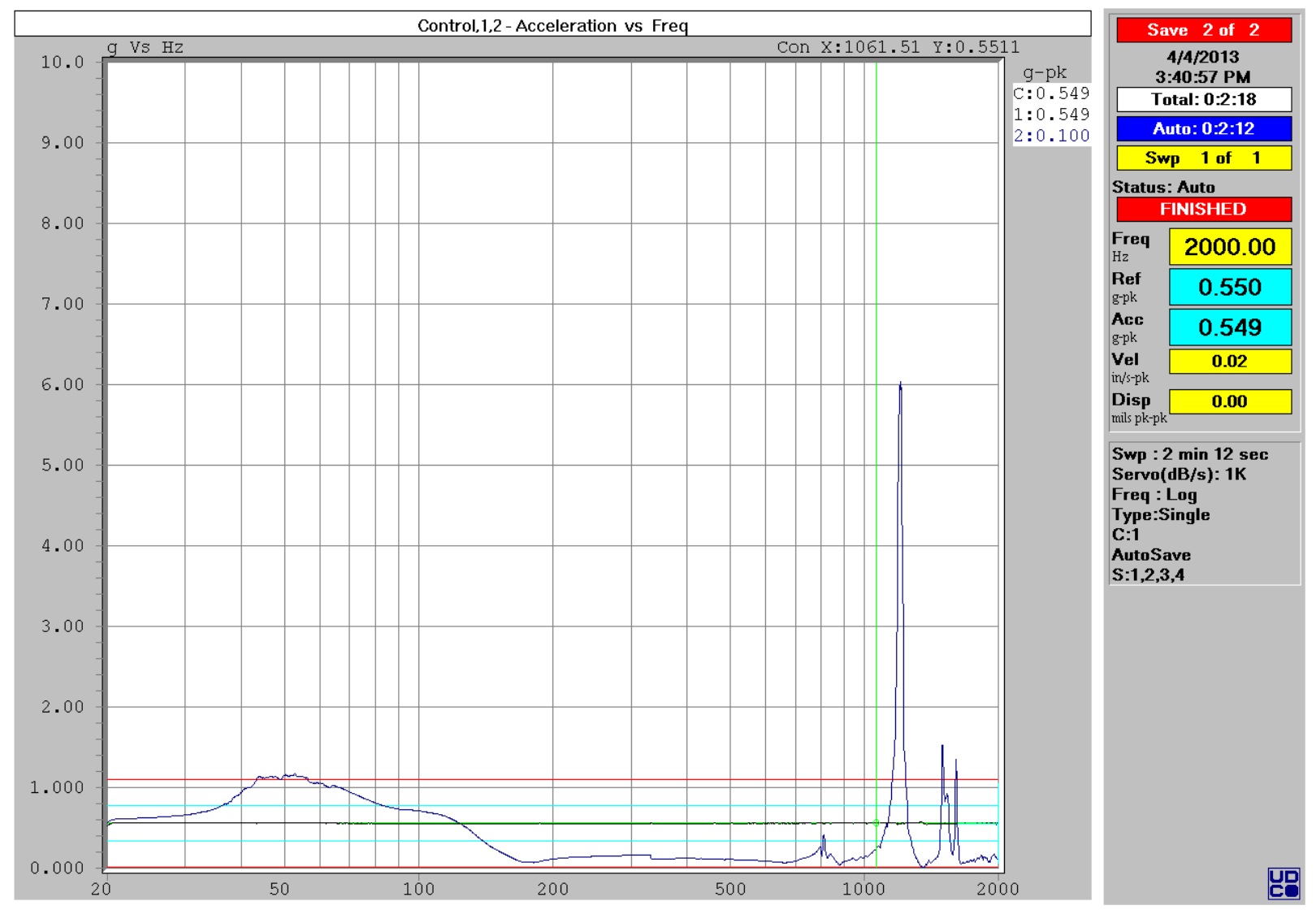

Figure 157: Raw data from $1.33 \mathrm{~kg}$ isolated CubeSat $X$ axis sine sweep. 


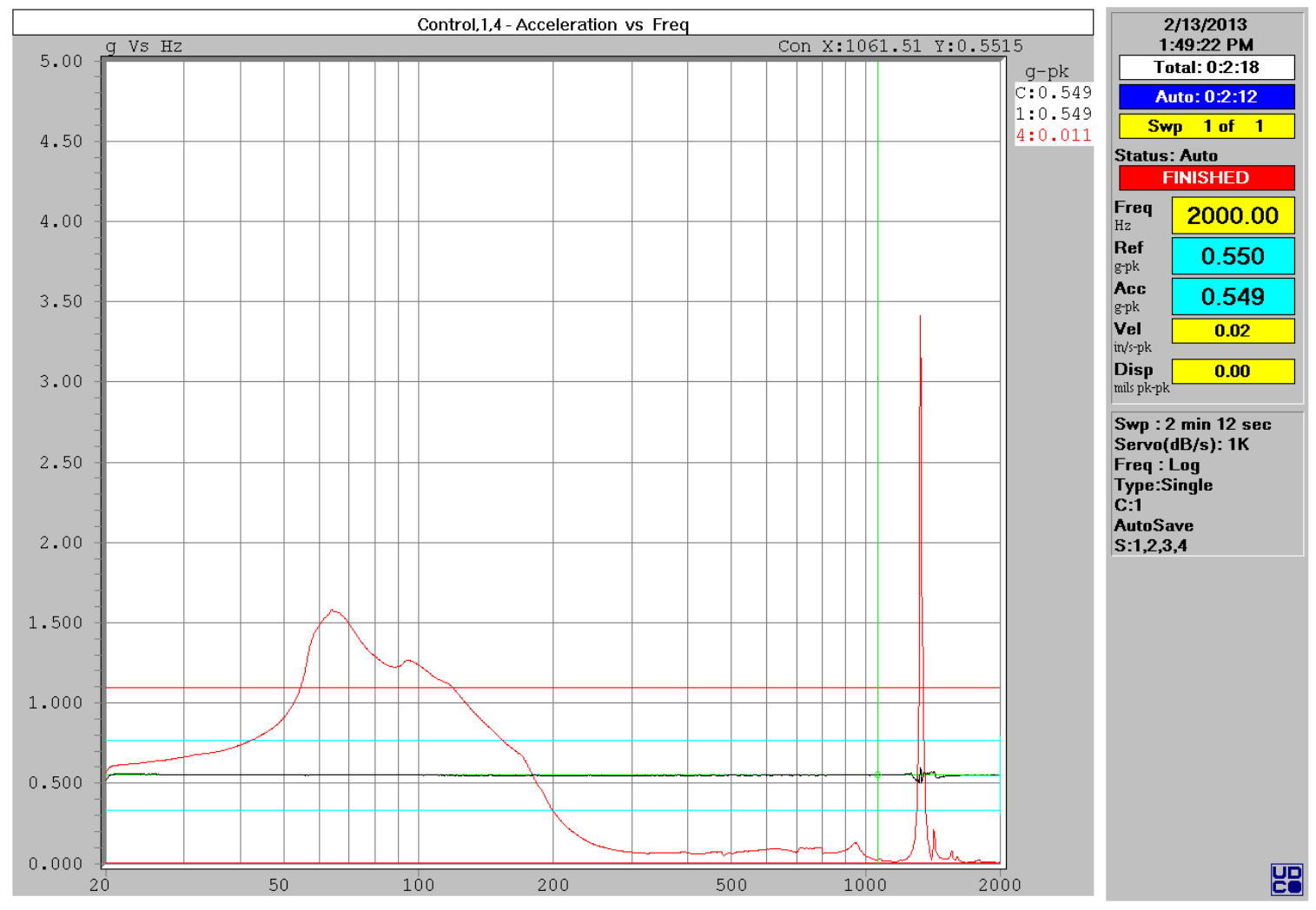

Figure 158: $\mathrm{Z}$ axis sine sweep test of isolated $1.33 \mathrm{~kg}$ Test CubeSat. 
